\title{
Open Source Fault-tolerant Grid Frequency Measurement for Solar Inverters
}

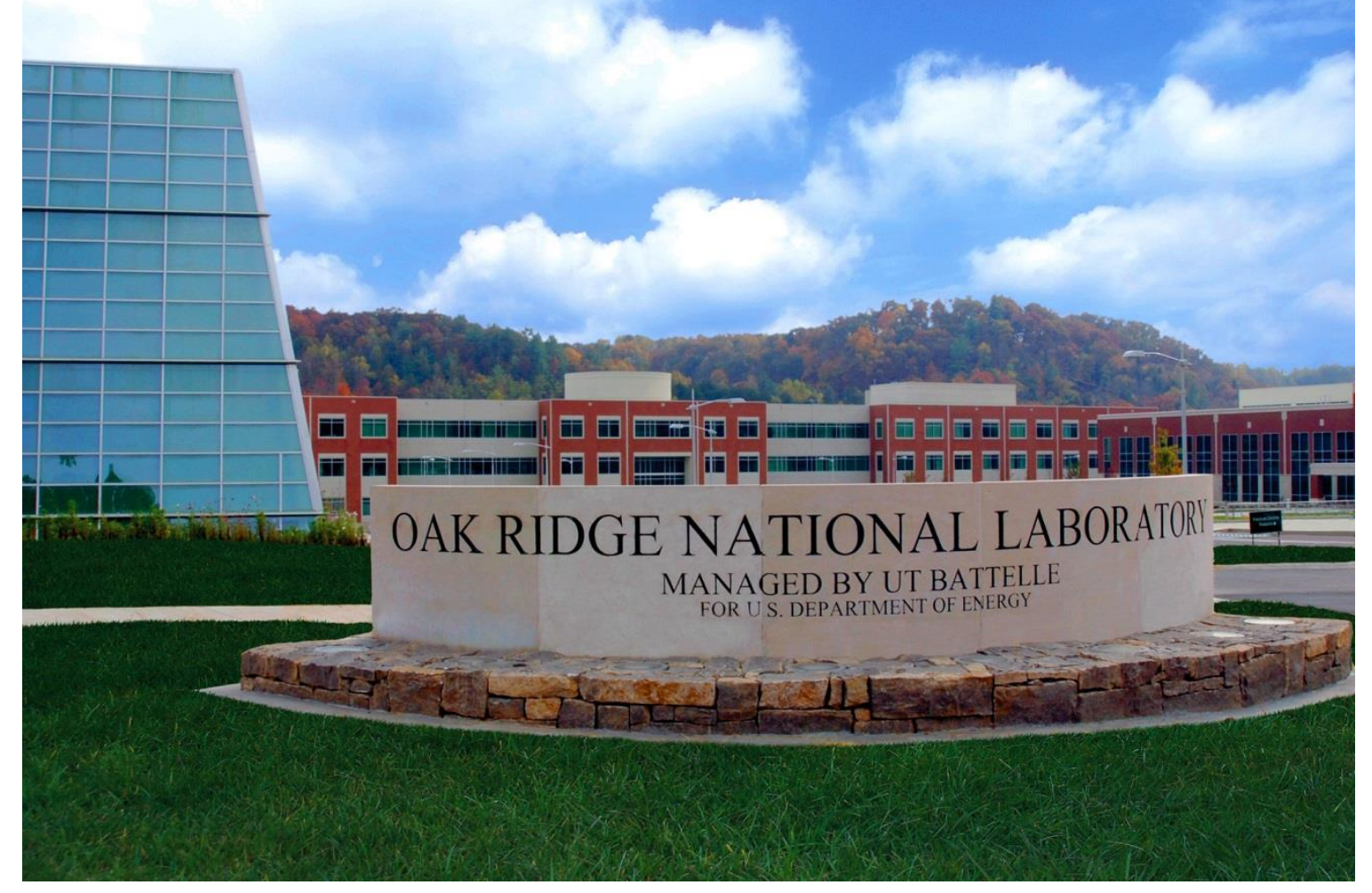

Lingwei Zhan

Bailu Xiao

Fuhua Li

Yilu Liu

September 2021 


\title{
DOCUMENT AVAILABILITY
}

Reports produced after January 1, 1996, are generally available free via US Department of Energy (DOE) SciTech Connect.

Website www.osti.gov

Reports produced before January 1, 1996, may be purchased by members of the public from the following source:

\author{
National Technical Information Service \\ 5285 Port Royal Road \\ Springfield, VA 22161 \\ Telephone 703-605-6000 (1-800-553-6847) \\ TDD 703-487-4639 \\ Fax 703-605-6900 \\ E-mail info@ntis.gov \\ Website http://classic.ntis.gov/
}

Reports are available to DOE employees, DOE contractors, Energy Technology Data Exchange representatives, and International Nuclear Information System representatives from the following source:

Office of Scientific and Technical Information

PO Box 62

Oak Ridge, TN 37831

Telephone 865-576-8401

Fax 865-576-5728

E-mail reports@osti.gov

Website https://www.osti.gov/

This report was prepared as an account of work sponsored by an agency of the United States Government. Neither the United States Government nor any agency thereof, nor any of their employees, makes any warranty, express or implied, or assumes any legal liability or responsibility for the accuracy, completeness, or usefulness of any information, apparatus, product, or process disclosed, or represents that its use would not infringe privately owned rights. Reference herein to any specific commercial product, process, or service by trade name, trademark, manufacturer, or otherwise, does not necessarily constitute or imply its endorsement, recommendation, or favoring by the United States Government or any agency thereof. The views and opinions of authors expressed herein do not necessarily state or reflect those of the United States Government or any agency thereof. 
Electrification and Energy Infrastructures Division

\title{
OPEN SOURCE FAULT-TOLERANT GRID FREQUENCY MEASUREMENT FOR SOLAR INVERTERS
}

\author{
Lingwei Zhan \\ Bailu Xiao \\ Fuhua Li \\ Yilu Liu
}

September 2021

Prepared by

OAK RIDGE NATIONAL LABORATORY

Oak Ridge, TN 37831-6283

managed by

UT-BATTELLE LLC

for the

US DEPARTMENT OF ENERGY

under contract DE-AC05-00OR22725 


\begin{tabular}{|l|l|}
\hline a. Federal Agency & Department of Energy \\
\hline b. Award Number & 34232 \\
\hline c. Project Title & $\begin{array}{l}\text { Open Source Fault-tolerant Grid Frequency } \\
\text { Measurement for Solar Inverters }\end{array}$ \\
\hline & $\begin{array}{l}\text { Lingwei Zhan } \\
\text { R\&D Staff } \\
\text { zhanl@ornl.gov } \\
865-574-7535\end{array}$ \\
\hline d. Principal Investigator & $\begin{array}{l}\text { Dominic Lee } \\
\text { Program Manager } \\
\text { leedf@ornl.gov } \\
865-241-0775\end{array}$ \\
\hline e. Business Contact & $09 / 22 / 2021$ \\
\hline f. Submission Date & N/A \\
\hline g. DUNS Number & ORNL \\
\hline h. Recipient Organization & Start: 10/1/2018 \\
\hline i. Project Period & \multicolumn{2}{|l}{} \\
\hline j. Submitting Official Signature $09 / 30 / 2019$ \\
\hline
\end{tabular}




\section{CONTENTS}

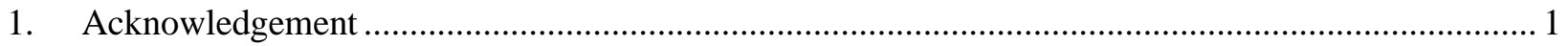

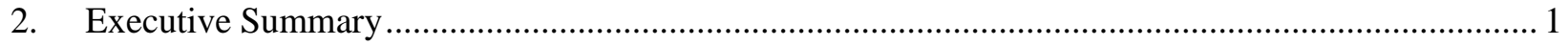

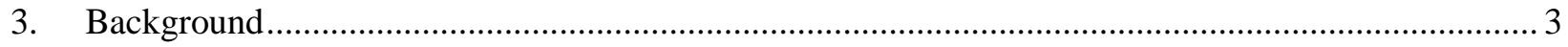

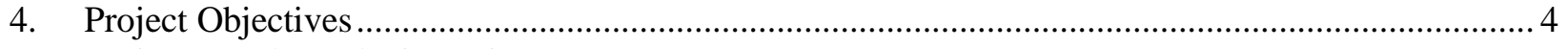

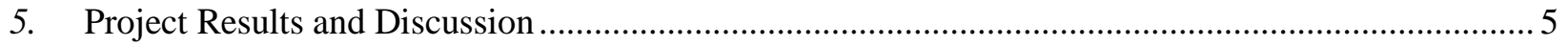

5.1 Fault-tolerant Grid Measurement Algorithm .................................................................. 5

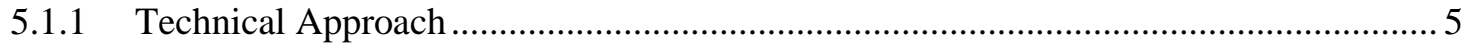

5.1.2 Development of a Transient Detector .................................................................... 7

5.1.3 Development of an adaptive frequency estimation algorithm ................................... 16

5.1.4 Accuracy and reliability evaluation, and further optimization of the developed

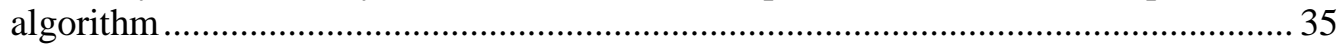

5.2 Computation Complexity Analysis and Computation Cost Test .......................................... 59

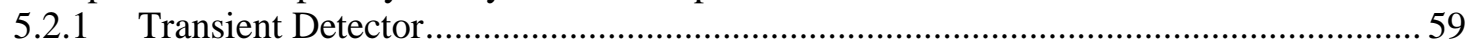

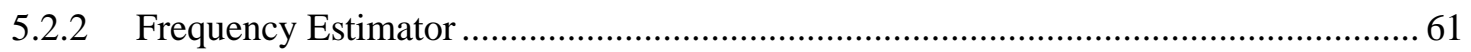

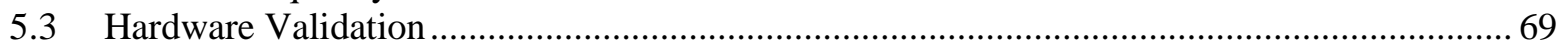

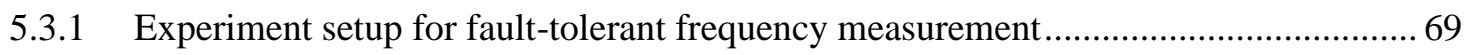

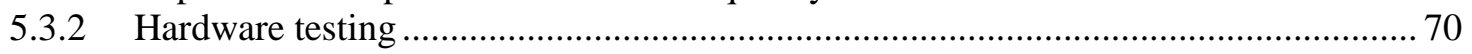

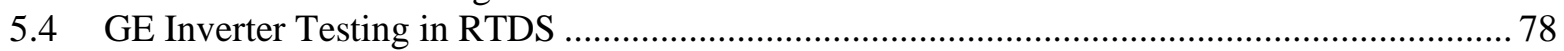

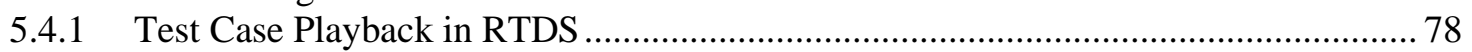

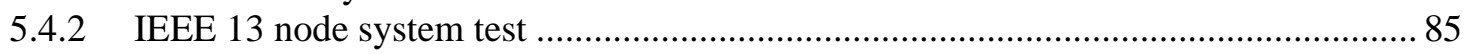

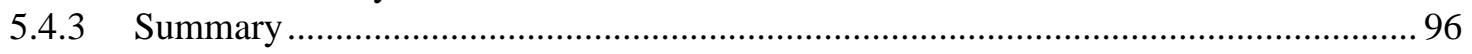

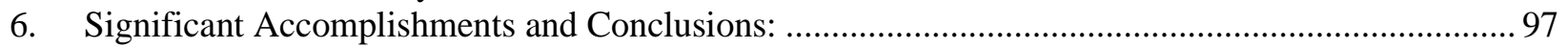

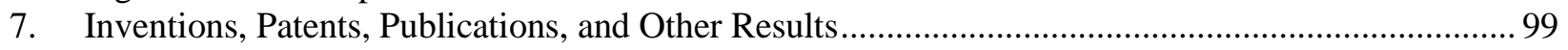

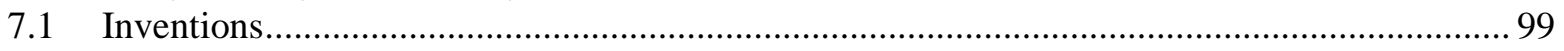

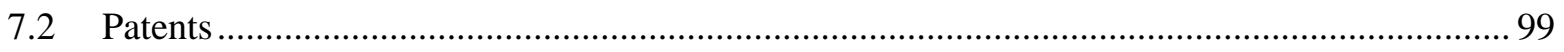

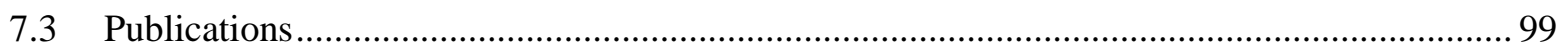

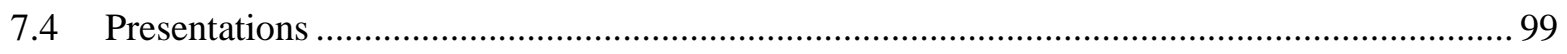

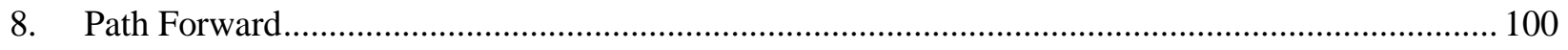

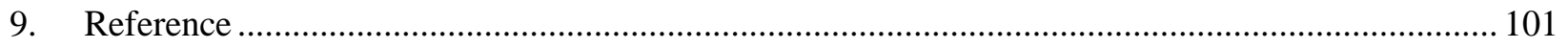

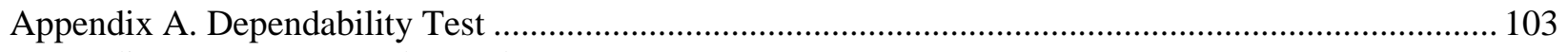

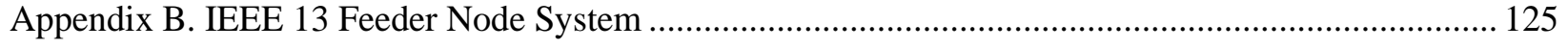




\section{ACKNOWLEDGEMENT}

This material is based upon work supported by the U.S. Department of Energy's Office of Energy Efficiency and Renewable Energy (EERE) under the DOE SETO National Laboratory Call for Proposals Award Number 34232.

\section{EXECUTIVE SUMMARY}

Many critical electric grid operations rely on accurate system frequency measurement [1], however, the frequency measurement can be easily undermined by frequent transient events. On August 16, 2016, a false frequency measurement of below $57 \mathrm{~Hz}$ during a series of system faults led to the tripping of 700 MW solar inverters instantaneously in Southern California, United States [2]. Later, it was found out that the actual system frequency only dropped to about $59.867 \mathrm{~Hz}$, and the frequency measurement algorithm used in solar inverters failed to provide the accurate measurements [2]. In addition, hundreds of such events at lower magnitude have happened based on records from NERC. After this event, recommend by NERC [2], inverter manufactures and affected utilities developed a temporary correction plan by implementing a five (or two) -second time delay for low (or high) frequency protection to avoid the potential false trips caused by incorrect frequency measurements [2]. However, this approach is far from perfect because the added time delay will prevent solar inverters from taking protective actions if system frequency does deviate from nominal frequency significantly.

The event revealed that the problem is that the frequency measurement during system transient faults could have large errors, which could cause the operation of protection system mistakenly. To solve the problem, we first investigated and tested existing measurement technologies, and understand the technical challenges of grid measurements during system transients. Based on investigation and test, we developed the fault-tolerant measurement technology that will overcome the measurement problems of existing measurement methods, thus providing reliable measurements for grid controls and protections. The developed technology has been optimized and evaluated under different types of steady-state and transient conditions. Further, the developed measurement method has been implemented on low-cost hardware to verify it has low computation cost for easy system integration. The key advantage of the developed measurement technology is that it can provide reliable measurement during system transients, and it not only contributes to a highly secure protection system, but also contributes to many other grid applications that rely on reliable measurements such as frequency regulation, smooth DER integration, voltage regulation, stability predication, fast load shedding. Additionally, because the grid measurement is a fundamental component for many devices/systems (e.g., Relays, PMUs, wind turbine, energy storage, etc.), the success of this project will have a broad impact on these areas, ultimately contributing to a more reliable and secure bulk system.

Some key features and performances of the new technology have been summarized as follows.

- System transient faults, particularly phase angle step change that can cause large measurement errors, could be detected within a quarter fundamental frequency cycle.

- The maximum frequency measurement errors in two cycles following a transient fault with phase angle change are within $0.05 \mathrm{~Hz}$, and the errors are within $0.005 \mathrm{~Hz}$ six cycles (i.e., 0.1 second) following the event.

- The transient detector has extremely low computational cost and detects system anomalies sample by sample with high resolution and high speed. The lightweight detector enables its easy implementation into hardware without introducing much additional hardware burden. 
- Working with the transient detector, the adaptive frequency estimator can minimize frequency measurement errors during system transients, thus providing reliable and accurate measurements during transients.

- From the system point of view, the fault-tolerant measurements provided by the developed measurement technology can enhance the protection reliability and security during system transient events. In addition, thanks to the robust and accurate measurements, the performances of the grid services participated by DERs could be also improved.

To promote the developed technology for further development and industry adoption, the developed technology has been published in the paper "Fault-tolerant grid frequency measurement algorithm during transients", and "Recent Development of Frequency Estimation Methods for Future Smart Grid", which are all published in open access journals. The project outcomes were also presented in 2019 IEEE PES general meeting and several times in North American SynchroPhasor Initiative (NASPI) conference. A company plans to utilize our technology to develop a new grid monitor. 


\section{BACKGROUND}

The Discrete Fourier transform (DFT) based measurement algorithms are one of the most common measurement algorithms for grid parameter estimation such as rms, phase angle, frequency [3]. Over the past few years, many DFT based algorithms have been developed to enhance its measurement accuracy under steady-state and/or dynamic grid conditions [4]-[17]. For example, an adaptive band-pass filter utilizing exponential modulation filter has been proposed to reduce measurement errors at the presence of large frequency deviations [6],[7]. Measurement accuracy of different algorithms including FIR filter, extended Kalman filtering (EKF), and enhanced DFT method have been compared in detail under different grid conditions [13]. Two artificial signals that have 90-degree phase difference were constructed by the Clarke transformation to address the frequency spectrum leakage of DFT [16]. A multi-module approach was developed to enhance both steady-state and dynamic measurement accuracies [17], in which each module was developed to eliminate some specific errors. Besides DFT-based measurement algorithms, some signal model-based algorithms have been developed to further improve the accuracy under dynamic conditions [18]-[21]. However, a key drawback of the state-of-the-art algorithms is that they cannot perform measurements accurately during system transient faults. In the Blue Cut Fire event, there was a phase angle jump of about 26 degrees in the voltage waveform during the transient fault. The phase angle jump fault will cause waveform discontinuity, and these algorithms will fail to provide reliable measurements during this period because they typically assume the waveform to be measured is continuous, no matter what method (DFT, PLL, EKF, FIR, or Taylor WLS) is used for estimation.

In fact, the measurement errors during the system transient faults like phase-jump is not required in the IEEE Standard [22]. As a result, although a measurement instrument can pass the strict IEEE Standard, it could still be the source of the problem in the future if we have similar system transient faults, which could happen again. Therefore, developing the fault-tolerant measurement technology is the key to solve the problem. 


\section{PROJECT OBJECTIVES}

For existing measurement technologies, they lack the capability to "perceive" transient faults, which leads to significant measurement errors during transients. The overall objective of this project is to develop a fault-tolerant frequency measurement algorithm, as illustrated in Figure 1, thus providing a fundamental solution to frequency dependent grid control and protection. Overall, we proposed a two-step approach (a transient fault detector followed by an adaptive frequency estimator) for fault-tolerant frequency estimation. To be specific, we developed a real-time "transient detector" to detect the waveform discontinuities such as phase jumps and magnitude step change, which are responsible for significant frequency errors. With the help of successful discontinuities detection, then we developed an adaptive and intelligent frequency estimator. According to the transient detection information provided by the transient detector, the frequency estimator can adjust the estimation window to minimize the estimation errors during system transients. The complete measurement method consisting of transient detector and intelligent frequency estimator were tested and optimized by synthetic signals and transient fault signals from IEEE benchmark system models to evaluate the measurement performance in terms of accuracy, dependability, and security. Further, the developed measurement technology was implemented on lowcost hardware and the real-time measurements were achieved. It demonstrated the low computation cost of the method, which is critical for easily and widely industry adoption.
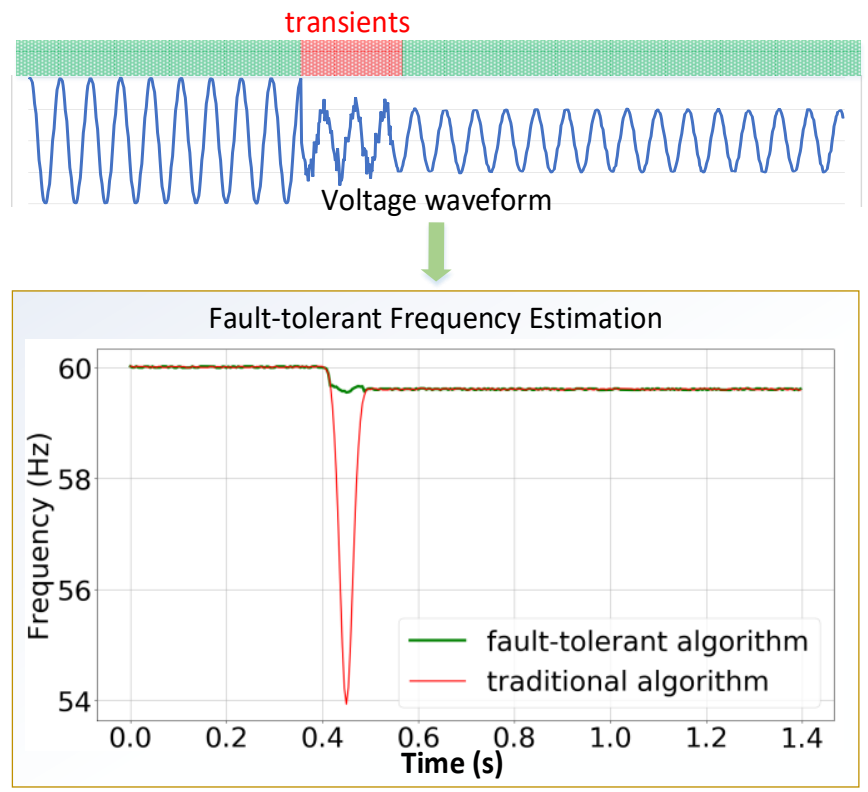

Figure 1 Illustration of fault-tolerant frequency measurement 


\section{PROJECT RESULTS AND DISCUSSION}

\subsection{FAULT-TOLERANT GRID MEASUREMENT ALGORITHM}

\subsubsection{Technical Approach}

Existing measurement algorithms suffer from significant measurement errors since the measurement model of these algorithms assume the measured grid signals are in the form of cosine waveform as follows:

$$
x(t)=\sqrt{2} A_{0} \cos (2 \pi f t+\varphi)(1)
$$

where $\mathrm{A}_{0}$ is the magnitude of the of power grid signal, $f$ is the fundamental frequency of the power grid signal, and $\varphi$ is the phase angle of the power grid signal.

For existing grid measurement algorithms, these parameters are assumed to be constant or slow-varying variables, so the measurement errors are low when the assumptions are true. Unfortunately, during power grid system transient events, these parameters can experience significant and sudden change, such as phase angle or magnitude step change, which can introduce substantial errors to measurement results. For example, during a phase angle step change, the grid signal $x(t)$ will exhibit discontinuity feature. Figure 2 shows the point-on-wave of the grid signal with a 90-degree phase angle step change. We could clearly see the discontinuity of the signal due to the phase angle step change.

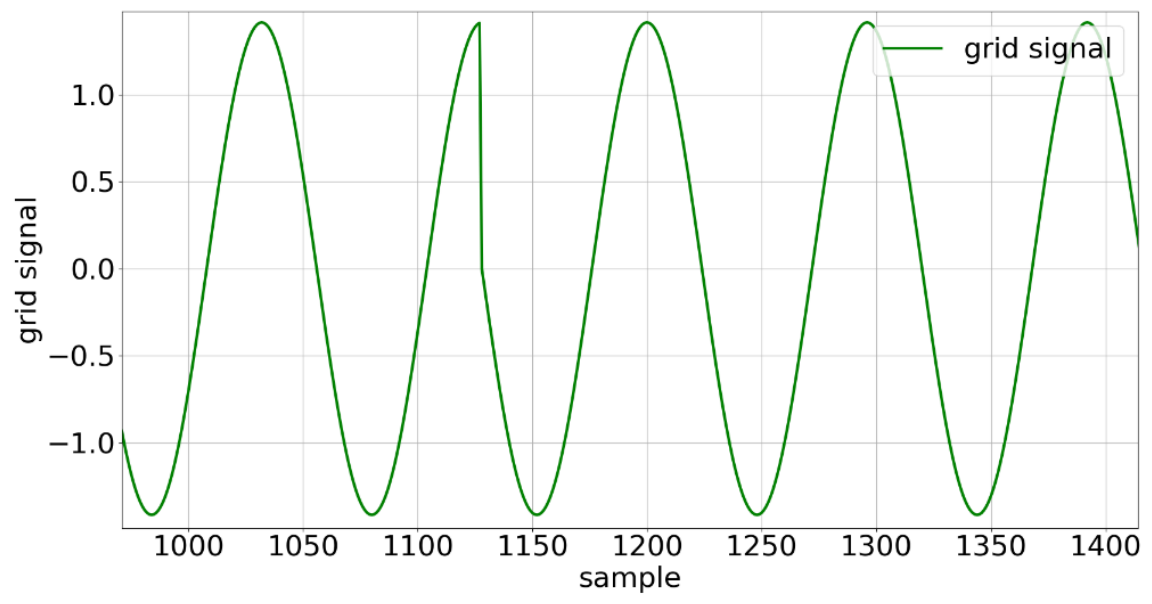

Figure 2 point on wave data with 90-degree step change

The discontinuity of signals will introduce significant measurement errors since commonly used algorithms assume the measured grid signals are slow varying steady signals. Figure 3 and Figure 4 show the frequency measurements of a DFT based and a Phase-locked loop (PLL) based measurement algorithm, respectively. 


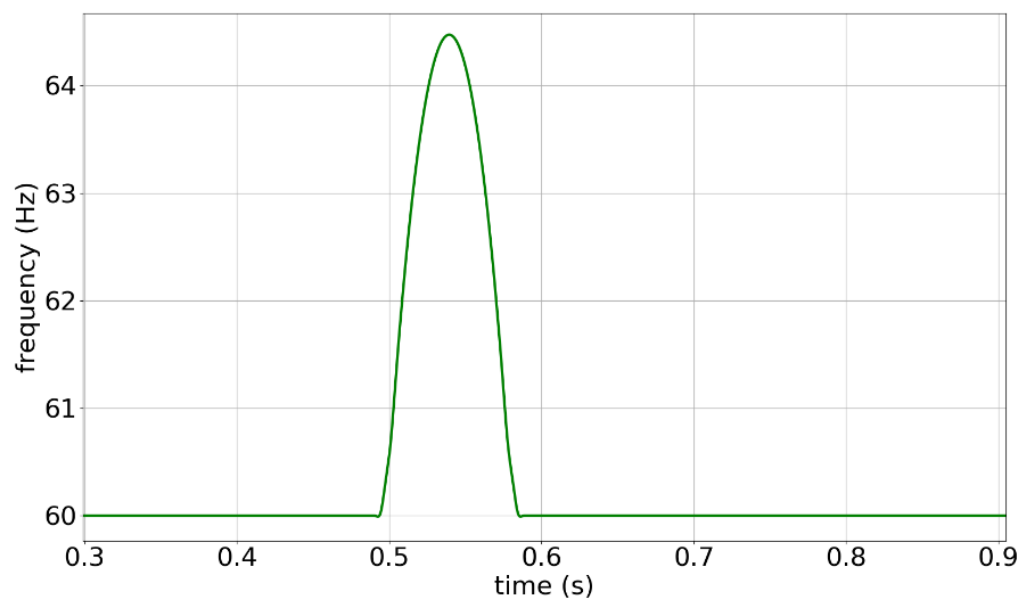

Figure 3 Frequency measurements of DFT based frequency estimation algorithm

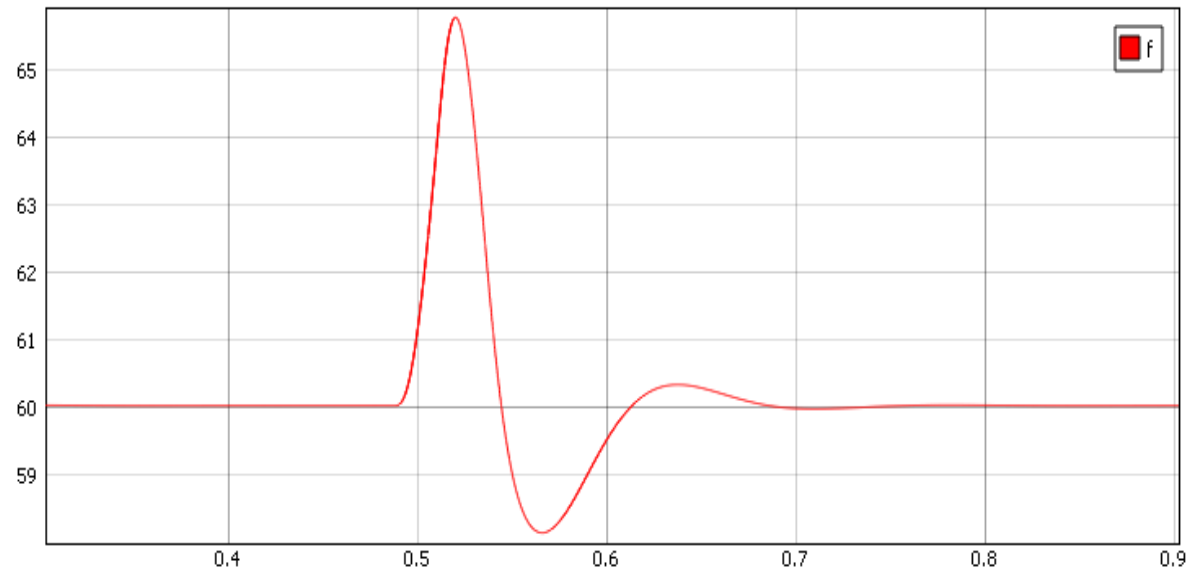

Figure 4 Frequency measurements of PLL based frequency estimation algorithm

It can be observed that when the grid signals have a step change, the measurement algorithms will have large measurement errors.

The existing measurement algorithms could not tolerate such signal distortions because they cannot "see" the distortions of the grid signals, therefore they cannot adapt to the change of grid signals. In this project, we propose a two-step approach for fault-tolerant frequency measurements. In the first step, we develop a grid transient detector, and it will be able to detect the transients of grid signals, particularly the step change of the grid signals. The second step is the adaptive frequency estimator, and it will be able to adapt to the grid transient events based on the detection results in the first step. The overall concept of this approach compared to the traditional algorithms are shown in Figure 5. 


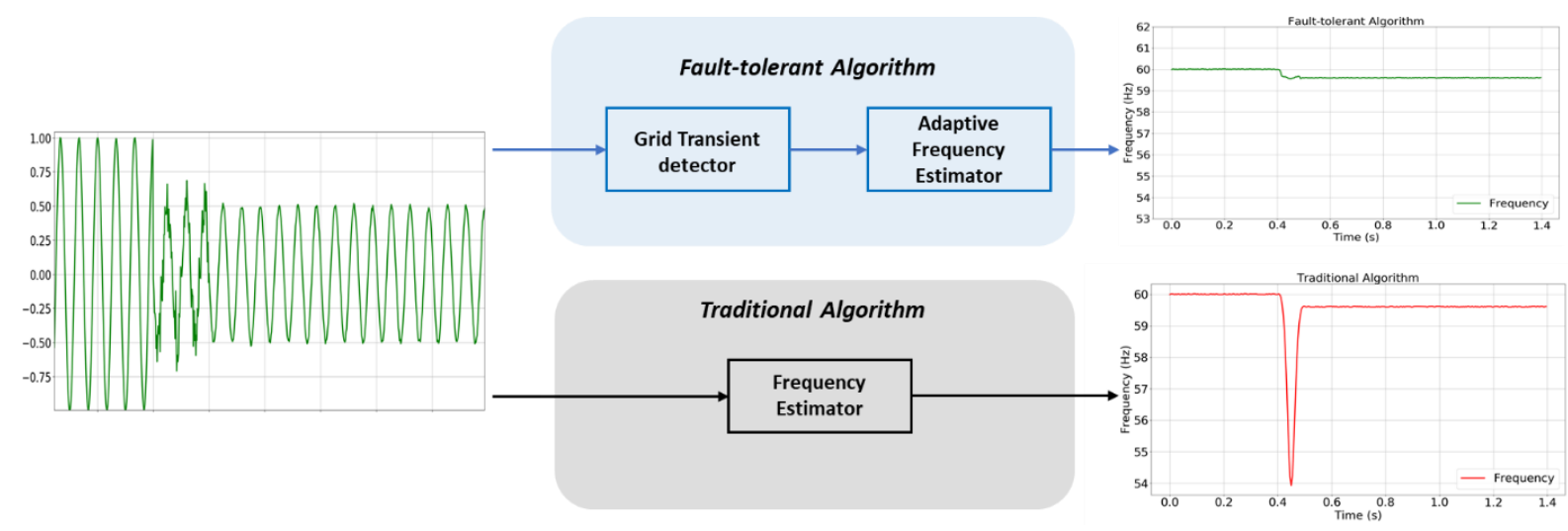

Figure 5 Architecture of the proposed fault-tolerant algorithm vs traditional algorithm

Therefore, the key and first step of the project is to develop a transient fault detector.

\subsubsection{Development of a Transient Detector}

When the power grid operates normally without any transient event, the grid signals can be approximately represented by the following equation:

$$
x(t)=\sqrt{2} A_{0} \cos (2 \pi f t+\varphi)(2)
$$

As the power grid signal is periodic, we will have the following equation,

where $T$ is the period of the grid signal.

$$
x(t)-x(t-T)=0(3)
$$

When the grid signals experience transient events, the signals will be distorted, and the periodicity will not maintain. As a result, $x(t)-x(t-T)$ will not be equal to 0 . Instead, $x(t)-x(t-T)=\alpha$. The larger of the absolute value of $\alpha$ (i.e., $|\alpha|)$, the larger deviation of the grid signal from the normal periodic signal.

Based on this observation, the proposed fault detector is represented as follows:

$$
\gamma(t)=\left|\int_{t=0}^{T}[x(t)-x(t-T)] d t\right|(4)
$$

It is the integration of $x(t)-x(t-T)$ over one fundamental frequency cycle of grid signals. For steadystate grid signals, $x(t-T)$ is close to 0 , therefore $\gamma(t)$ will be close to 0 .

In practice, the grid signals are digitally sampled. Assuming there are $\mathrm{N}$ samples in one fundamental frequency cycle. Then the detector equation could be represented as follows,

$$
\gamma(k)=\left|\sum_{k=0}^{N} x(k)-x(k-N)\right|(5)
$$

Figure 6 shows a simulation of the detector value $\gamma$ (Figure 6(b)) when the measured signals are ideal sinusoidal (Figure 6(a)). It verified that ideally the detector value $\gamma$ is equal to 0 , as expected in previous analysis. 


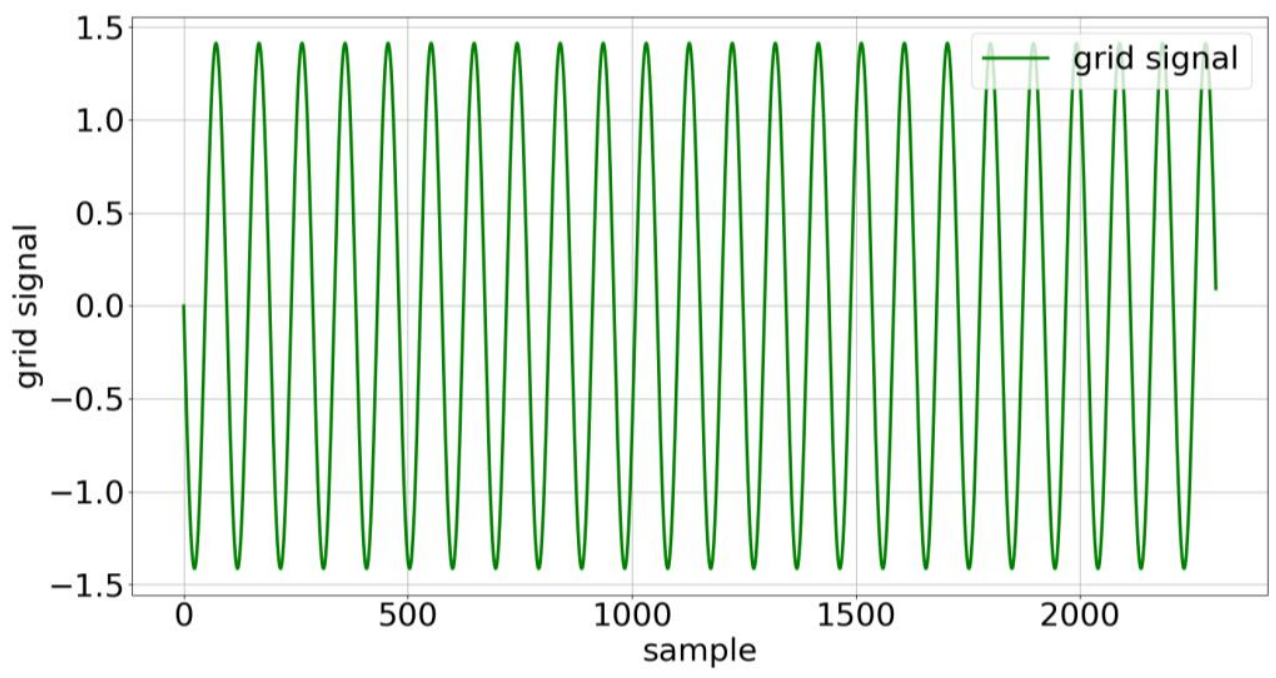

(a). grid signal

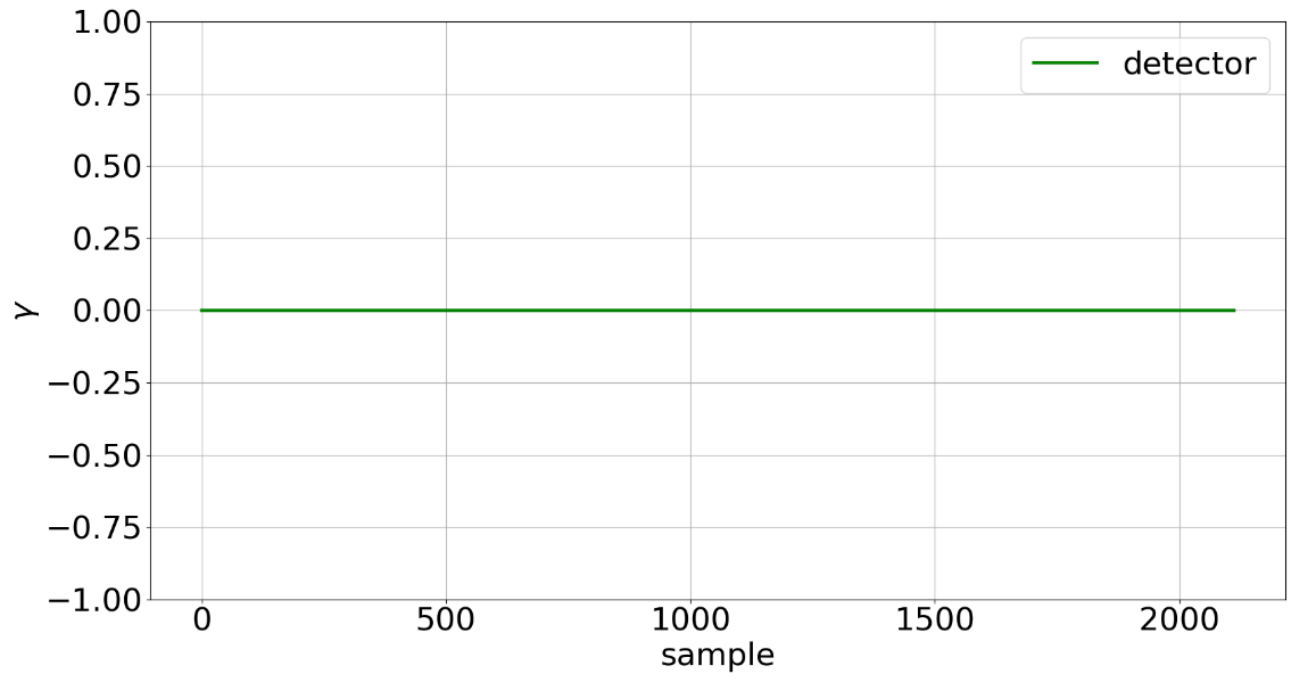

(b). fault detector output

Figure 6 detector output - ideal grid signal

The detector has been implemented using Python/C++ for performance testing. Consider the grid signals are usually distorted by noise and harmonics. Therefore, the performance of the detector needs to be evaluated under these conditions.

\subsubsection{Noise Test}

According to our previous research [17], [23], the noise level of grid signals is about $60-70 \mathrm{~dB}$. Please note that although we have developed linear averaging filter to minimize the noise in previous work [17], [23], which could significantly reduce the noise. In this test, we still use high noise level for a more conservative testing. The impact of noise at different levels from $50 \mathrm{~dB}$ to $80 \mathrm{~dB}$ on detector has been evaluated and the results are shown in Figure 7 and summarized in Table I.

We can see that the impact of noise on the detector is weak. 
Table I Maximum fault detector output under different levels of white noise

\begin{tabular}{|l|l|l|l|l|}
\hline Noise level $(\mathrm{dB})$ & $50 \mathrm{~dB}$ & $60 \mathrm{~dB}$ & $70 \mathrm{~dB}$ & $80 \mathrm{~dB}$ \\
\hline Fault detector output $(\gamma)$ & 0.25 & 0.1 & 0.025 & 0.0125 \\
\hline
\end{tabular}

The robustness of the detector to the noise is crucial for reliability of the fault detector because we need avoid falsely triggering the fault detector due to the noise.

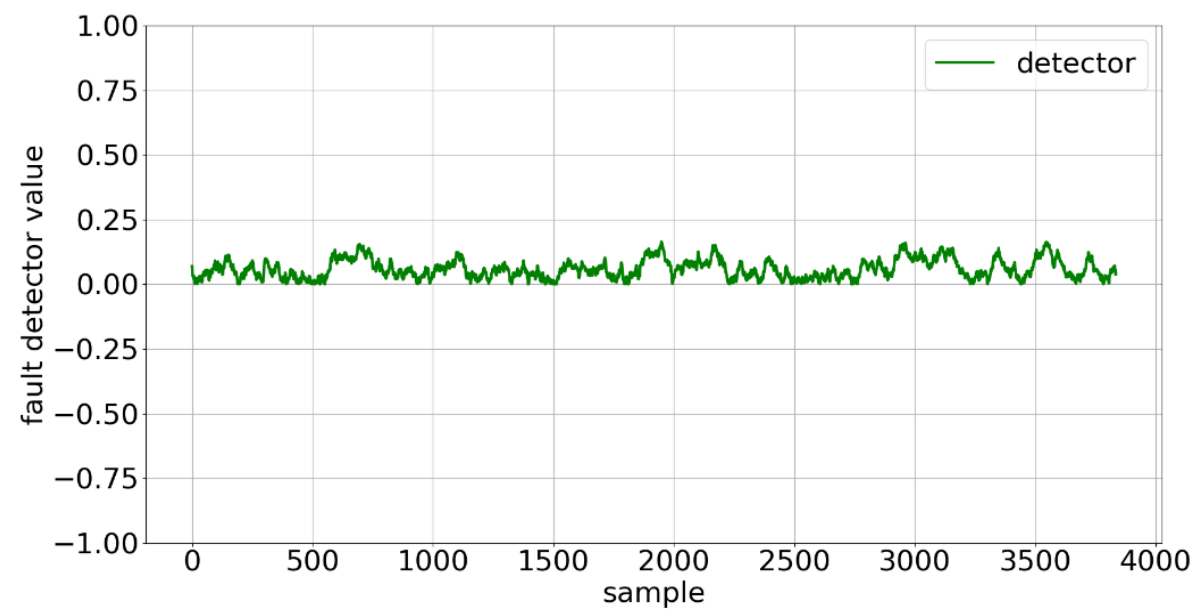

(a). detector output at $50 \mathrm{~dB}$ noise

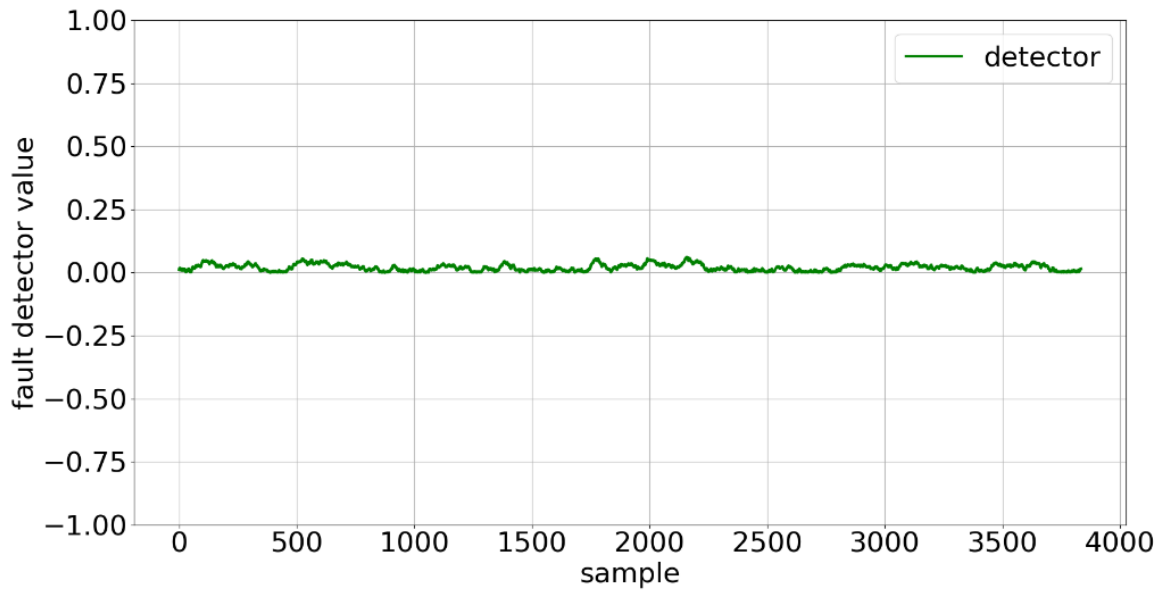

(b). detector output at $60 \mathrm{~dB}$ noise 


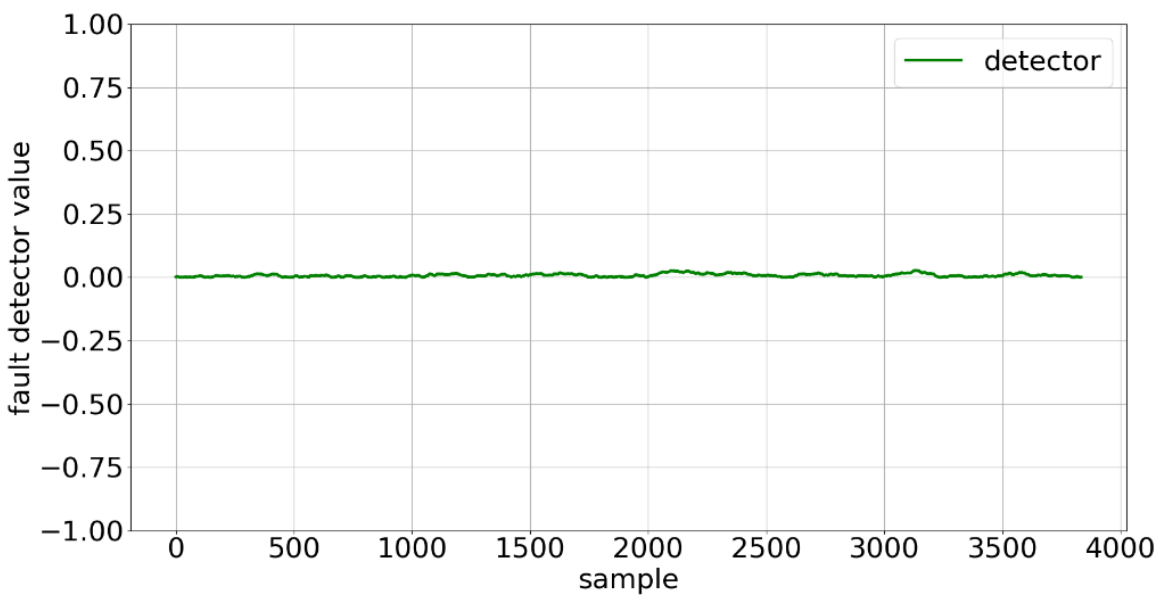

(c). detector output at $70 \mathrm{~dB}$ noise

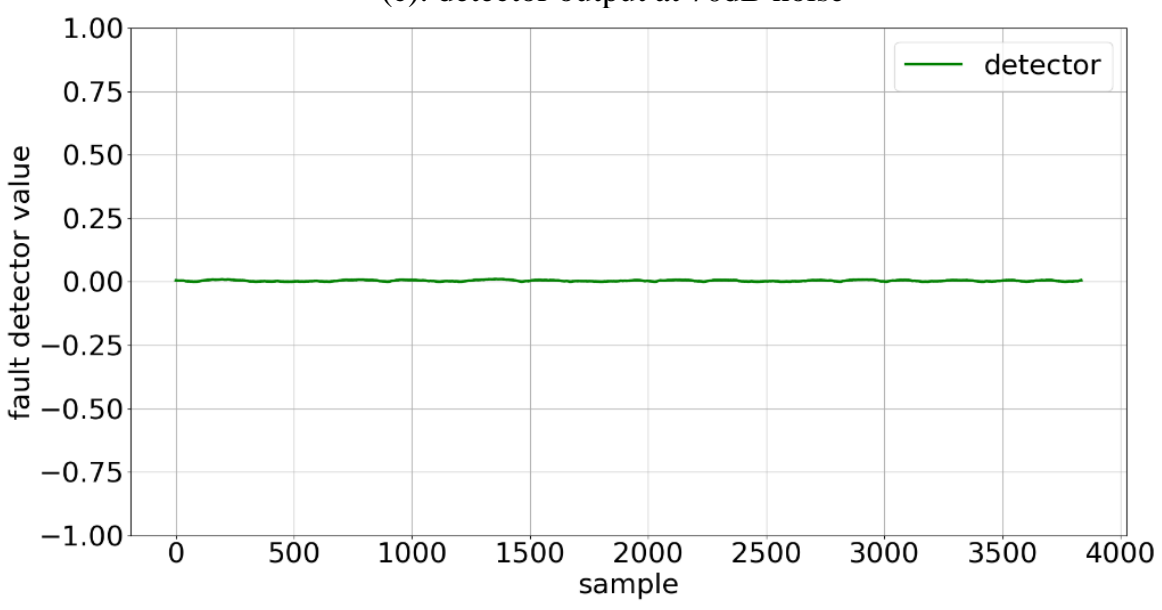

(d). detector output at $80 \mathrm{~dB}$ noise

Figure 7 detector output - grid signal with different levels of noise 


\subsubsection{Harmonics test}

The power grid signals could also be distorted by harmonics. $10 \%$ of harmonics is used for testing, which is much higher than the typical harmonic magnitude in actual environments. It should be also noted that in practice harmonics will be filtered by low-pass filter (analog or digital) to minimize its impact on grid measurements. The higher of the order of the harmonics, the lower its magnitude thanks to the low-pass filter. The raw point-on-wave grid waveforms with harmonics are also shown in the following figures (Figure 8 - Figure 11) for better understanding.
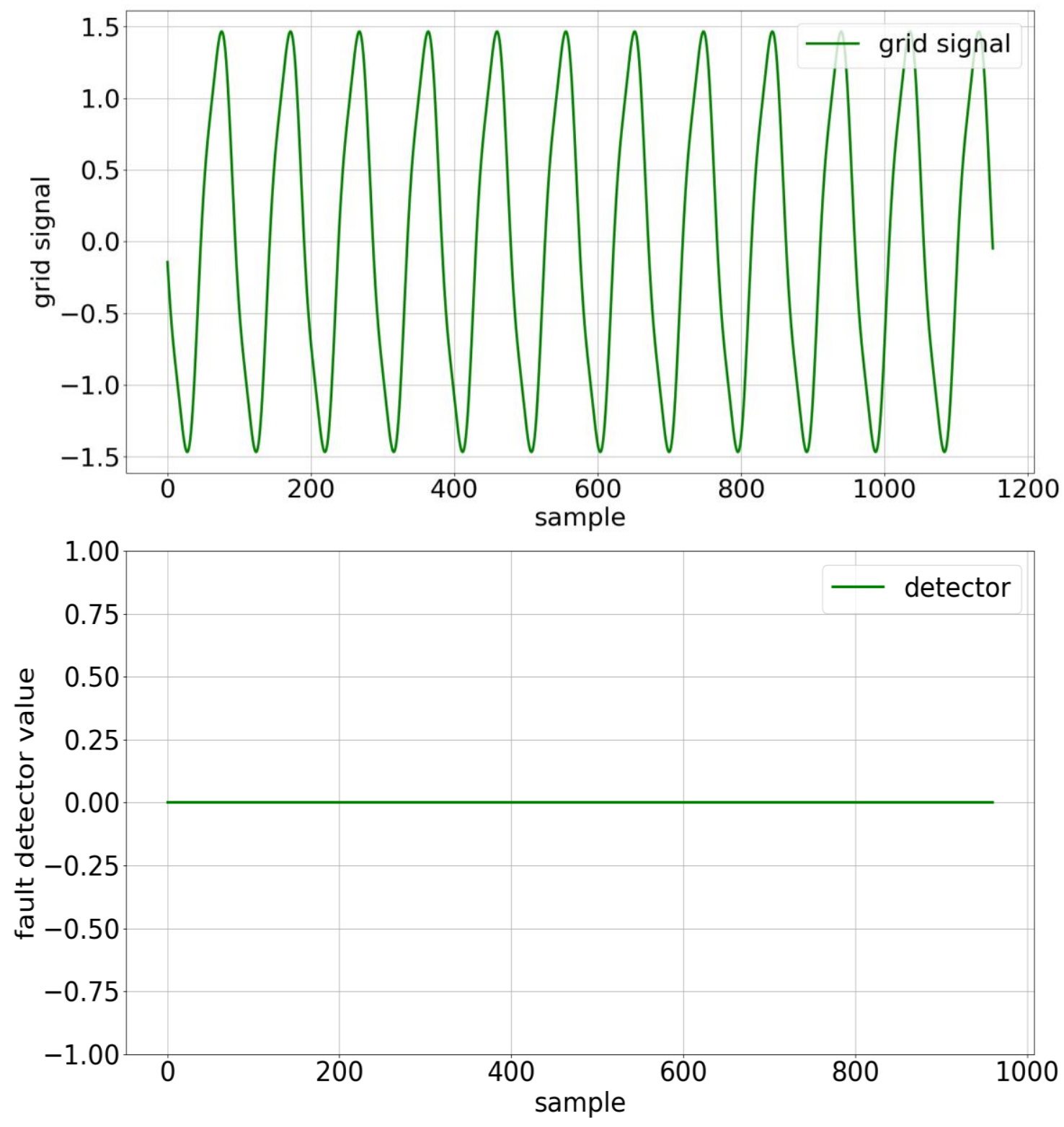

Figure 8 detector output - grid signal with $10 \% 3^{\text {rd }}$ harmonic 
It could be observed that although the grid signals are highly distorted by harmonics, the impact of harmonics on fault detector is low. This is also very important since usually frequency estimation algorithms can tolerate harmonics, so it is desired that the signal distortions caused by harmonics do not impact the fault detector.
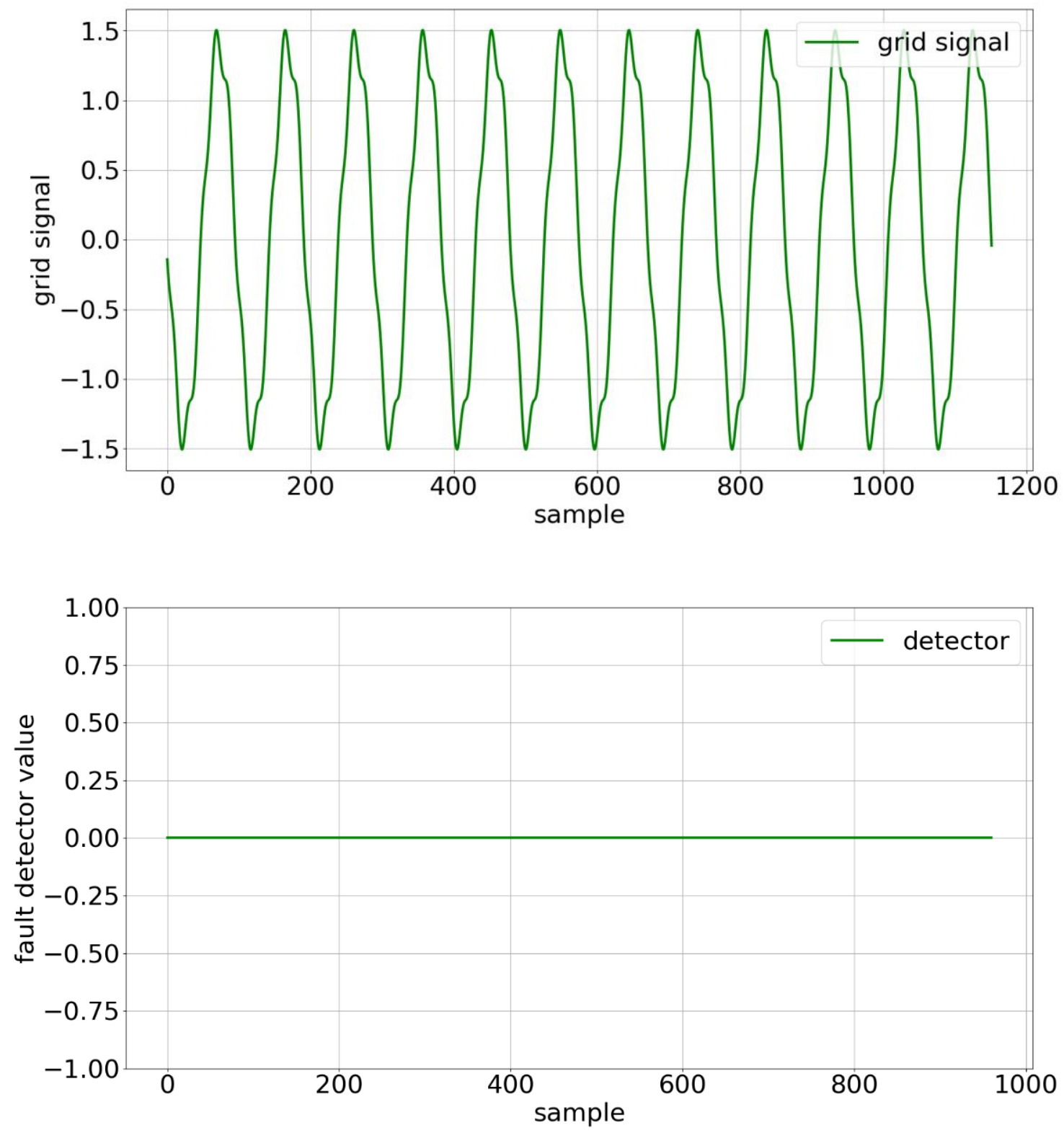

Figure 9 detector output - grid signal with $10 \%$ 5th harmonic 

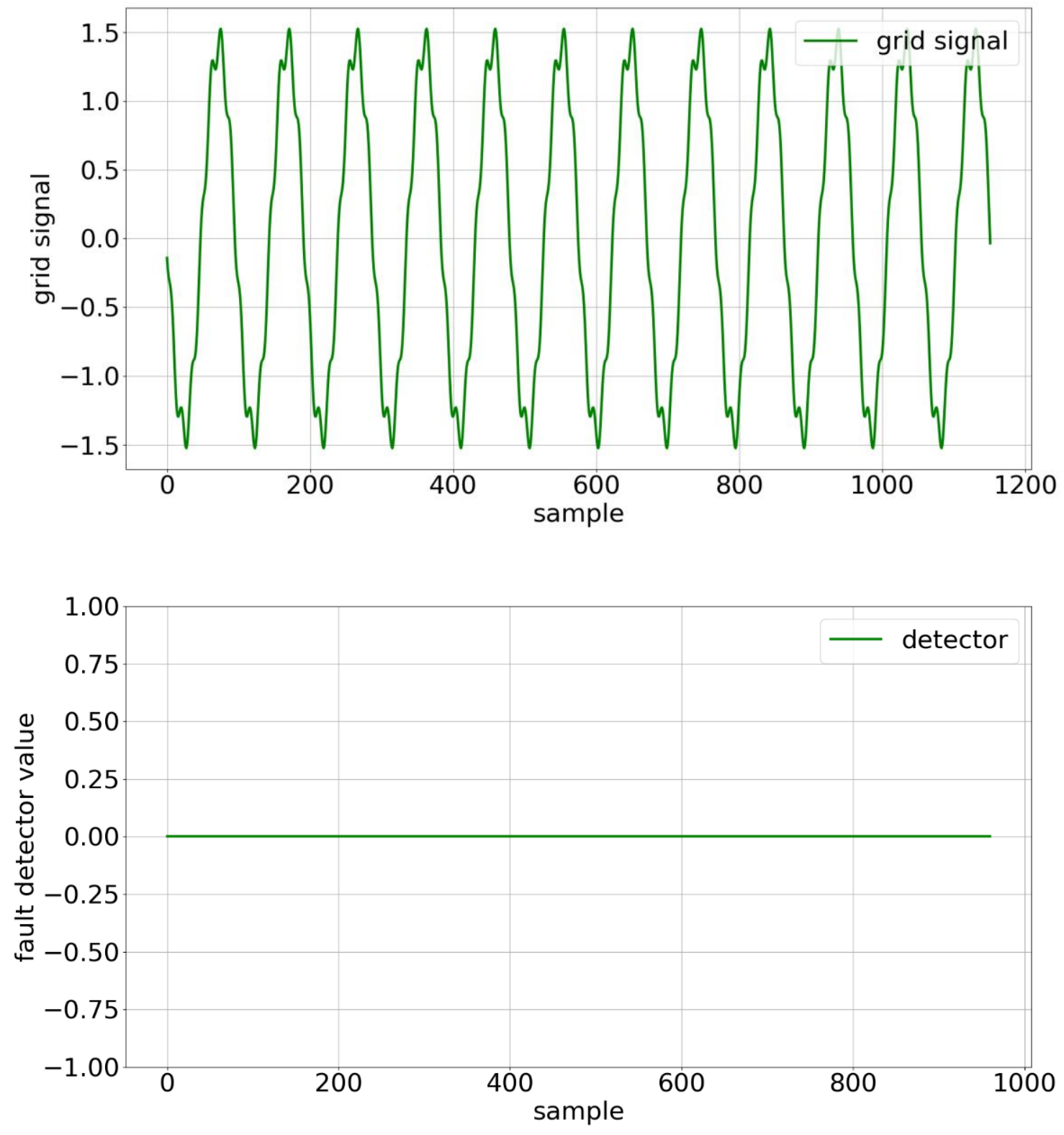

Figure 10 detector output - grid signal with $10 \%$ 7th harmonic 

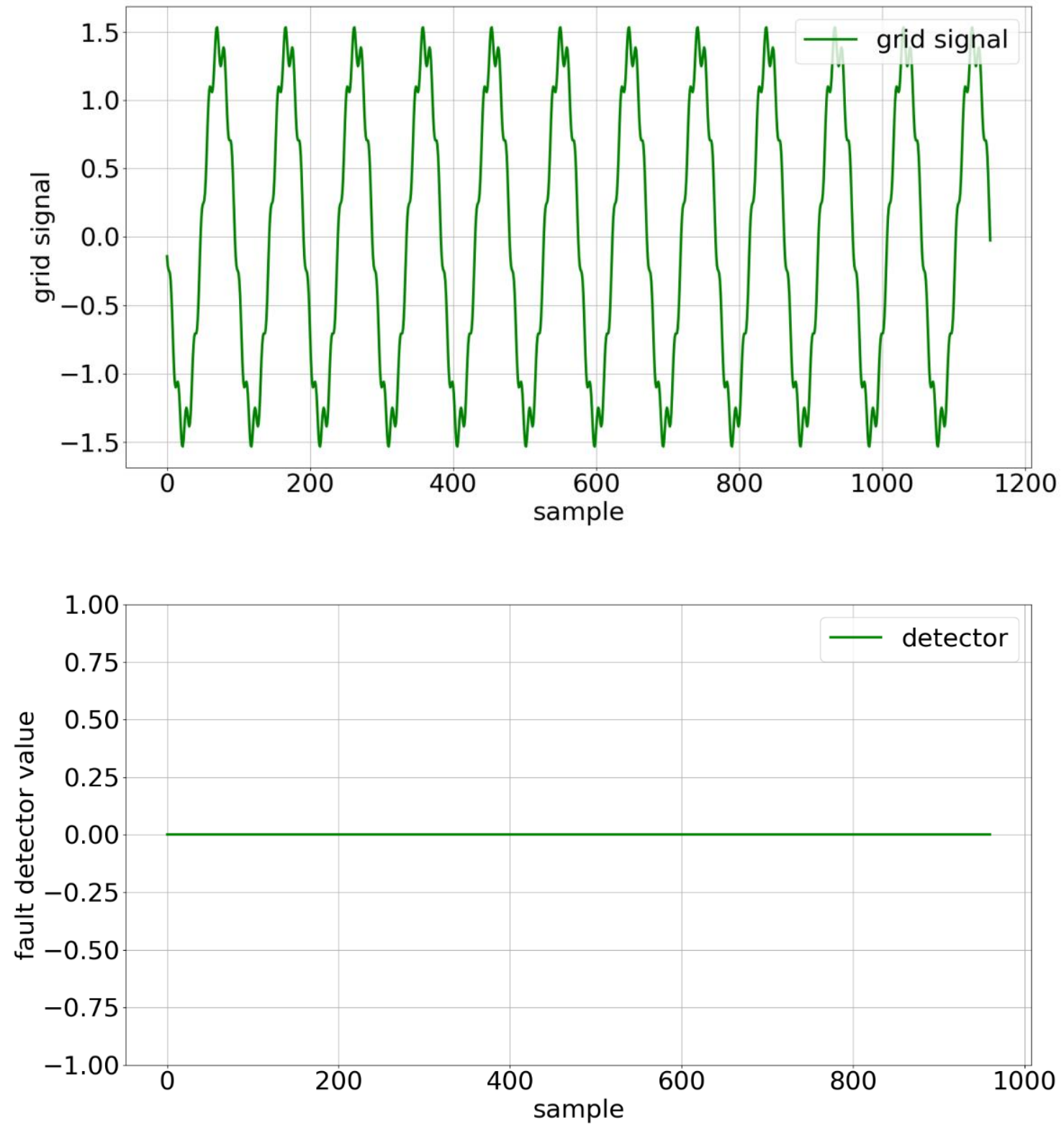

Figure 11 detector output - grid signal with $10 \% 9$ th harmonic 


\subsubsection{Phase angle step change test}

In the Aug. 2016 large scale solar tripping event, significant frequency measurements errors are caused by the phase angle step change during a system fault. As discussed earlier, the phase angle step change will introduce a break point to the original continuous and smooth waveform, and this break point will introduce significant measurement errors. Therefore, successful detection of the occurrence of the break point is critical to minimize the measurement errors, ultimately contributing to fault-tolerant measurements.

To perform the testing, a total number of 143 tests have been done. The phase angle step change in each test is as follows:

$$
\theta=-360+5 * i, \quad i=1,2, \ldots, 143(6)
$$

Figure 12 shows the value of detector with respect to the phase angle step change from -360 degree to 360 degree at a step change of 5 degrees, as given in (6).

Figure 13 shows the frequency measurement errors with respect to the phase angle step change. From the two figures, we can find a proportional relationship between fault detector output value and frequency measurement errors. This will provide us the foundation to limit the frequency measurement errors by tuning the threshold of the fault detector.

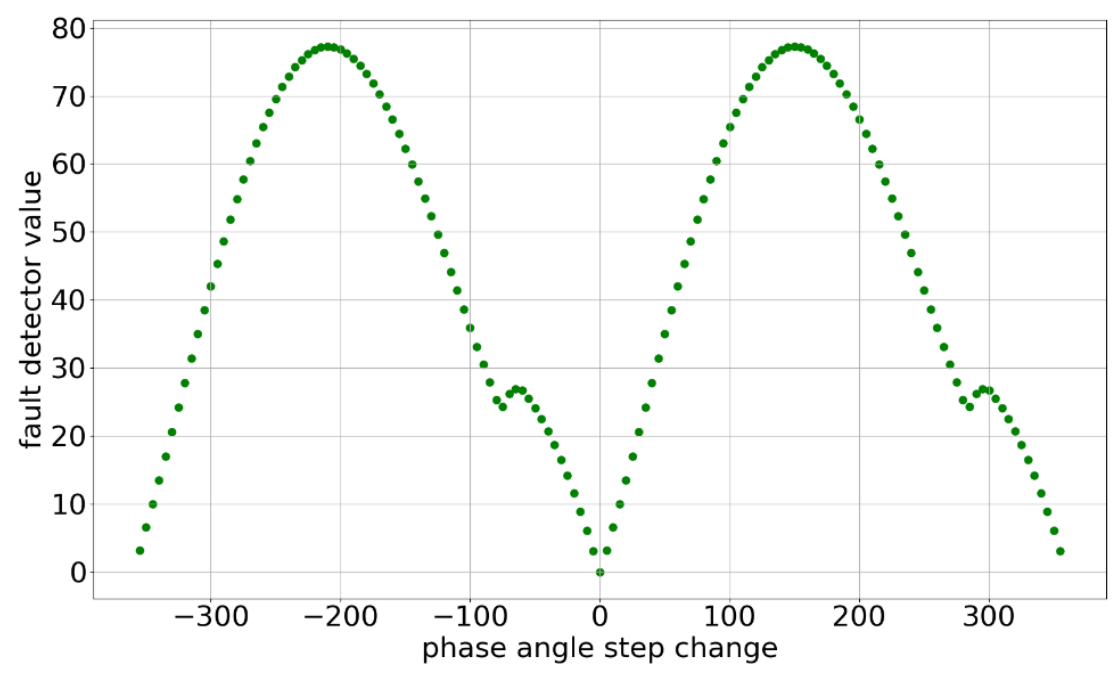

Figure 12 detector output - grid signal with different phase angle step change 


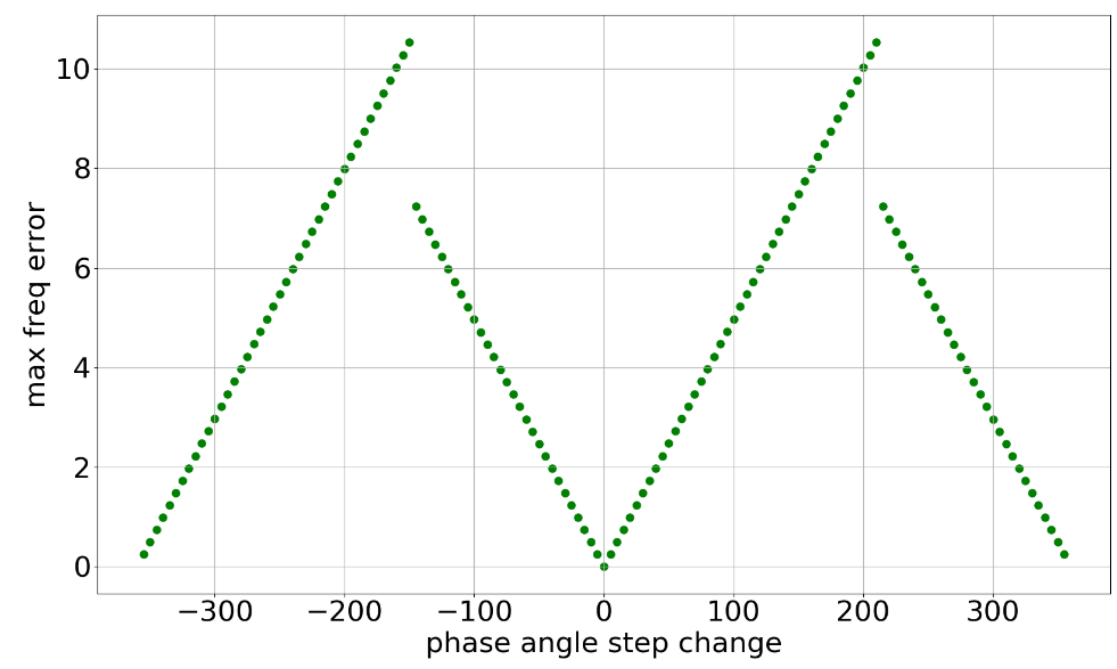

Figure 13 Frequency - grid signal with phase angle step change

\subsubsection{Development of an adaptive frequency estimation algorithm}

In last section, we develop a grid transient detector. It has been successfully tested, and it can detect the transients of grid signals, particularly the step change of the grid signals. The second step is the frequency estimator, and it will be able to adapt to the grid transient events based on the detection results in the first step. A high-level flowchart of the second step is shown in Figure 14. The detection results from grid transient detector will be compared to a transient threshold. If the result exceeds the threshold, which means a system transient is detected, the output of adaptive frequency estimator will be locked to the latest frequency measurements. When the outputs of the grid transient detector are lower than the threshold for one fundamental frequency cycle (1/60 second for $60 \mathrm{~Hz}$ system), the extra fast frequency estimator, using a short estimation window of 2 cycles, will start to estimate the grid frequency. If the outputs of the transient detector fall below the threshold for 2.25 cycles, the fast frequency estimator, using an estimation window of 3 cycles will start to estimate the grid frequency. When the output of the transient detector is below the threshold equal or greater than 4.75 cycles, the classical frequency estimation algorithm with an estimation window size of 5.75 cycles will start to provide frequency measurements.

First, the threshold used for determining the occurrence of system transients needs to be determined. As discussed in last section, we did phase angle step change test, and the phase angle step change in each test is as follows:

$$
\theta=-360+5 * i, \quad i=1,2, \ldots, 143(7)
$$

Additionally, as the step change can occur at any time in one cycle, so the phase angle step change has been tested at different phase angles from 0 degree to 360 degree. 


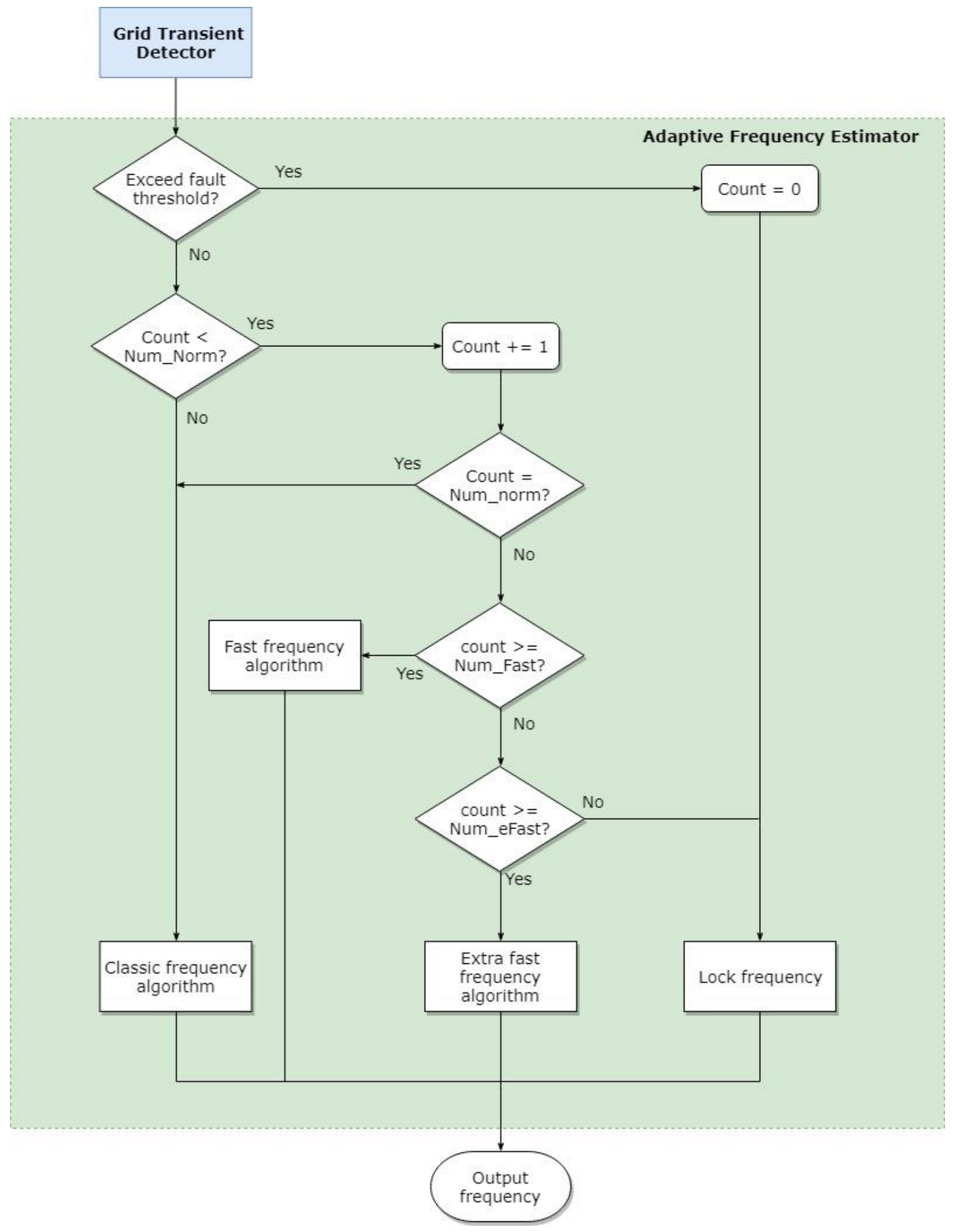

Figure 14 Architecture of the proposed Adaptive Frequency Estimator 


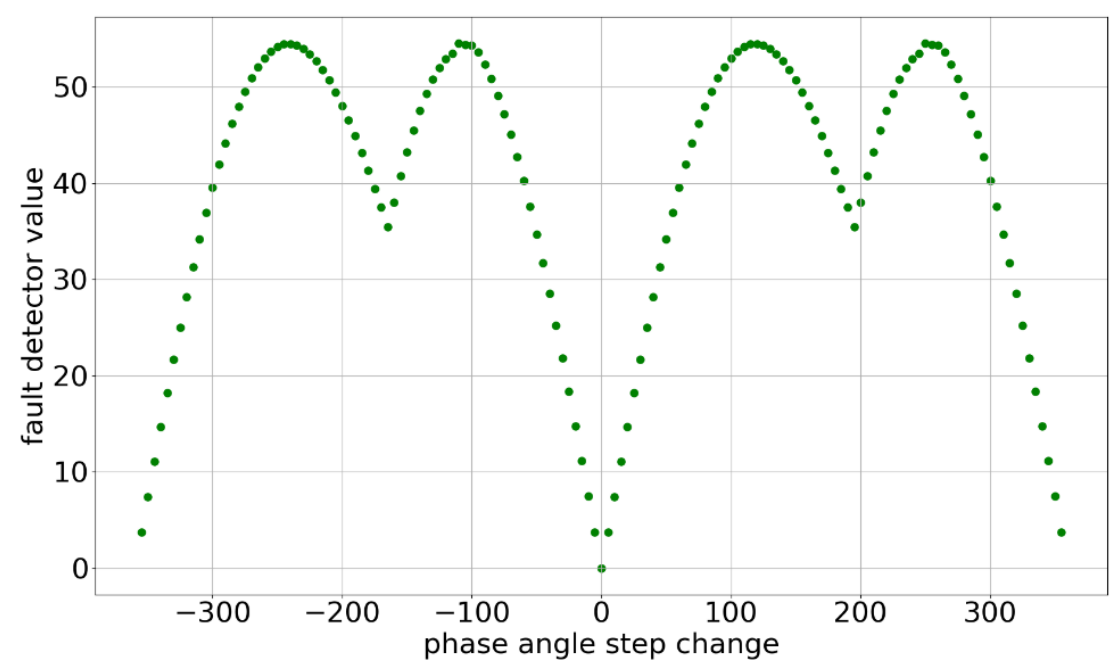

(a) Phase angle 0 degree at the time of event

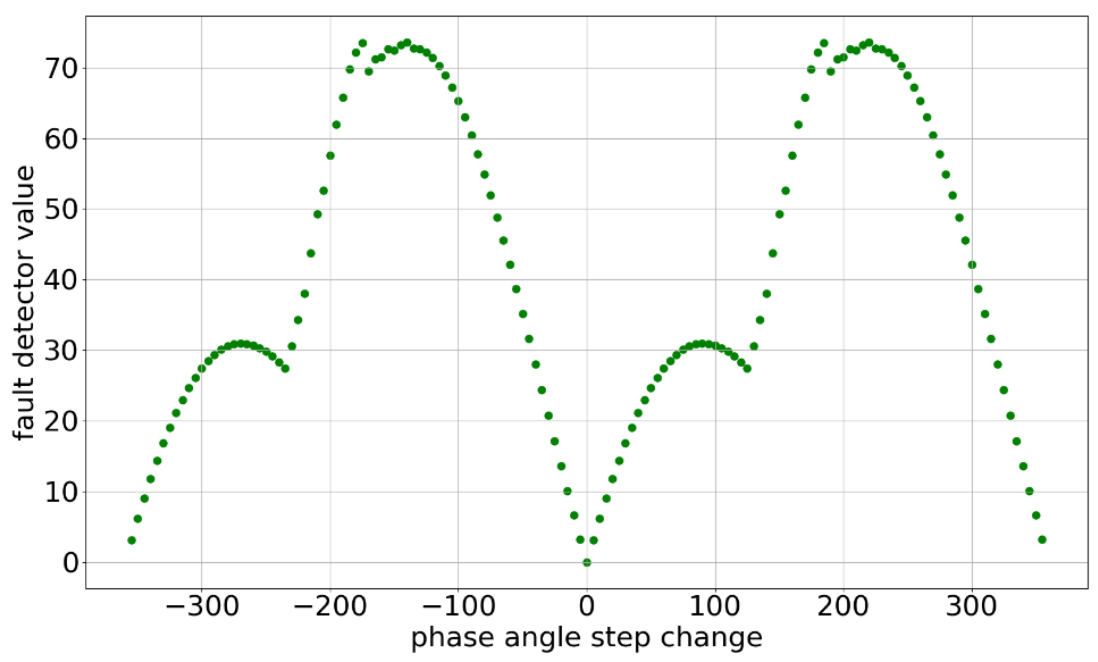

(b) Phase angle 45 degree at the time of event 


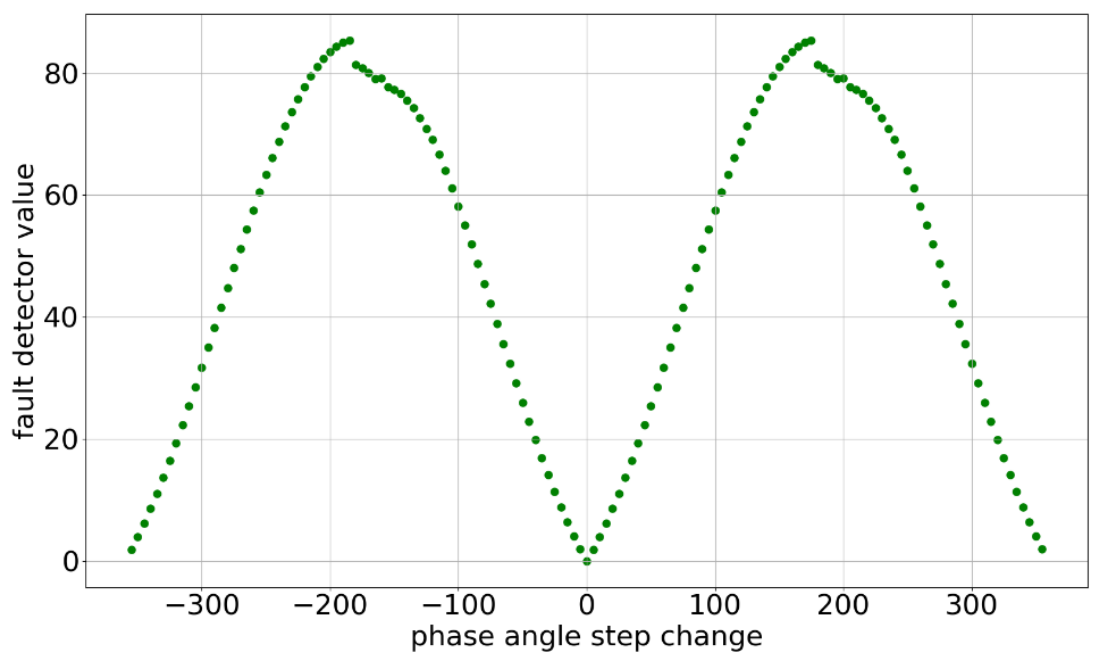

(c) Phase angle 90 degree at the time of event

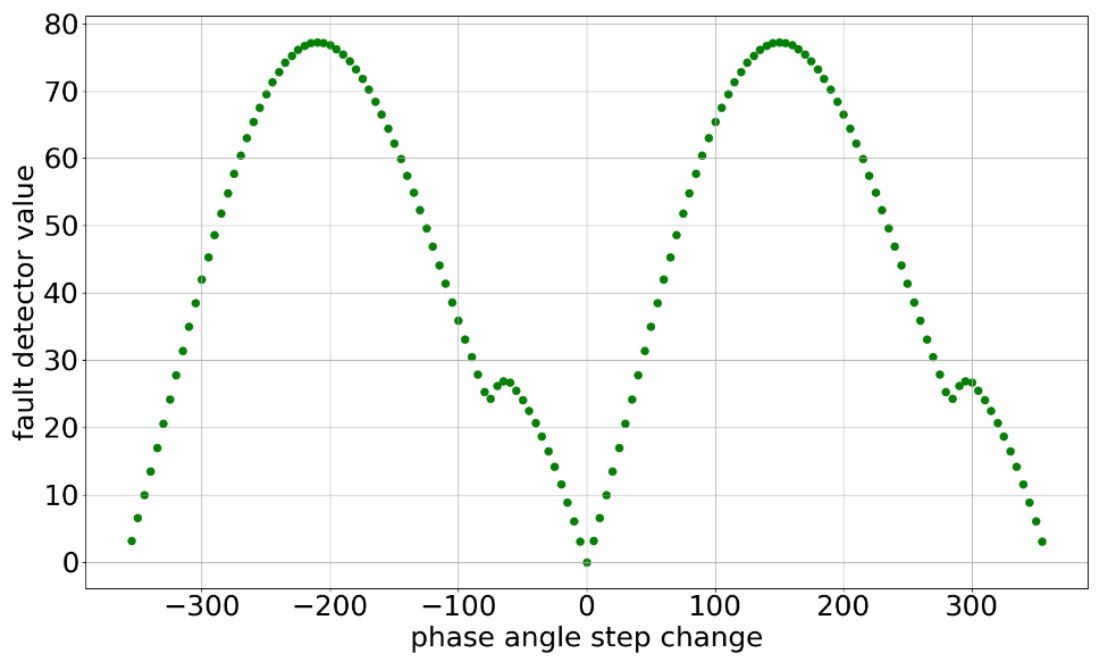

(d) Phase angle 135 degree at the time of event 


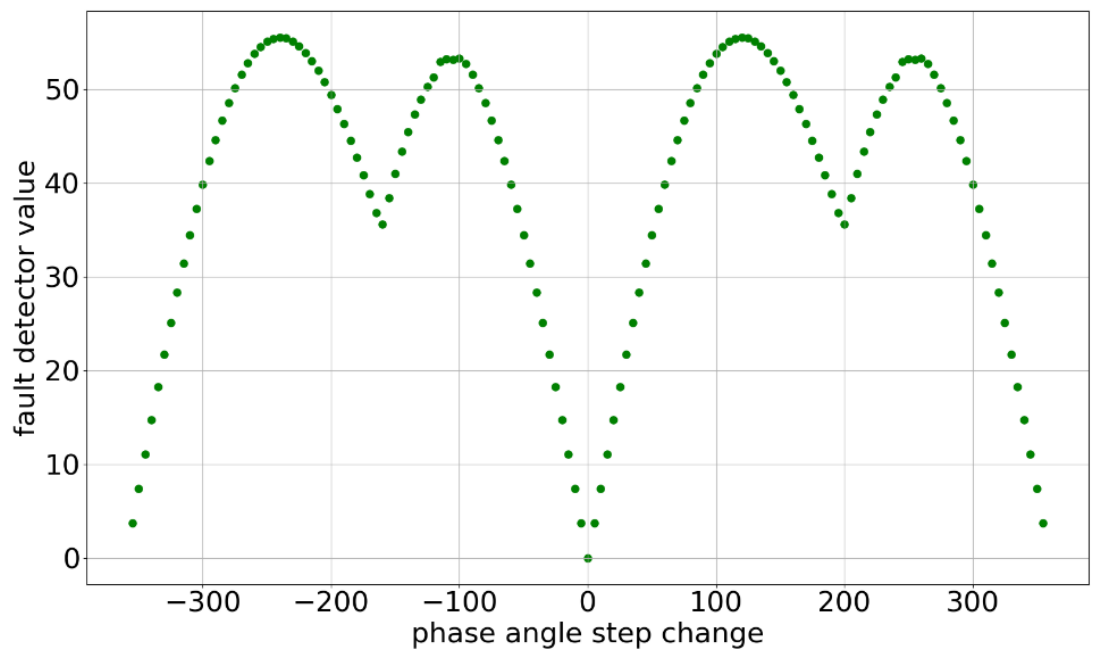

(e) Phase angle 180 degree at the time of event

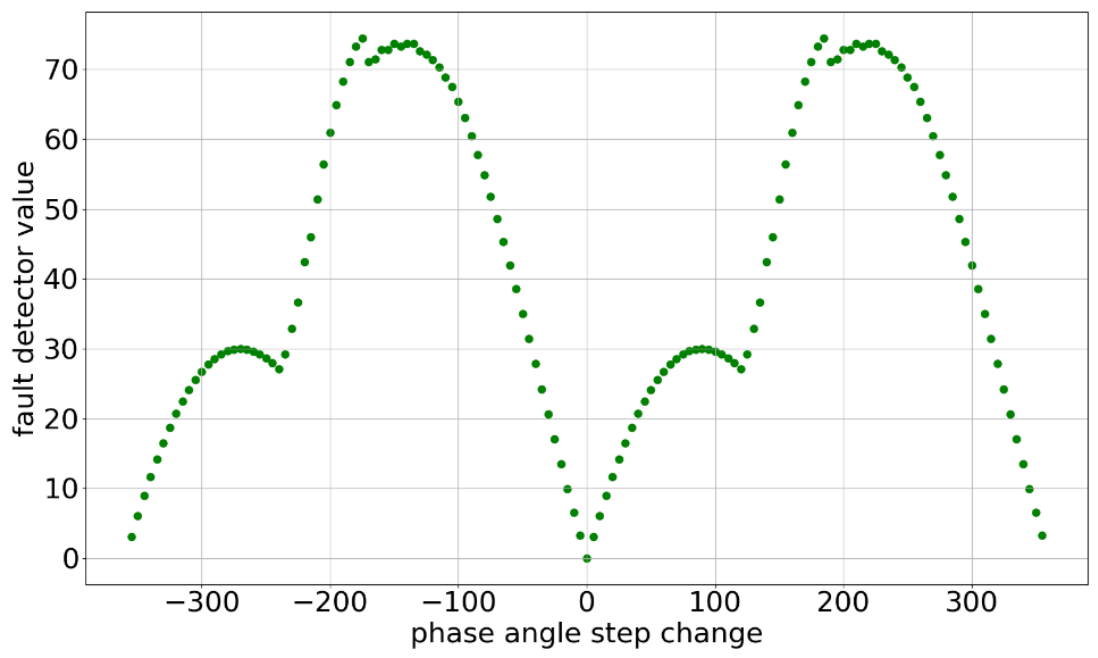

(f) Phase angle 225 degree at the time of event 


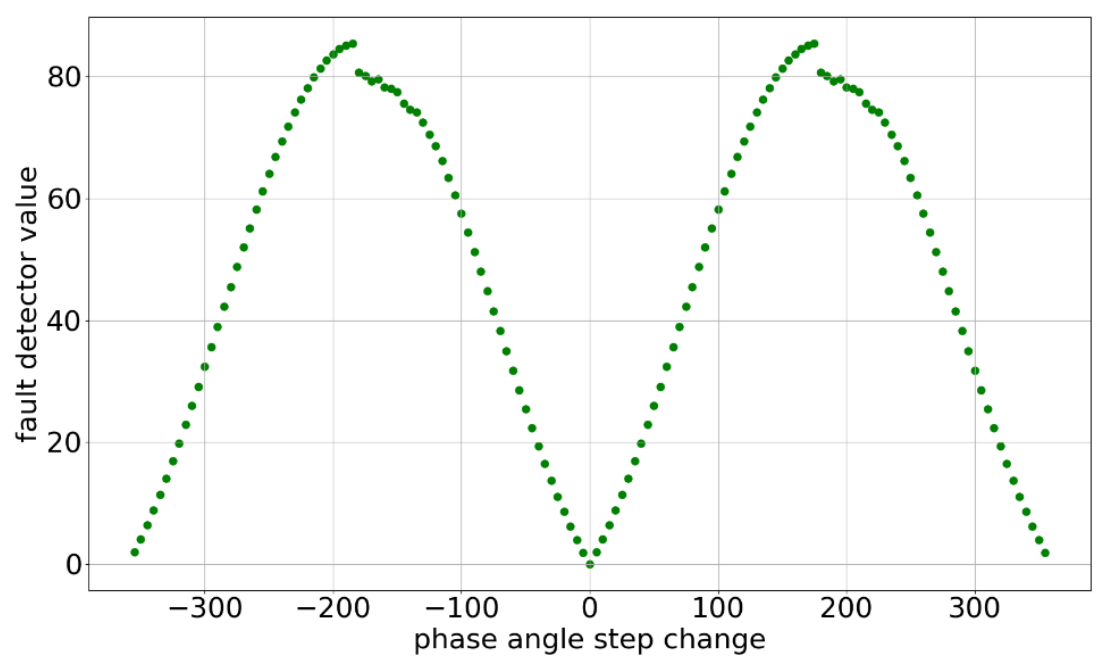

(g) Phase angle 270 degree at the time of event

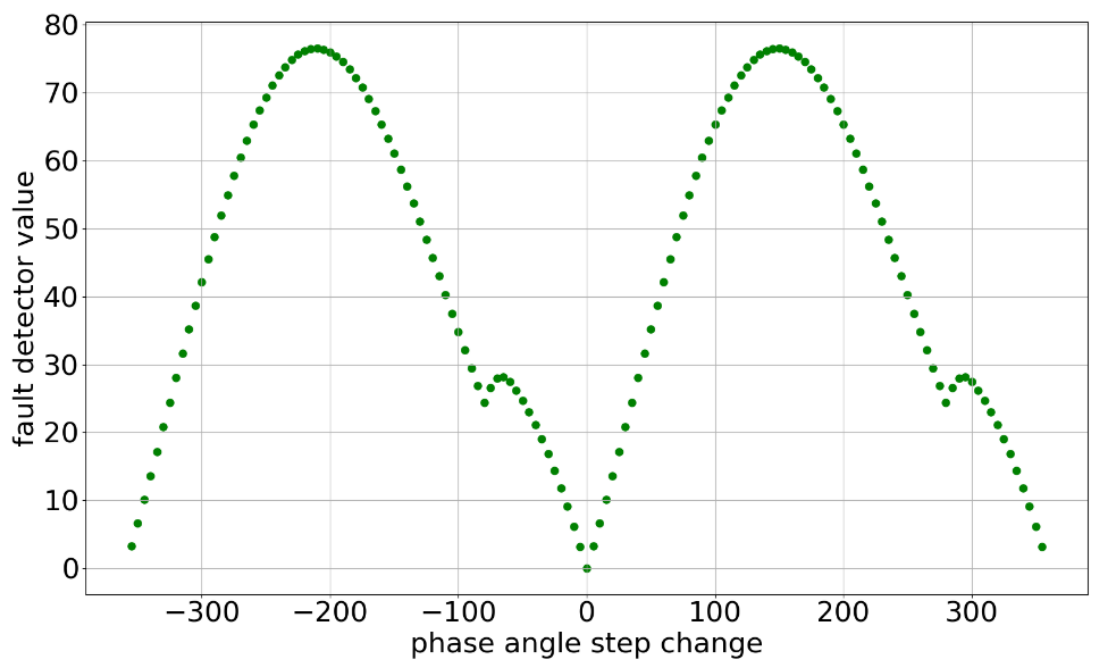

(h) Phase angle 315 degree at the time of event

Figure 15 detector output - grid signal with different phase angle step change

From those figures, it can be seen there is a proportional relationship between fault detector output value and the absolute value of the phase angle step change when the change within a certain range. On the other hand, there is also a proportional relationship between the absolute value of the phase angle step change and frequency measurement errors. Therefore, the threshold can be determined by checking the detector values of different phase angles at the time of event with the phase angle step change in a small range. Here phase angle step change from -20 degree to 20 degree is chosen. Figure 16 shows the fault detector value with respect to the different phase angles at the time of event. The fault threshold should be determined by the lowest value. In each test, we monitor whether the frequency deviation is over $0.5 \mathrm{~Hz}$. If that happens, we will find the maximum value of detector's output until when the frequency deviates to $0.5 \mathrm{~Hz}$ and save this value for next step. Next, as we run from -20 degree to 20 degree, we will find the minimum value from a set of the maximum values, and we plot this minimum value as a "dot" in Figure 
16. At last, we choose the minimum value from all the "dots" and make it as our threshold value. By doing this, the transient detector will make sure can detect all the phase angle step change event that cause over $0.5 \mathrm{~Hz}$ frequency errors.

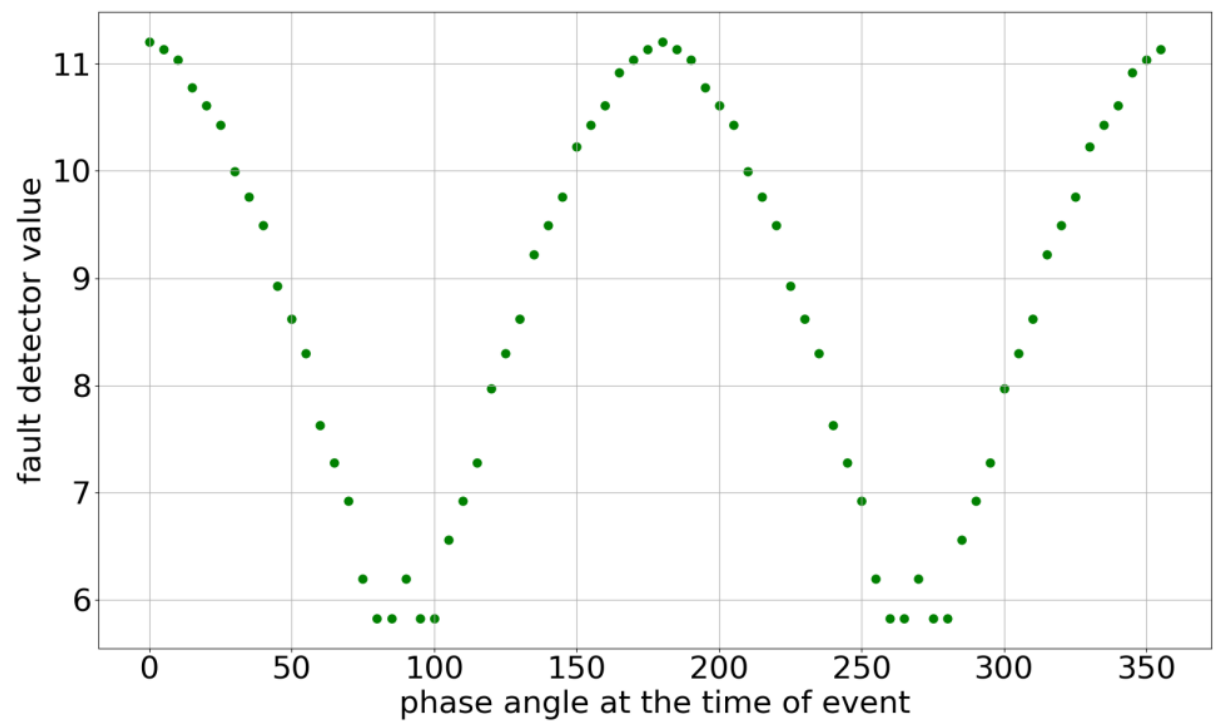

Figure 16 detector output -grid signal with different phase angle at the time of event (sampling frequency: $5760 \mathrm{~Hz}$, signal frequency: $60 \mathrm{~Hz}$ )

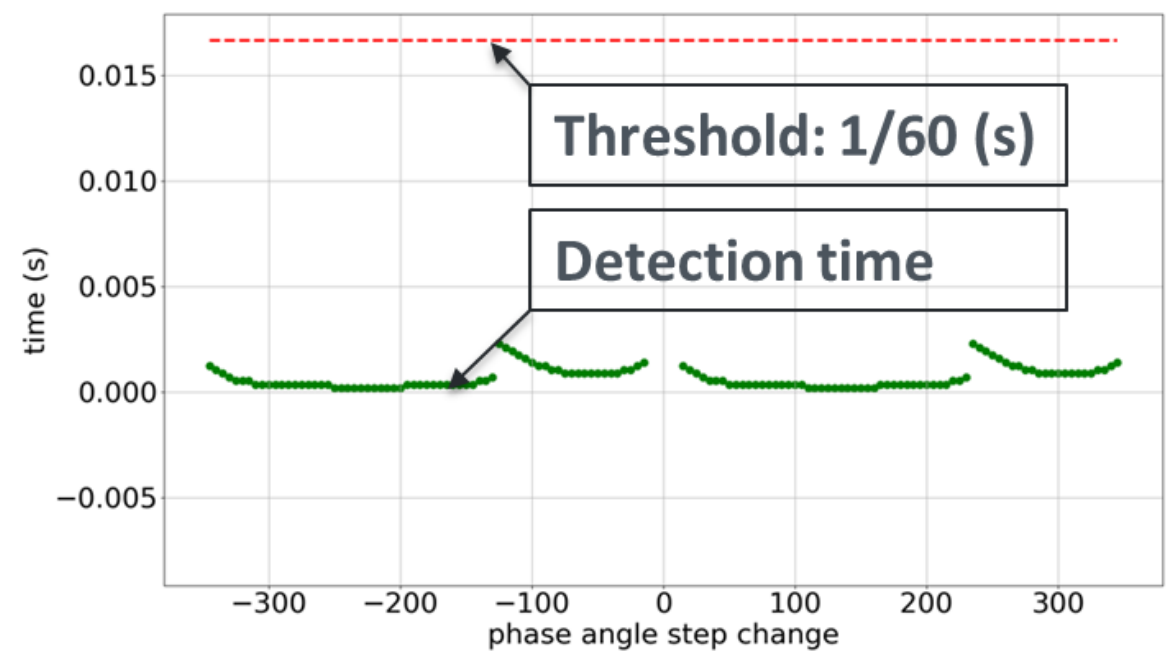

Figure 17 Detection time at different phase angle step change

\section{Milestone \#1 tests}

Figure 17 shows the test results for Milestone \#1. The total number of tests is 143 . Out of the total 143 tests, there are 134 tests that cause over $0.5 \mathrm{~Hz}$ frequency errors. The transient detector successfully detected all the tests, and Figure 17 shows the detection time for each test. We can see the detection time of all tests are below 1/60 second. 


\begin{tabular}{|l|l|l|l|}
\hline Milestone & Metric Definition & $\begin{array}{l}\text { Finished } \\
\text { Date }\end{array}$ & $\begin{array}{l}\text { Success } \\
\text { Value }\end{array}$ \\
\hline 1 & $\begin{array}{l}\text { Occurrence of system transient events that could cause over } 0.5 \mathrm{~Hz} \\
\text { frequency measurement error is detected within one fundamental } \\
\text { frequency cycle }(1 / 60 \text { second) at a success rate of above } 95 \% \text { by } \\
\text { measuring the time from transient occurrence to successful } \\
\text { detection. }\end{array}$ & $1 / 31 / 2019$ & $\mathbf{1 0 0 \%}$ \\
\hline
\end{tabular}

As we discussed in last section, the detector equation could be represented as follows,

$$
\gamma(k)=\left|\sum_{k=0}^{N} x(k)-x(k-N)\right|(8)
$$

where $\mathrm{N}$ is the number of samples in one fundamental frequency cycle. Thus, the sampling frequency will be a dominant factor for the fault threshold value. And for a fixed sampling rate, the signal frequency and the signal magnitude will impact the fault threshold.

First, the relationship between the signal frequency and the fault threshold is studied. Figure 18 shows the fault threshold with respect to different signal frequency at a sampling rate of $5760 \mathrm{~Hz}$. The magnitude of signals tested here is 1 per unit. As shown in the figure, the fault threshold value decreases as the signal frequency increases and vice versa. As a result, for a fixed sampling rate, signal with higher frequency should be used to determine the threshold. Here signal of frequency $63 \mathrm{~Hz}$ is applied to determine the fault threshold.

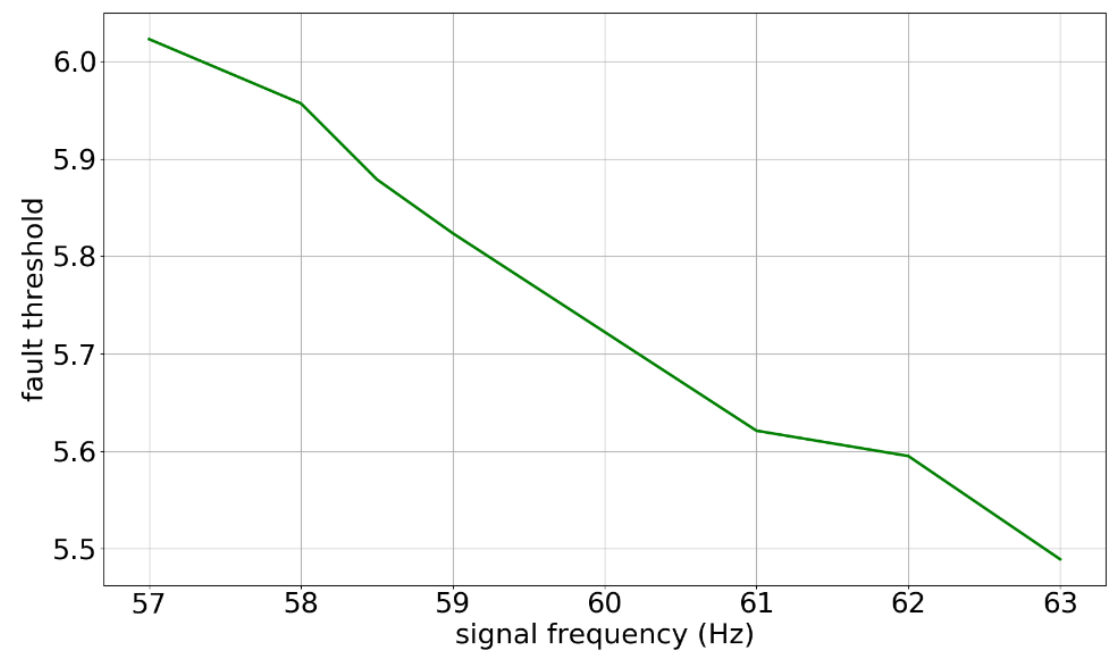

Figure 18 fault threshold value with different signal frequency (sampling rate: $5760 \mathrm{~Hz}$ )

Next, the relationship between the signal magnitude and the fault threshold is studied. The signals with different magnitude are tested. Figure 19 presents the fault threshold with respect to different signal magnitude at the same sampling frequency and signal frequency. 


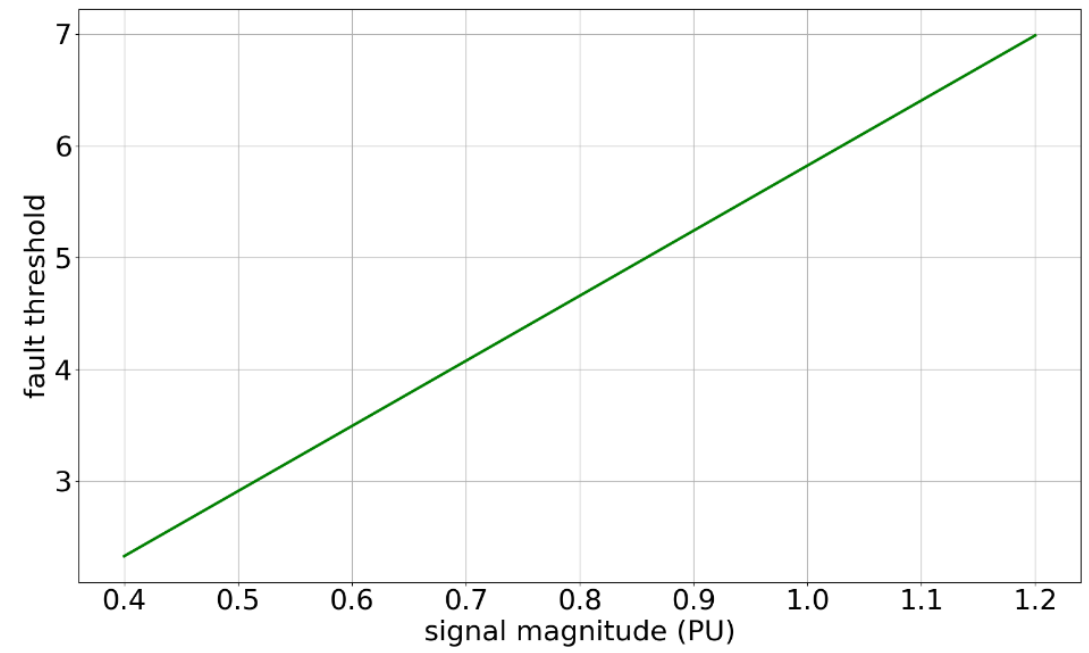

Figure 19 fault threshold value with different signal magnitude (sampling frequency: $5760 \mathrm{~Hz}$, signal frequency: $60 \mathrm{~Hz}$ )

The fault threshold is proportional to the per unit value of signal magnitude. Thus, for a specific sampling frequency, a base threshold $T H R_{B}$ is first determined by using signal of frequency $63 \mathrm{~Hz}$ with 1 per unit magnitude. Then, the threshold $T H R$ is updated by the per unit value of the tested signal magnitude

$$
T H R=T H R_{B} \times M_{P U}(9)
$$

As mentioned above, the sampling frequency will be an important factor to the fault threshold value. The base threshold value with respect to the different sampling frequency is shown in Figure 20. The threshold value is nearly proportional to the sampling frequency.

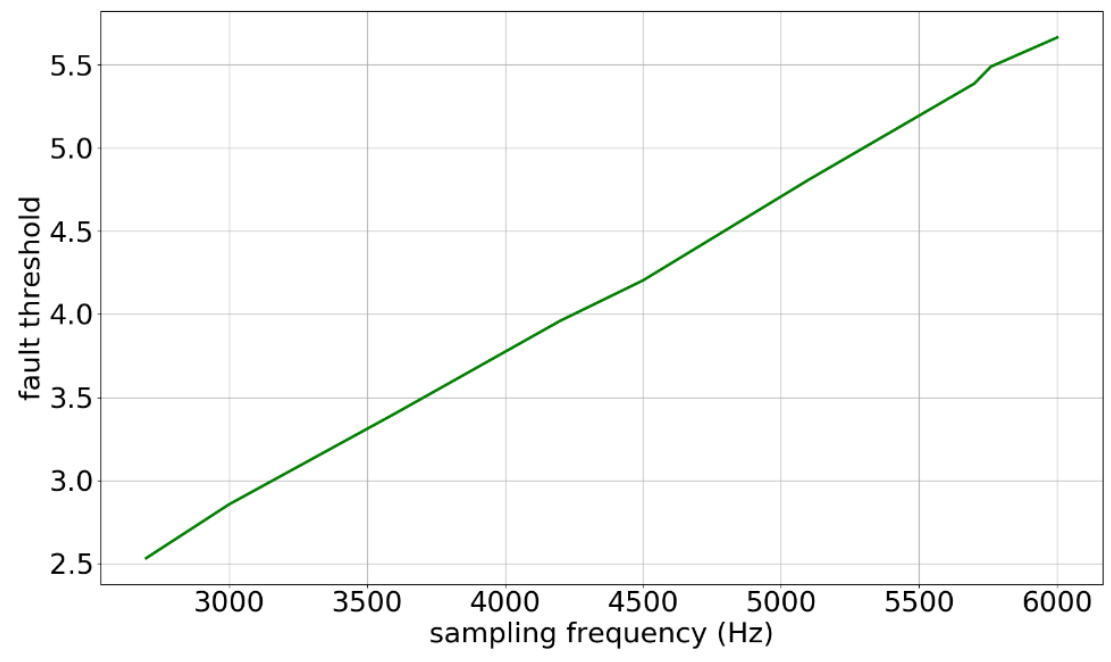

Figure 20 fault threshold value with different sampling frequency

\section{Milestone 2}

The fault-tolerant frequency estimator has been implemented using Python/C++ in Visual Studio 2017 for performance testing. Besides phase jumps, the test signals will be also distorted by noise, harmonics and voltage sag for a more practical evaluation. Selected test cases are listed in Table II, and results are presented below. The sampling frequency of all the test signals is $5760 \mathrm{~Hz}$. According to Figure 18 and considering some margin, 5 is set as the base fault threshold. 
Table II Performance Test Cases for Fault-tolerant Frequency Estimator

\begin{tabular}{|c|c|c|c|c|c|c|}
\hline Case & $\begin{array}{c}\text { Signal } \\
\text { frequency (Hz) }\end{array}$ & $\begin{array}{c}\text { Noise } \\
(\mathrm{dB})\end{array}$ & THD & $\begin{array}{c}\text { Voltage } \\
\text { drop (\%) }\end{array}$ & $\begin{array}{c}\text { Phase angle } \\
\text { step change } \\
\text { (degree) }\end{array}$ & Max $\Delta f$ in 2 cycles (Hz) \\
\hline 1 & 60 & 70 & $10 \%$ & $50 \%$ & 180 & 0.0005 \\
\hline 2 & 60 & 70 & $10 \%$ & $50 \%$ & 45 & 0.0005 \\
\hline 3 & 60 & 70 & $10 \%$ & $50 \%$ & 90 & 0.001 \\
\hline 4 & 60 & 70 & $10 \%$ & $50 \%$ & 225 & 0.0005 \\
\hline 5 & 60 & 70 & $10 \%$ & $50 \%$ & 270 & 0.0011 \\
\hline 6 & 60 & 50 & $10 \%$ & $50 \%$ & 180 & 0.0025 \\
\hline 7 & 60 & 70 & $20 \%$ & $50 \%$ & 180 & 0.0007 \\
\hline 8 & 60 & 70 & $10 \%$ & $90 \%$ & 180 & 0.005 \\
\hline 9 & 60 & 50 & $20 \%$ & $90 \%$ & 180 & 0.013 \\
\hline 10 & 59 & 70 & $10 \%$ & $50 \%$ & 180 & 0.037 \\
\hline 11 & 59 & 50 & $20 \%$ & $90 \%$ & 180 & 0.008 \\
\hline 12 & 59.5 & 70 & $10 \%$ & $50 \%$ & 180 & 0.014 \\
\hline 13 & 59.5 & 50 & $20 \%$ & $90 \%$ & 180 & 0.008 \\
\hline 14 & 60.5 & 70 & $10 \%$ & $50 \%$ & 180 & 0.013 \\
\hline 15 & 60.5 & 50 & $20 \%$ & $90 \%$ & 180 & 0.018 \\
\hline 16 & 61 & 70 & $10 \%$ & $50 \%$ & 180 & 0.032 \\
\hline 17 & 61 & 50 & $20 \%$ & $90 \%$ & 180 & \\
\hline
\end{tabular}

First, signals of frequency $60 \mathrm{~Hz}$ with $70 \mathrm{~dB}$ noise, $10 \%$ harmonics (including $3^{\text {rd }}, 5^{\text {th }}, 7^{\text {th }}, 11^{\text {th }}$, and $13^{\text {th }}$ order harmonics), and 50\% voltage magnitude step down with different phase jumps are considered for testing, the results are shown in Figure 21 to Figure 26. Take case 1 as an example, at the time of phase jump event, the phase angle step change is 180 degree, and voltage drops to $50 \% .10 \%$ harmonics is added and voltage magnitude is ramping up when the event starts. This case is also considered as a base case. Figure 21 presents the grid signal and frequency results by the classical frequency estimator and the proposed fault-tolerant frequency estimator. The zoomed-in estimated frequency by the fault-tolerant algorithm is shown in Figure 22, and the maximum frequency measurement errors is only $0.0005 \mathrm{~Hz}$ in 2 cycles following the phase-jump event, which is far below $0.05 \mathrm{~Hz}$ as we proposed in Milestone \#2. To make the test more complete, the signals with 45 degree, 90 degree, 225 degree and 270 degree phase jumps are also tested, and results are shown in Figure 23 - Figure 26. 

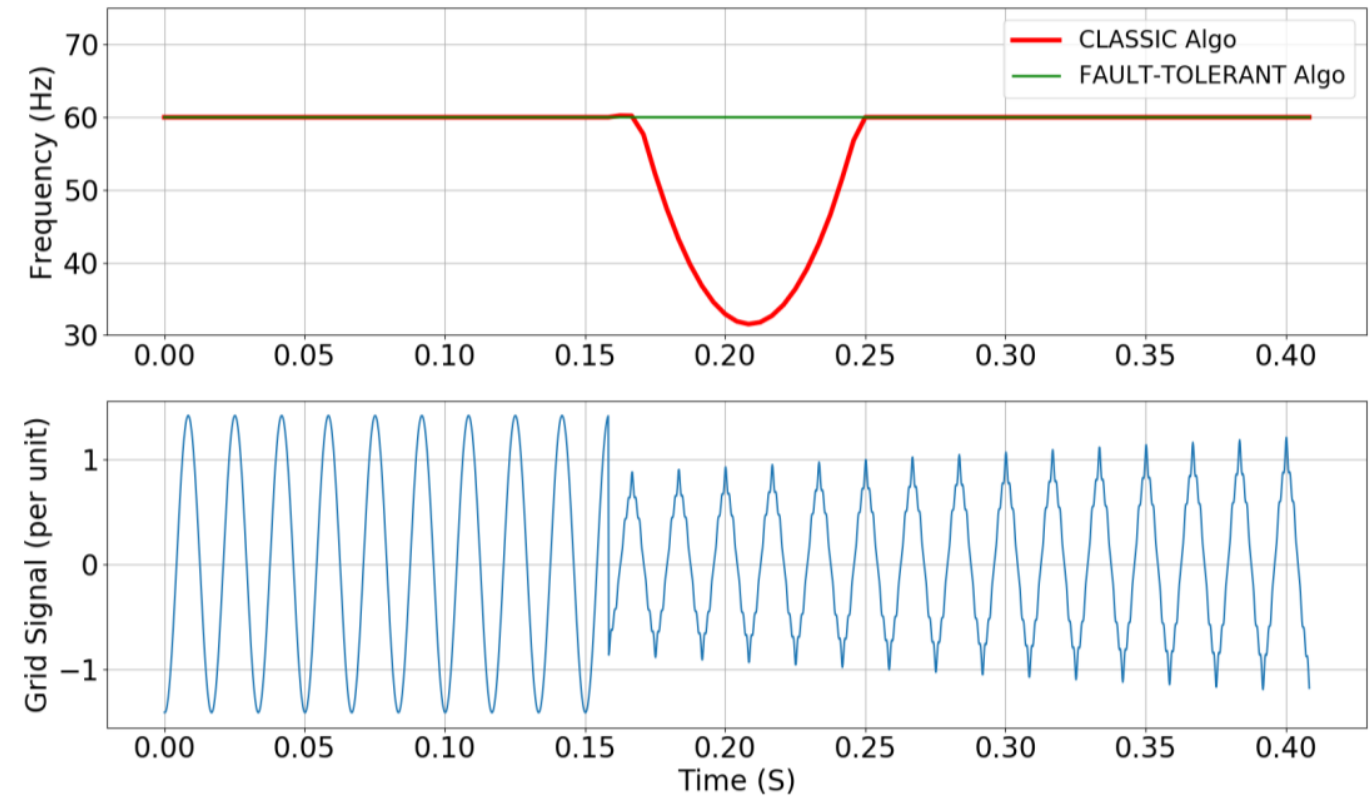

Figure 21 testing signal and results of case 1: 180-degree phase jump

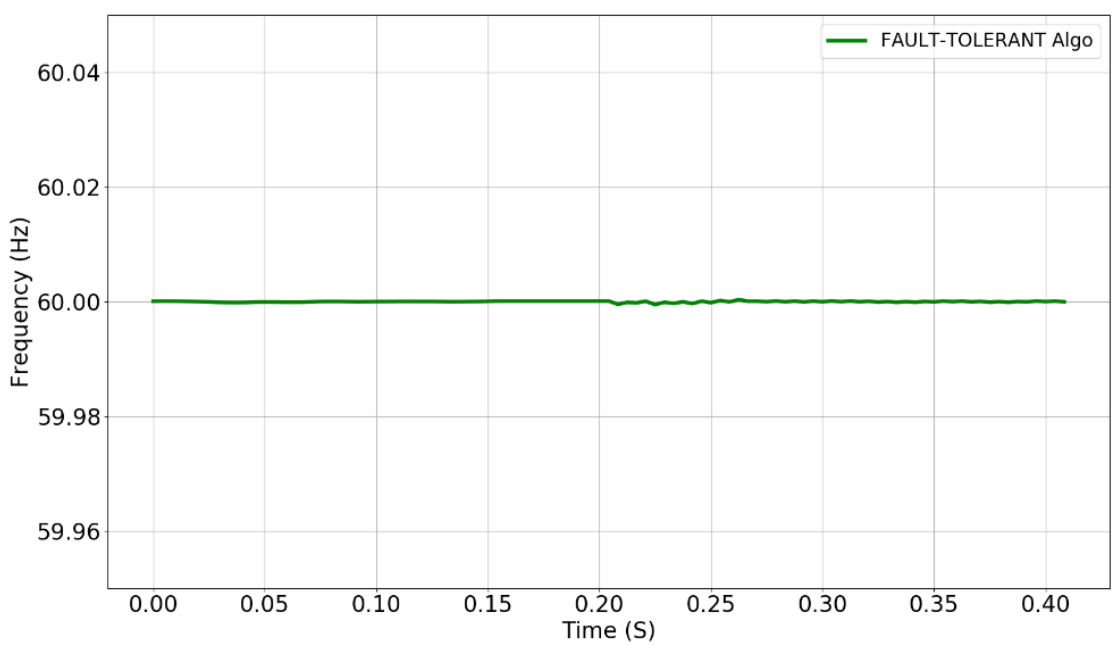

Figure 22 zoomed in estimated frequency by the fault-tolerant algorithm of case 1 

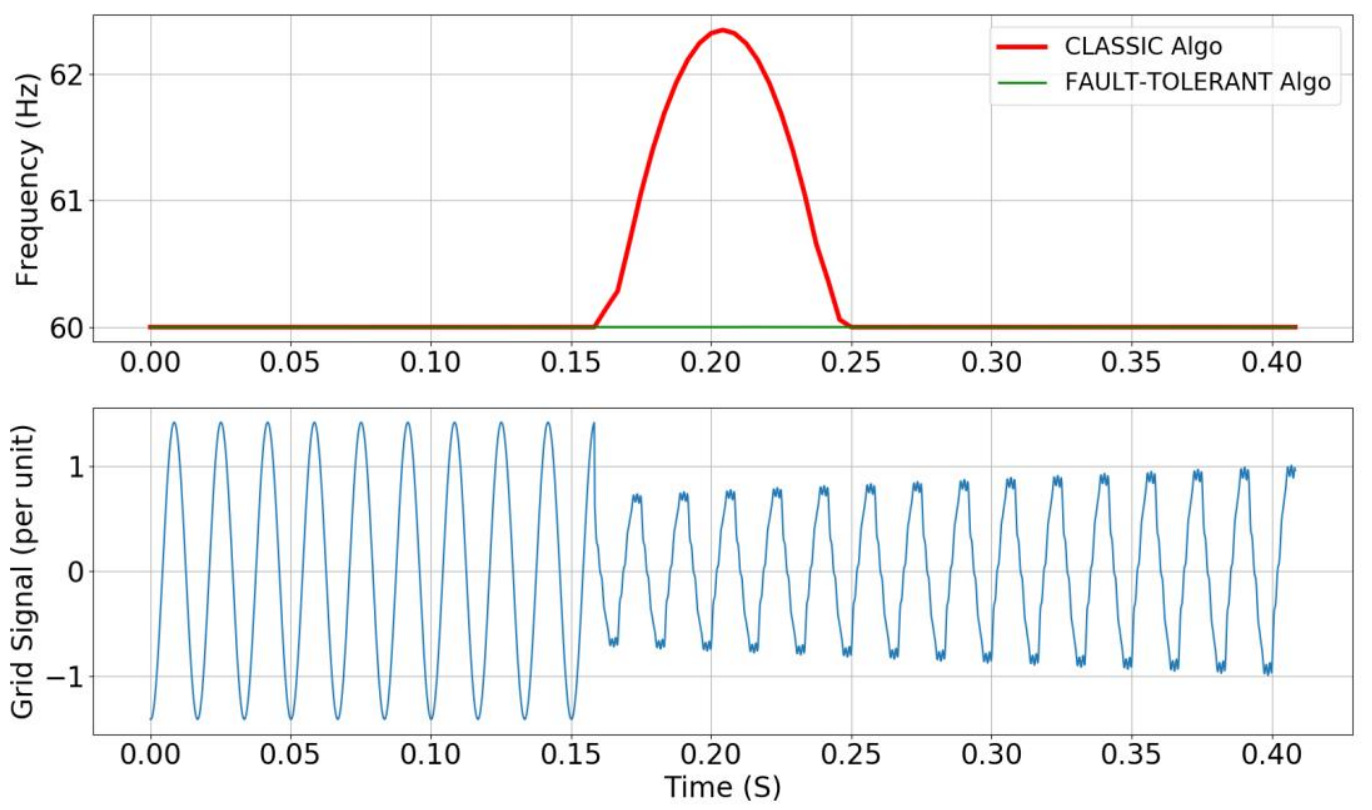

Figure 23 testing signal and results of case 2: 45-degree phase jump
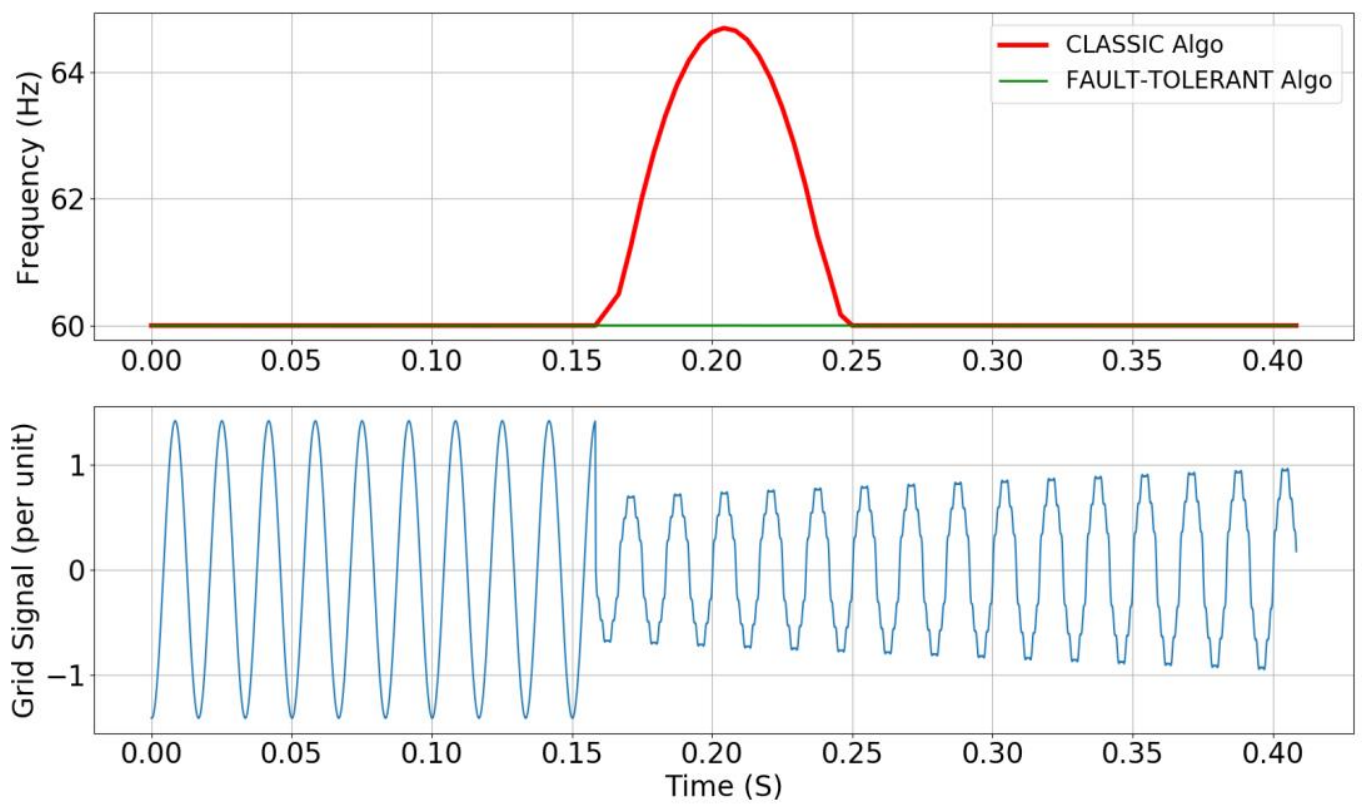

Figure 24 testing signal and results of case 3: 90-degree phase jump 

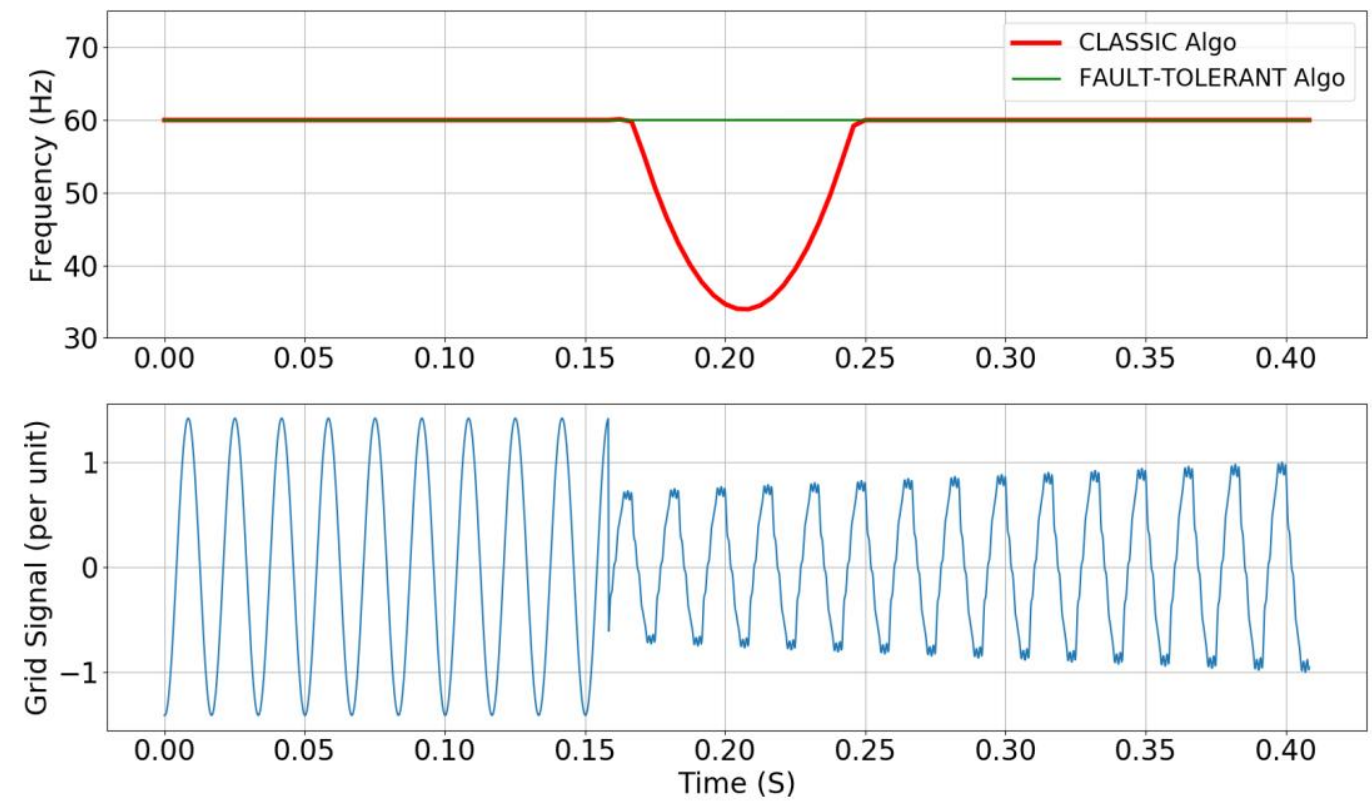

Figure 25 testing signal and results of case 4: 225-degree phase jump
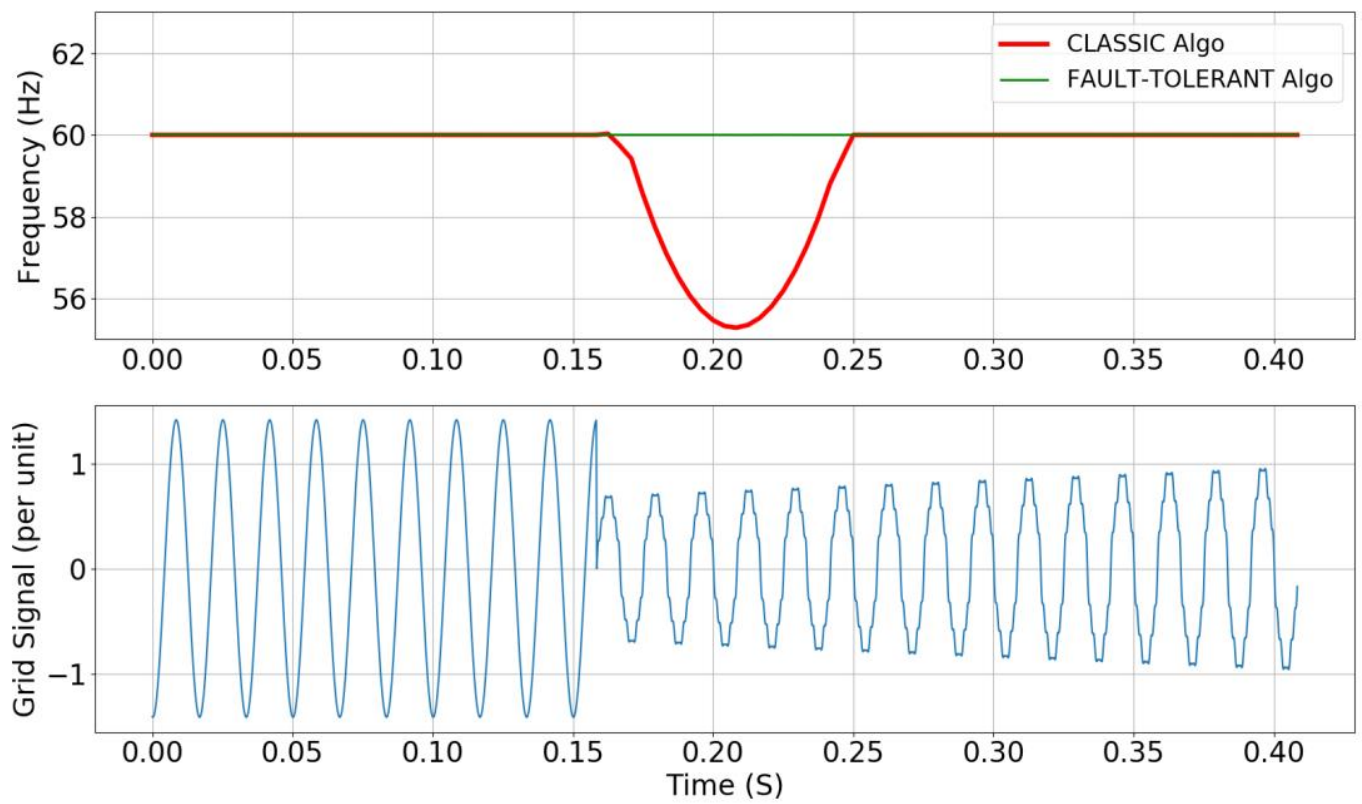

Figure 26 testing signal and results of case 5: 270-degree phase jump

Based on case 1, we have also changed the noise level, harmonics level and/or voltage drop level to make the test cases even worse and see to evaluate the performance of the fault-tolerant frequency estimator. The details of each test are listed in Table II, and results are shown below. Among those test cases, case 9 is the worst one, with $50 \mathrm{~dB}$ noise, $20 \%$ harmonics and $90 \%$ voltage drop at the time of phase jump, and 
it can be seen from Figure 30 that the fault-tolerant frequency estimator still works well, meeting our milestone 2 .
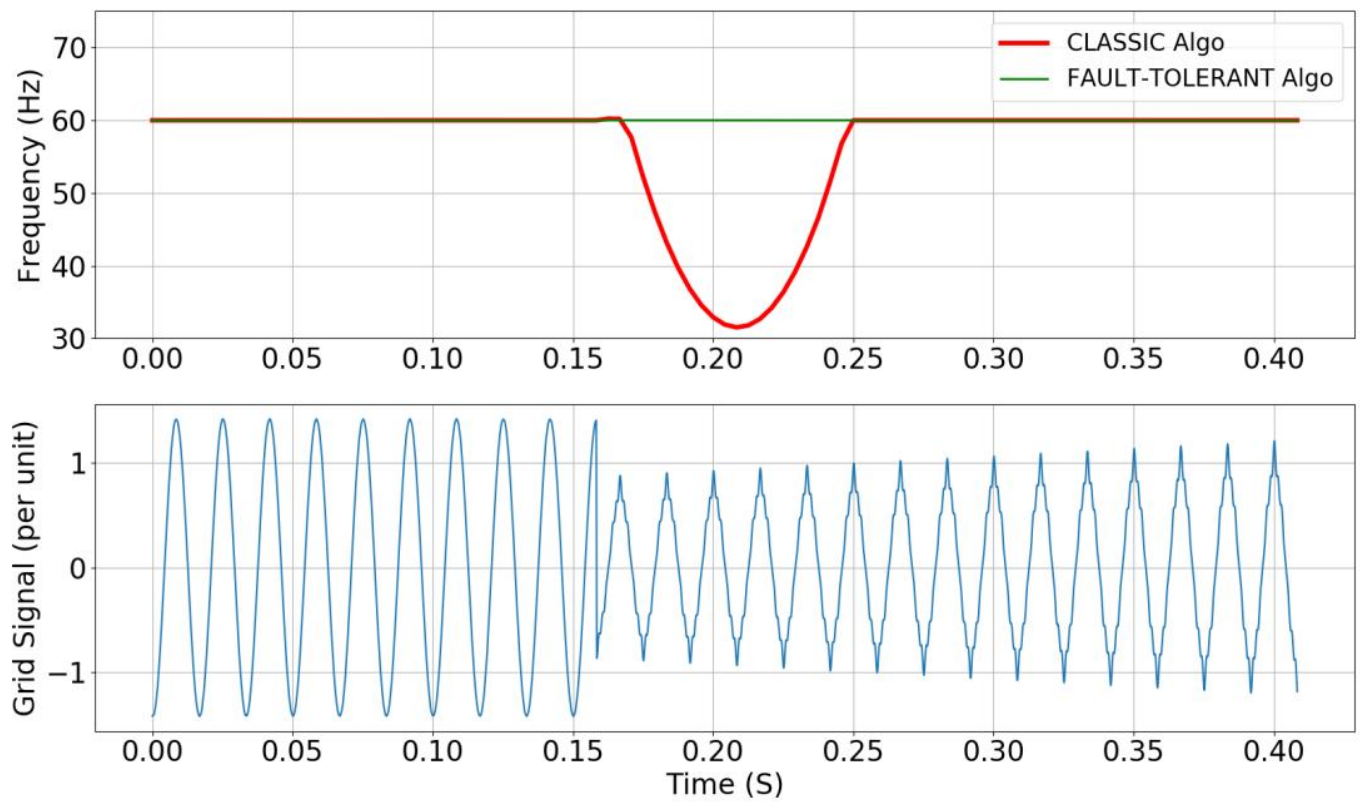

Figure 27 testing signal and results of case 6
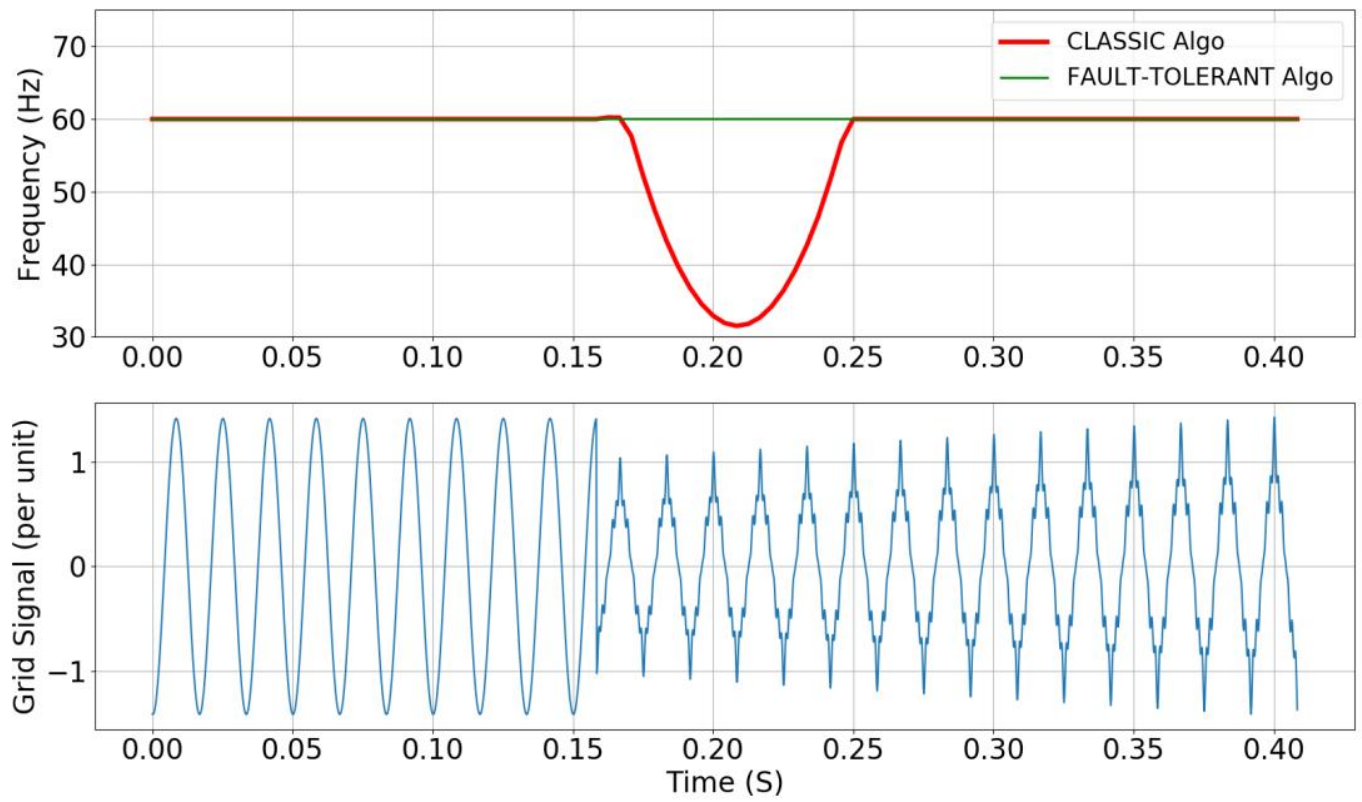

Figure 28 testing signal and results of case 7 

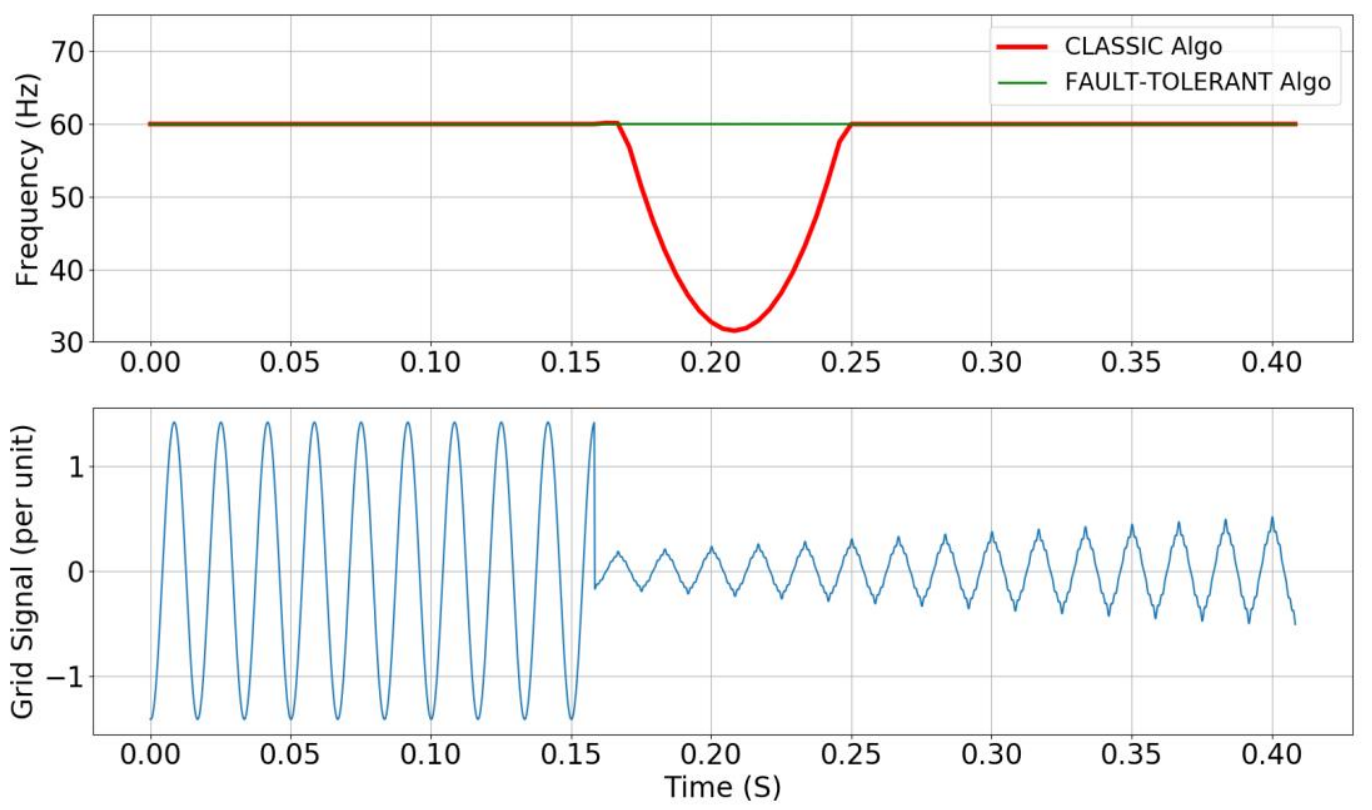

Figure 29 testing signal and results of case 8
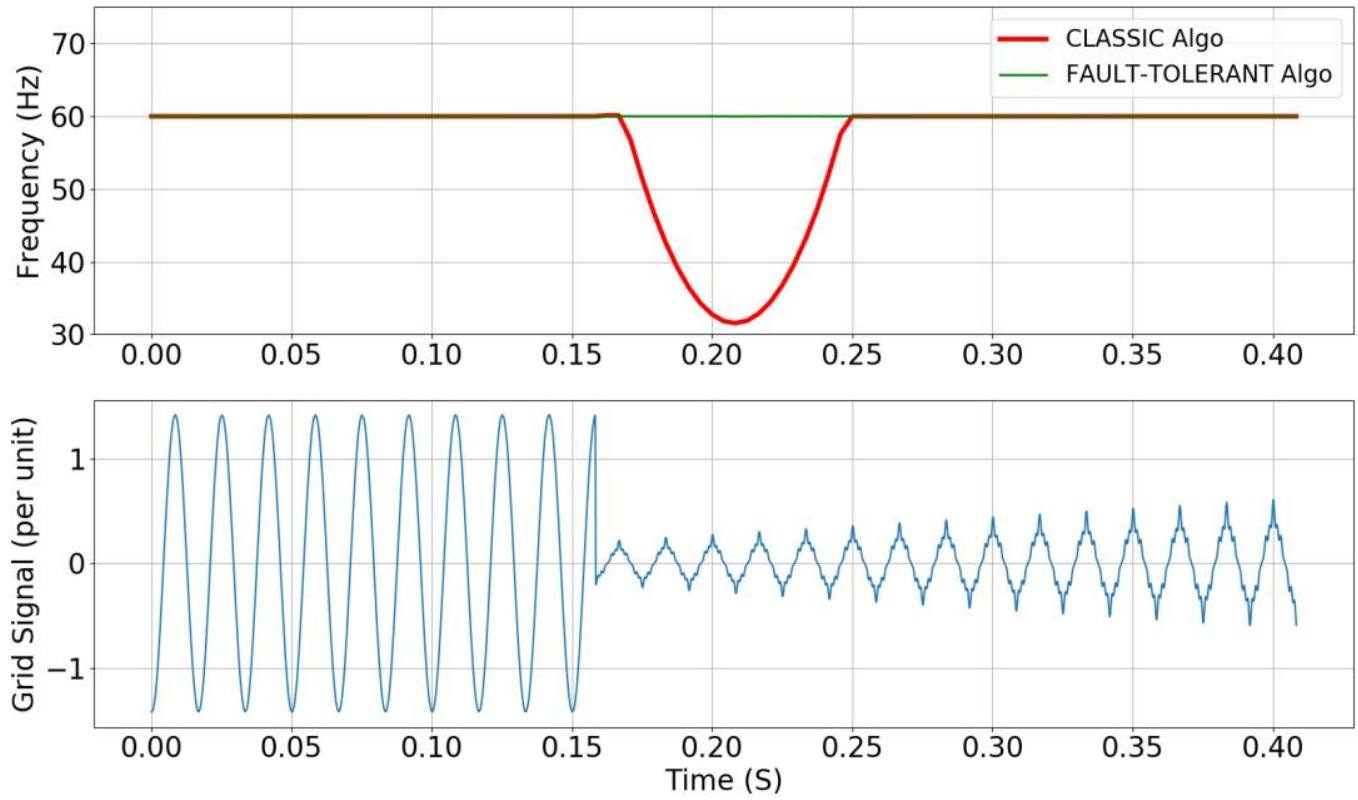

Figure 30 testing signal and results of case 9 


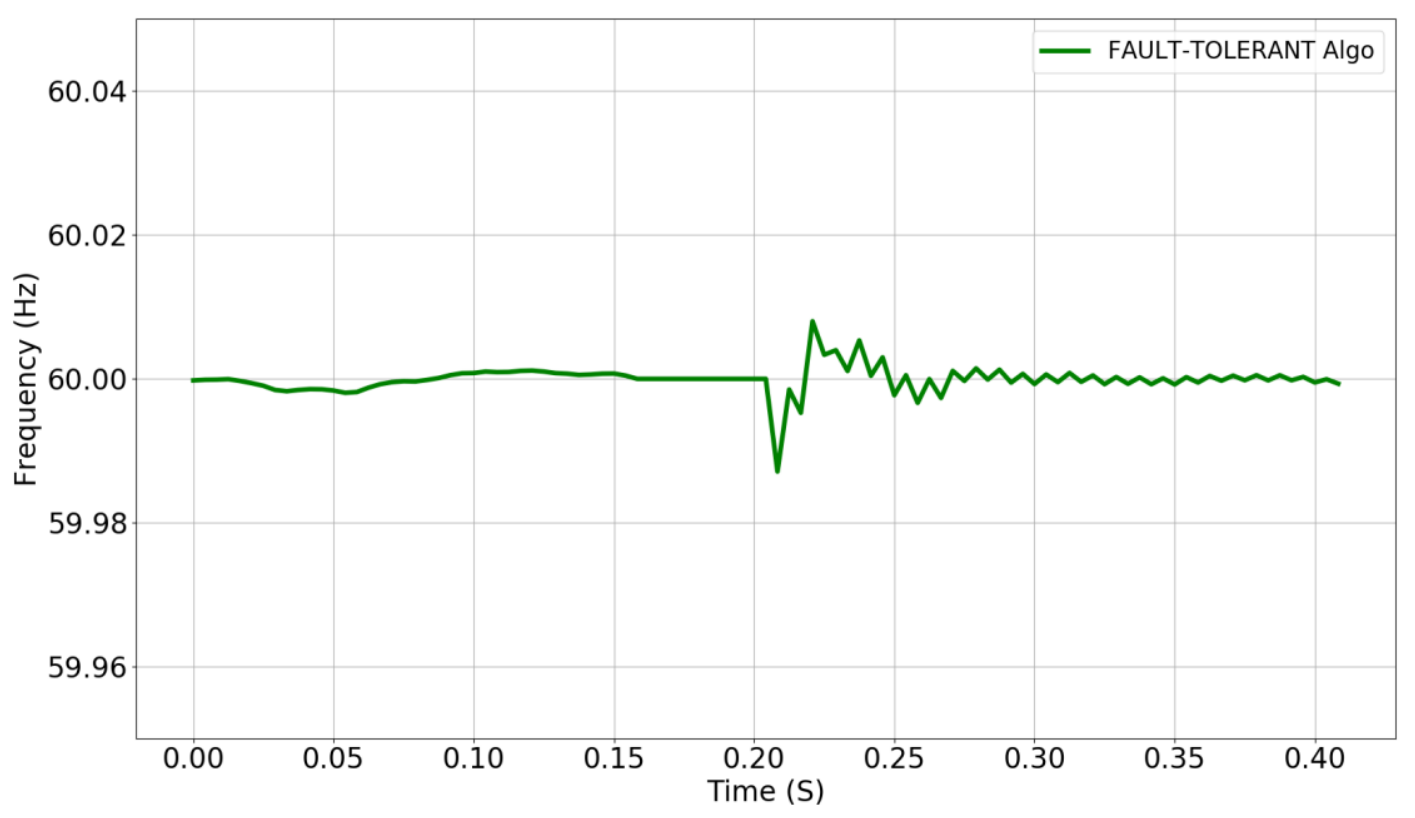

Figure 31 zoomed in estimated frequency by the fault-tolerant algorithm of case 9

We have also tested signals of different frequencies as listed in Table II, and results are presented below. The frequency measurement errors are within $0.5 \mathrm{~Hz}$ in two cycles and $0.05 \mathrm{~Hz}$ in 6 cycles following a phase-jump event, and all meet milestone 2 .
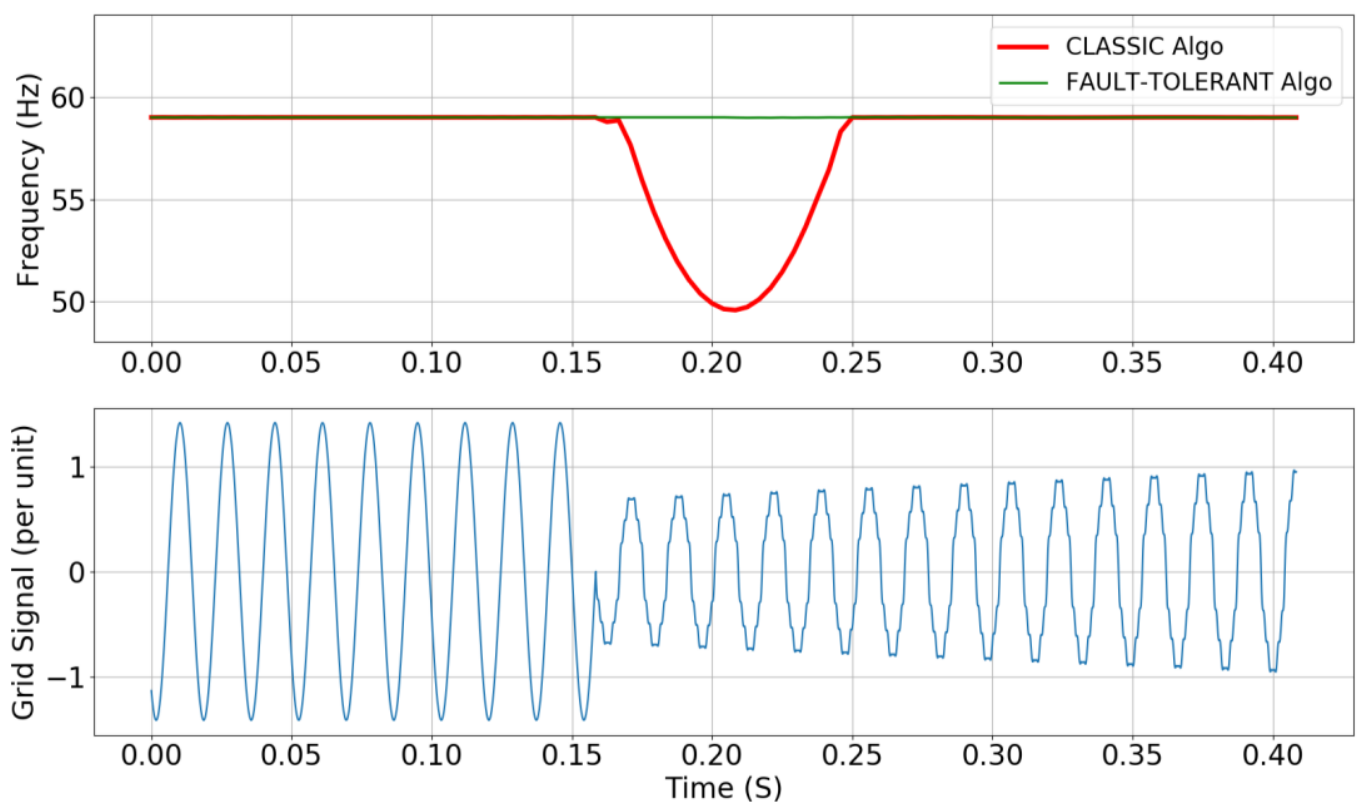

Figure 32 testing signal and results of case 10: $59 \mathrm{~Hz}$, base case 

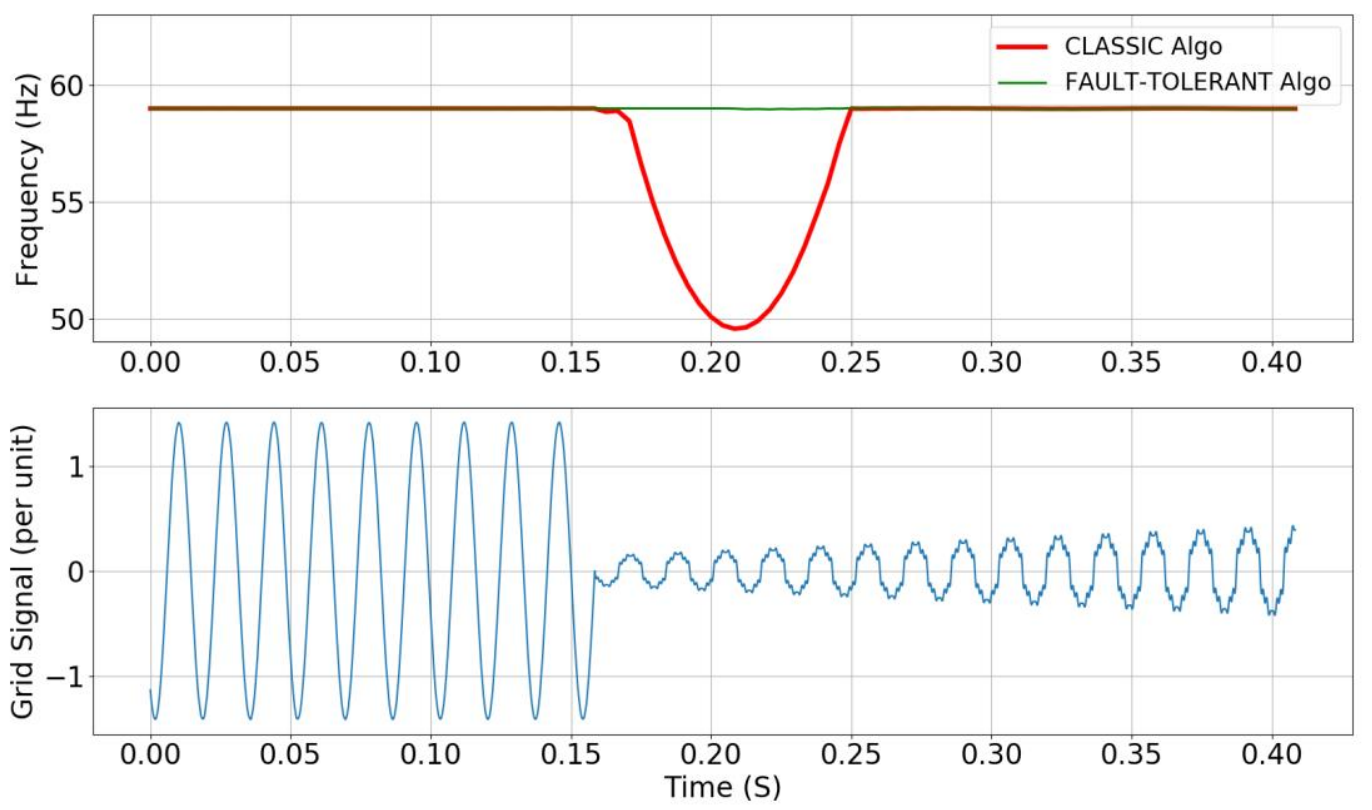

Figure 33 testing signal and results of case 11: $59 \mathrm{~Hz}$, worst case
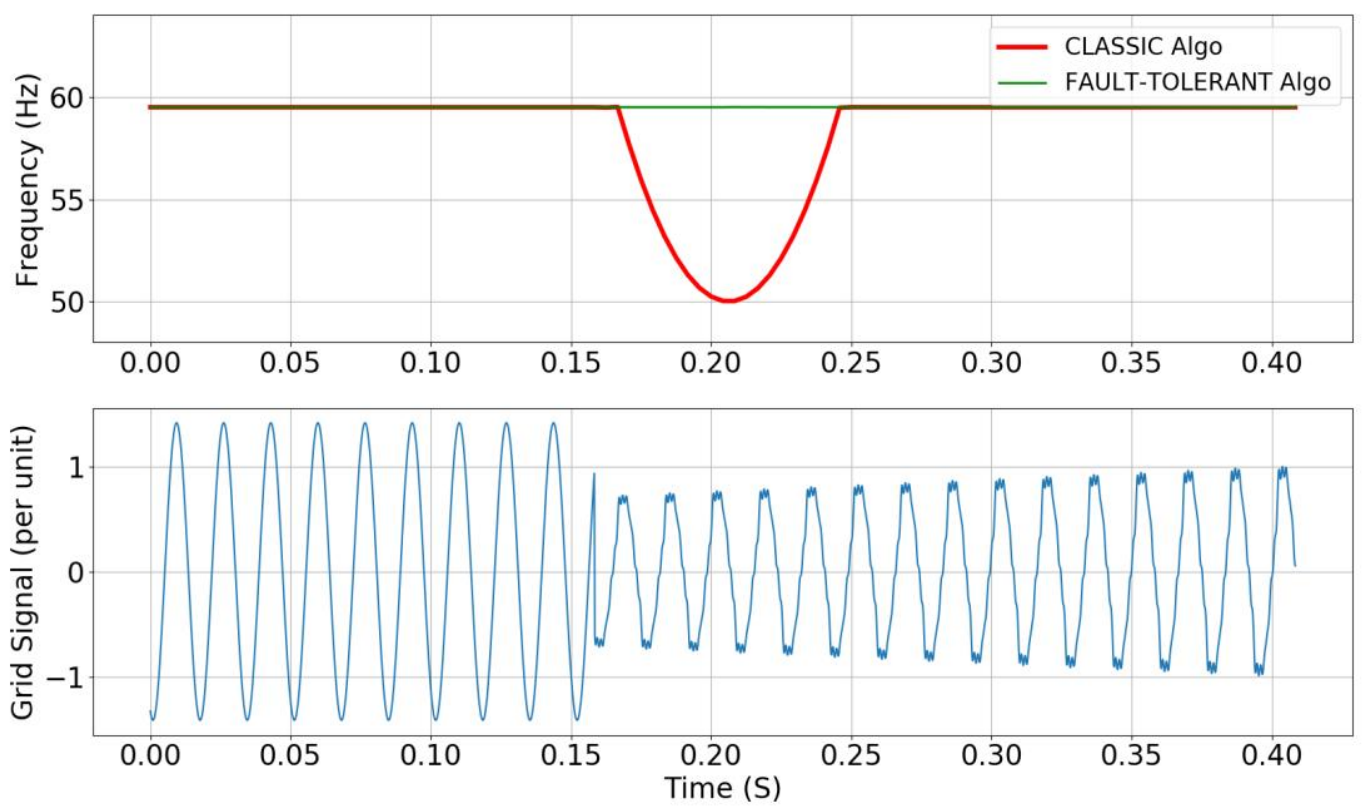

Figure 34 testing signal and results of case 12: $59.5 \mathrm{~Hz}$, base case 

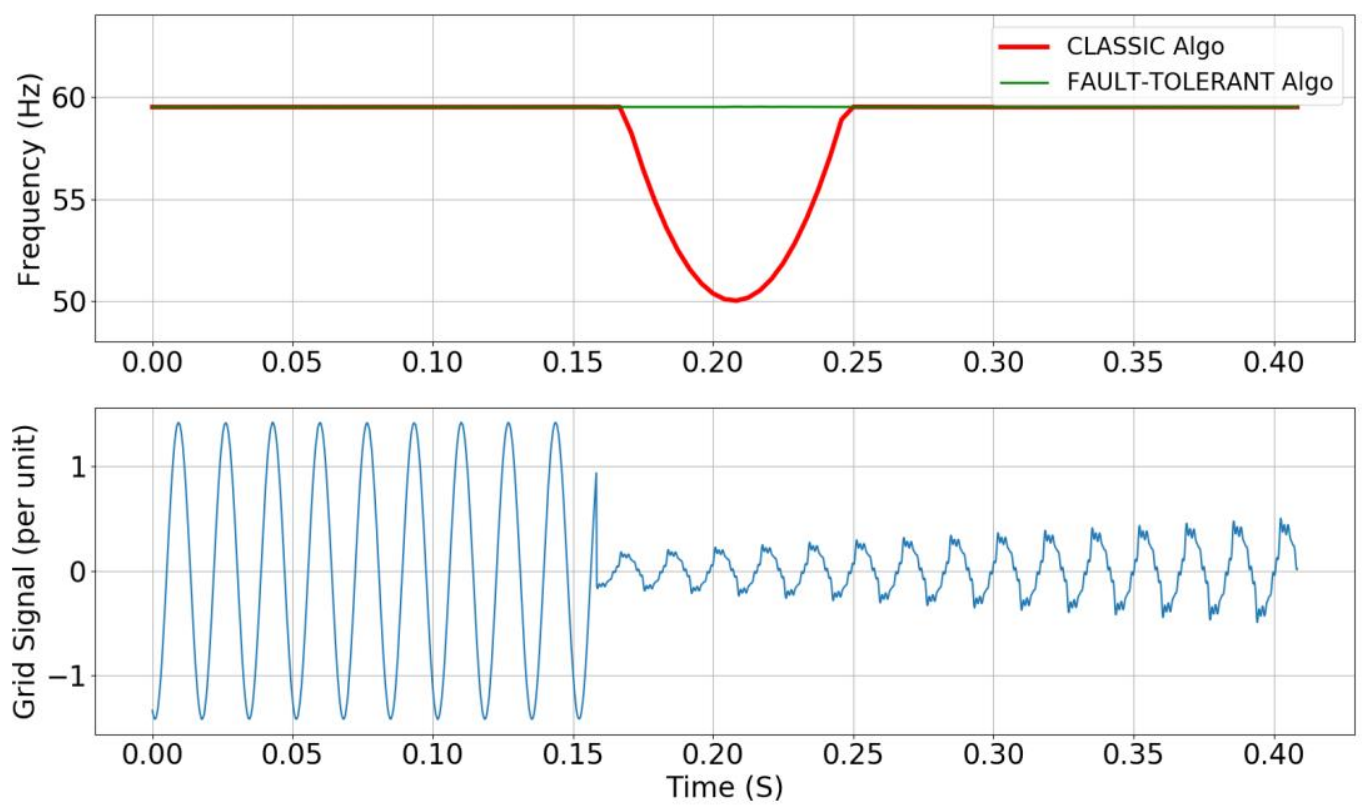

Figure 35 testing signal and results of case 13: $59.5 \mathrm{~Hz}$, worst case
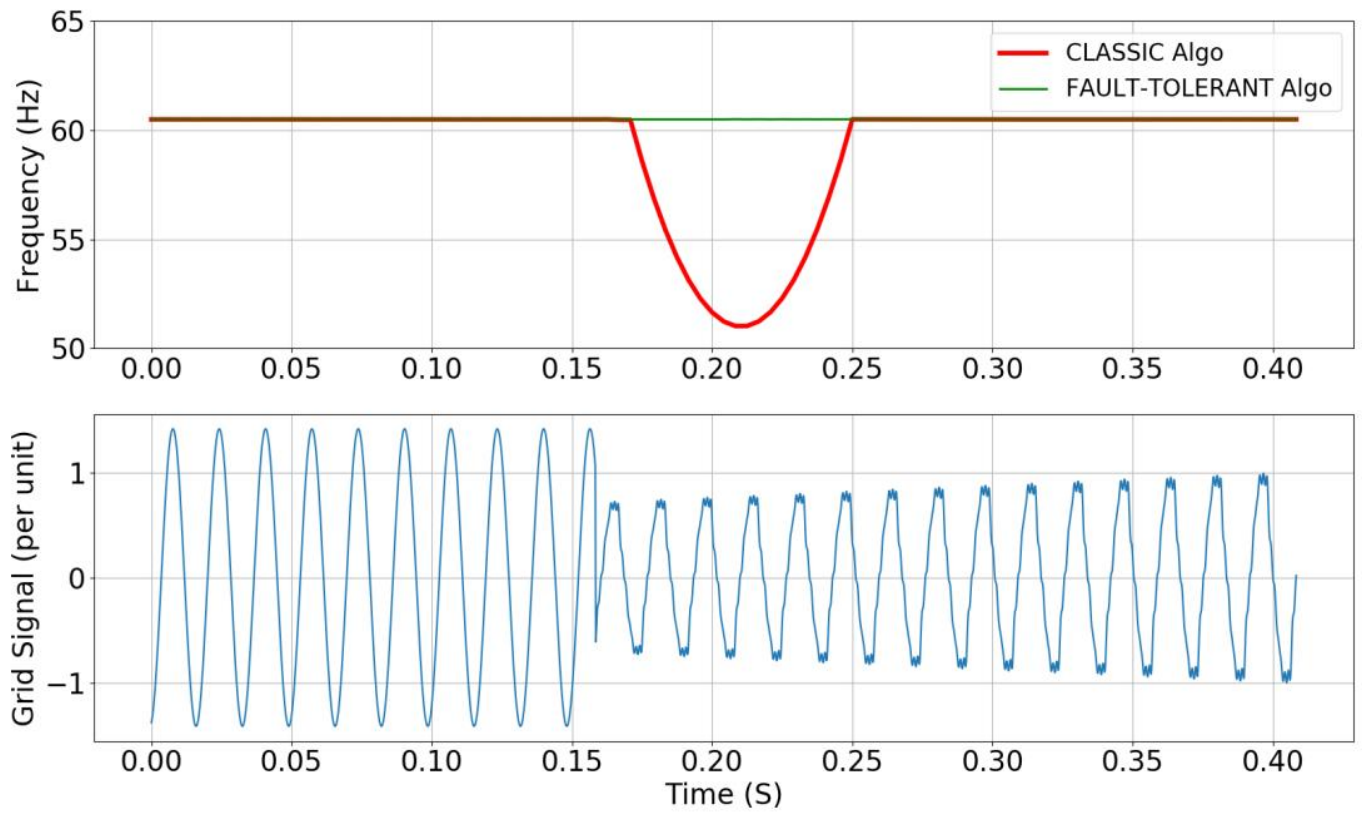

Figure 36 testing signal and results of case $14: 60.5 \mathrm{~Hz}$, base case 

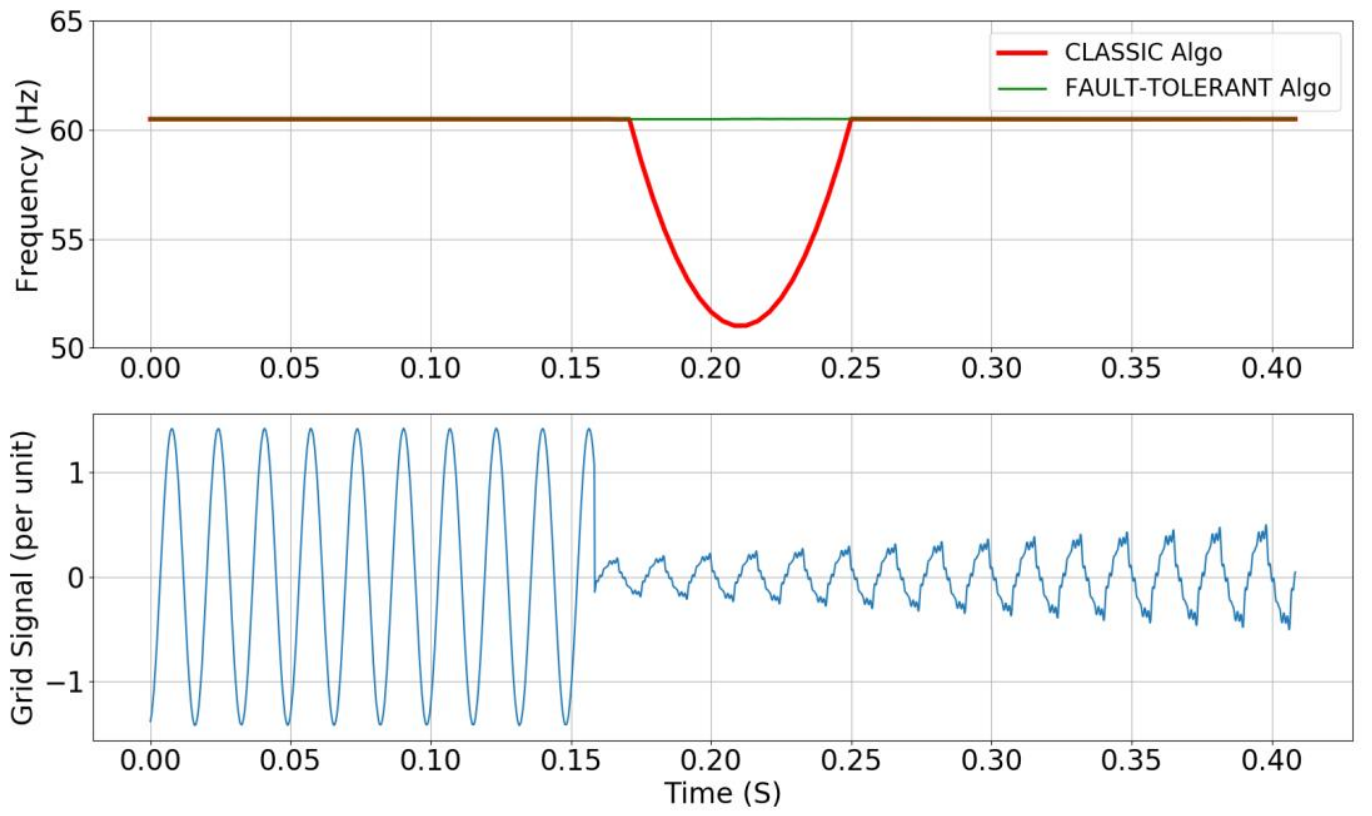

Figure 37 testing signal and results of case $15: 60.5 \mathrm{~Hz}$, worst case
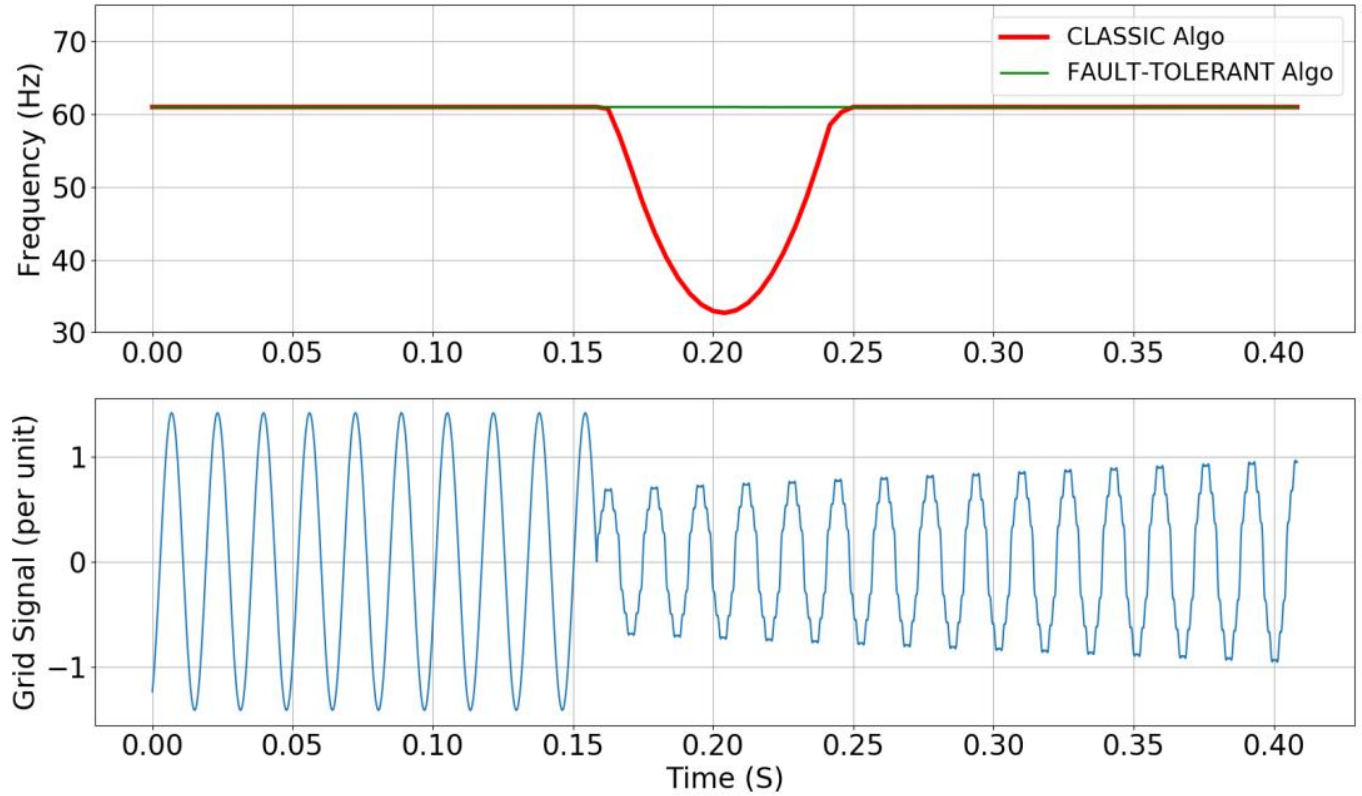

Figure 38 testing signal and results of case 16: $61 \mathrm{~Hz}$, base case 

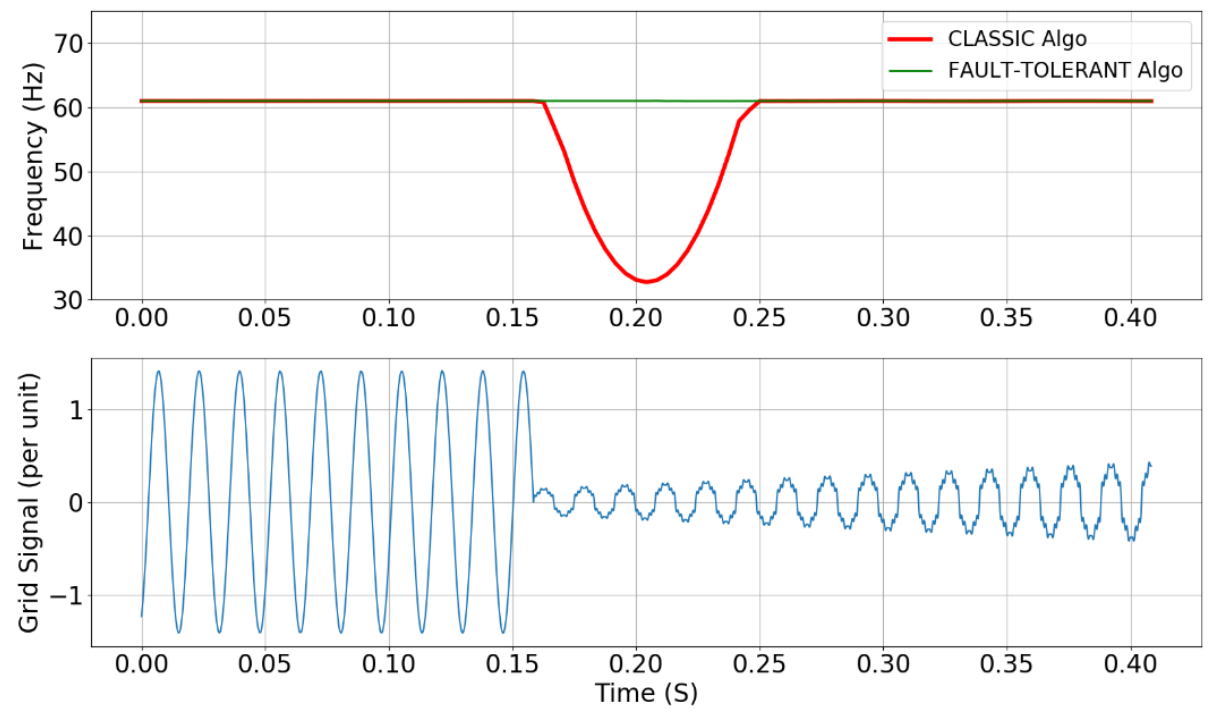

Figure 39 testing signal and results of case 17: $61 \mathrm{~Hz}$, worst case

\subsubsection{Accuracy and reliability evaluation, and further optimization of the developed algorithm}

In section 5.1.2 and 5.1.3, the fault-tolerant frequency estimator has been developed Preliminary testing has been performed to evaluate its performance. In this section, the team has performed a comprehensive testing. To be specific, as a fault-tolerant algorithm, it is critical that the algorithm has both high performance in the aspect of dependability and security. Dependability means that when a transient fault occurs and the fault can cause large measurement errors, the fault-tolerant algorithm shall be able to detect it and minimize the measurement errors. Security means that the algorithm shall not response to the signal distortions that will not introduce large measurement errors. The test performed in this section is focused on both dependability and security.

\subsubsection{Dependability test of fault-tolerant algorithm}

As mentioned, the dependability means the algorithm need to act when it shall act. In other words, the algorithm needs to be sensitive enough to detect a transient event and perform corresponding actions to mitigate measurement errors. In this test, the fault-tolerant frequency measurement algorithm has been tested with the fault waveforms with different levels of phase angle jump (i.e., from -60 degree to 60 degree). The phase jump waveforms are generated by applying phase-to-phase fault on the IEEE 30-Bus System and 118-Bus System, and the resultant waveforms contain not only phase jump but also voltage sag.

\section{IEEE system model and system setup}

Study has been performed on phase angle jump characterization associated with a fault, which has shown it mainly depends on transmission line impedance as well as the load [24]. By changing the length of transmission line, and hence the impedance, different degree of phase angle jump can be produced with a phase-to-phase fault. Theoretical analysis on a phase-to-phase fault is conducted first. Three-phase voltage can be expressed as:

$$
\left\{\begin{array}{l}
V_{a}=A * \operatorname{Sin}(2 \pi f t) \\
V_{b}=A * \operatorname{Sin}\left(2 \pi f t+120^{\circ}\right) \\
V_{c}=A * \operatorname{Sin}\left(2 \pi f t-120^{\circ}\right)
\end{array}\right.
$$


When a phase-to-phase fault happens to Phase A and B, assuming an ideal situation, the voltage near the fault location could be described as:

$$
\begin{gathered}
V_{a b}=A * \operatorname{Sin}(2 \pi f t)+A * \operatorname{Sin}\left(2 \pi f t+120^{\circ}\right) \\
V_{a b}=A * \operatorname{Sin}(2 \pi f t)+A * \operatorname{Sin}(2 \pi f t) \cos \left(120^{\circ}\right)+A * \cos (2 \pi f t) \sin \left(120^{\circ}\right)
\end{gathered}
$$

Subsequently, this can be further simplified as:

$$
V_{a b}=A * \operatorname{Sin}\left(2 \pi f t+60^{\circ}\right)
$$

It can be seen from above equations; the resultant phase angle jump in a phase-to-phase fault can reach $60^{\circ}$ in an ideal situation. Phase-A would lag $60^{\circ}$, whereas Phase-B would lead $60^{\circ}$. The resulted phase angle has a difference of $180^{\circ}$ from Phase-C. Considering the voltage drop due to the large current, the amplitude $A$ would have changed depending on the line impedance and the load.

To verify above equation, simulations have been conducted on the IEEE 118-bus system with PSCAD transient simulation software. Figure 40 partially displays the IEEE 118-bus system with a fault triggering module placed on Bus 60 .

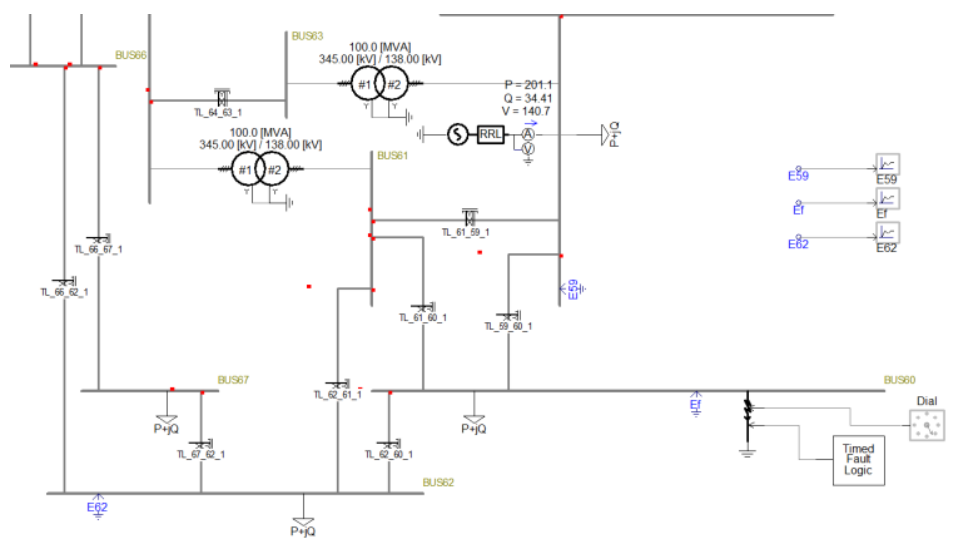

Figure 40 IEEE 118-bus system (partly shown) with line fault on Bus 60

Figure 41 shows the waveform when a phase-to-phase fault is triggered on Bus 60, and it can be observed that when the fault is triggered, the waveforms of Phase-A and Phase-B are almost overlapped with each other. By comparing with the reference waveforms without any fault, Phase-A lags $60^{\circ}$, and Phase-B leads $60^{\circ}$. The fault waveforms have $180^{\circ}$ phase difference from Phase-C and the amplitude is reduced by 50 percent. Thus, this result can be considered as a verification of the deduced equations.

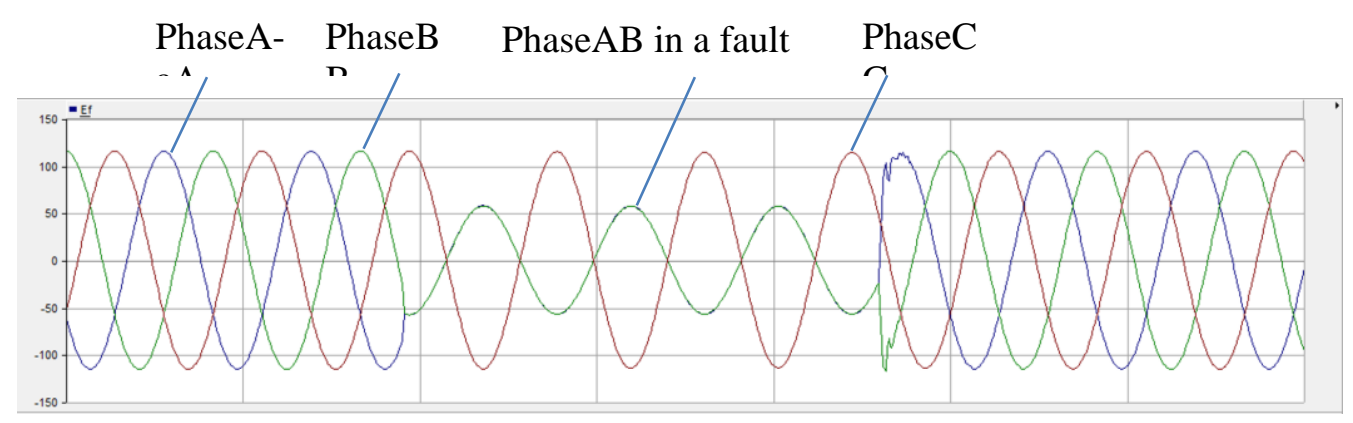

Figure 41 Voltage waveform of a phase-to-phase fault triggered at Bus 60 
A series of waveforms with different phase jump angles have been generated with IEEE system model, from -60 degree to 60 degree at a step of 10 degree. Figure 42 displays the waveforms with lagging (negative) angle jumps ranging from at $-10,-30,-50$ degree. The fault occurs at the peak of grid signal and lasts for $0.05 \mathrm{~s}$. Similarly, Figure 43 displays the waveforms with leading (positive) angle jumps ranging from at 10, 30, 50 degree.

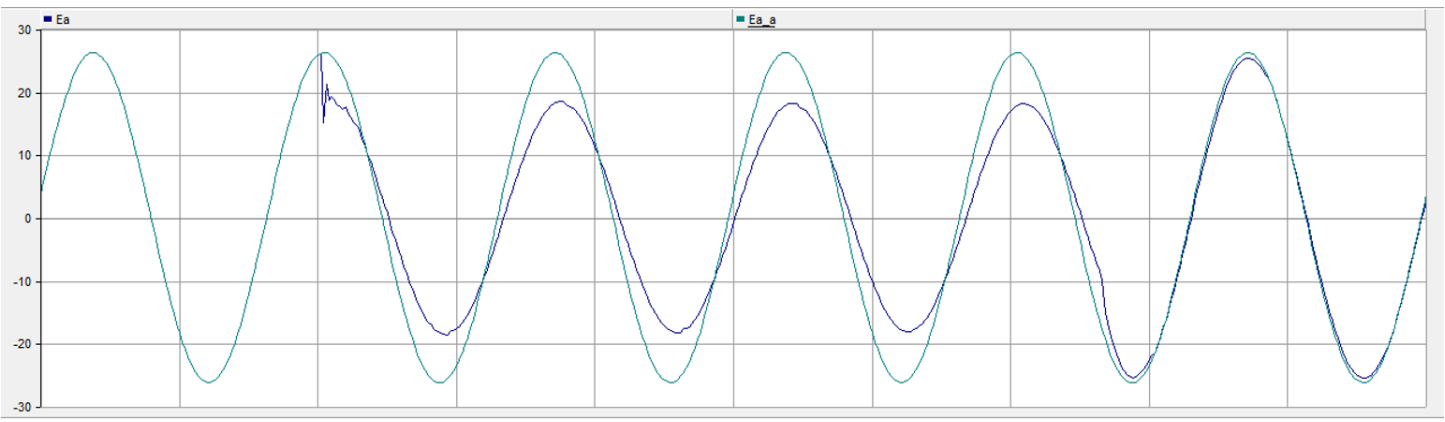

(a) Lagging 10

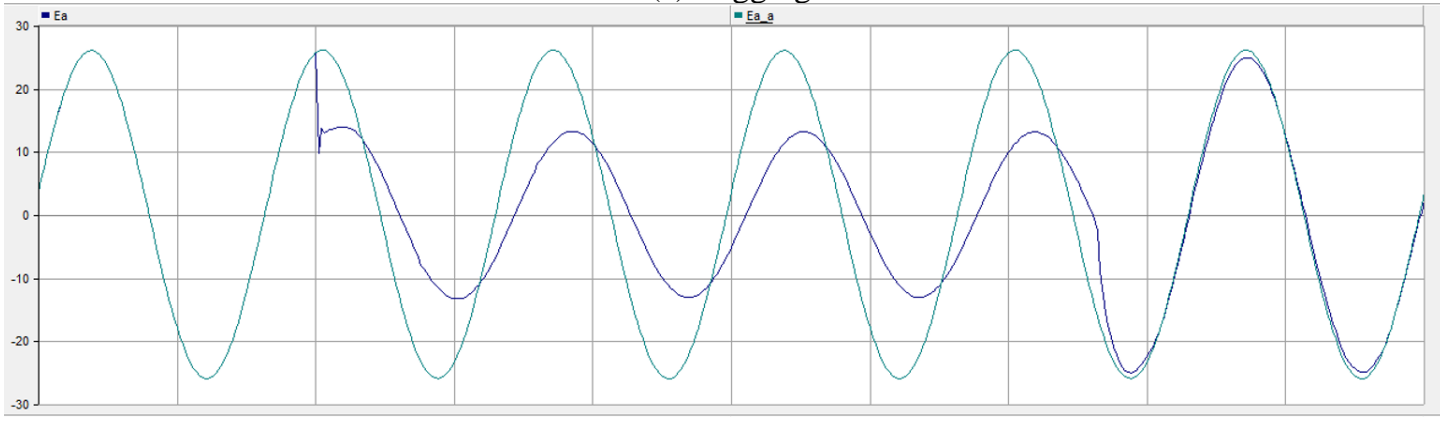

(b) Lagging $30^{\circ}$

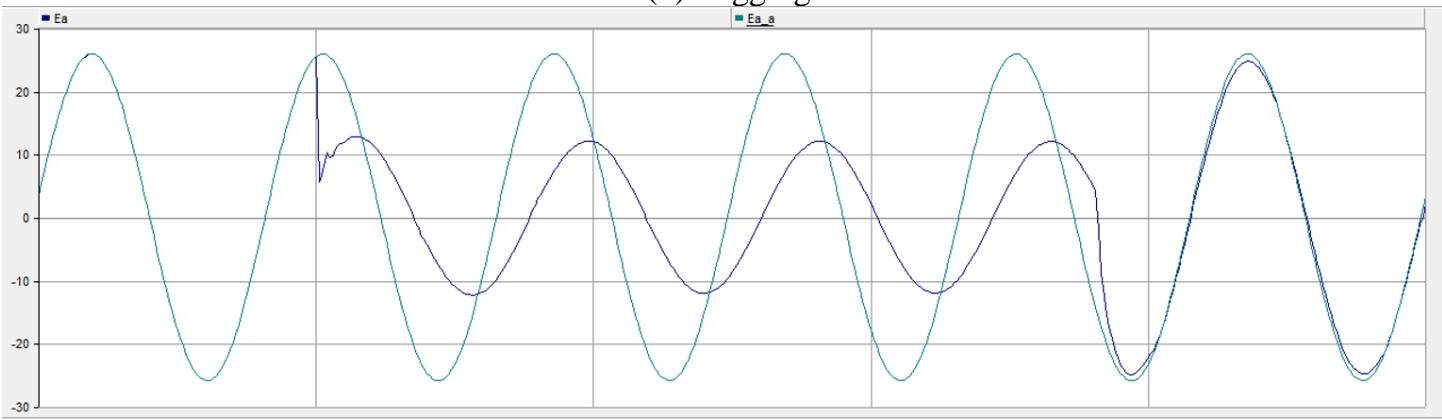

(c) Lagging $50^{\circ}$

Figure 42 Voltage waveforms with lagging angle jumps at $-10^{\circ},-30^{\circ},-50^{\circ}$ 


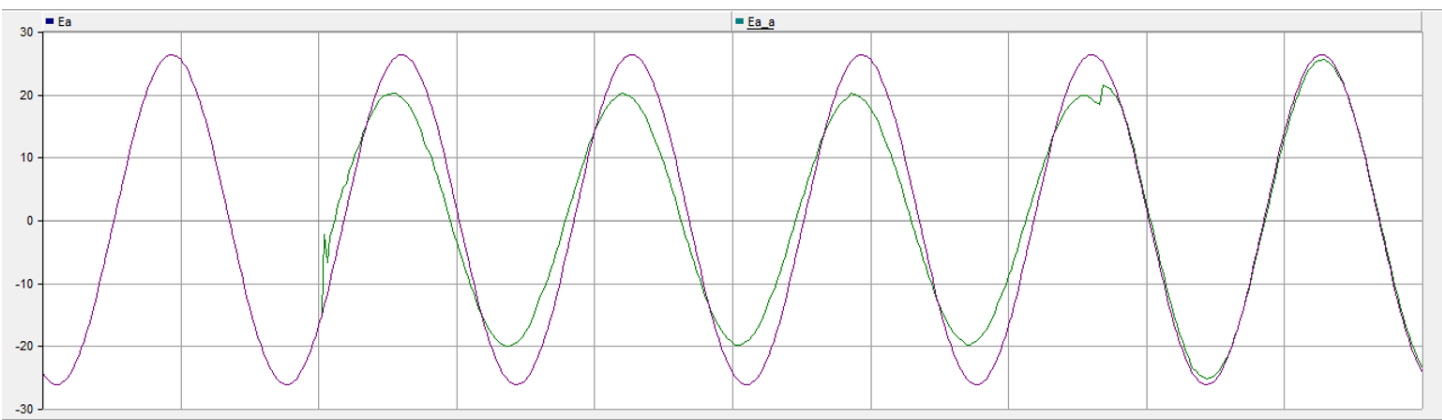

(a) Leading $10^{\circ}$

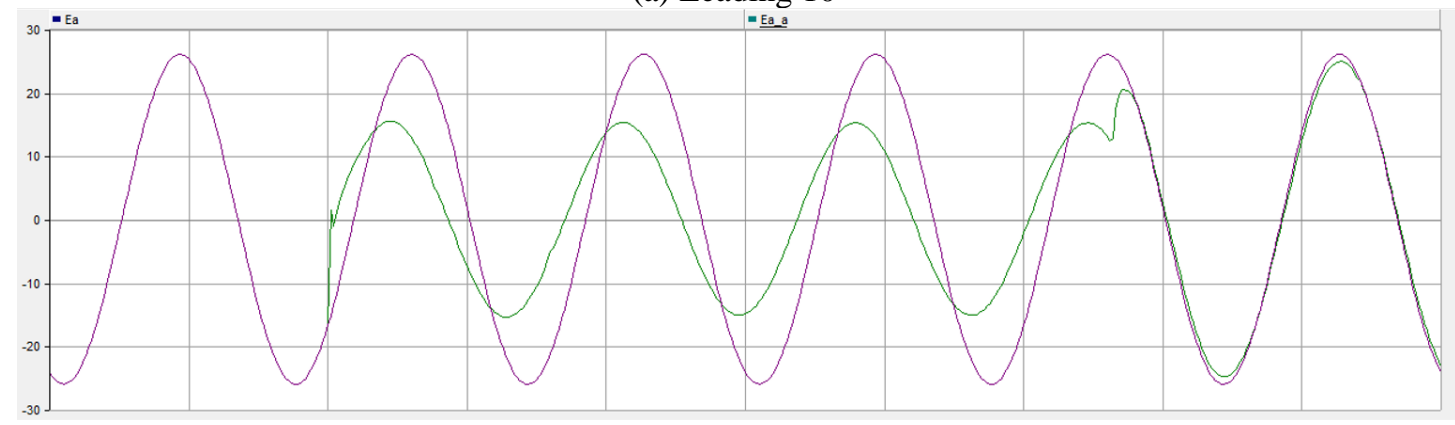

(b) Leading $30^{\circ}$

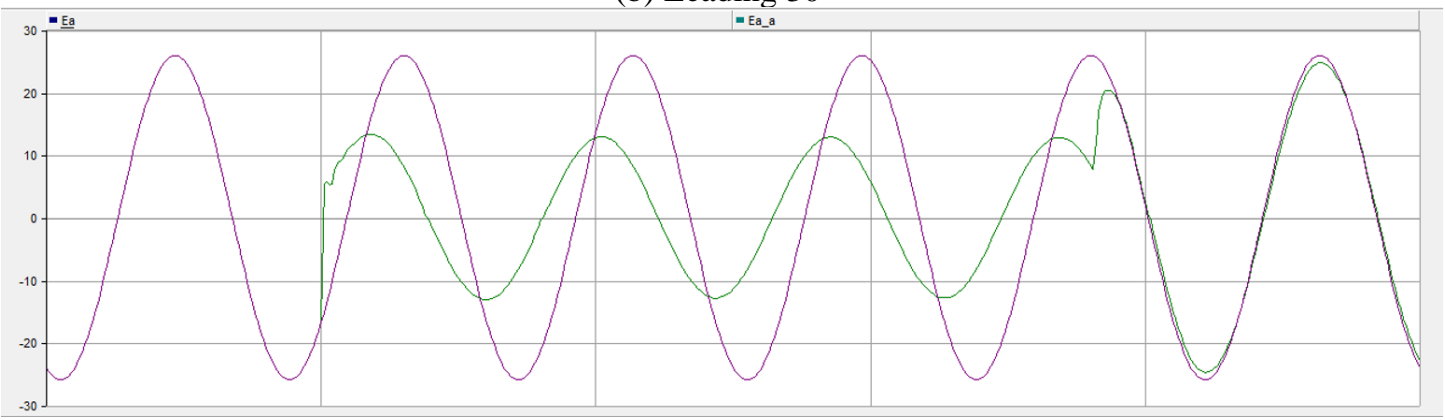

(c) Leading $50^{\circ}$

Figure 43 Voltage waveforms with leading angle jumps at $10^{\circ}, 30^{\circ}, 50^{\circ}$ 
For the fault-tolerant frequency measurement algorithm testing, these testing waveforms have been generated with angle jumps ranging from $-60^{\circ}$ to $+60^{\circ}$ in a step of $10^{\circ}$ (case 1-12). Additionally, instead of only applying the transient fault at the peak of grid signal, the transient fault was applied in different time of one fundamental frequency period, which are $1 / 4$ cycle after peak (case 13-25), $1 / 2$ cycle after peak (case 27-36), 3/4 cycle after peak (case 37-48). The detailed testing results are summarized from Table III to Table VI. In the tests, the normal system frequency is equal to $60 \mathrm{~Hz}$. During the system transient faults, the frequency measurement errors are calculated by:

$$
\text { Frequency errors }=\text { measured frequency }-60(\mathrm{~Hz})
$$

During system transients, the measured frequency can be higher and lower than $60 \mathrm{~Hz}$, therefore both the positive errors and negative errors are calculated and provided in each table.

Table III Measurement errors (Case 1 - 12)

\begin{tabular}{|c|c|c|c|c|}
\hline & \multicolumn{2}{|c|}{ Fault-tolerant Algorithm } & \multicolumn{2}{c|}{ Classical Algorithm } \\
\hline & Positive errors (Hz) & Negative errors (Hz) & Positive errors (Hz) & Negative errors (Hz) \\
\hline Case 1 & 0.123 & -0.032 & 0.577 & -16.048 \\
\hline Case 2 & 0.119 & -0.030 & 2.493 & -2.608 \\
\hline Case 3 & 0.116 & -0.023 & 2.007 & -2.116 \\
\hline Case 4 & 0.109 & -0.019 & 1.514 & -1.611 \\
\hline Case 5 & 0.094 & -0.019 & 1.009 & -1.089 \\
\hline Case 6 & 0.238 & -0.063 & 0.499 & -0.552 \\
\hline Case 7 & 0.124 & -0.034 & 0.563 & -23.960 \\
\hline Case 8 & 0.123 & -0.036 & 0.509 & -23.809 \\
\hline Case 9 & 0.119 & -0.035 & 0.451 & -16.965 \\
\hline Case 10 & 0.115 & -0.034 & 0.382 & -17.360 \\
\hline Case 11 & 0.106 & -0.047 & 1.208 & -1.288 \\
\hline Case 12 & 0.091 & -0.367 & 0.666 & -0.733 \\
\hline
\end{tabular}

Table IV Measurement errors (Case 13 - 24)

\begin{tabular}{|c|c|c|c|c|}
\hline & \multicolumn{2}{|c|}{ Fault-tolerant Algorithm } & \multicolumn{2}{c|}{ Classical Algorithm } \\
\hline & Positive errors (Hz) & $\begin{array}{c}\text { Negative errors } \\
(\mathrm{Hz})\end{array}$ & $\begin{array}{c}\text { Positive errors } \\
(\mathrm{Hz})\end{array}$ & $\begin{array}{c}\text { Negative errors } \\
(\mathrm{Hz})\end{array}$ \\
\hline Case 13 & 0.120 & -0.029 & 2.953 & -3.058 \\
\hline Case 14 & 0.118 & -0.029 & 2.487 & -2.590 \\
\hline Case 15 & 0.114 & -0.027 & 2.002 & -2.101 \\
\hline Case 16 & 0.111 & -0.022 & 1.509 & -1.600 \\
\hline Case 17 & 0.097 & -0.027 & 1.003 & -1.079 \\
\hline Case 18 & 0.272 & -0.069 & 0.496 & -0.544 \\
\hline Case 19 & 0.123 & -0.034 & 0.236 & -23.547 \\
\hline Case 20 & 0.121 & -0.031 & 0.212 & -23.433 \\
\hline Case 21 & 0.118 & -0.032 & 0.188 & -16.900 \\
\hline Case 22 & 0.113 & -0.032 & 0.163 & -17.305 \\
\hline Case 23 & 0.102 & -0.037 & 1.208 & -1.299 \\
\hline Case 24 & 0.101 & -0.402 & 0.664 & -0.741 \\
\hline
\end{tabular}


Table V Measurement errors (Case 25 - 36)

\begin{tabular}{|c|c|c|c|c|}
\hline & \multicolumn{2}{|c|}{ Fault-tolerant Algorithm } & \multicolumn{2}{c|}{ Classical Algorithm } \\
\hline & Positive errors (Hz) & $\begin{array}{c}\text { Negative errors } \\
(\mathrm{Hz})\end{array}$ & $\begin{array}{c}\text { Positive errors } \\
(\mathrm{Hz})\end{array}$ & $\begin{array}{c}\text { Negative errors } \\
(\mathrm{Hz})\end{array}$ \\
\hline Case 25 & 0.123 & -0.032 & 2.958 & -3.078 \\
\hline Case 26 & 0.119 & -0.031 & 2.493 & -2.608 \\
\hline Case 27 & 0.116 & -0.023 & 2.007 & -2.116 \\
\hline Case 28 & 0.109 & -0.019 & 1.514 & -1.612 \\
\hline Case 29 & 0.095 & -0.019 & 1.009 & -1.089 \\
\hline Case 30 & 0.238 & -0.063 & 0.499 & -0.552 \\
\hline Case 31 & 0.124 & -0.034 & 0.104 & -16.136 \\
\hline Case 32 & 0.123 & -0.036 & 2.452 & -2.547 \\
\hline Case 33 & 0.119 & -0.035 & 2.054 & -21.093 \\
\hline Case 34 & 0.115 & -0.034 & 1.651 & -20.686 \\
\hline Case 35 & 0.106 & -0.047 & 1.208 & -1.288 \\
\hline Case 36 & 0.091 & -0.368 & 0.666 & -0.733 \\
\hline
\end{tabular}

Table VI Measurement errors (Case 37 - 48)

\begin{tabular}{|c|c|c|c|c|}
\hline & \multicolumn{2}{|c|}{ Fault-tolerant Algorithm } & \multicolumn{2}{c|}{ Classical Algorithm } \\
\hline & Positive errors (Hz) & $\begin{array}{c}\text { Negative errors } \\
(\mathrm{Hz})\end{array}$ & $\begin{array}{c}\text { Positive errors } \\
(\mathrm{Hz})\end{array}$ & $\begin{array}{c}\text { Negative errors } \\
(\mathrm{Hz})\end{array}$ \\
\hline Case 37 & 0.120 & -0.029 & 2.953 & -21.988 \\
\hline Case 38 & 0.118 & -0.029 & 2.487 & -21.520 \\
\hline Case 39 & 0.114 & -0.027 & 2.002 & -2.101 \\
\hline Case 40 & 0.111 & -0.022 & 1.509 & -1.600 \\
\hline Case 41 & 0.097 & -0.027 & 1.003 & -1.079 \\
\hline Case 42 & 0.272 & -0.069 & 0.496 & -0.544 \\
\hline Case 43 & 0.123 & -0.034 & 0.611 & -16.442 \\
\hline Case 44 & 0.121 & -0.031 & 0.524 & -16.739 \\
\hline Case 45 & 0.118 & -0.032 & 0.446 & -29.647 \\
\hline Case 46 & 0.112 & -0.032 & 1.654 & -20.698 \\
\hline Case 47 & 0.102 & -0.037 & 1.208 & -1.299 \\
\hline Case 48 & 0.101 & -0.402 & 0.664 & -0.741 \\
\hline
\end{tabular}

Further, the data are also plotted in Figure 44 to Figure 47. In each figure, the green bar represents the measurement errors of the fault-tolerant algorithm while the blue bar represents the measurement errors of the classical algorithm. Each bar ranges from negative to positive. In each plot, two red threshold lines at 1 and -3 are plotted as $61 \mathrm{~Hz}$ is the upper limit of trigger frequency while $57 \mathrm{~Hz}$ is the lower limit of trigger frequency. As we can observe from the four figures, the measurement errors of the fault-tolerant algorithm are far less than the errors of the classical algorithm. More importantly, we can see the errors of the fault-tolerant algorithm are all below the thresholds (i.e., $1 \mathrm{~Hz}$, and $-3 \mathrm{~Hz}$ ) while for most of cases, the measurement errors of the classical algorithm are over the thresholds, and in about $50 \%$ cases, the measurement errors of the classical algorithm are far over the thresholds. In other words, if the measurement errors are greater than any of the two thresholds (1 or -3), it could cause mis-trip of DERs, and it was exactly what happened in the blue cut fire event on Aug. 2016 (700 MW utility scale solar falsely tripped due to incorrect frequency measurements). With the help of the fault-tolerant algorithm, the similar events can be prevented due to its robust measurement performance under system transient faults. 


\section{Milestone \#3}

In this project, our Milestone \# 3 is "prevention of false tripping by the developed algorithm at a success rate of over $90 \%$ by applying transient faults using IEEE benchmark system model."

Among the 48 tests we performed, the number of cases whose frequency measurement errors by the classical algorithm are greater than $1 \mathrm{~Hz}$ or lower than $-3 \mathrm{~Hz}$ is 40 while the number of cases whose frequency measurement errors by the fault-tolerant algorithm are greater than $1 \mathrm{~Hz}$ or lower than $-3 \mathrm{~Hz}$ is 0 . Therefore, the success rate of fault-tolerant algorithm is calculated as follows:

$$
r_{\text {success }}=\frac{40}{40}=100 \%
$$

The success rate is $100 \%$ and meets the $90 \%$ success rate in milestone \#3.

\begin{tabular}{|l|l|l|l|}
\hline Milestone & Metric Definition & $\begin{array}{l}\text { Finished } \\
\text { Date }\end{array}$ & $\begin{array}{l}\text { Success } \\
\text { Value }\end{array}$ \\
\hline 3 & $\begin{array}{l}\text { prevention of false tripping by the developed algorithm at a success } \\
\text { rate of over 90\% by applying transient faults using IEEE } \\
\text { benchmark system model. }\end{array}$ & $6 / 30 / 2019$ & $\mathbf{1 0 0 \%}$ \\
\hline
\end{tabular}

Without the fault-tolerant algorithm, the false trip rate of the classical algorithm is $40 / 48=83.33 \%$ while the false trip rate of the fault-tolerant algorithm is $0 / 48=0 \%$. Therefore, we can conclude that the fault-tolerant algorithm performs very well under system transients and can contribute to prevent false trip of DERs due to incorrect frequency measurements.

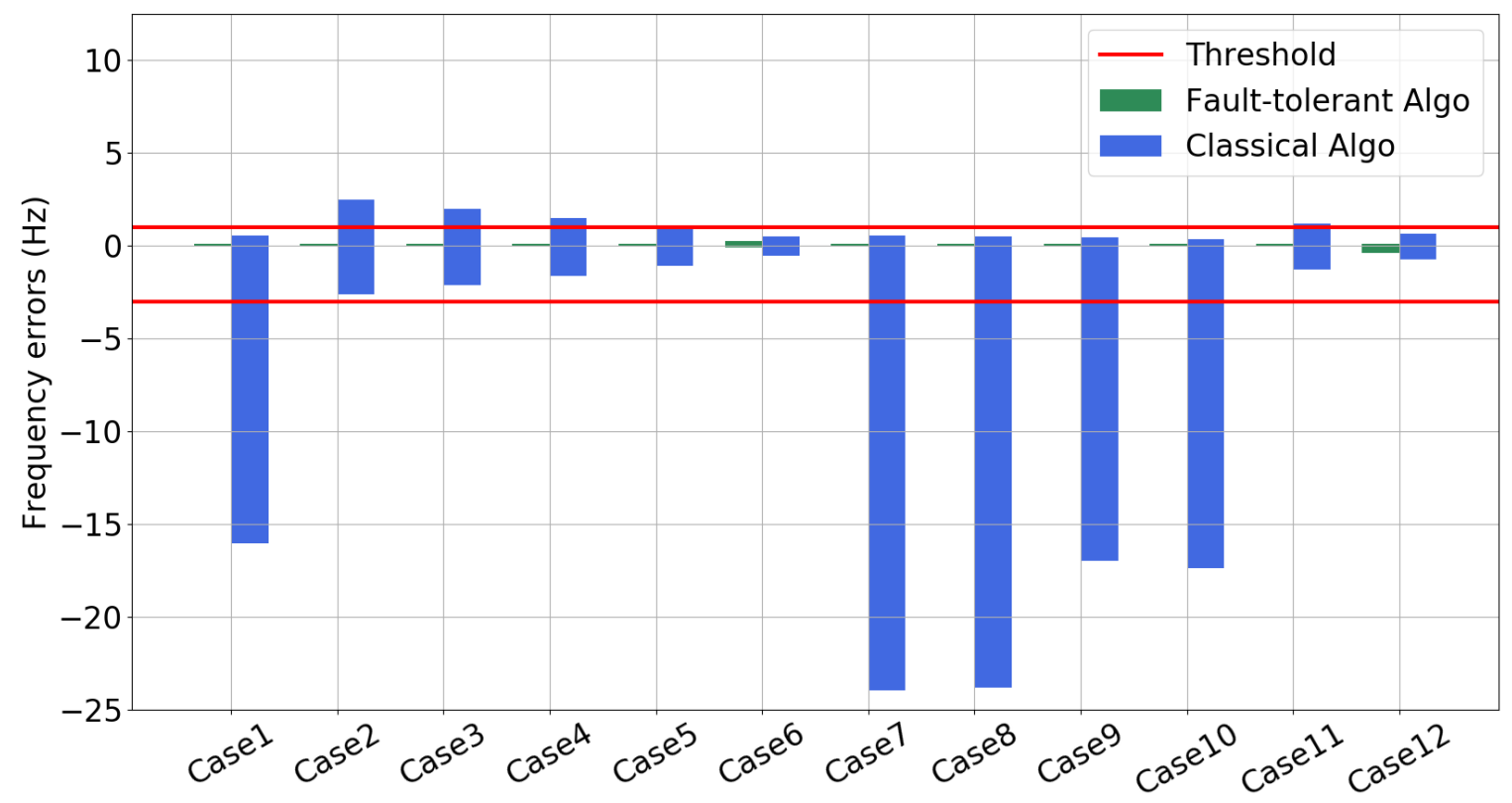

Figure 44 IEEE system model case 1 - 12 


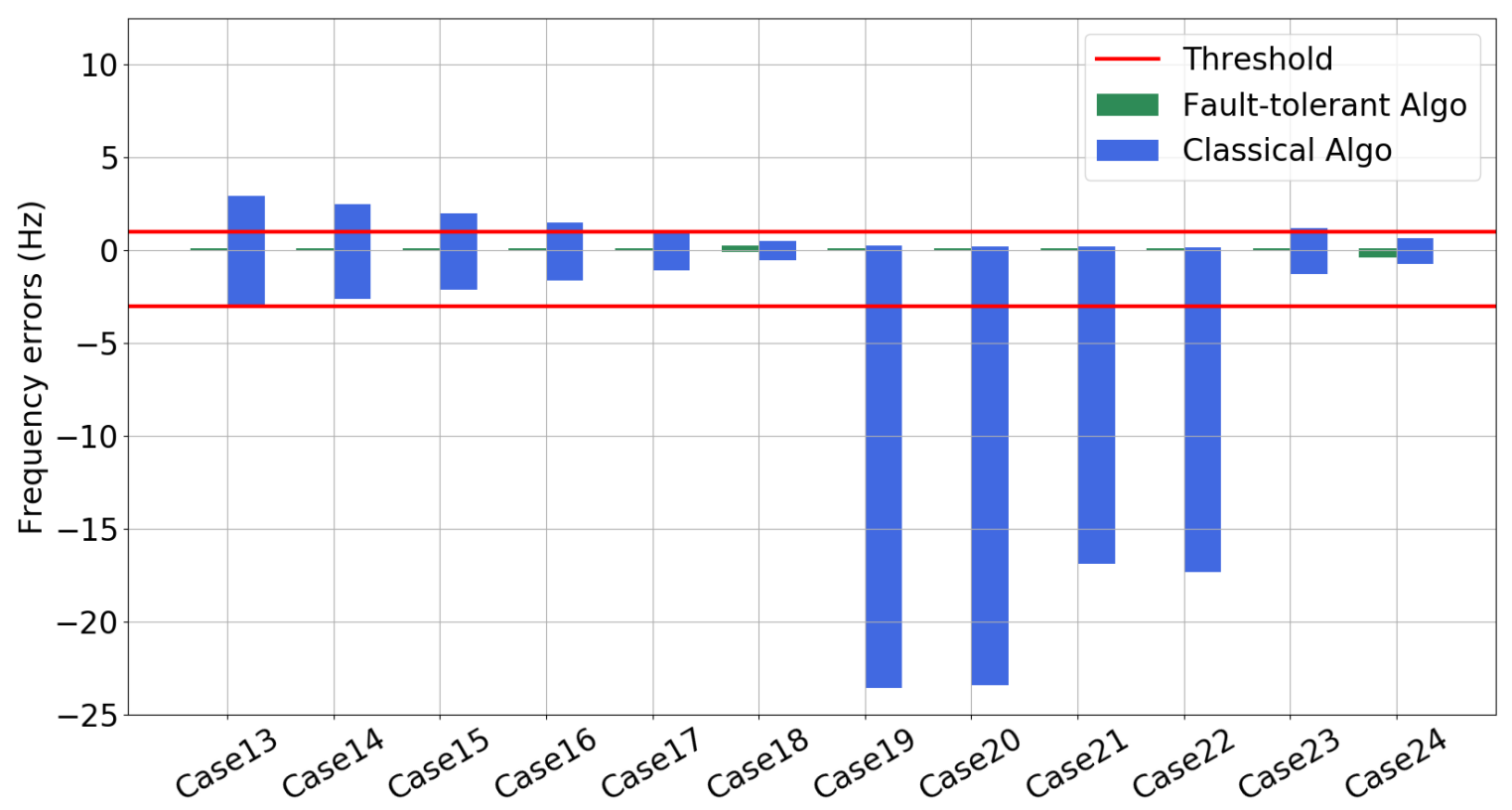

Figure 45 IEEE system model case 13 - 24

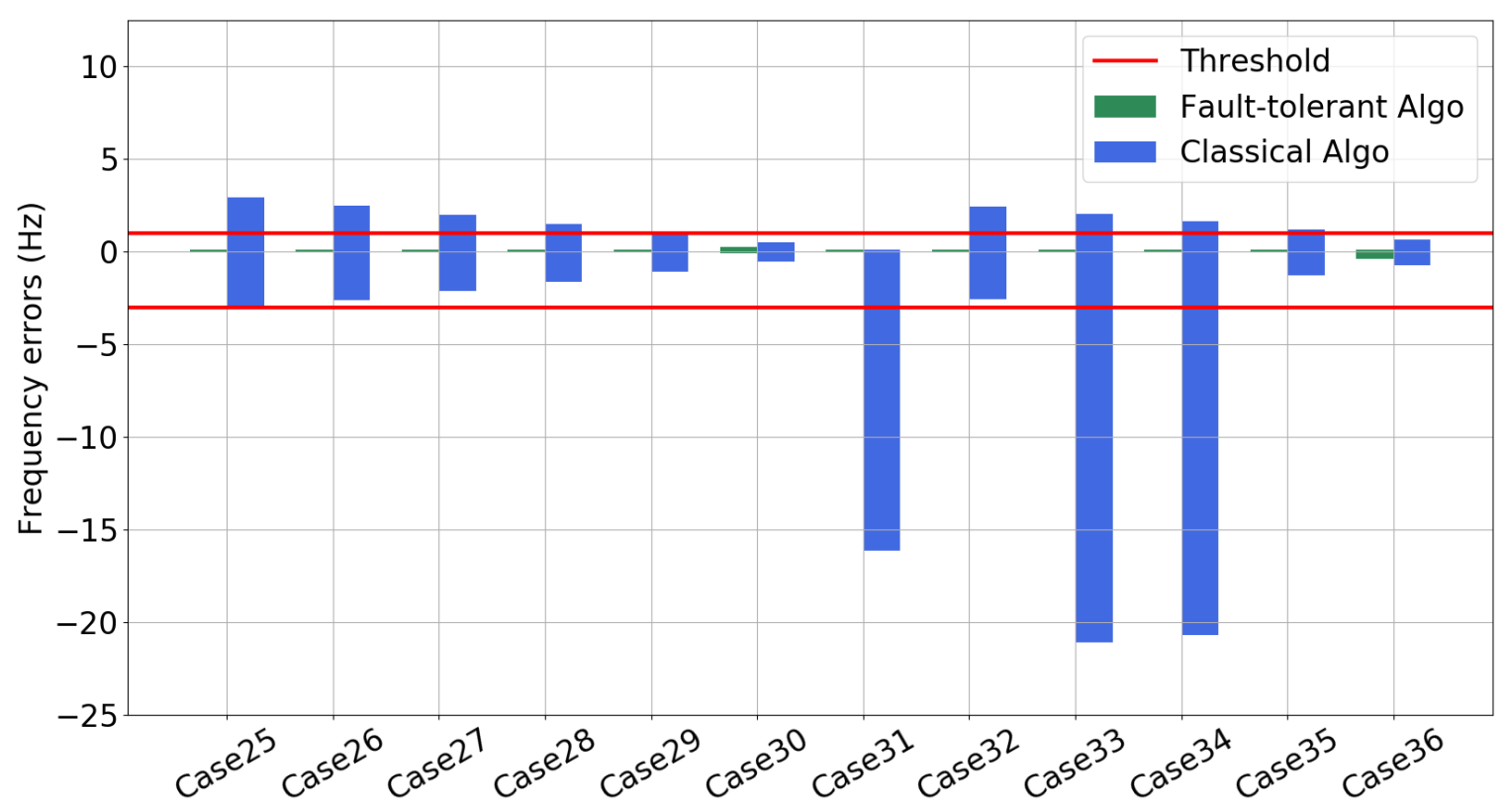

Figure 46 IEEE system model case 25 - 36 


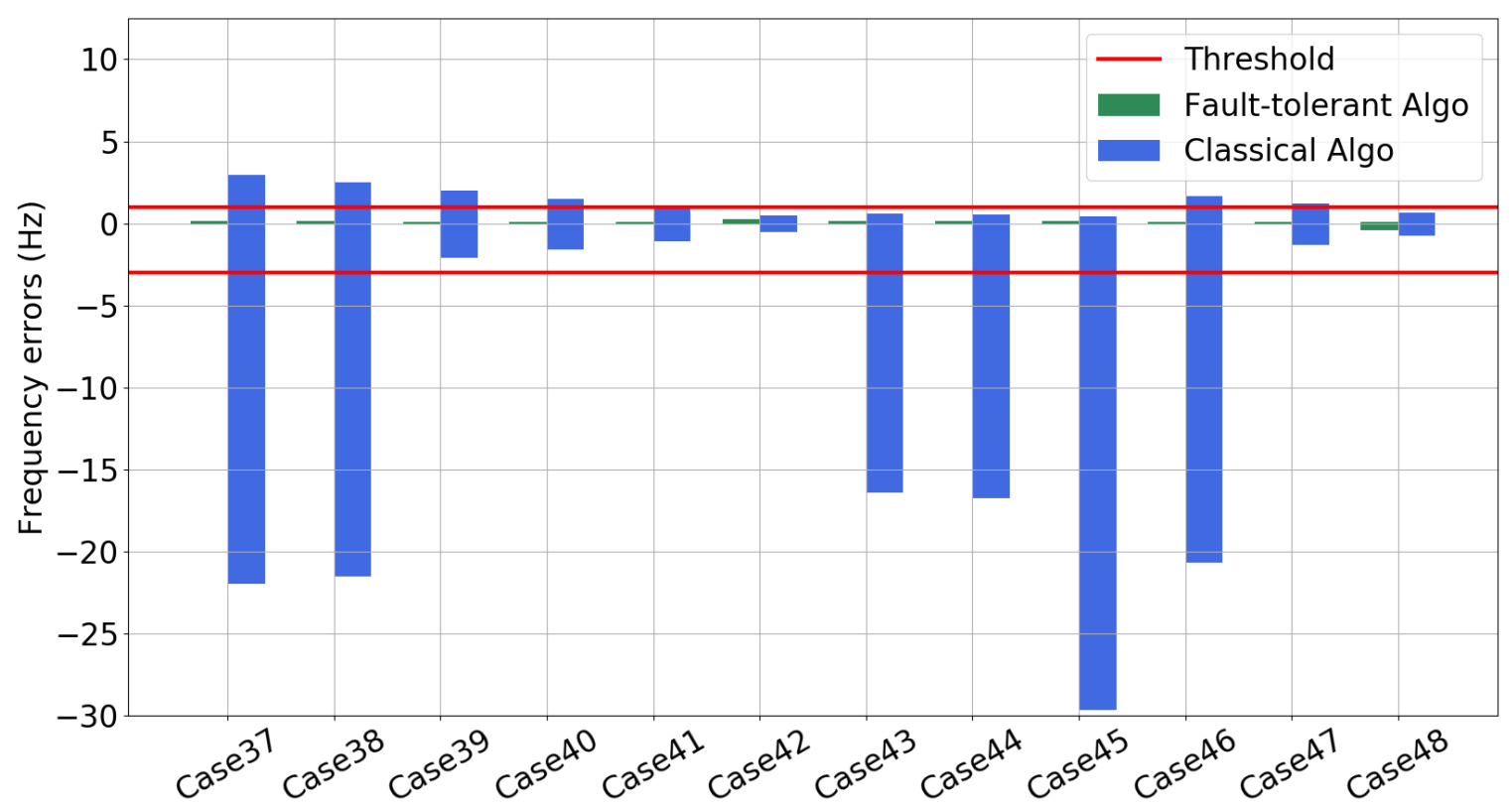

Figure 47 IEEE system model case 37 - 48 
The time domain testing results of case 1, 13, 25, and 37 are shown in Figure 48 to Figure 51, and all other cases are provided in Appendix A. The blue and green waveforms represent the frequency measurements of classical algorithm and fault-tolerant algorithm, respectively. The fault-tolerant measurement algorithm has significantly less measurement errors during the transients. The black waveform represents the raw and point-on-wave grid signal with a fault occurring at about 0.2 second and cleared about 3 cycles later. The red waveforms represent the system transient detection ( 0 indicates no transient detected, and 1 indicates a transient detected), and we can see that the transient detector successfully detected the system transient when the fault occurs and clears.
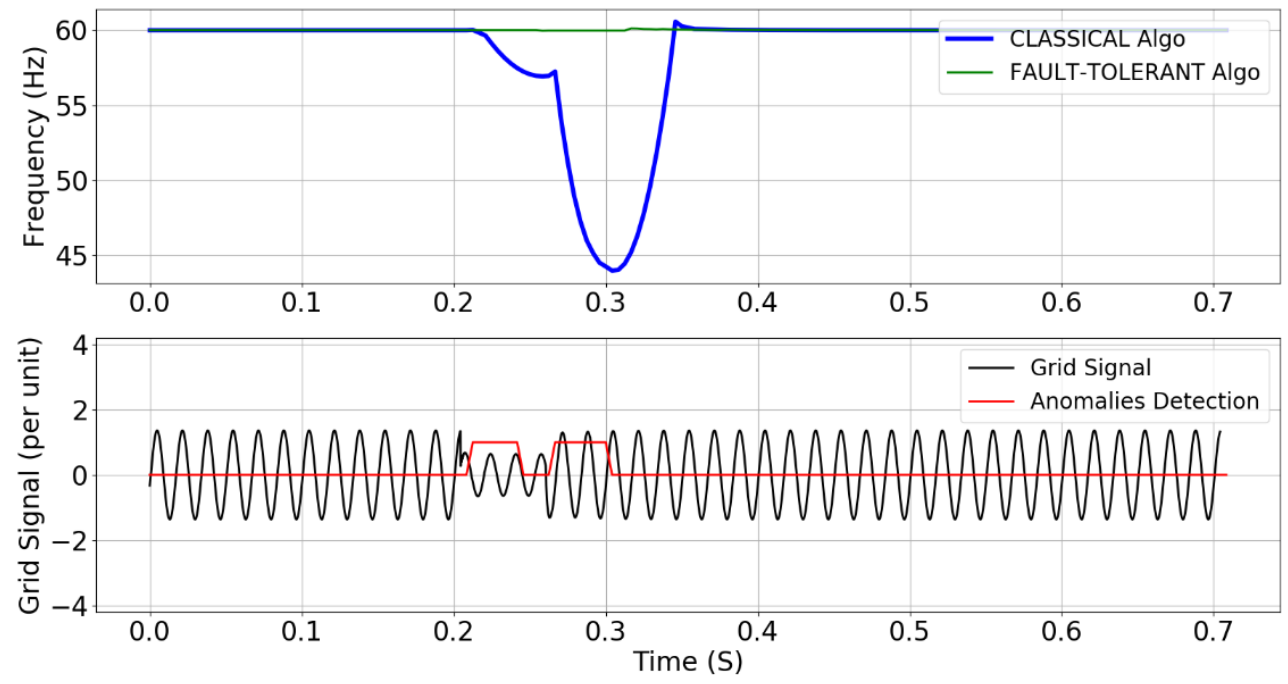

Figure 48 time domain result - case 1
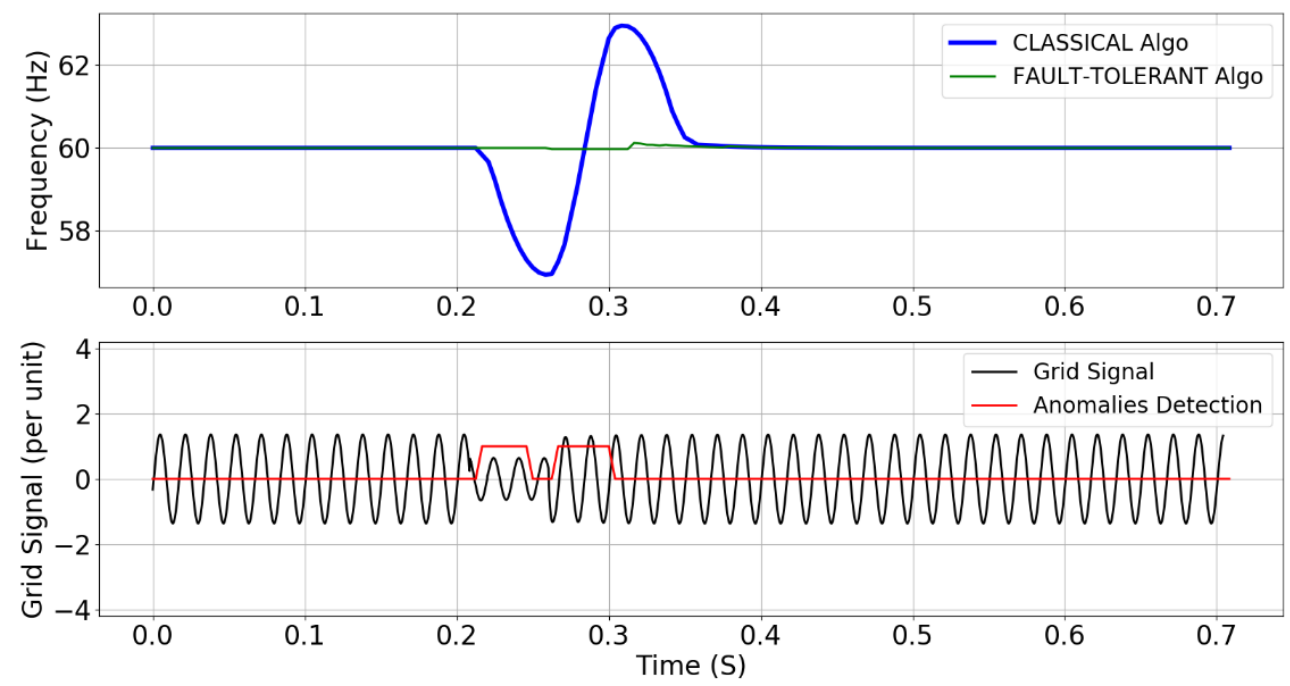

Figure 49 time domain result - case 13 

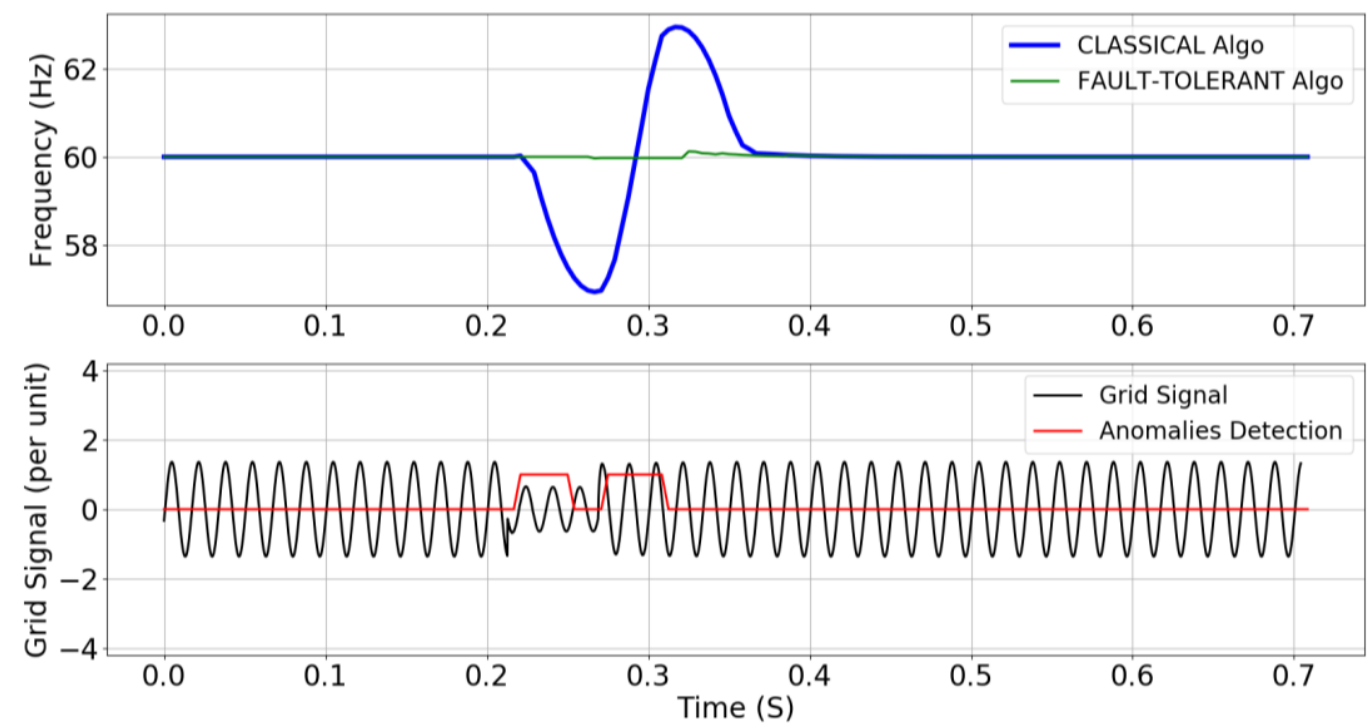

Figure 50 time domain result - case 25
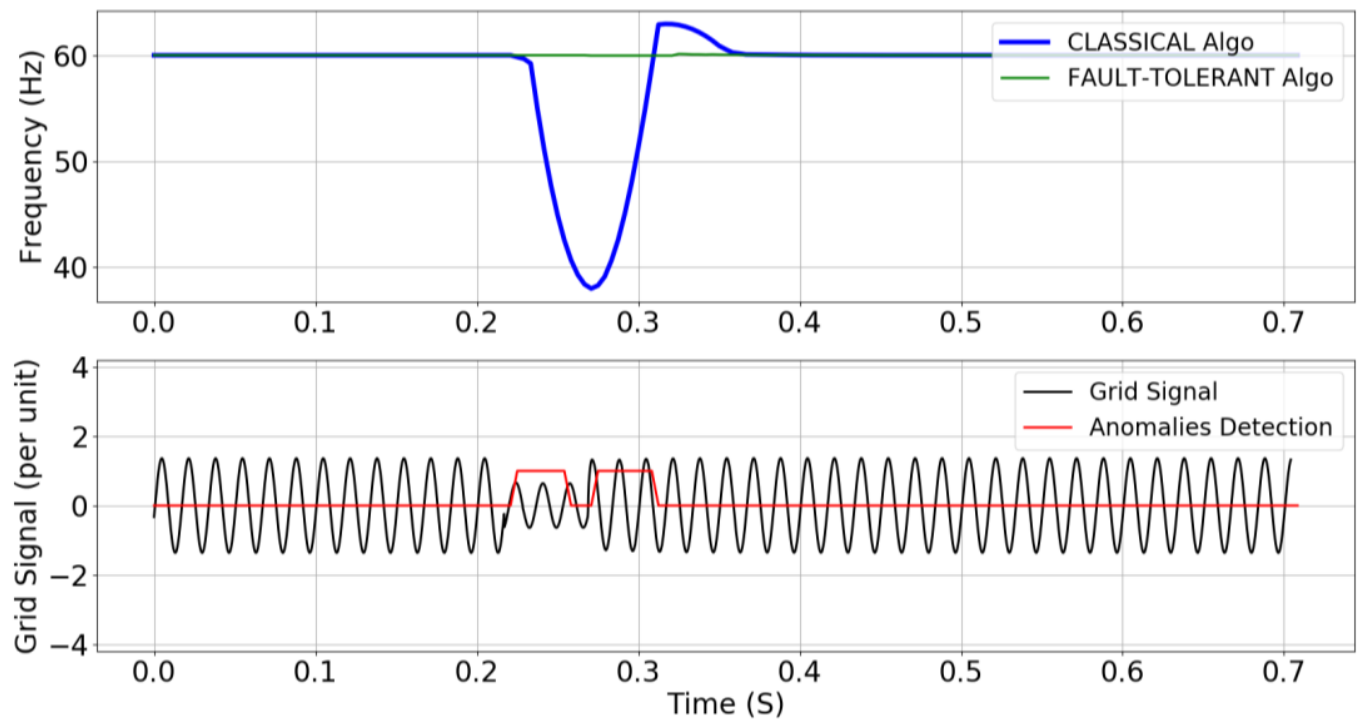

Figure 51 time domain result - case 37 


\subsubsection{Security test of fault-tolerant algorithm}

Now we have tested the dependability performance of the fault-tolerant algorithm under system transient faults. By performing dependability tests, we demonstrated that the fault-tolerant measurement algorithm can successfully detect the occurrence of the system transients and take actions to significantly mitigate measurement errors.

Besides the dependability performance, another important performance of the fault-tolerant algorithm is security performance. The power grid could experience some other type of distortions such as oscillation, ramping up and down, harmonics, etc. For these distortions, although the power grid signals are not ideal sinusoidal, the state-of-the-art algorithm can usually accurately perform the measurements, so the transient detector of the fault-tolerant algorithm should not trigger and recognize these distortions as "fault". Although trigger under these conditions will not significantly cause large measurement errors as the measurements are locked to latest valid measurement, it will fail to provide real-time valid measurements. Therefore, the fault-tolerant algorithm shall not "kick-in" when the grid experiences the distortions when the classical algorithm can perform well.

The IEEE Standard C37.118.1-2011 [22][18] and C37.118.1a-2014 [25].[25] provides a reference for such tests. It has various test signals that classical algorithm shall handle well. A detailed introduction of each test and the testing results are introduced below.

\section{Harmonic test}

The signal is represented as follows:

$$
s(t)=\cos (2 \pi f t)+0.1 \cos (2 \pi i f t)
$$

where $s(t)$ represents the test signal; $\cos (2 \pi f t)$ is the fundamental component of the test signal; $f$ is the fundamental frequency of the grid signal, $i$ is the order of the harmonic component, and $10 \%$ harmonic component, which is much higher than real-world grid environment, is assumed.

The testing results are shown in Figure 52 to Figure 63 with different combinations of $f$ and $i$. We can see the frequency measurements of the classical and fault-tolerant algorithm can match well each other, and it can be concluded that the fault-tolerant frequency algorithm performs stably and did not mistakenly recognize harmonic as transient faults.
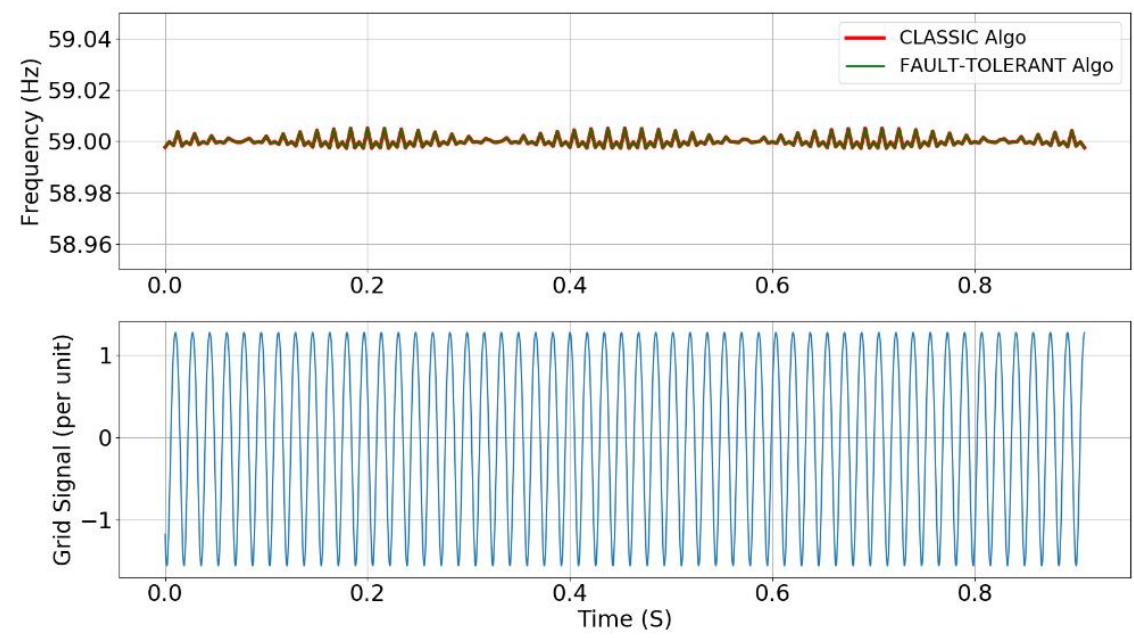

Figure 52 harmonic test $(f=59, i=2)$ 

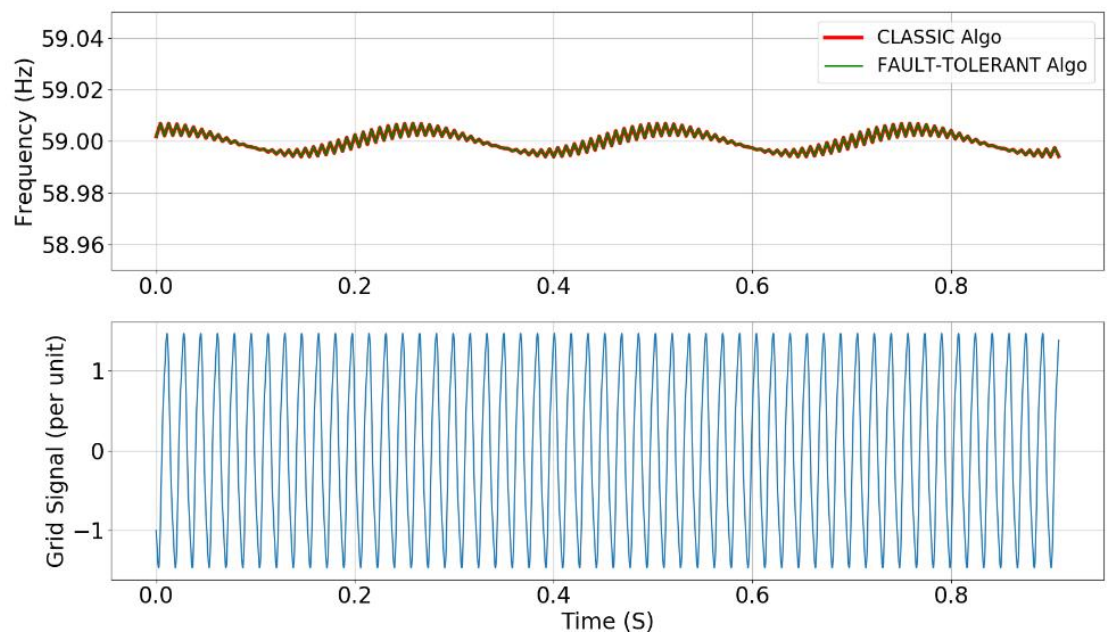

Figure 53 harmonic test $(f=59, i=3)$
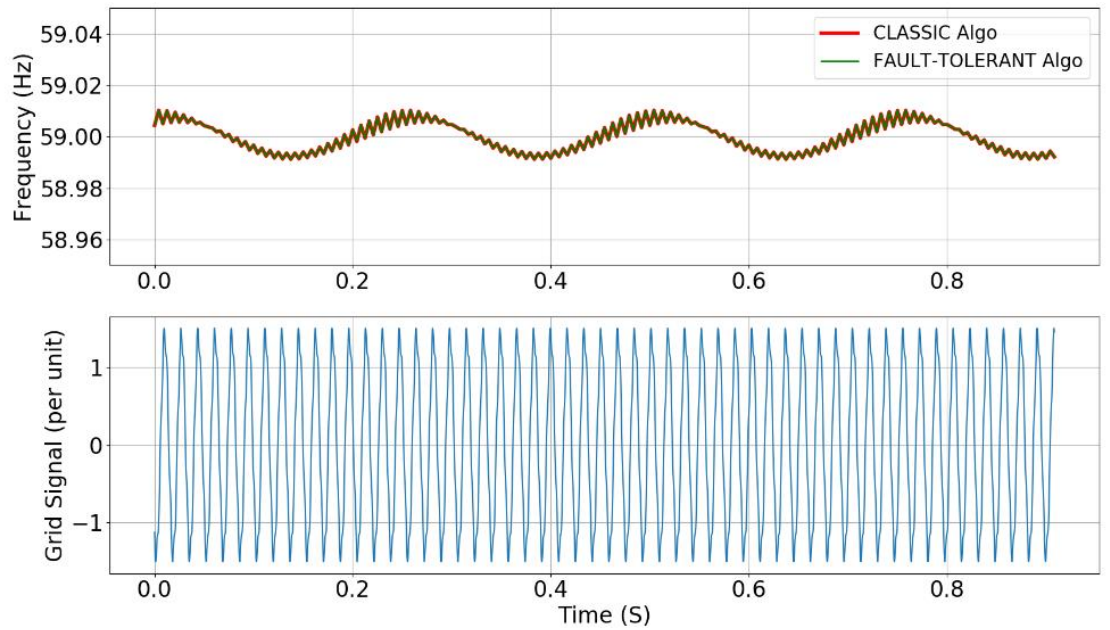

Figure 54 harmonic test $(f=59, i=5)$
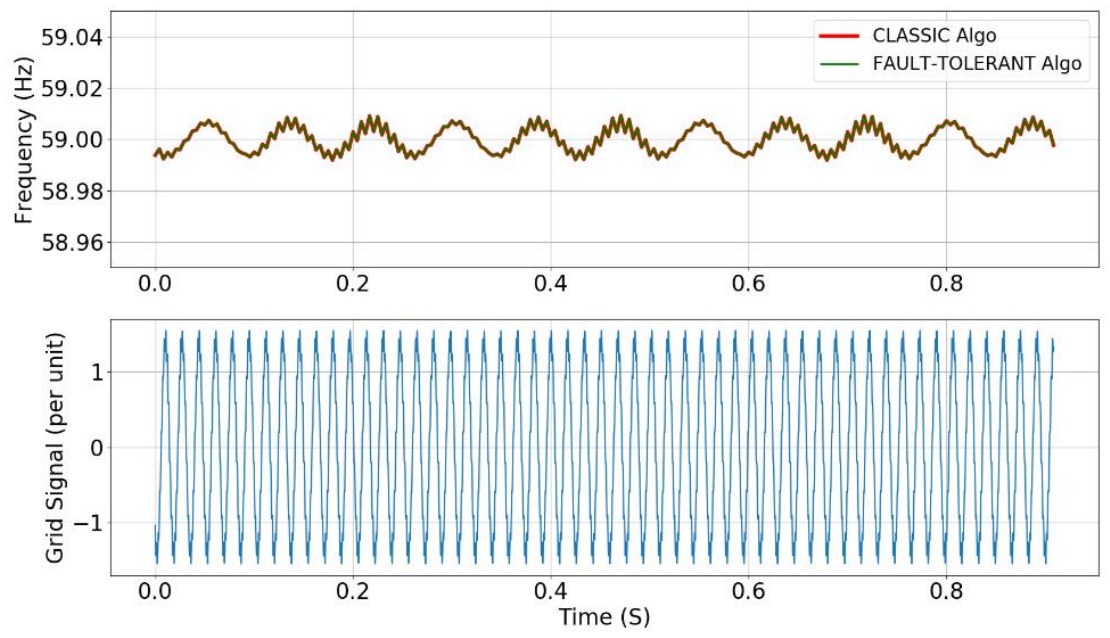

Figure 55 harmonic test $(f=59, i=7)$ 

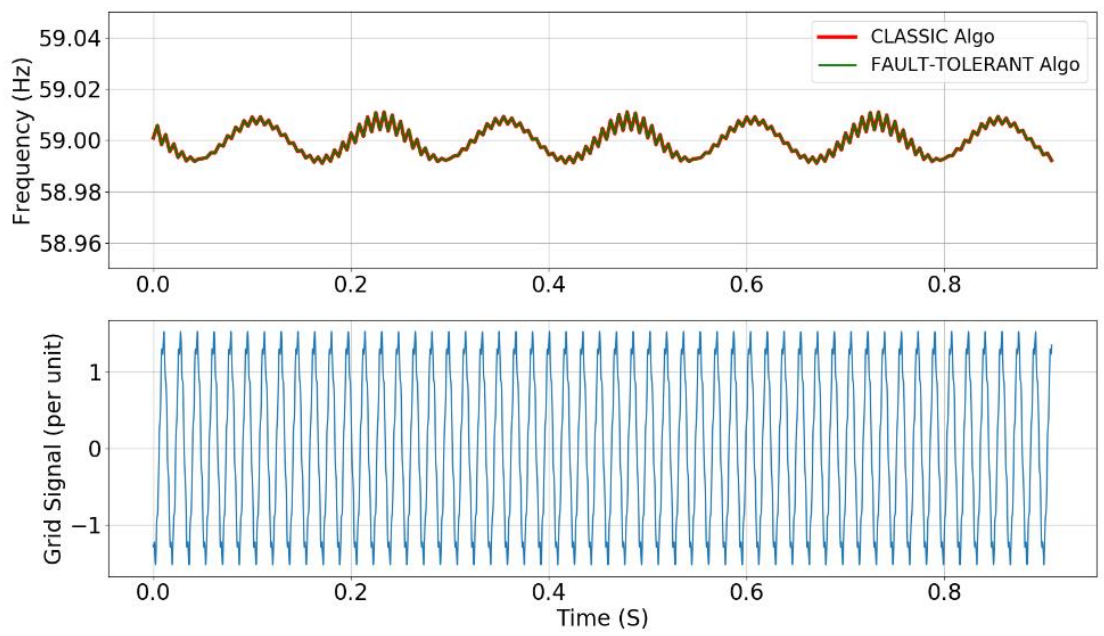

Figure 56 harmonic test $(f=59, i=11)$
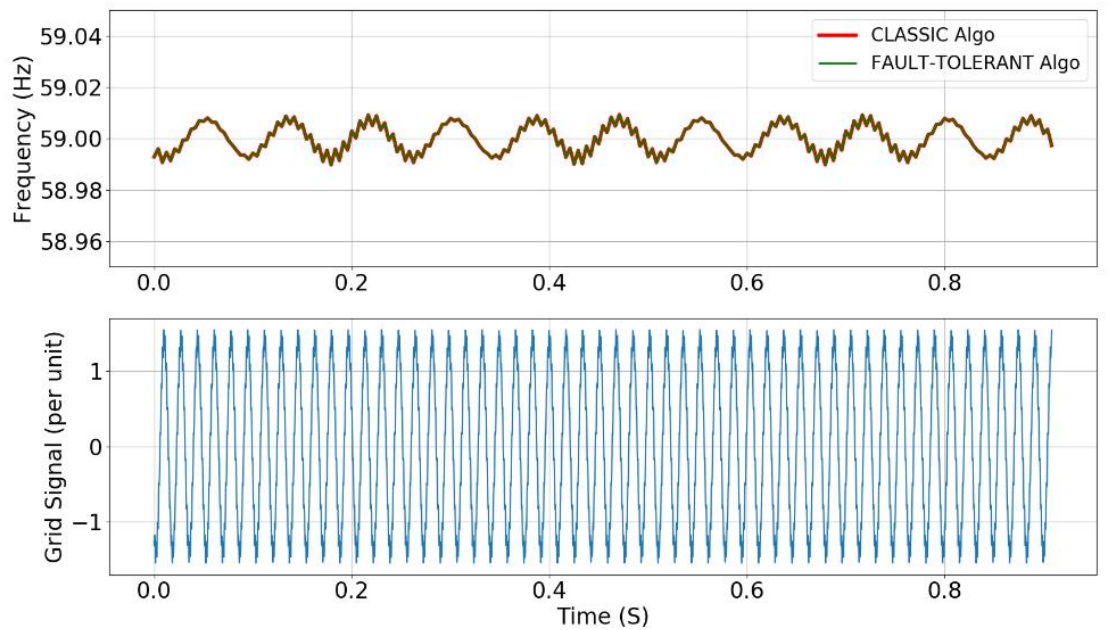

Figure 57 harmonic test $(f=59, i=13)$
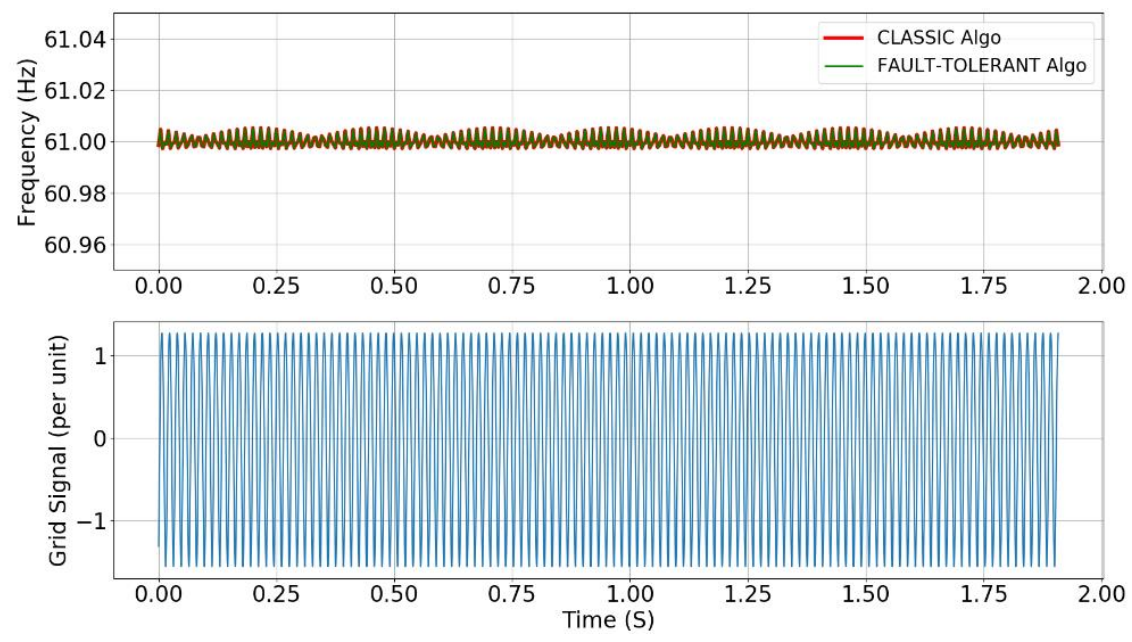

Figure 58 harmonic test $(f=61, i=2)$ 

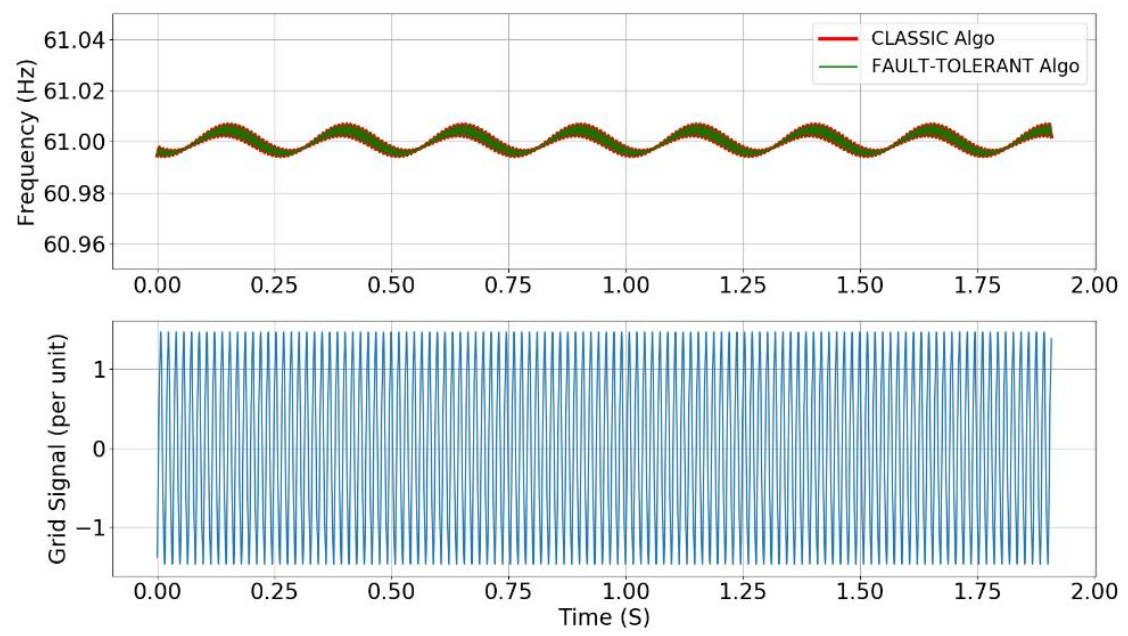

Figure 59 harmonic test $(f=61, i=3)$
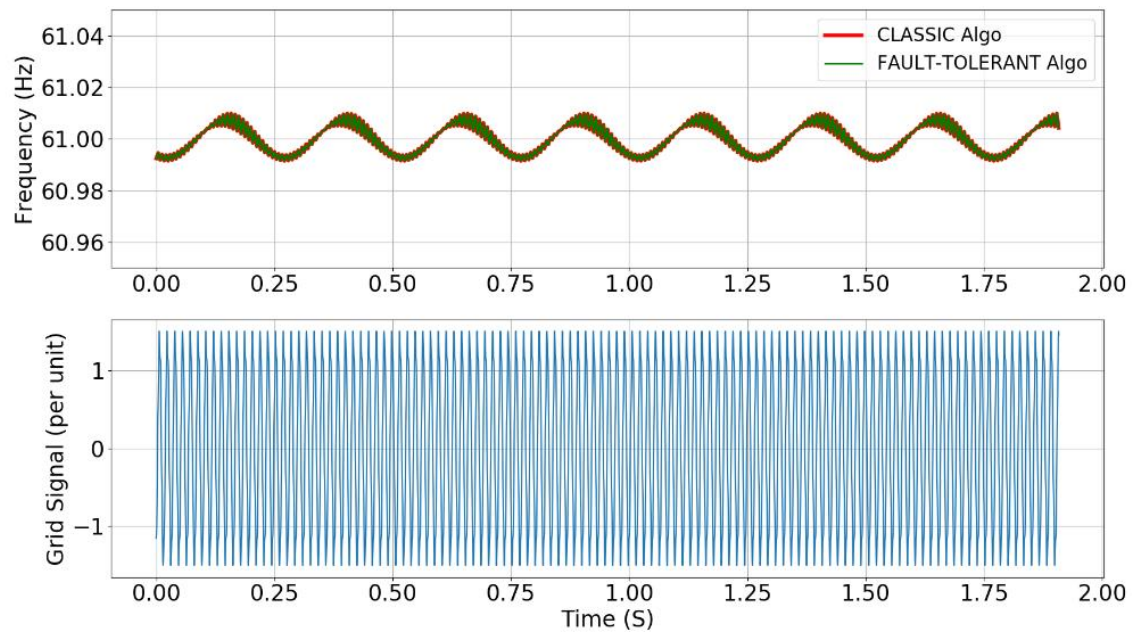

Figure 60 harmonic test $(f=61, i=5)$
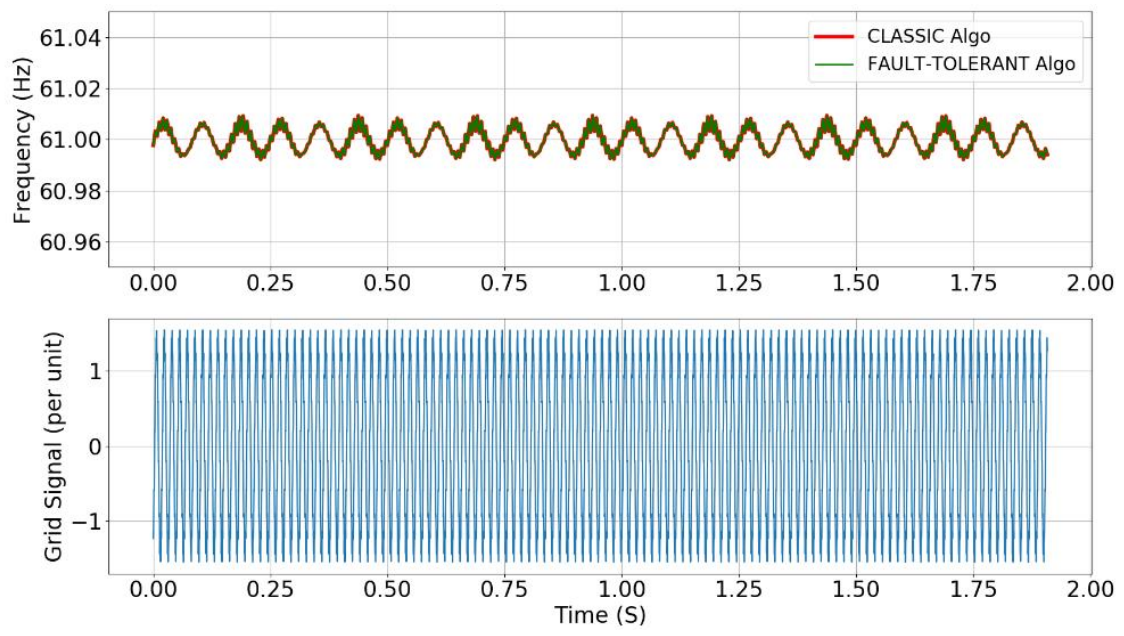

Figure 61 harmonic test $(f=61, i=7)$ 

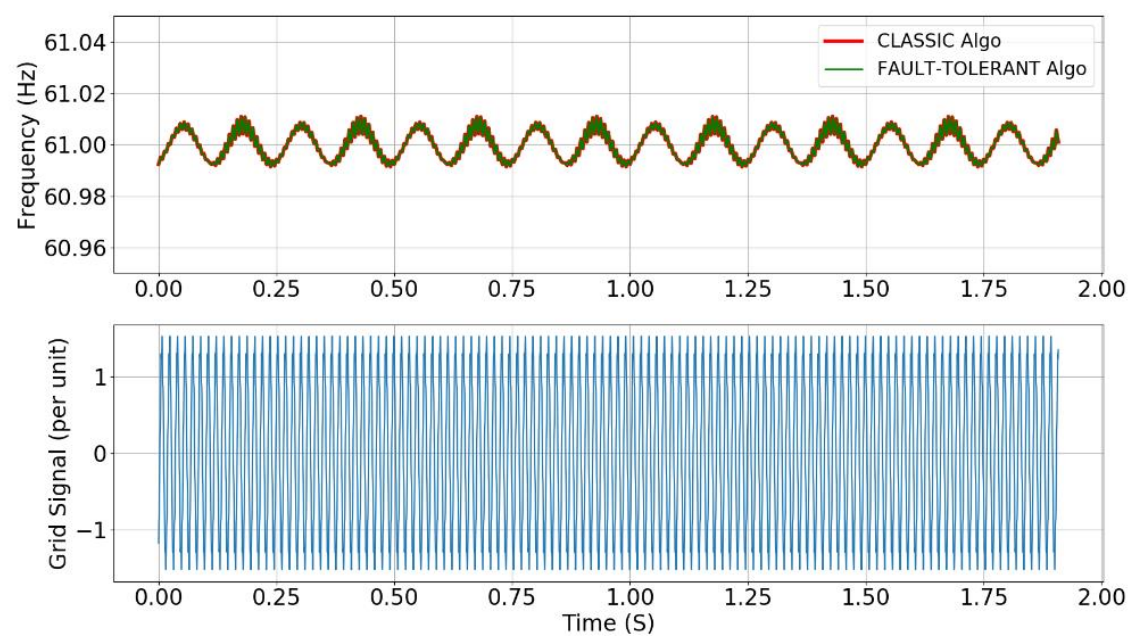

Figure 62 harmonic test $(f=61, i=11)$
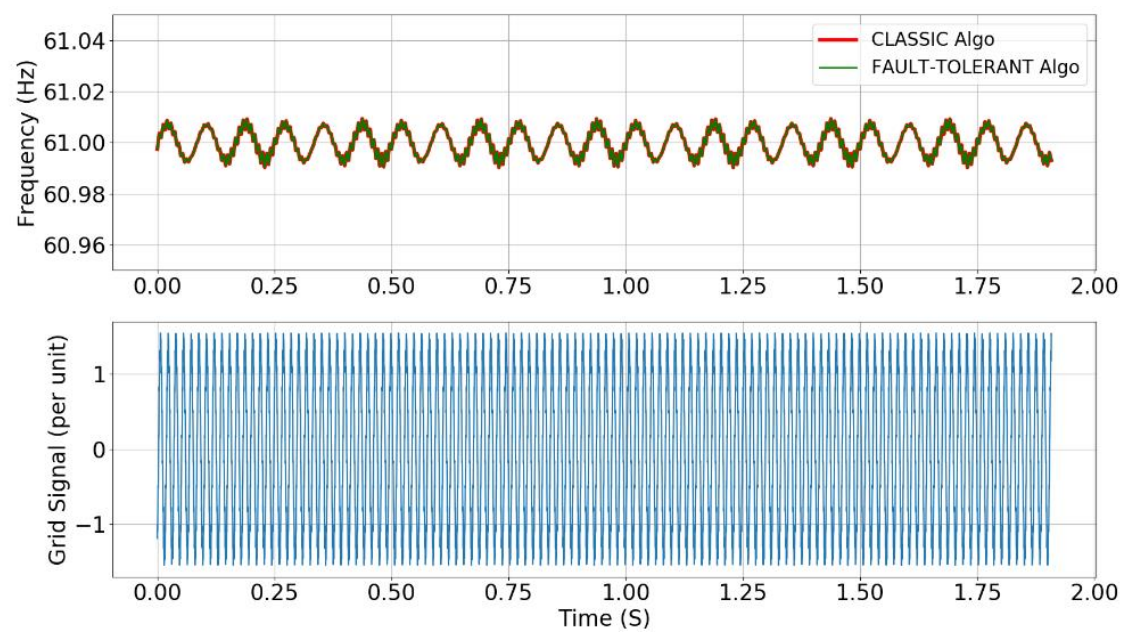

Figure 63 harmonic test $(f=61, i=13)$

\section{Phase angle modulation test}

The signal is represented as follows:

$$
s(t)=1 \cos \left(2 \pi f_{0} t+m_{a} \cos \left(2 \pi f_{a} t-\pi\right)\right)
$$

where $s(t)$ represents the test signal; $f_{0}$ is the nominal frequency of the grid signal (i.e., $60 \mathrm{~Hz}$ in United States), $m_{a}$ is the magnitude of the modulation signal, and $f_{a}$ is the modulation frequency of the modulation signal.

The testing results are shown in Figure 64 to Figure 70 with different combinations of $m_{a}$ and $f_{a}$. We can see the frequency measurements of the classical and fault-tolerant algorithm can match well each other, and it can be concluded that the fault-tolerant frequency algorithm performs stably and did not mistakenly recognize phase modulation signals as transient faults. 

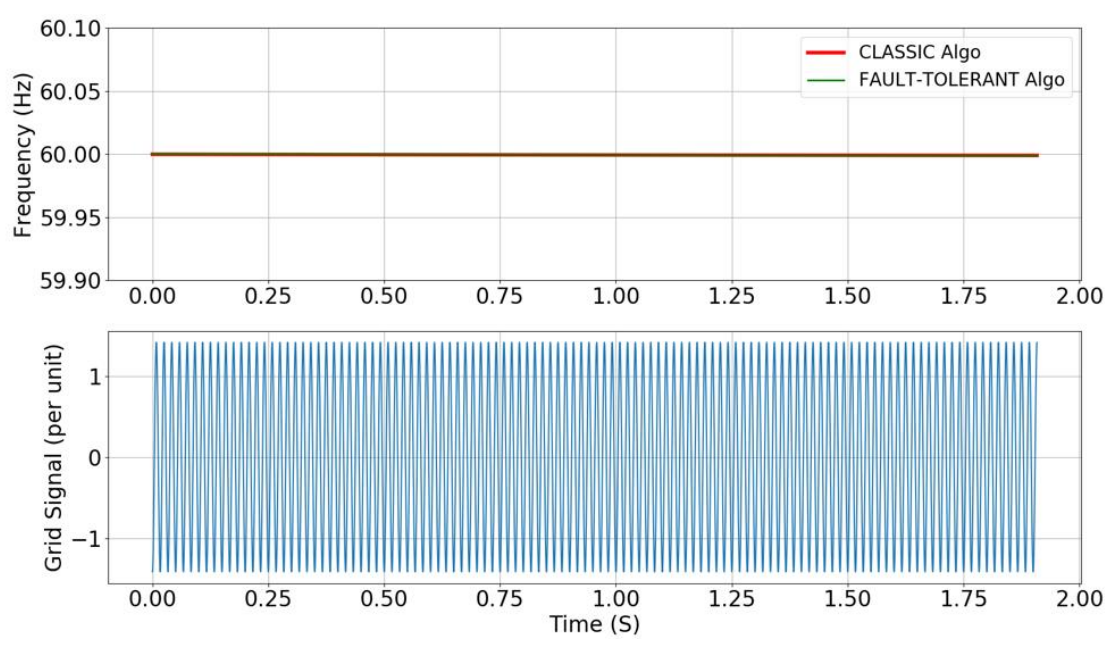

Figure 64 Phase modulation test $\left(m_{a}=0.01, f_{a}=0.1\right)$
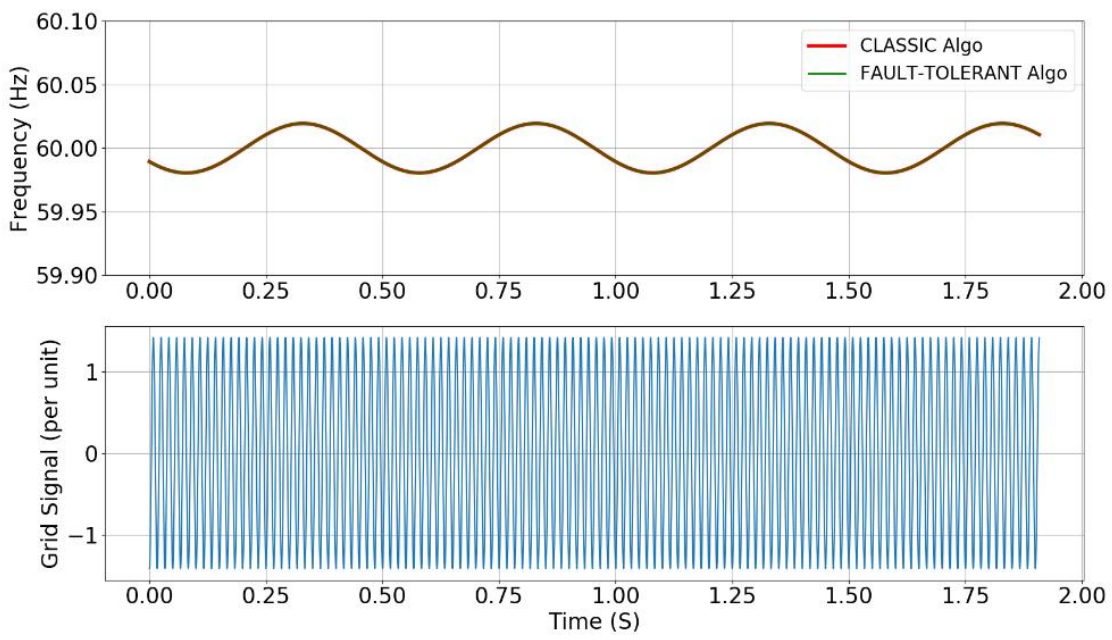

Figure 65 Phase modulation test $\left(m_{a}=0.01, f_{a}=2\right)$
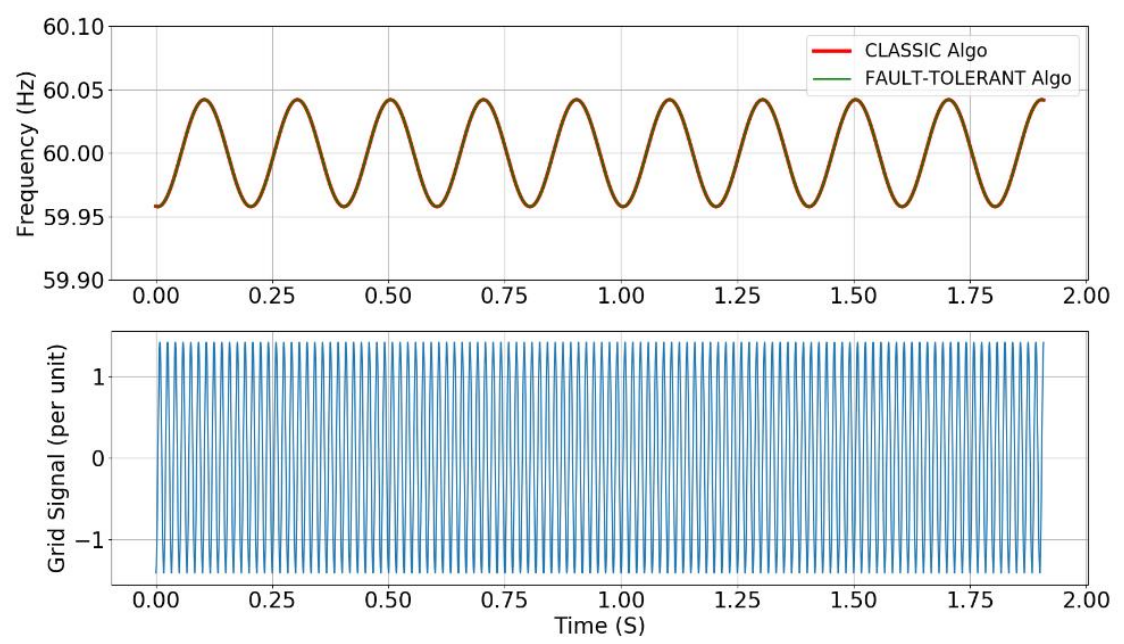

Figure 66 Phase modulation test $\left(m_{a}=0.01, f_{a}=5\right)$ 

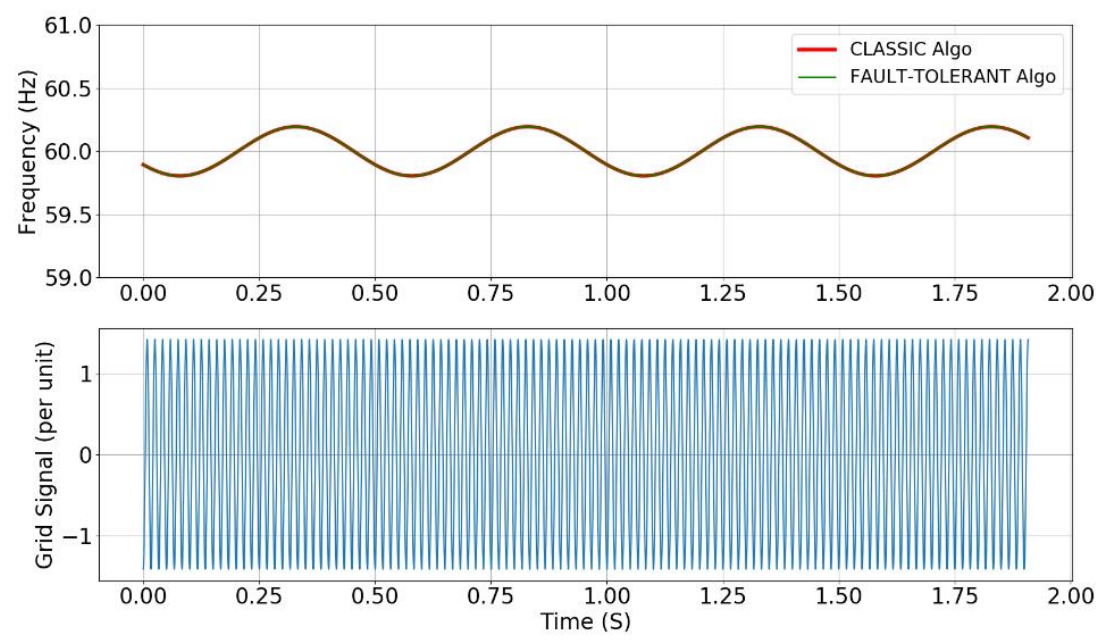

Figure 67 Phase modulation test $\left(m_{a}=0.1, f_{a}=2\right)$
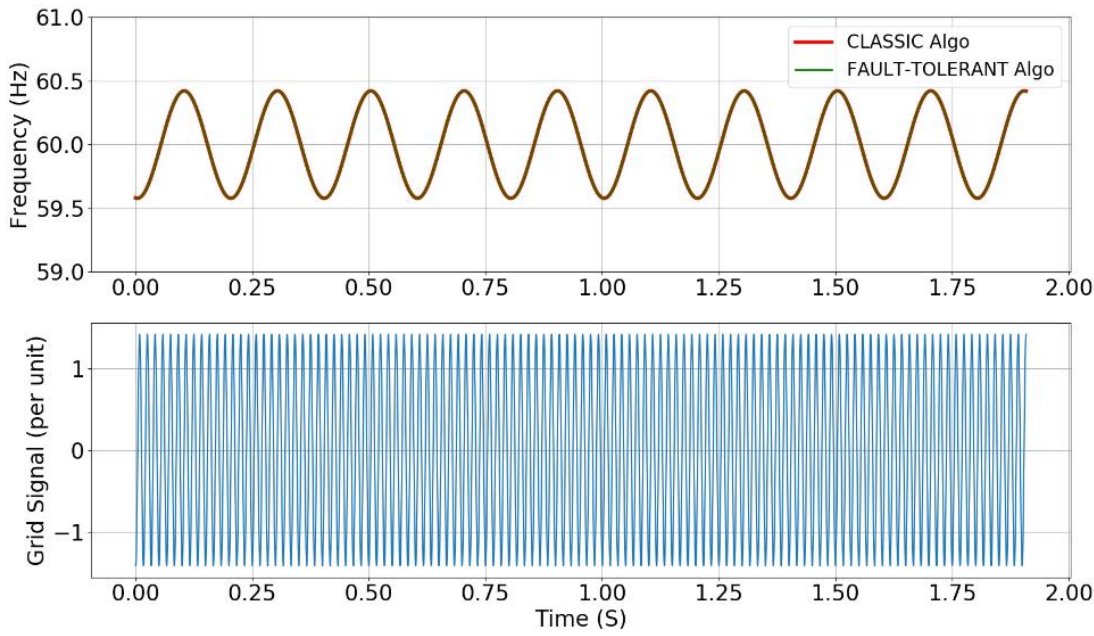

Figure 68 Phase modulation test $\left(m_{a}=0.1, f_{a}=5\right)$
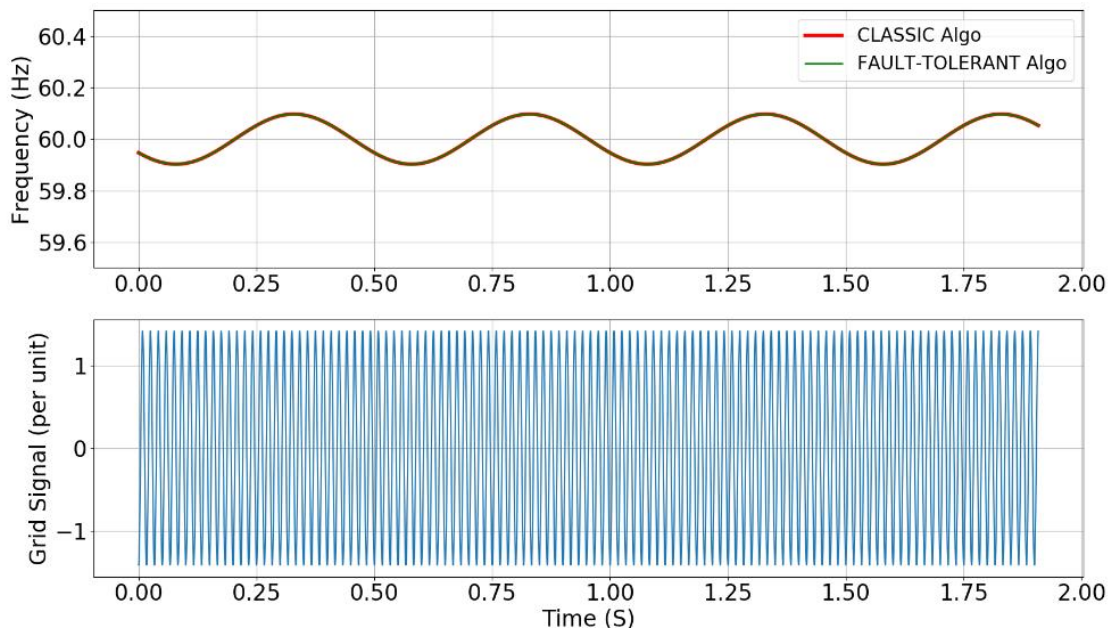

Figure 69 Phase modulation test $\left(m_{a}=0.1, f_{a}=5\right)$ 

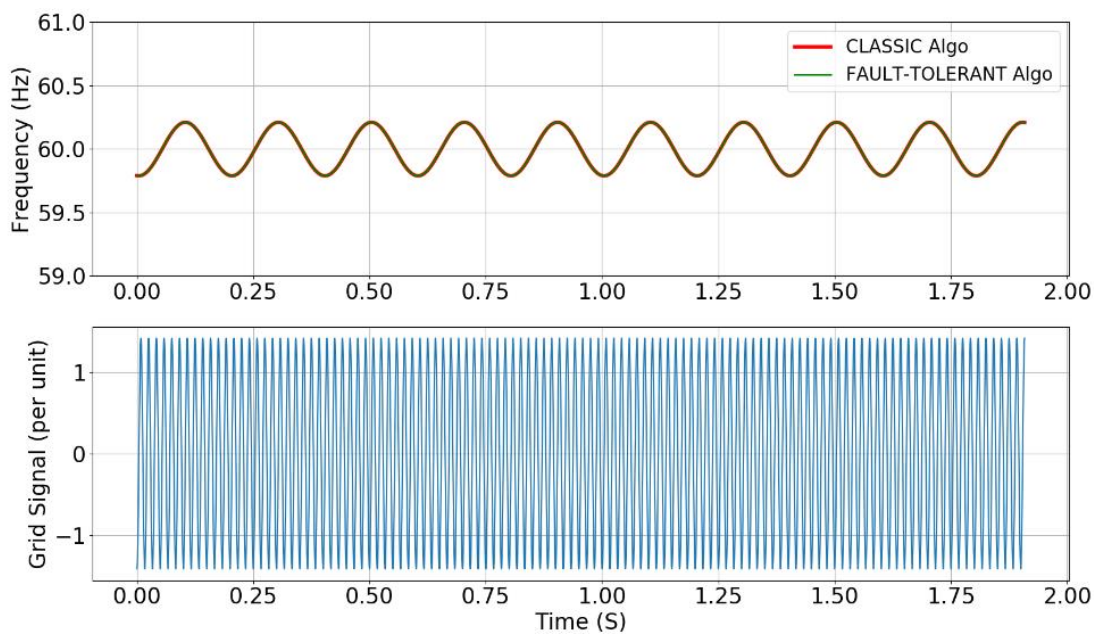

Figure 70 Phase modulation test $\left(m_{a}=0.05, f_{a}=5\right)$

\section{Magnitude modulation test}

The signal is represented as follows:

$$
s(t)=\left(1+m_{m} \cos \left(2 \pi f_{m} t\right)\right) * \cos \left(2 \pi f_{0} t\right)
$$

where $s(t)$ represents the test signal; $f_{0}$ is the nominal frequency of the grid signal (i.e. $60 \mathrm{~Hz}$ in United States), $m_{a}$ is the magnitude of the modulation signal, and $f_{m}$ is the modulation frequency of the modulation signal.

The testing results are shown in Figure 71 to Figure 79 with different combinations of $m_{m}$ and $f_{m}$. We can see the frequency measurements of the classical and fault-tolerant algorithm can match well each other, and it can be concluded that the fault-tolerant frequency algorithm performs stably and did not mistakenly recognize magnitude modulation signals as transient faults.
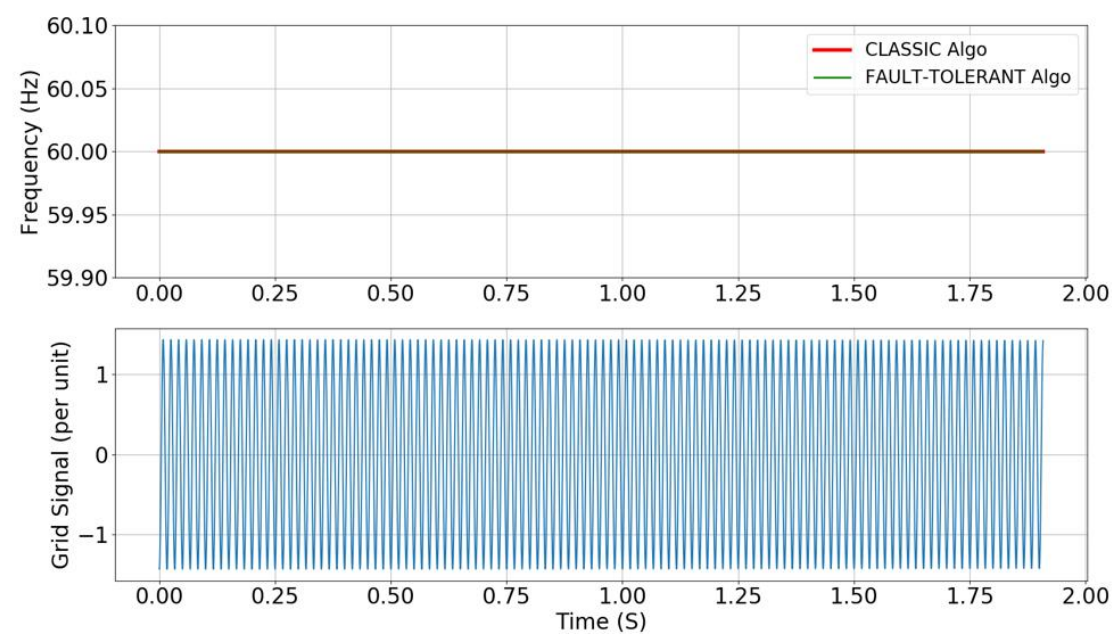

Figure 71 Magnitude modulation test $\left(m_{m}=0.01, f_{m}=0.1\right)$ 

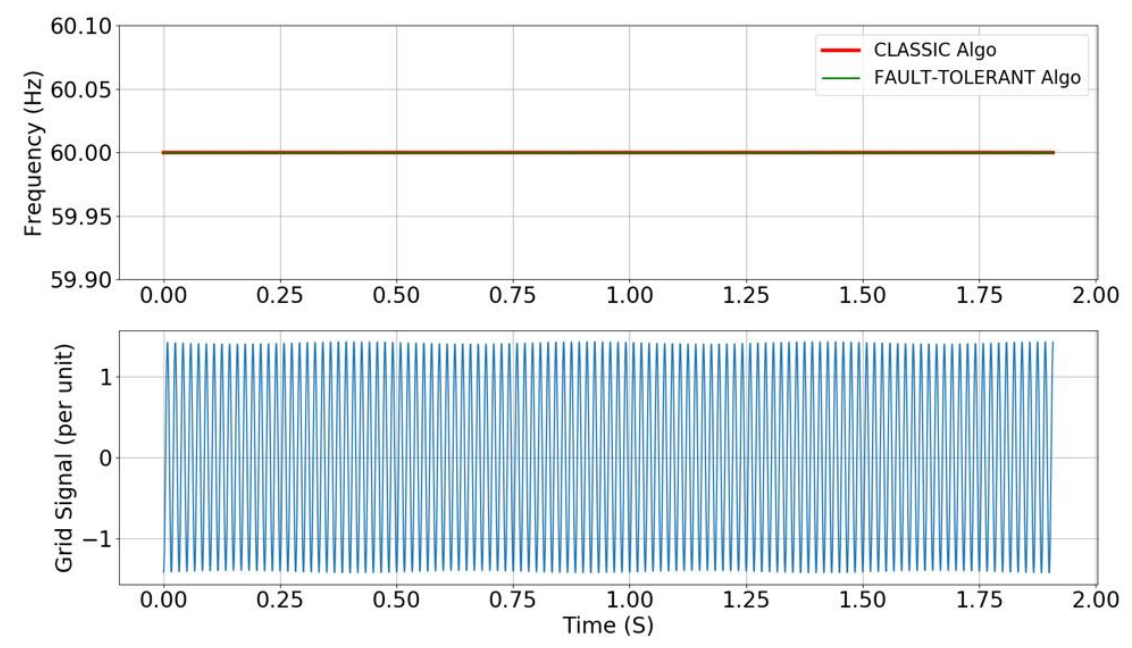

Figure 72 Magnitude modulation test $\left(m_{m}=0.01, f_{m}=2\right)$
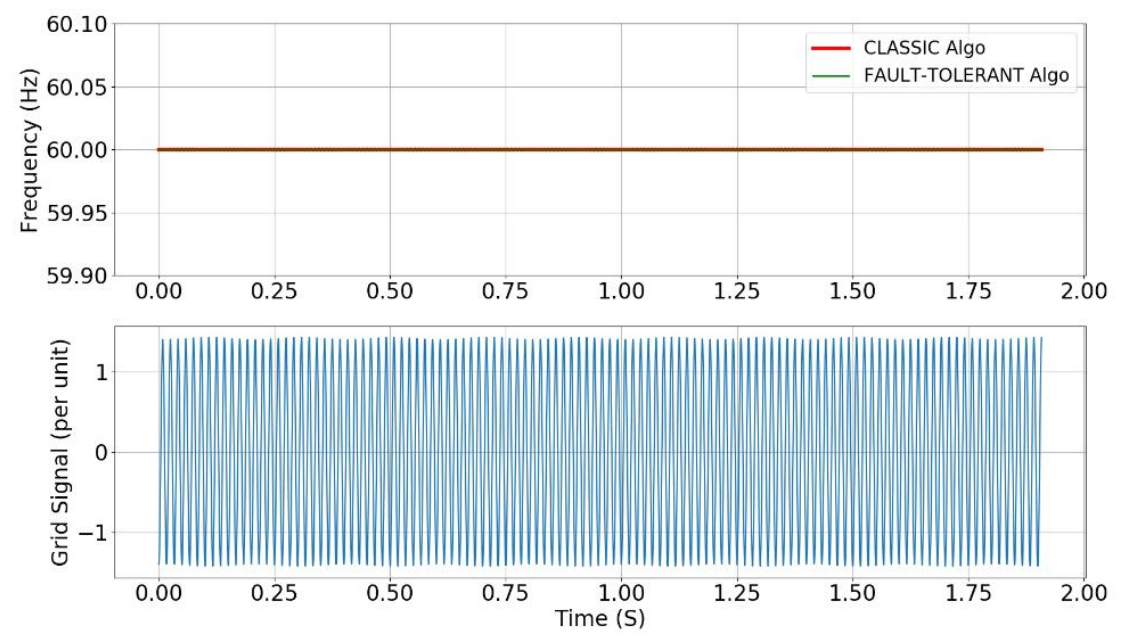

Figure 73 Magnitude modulation test $\left(m_{m}=0.01, f_{m}=5\right)$
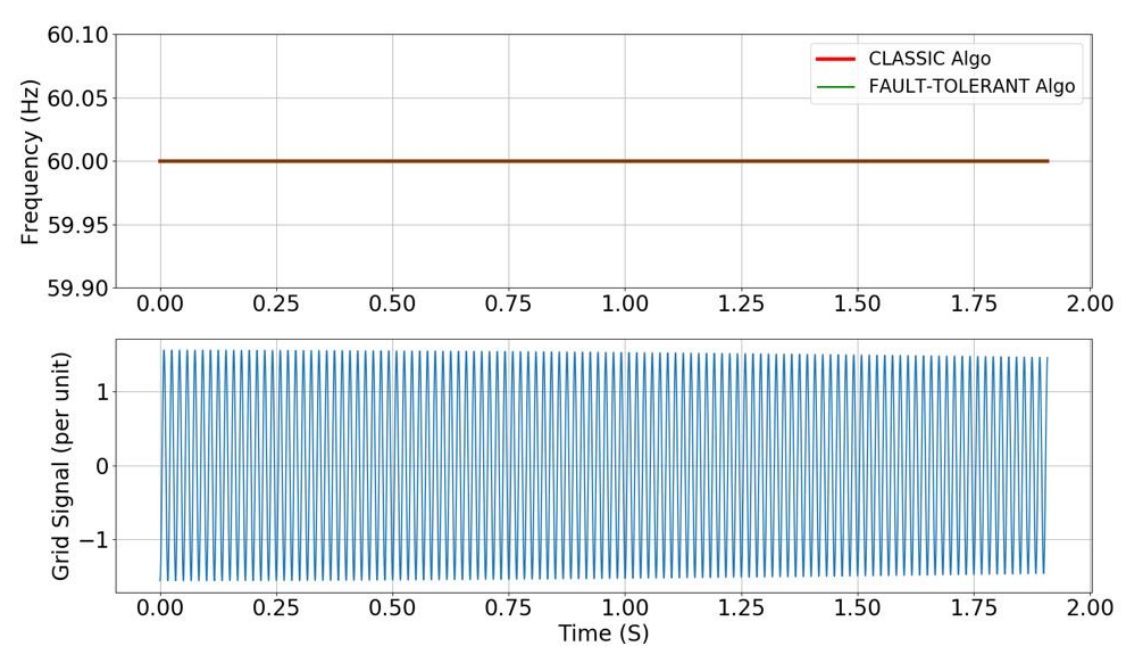

Figure 74 Magnitude modulation test $\left(m_{m}=0.1, f_{m}=0.1\right)$ 

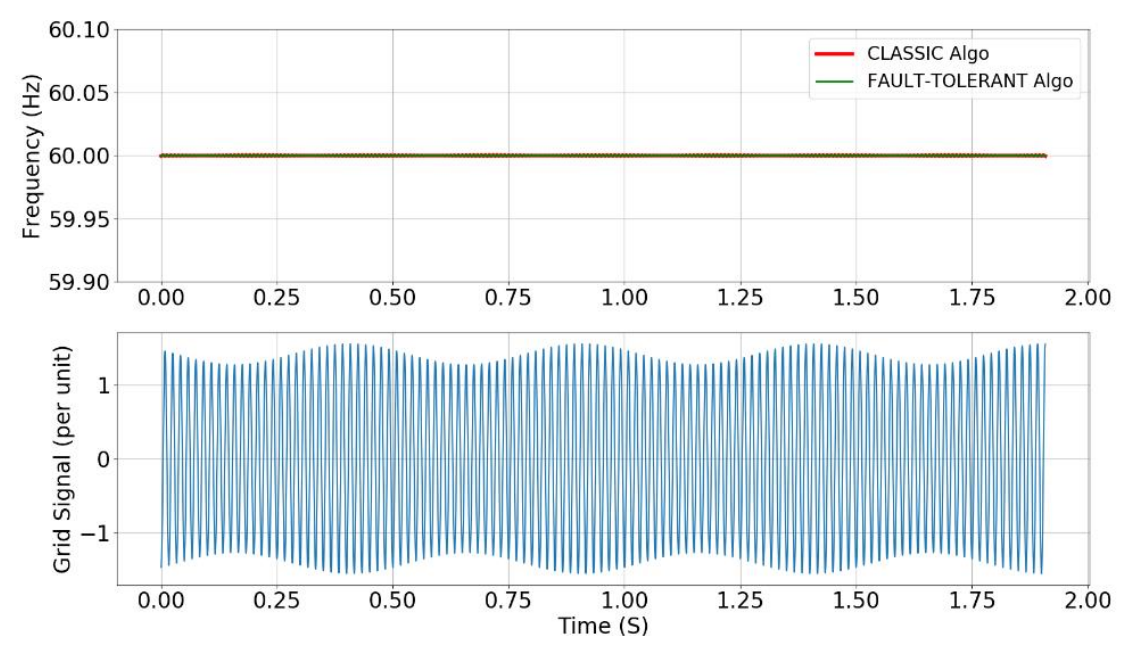

Figure 75 Magnitude modulation test $\left(m_{m}=0.1, f_{m}=2\right)$
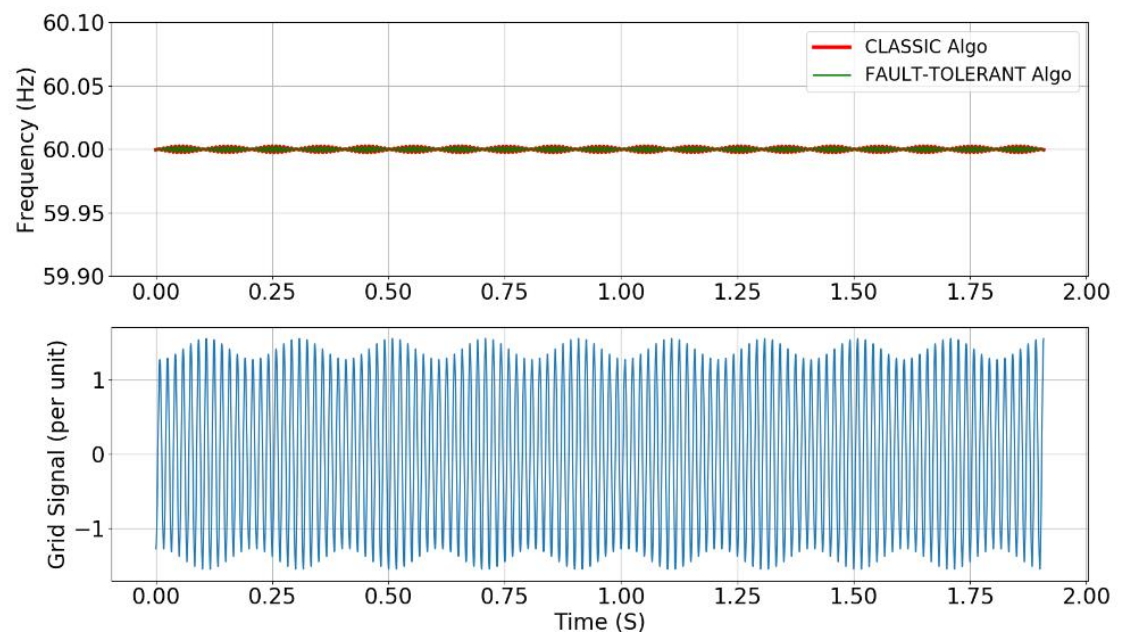

Figure 76 Magnitude modulation test $\left(m_{m}=0.1, f_{m}=5\right)$
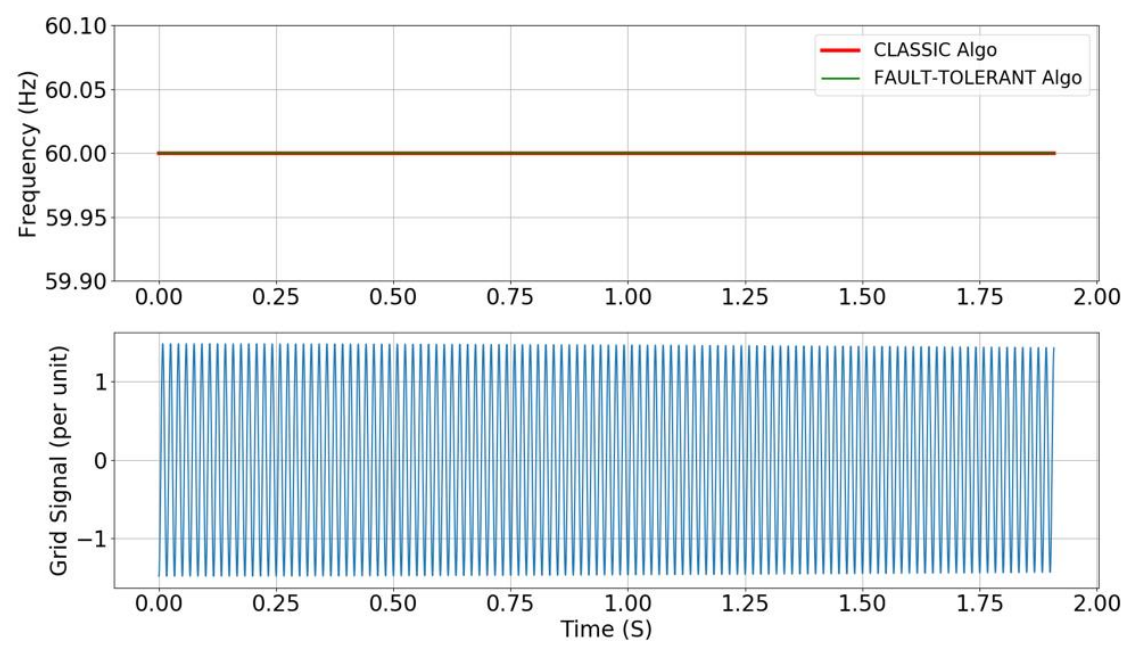

Figure 77 Magnitude modulation test $\left(m_{m}=0.05, f_{m}=0.1\right)$ 

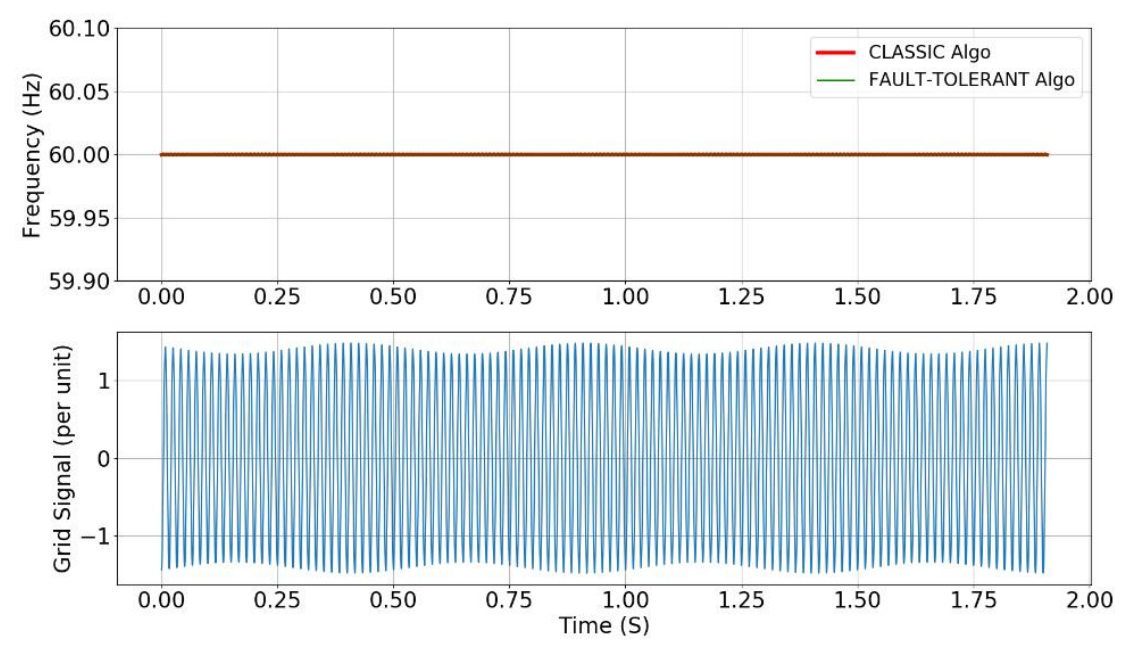

Figure 78 Magnitude modulation test $\left(m_{m}=0.05, f_{m}=2\right)$
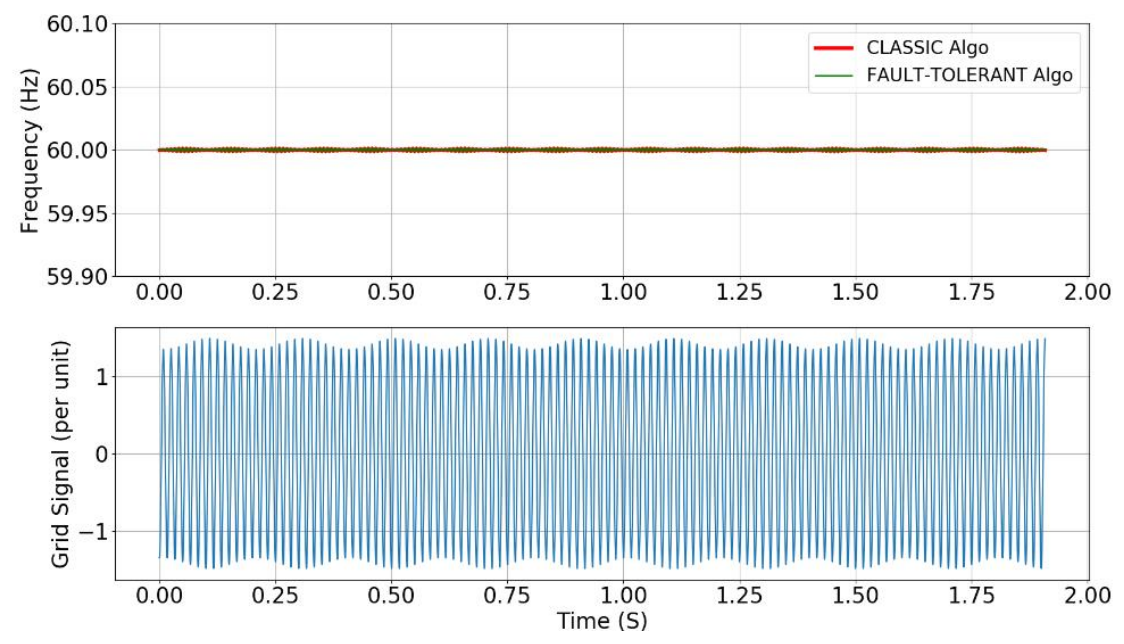

Figure 79 Magnitude modulation test $\left(m_{m}=0.05, f_{m}=5\right)$

\section{Magnitude ramping test}

Note: this test is not specified in IEEE Std., however we believe it is a practical scenario that can happen in real-world, so we did the test.

$$
s(t)=\left(M_{s}+r * t\right) \cos (2 \pi f t)
$$

where $M_{s}$ is the initial magnitude at $t=0 ; r$ is the rate of magnitude ramping.

The testing results are shown in Figure 80 to Figure 83 with different combinations of $M_{s}$ and $r$. We can see the frequency measurements of the classical and fault-tolerant algorithm can match well each other, and it can be concluded that the fault-tolerant frequency algorithm performs stably and did not mistakenly recognize magnitude ramping signals as transient faults. 

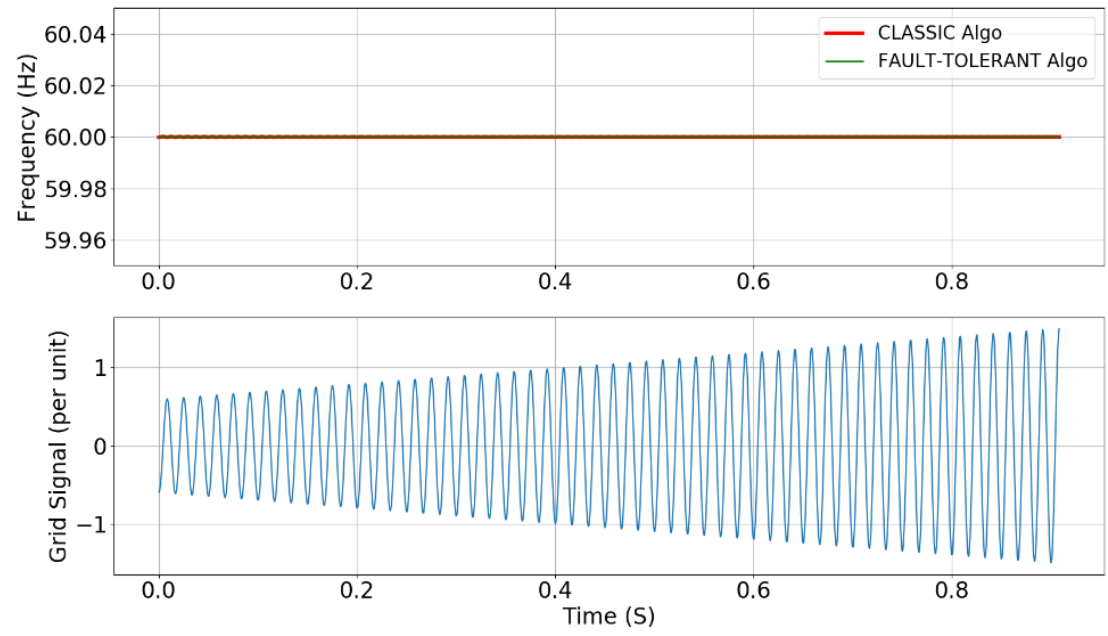

Figure 80 Magnitude ramping test $\left(M_{s}=0.5, r=1, f=60\right)$
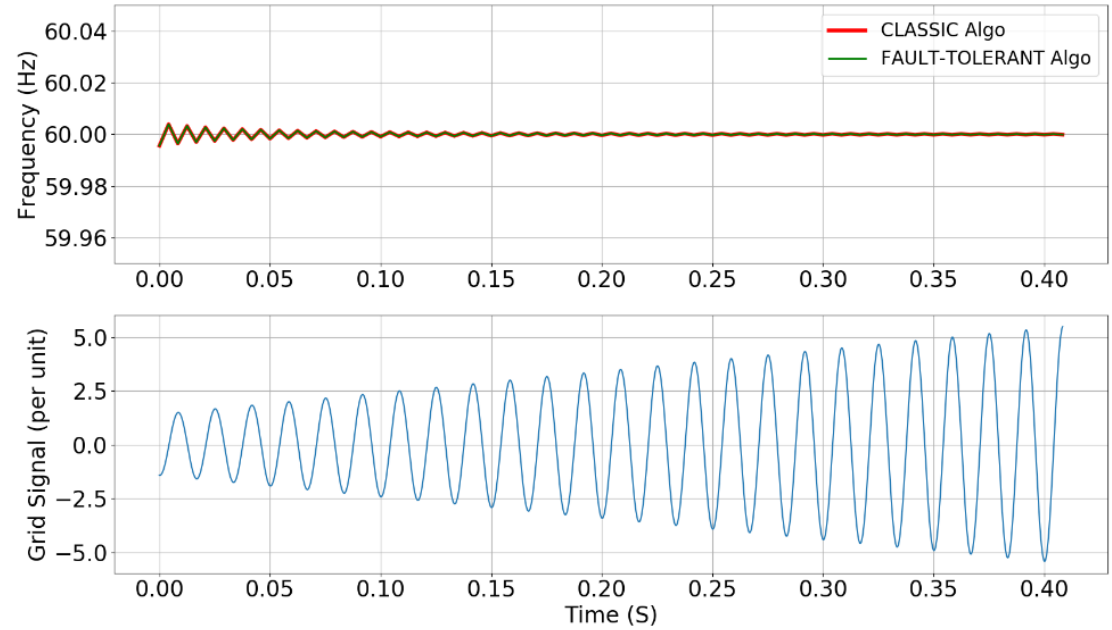

Figure 81 Magnitude ramping test $\left(M_{s}=0.5, r=5, f=60\right)$
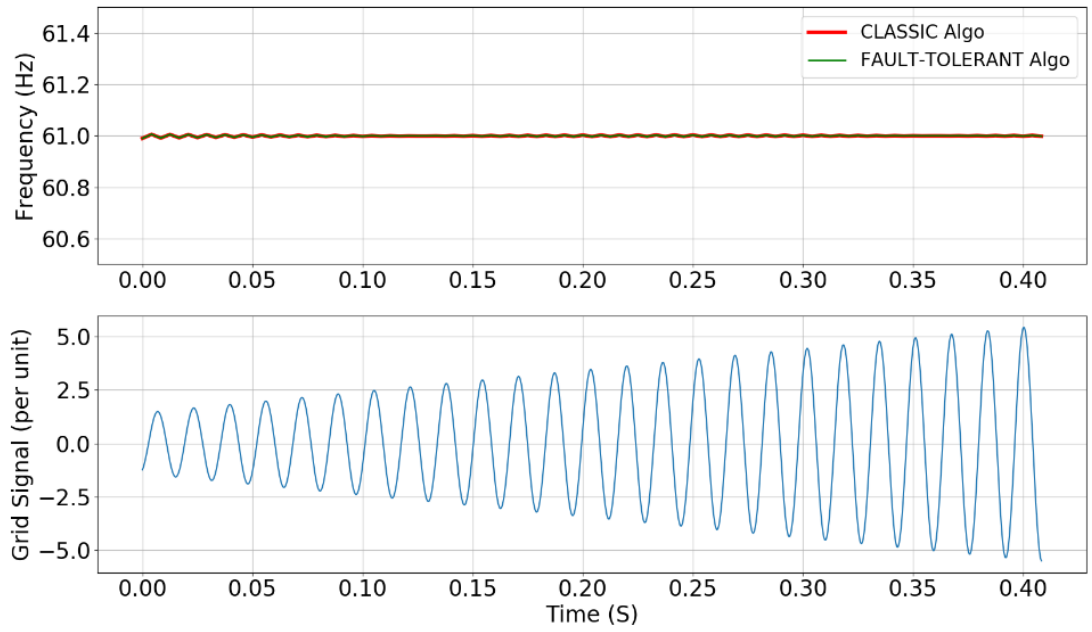

Figure 82 Magnitude ramping test $\left(M_{s}=0.5, r=5, f=61\right)$ 

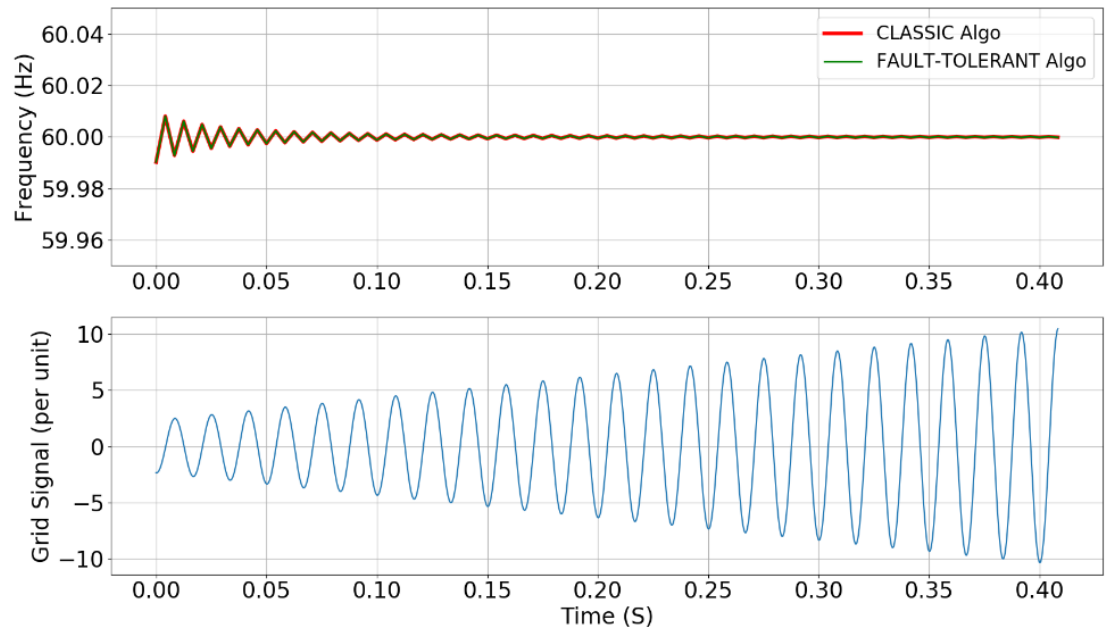

Figure 83 Magnitude ramping test $\left(M_{s}=0.5, r=10, f=60\right)$

\section{Frequency ramping test}

$$
s(t)=1 \cos (2 \pi(59+0.5 t) t)
$$

In this test, the frequency first ramps up from $59 \mathrm{~Hz}$ to $61 \mathrm{~Hz}$ in 2 seconds, and stays at 61 for a second, then ramps down from $61 \mathrm{~Hz}$ to $59 \mathrm{~Hz}$ in 2 seconds. We can see the frequency measurements of the classical and fault-tolerant algorithm can match well each other, and it can be concluded that the faulttolerant frequency algorithm performs stably and did not mistakenly recognize frequency ramping signals as transient faults.
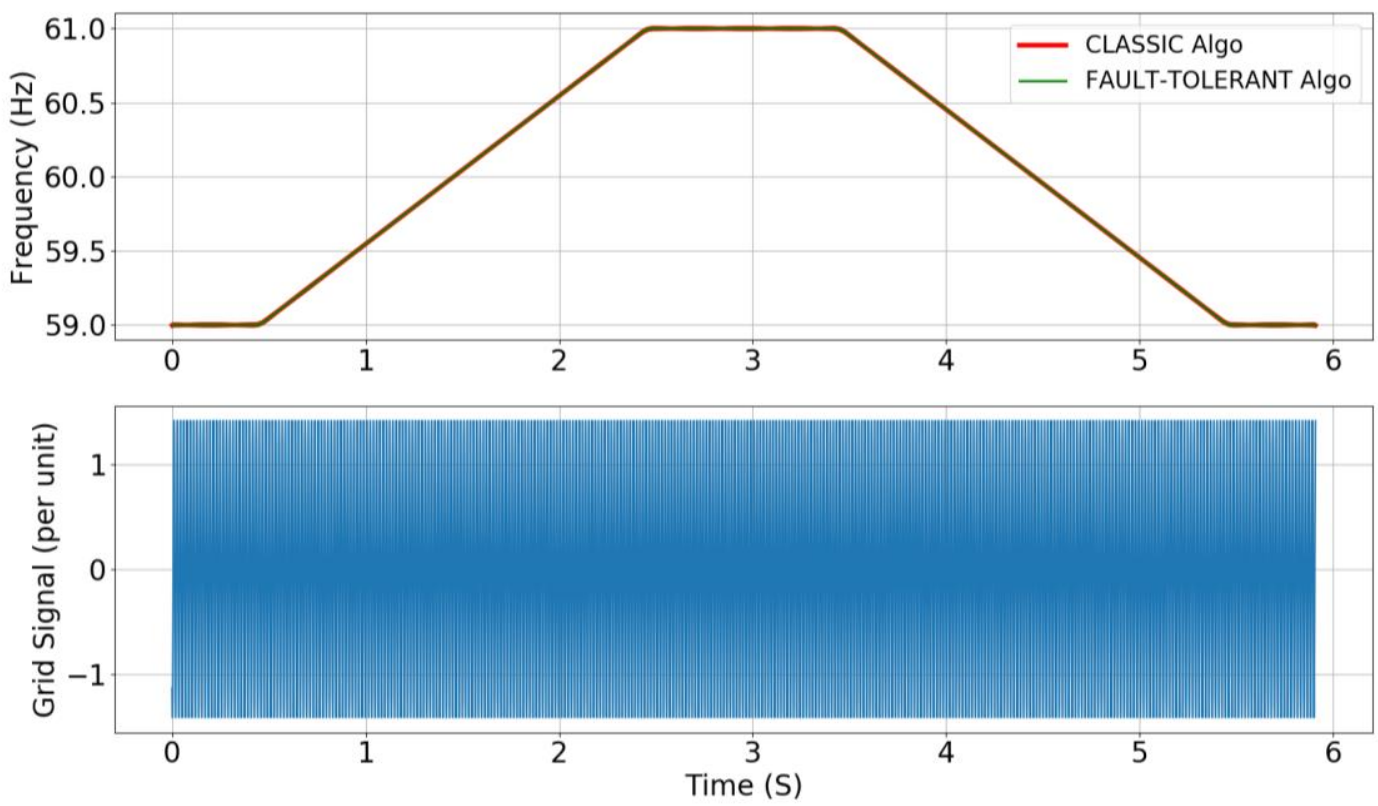

Figure 84 Frequency ramping test 


\subsection{COMPUTATION COMPLEXITY ANALYSIS AND COMPUTATION COST TEST}

To analyze the applicability of the new algorithm on industry products, the computational complexity of the algorithm was analyzed in detail in terms of number of numerical operations, and the computation capacity of industry widely used microprocessor Texas Instruments TMS320VC33 is used for analysis, which has 150 million Floating-Point Operations Per Second (MFLOPS). The computation time of the algorithm was tested by considering different combination of sampling rate, window size.

The proposed fault-tolerant estimator can be divided into two parts. The first part is the transient detector, and the second part is the frequency estimator. They will be analyzed separately.

\subsubsection{Transient Detector}

\subsubsection{Computational Complexity of The Transient Detector}

A flowchart of the transient detector is shown in Figure 85.

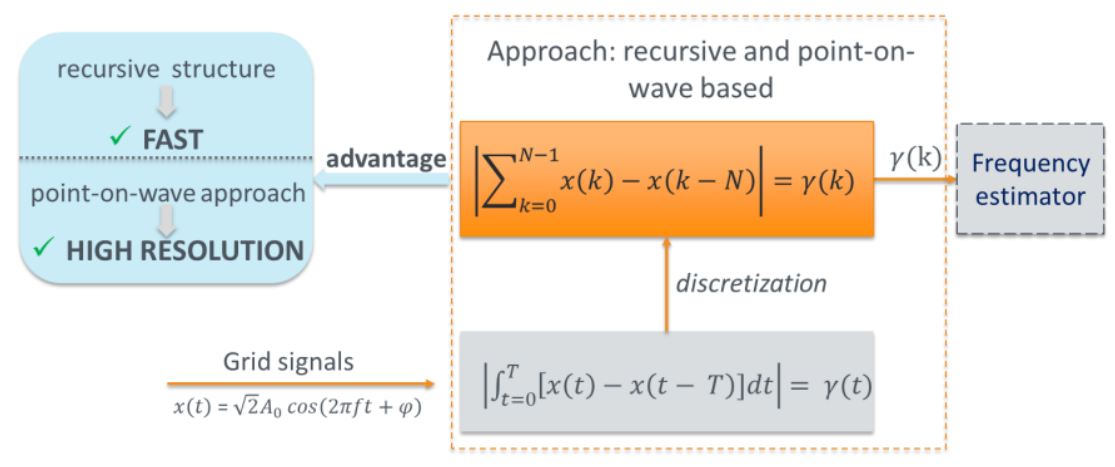

Figure 85 Flowchart of the transient detector

The transient detector runs in real-time at a rate of sampling rate, which can be expressed by

$$
\gamma(i)=\left|\sum_{k=0}^{N-1} x(i+k)-x(i+k-N)\right|
$$

where $x$ is the sampled value of the power grid electric waveforms, $N$ is the number of sampled values in one fundamental frequency cycle (i.e., $1 / 60$ second for a $60 \mathrm{~Hz}$ power system). The computation time of (10) is proportional to $N$, which will increase with the sampling rate. However, assuming

$$
s(i)=\sum_{k=0}^{N-1} x(i+k)-x(i+k-N)
$$

(11) can be rewritten in the following form

$$
s(i)=\left\{\begin{array}{c}
s(i-1)+\{x(i+N)-x(i)\}-\{x(i)-x(i-N)\}, \quad i>0 \\
\sum_{k=0}^{N-1} x(k)-x(k-N), \quad i=0
\end{array}\right.
$$

According to (12), the number of numerical operations to calculate $s(0)$ is proportional to $N$. Once we have $s(0), s(i)$ can be calculated recursively. To be specific, to calculate $s(i)$ from $s(i-1)$, we only one 
"addition", and three "subtraction". And to calculate $\gamma(i)$ from $s(i)$, one 'abs' operation is needed. Table VII summarizes the count of numerical operations of the transient detector.

Table VII Count of numerical operations of the transient detector

\begin{tabular}{|c|c|c|c|}
\hline & count of "+" operation & count of “-” operation & $\begin{array}{c}\text { count of "abs" } \\
\text { operation }\end{array}$ \\
\hline Transient detector & 1 & 3 & 1 \\
\hline
\end{tabular}

According to the analysis and Table VII, we can conclude that the computational complexity of the transient detector in Big $O$ notation is $O(1)$, which is constant time and independent of sampling rate or computation window. As only a few numbers of numerical operations are needed, the computation will be very fast.

The modern digital signal processors can perform one or more numerical operations (e.g., ' + , $’$, and 'abs') per microprocessor cycle, thus, a new transient detector result can be computed within a few microprocessor cycles.

\subsubsection{Computation Time Analysis on Digital Signal Processor}

Digital Signal Processor (DSP) is usually used for measurement computation. Table VIII shows the calculation of the computation utilization rate of the transient detector on a typical DSP. Assuming the sampling rate is $6000 \mathrm{~Hz}$, the total number of numerical operations of the transient detector is about 30,000 , which is only $0.02 \%$ of the computing capability of the DSP.

Table VIII Computation utilization rate of the transient detector

\begin{tabular}{|l|l|l|l|l|l|}
\hline & $\begin{array}{l}\text { \# of numerical } \\
\text { operations per } \\
\text { measurement }\end{array}$ & $\begin{array}{l}\text { \# of measurements } \\
\text { per second }\end{array}$ & $\begin{array}{l}\text { Total \# of } \\
\text { numerical } \\
\text { operations } \\
\text { per second }\end{array}$ & $\begin{array}{l}\text { MFLOPS of the } \\
\text { DSP }\end{array}$ & $\begin{array}{l}\text { Computation } \\
\text { utilization rate }\end{array}$ \\
\hline $\begin{array}{l}\text { Transient } \\
\text { Detection }\end{array}$ & 5 & 6000 & 30,000 & $150,000,000$ & $0.02 \%$ \\
\hline
\end{tabular}

\subsubsection{Computation Time Test of The Transient Detector}

The computation time of the transient detector has also been evaluated on a $3.6 \mathrm{GHz} 4$-core microprocessor, and the testing results are shown in Table IX.

Different combinations of sampling rate and time of test signal are tested. According to Table IX, the total computation time increases with the sampling rate and time of test signal, which is expected. The key observation is the computation time per calculation in last column, which is almost constant regardless of sampling rate or time of test signal. It varies in a narrow range from 70 to 90 microsecond. At a sampling rate of $5760 \mathrm{~Hz}$, the computation time is about 0.9 microsecond. The low computation cost is very critical for real-time transient event detection. 
Table IX Computation time test of the transient detector

\begin{tabular}{|l|l|l|l|l|}
\hline \multirow{2}{*}{$\begin{array}{l}\text { Sampling } \\
\text { Rate }\end{array}$} & $\begin{array}{l}\text { Time of test } \\
\text { signal (Second) }\end{array}$ & $\begin{array}{l}\text { Number of } \\
\text { sampled values }\end{array}$ & Total (Second) & $\begin{array}{l}\text { Per calculation } \\
\text { (Microsecond) }\end{array}$ \\
\cline { 3 - 5 } & 1 & 1,440 & 0.001 & 0.73 \\
\hline \multirow{2}{*}{$1440 \mathrm{~Hz}$} & 10 & 14,400 & 0.012 & 0.84 \\
\cline { 2 - 5 } & 100 & 144,000 & 0.123 & 0.86 \\
\hline \multirow{2}{*}{$2880 \mathrm{~Hz}$} & 1 & 2,880 & 0.002 & 0.72 \\
\hline & 10 & 28,800 & 0.023 & 0.80 \\
\hline \multirow{2}{*}{$5760 \mathrm{~Hz}$} & 100 & 288,000 & 0.244 & 0.85 \\
\hline & 1 & 5,760 & 0.005 & 0.90 \\
\hline & 100 & 57,600 & 0.046 & 0.80 \\
\hline
\end{tabular}

\subsubsection{Frequency Estimator}

\subsubsection{Computational Complexity of The Frequency Estimator}

The frequency estimator is more complex than the transient detector. For a frequency estimation, the first step is to compute the Discrete Fourier Transform (DFT) using one fundamental frequency data. The time interval between two DFT is determined by the frequency estimate rate. Assuming the frequency is computed every quarter cycle (1/240 seconds), then the DFT needs to be computed every quarter cycle. For each DFT, it can be expressed by

$$
\left\{\begin{array}{l}
X_{r}=\frac{1}{\sqrt{2}} \frac{2}{N} \sum_{k=0}^{N-1} x_{k} \cos \left(\frac{2 \pi k}{N}\right) \\
X_{i}=\frac{1}{\sqrt{2}} \frac{2}{N} \sum_{k=0}^{N-1} x_{k} \sin \left(-\frac{2 \pi k}{N}\right)
\end{array}\right.
$$

The cos form and sin form in (13), together $\frac{1}{\sqrt{2}} \frac{2}{N}$ are constants, so they can be computed offline and saved in the memory of microprocessor. Therefore, the number of “*”" operation and '+' operation for $X_{r}$ and $X_{i}$ is $N$, and $(N-1)$, respectively.

With $X_{i}$ and $X_{r}$, the phase angle can be computed by

$$
\varphi=\arctan \left(\frac{X_{i}}{X_{r}}\right)
$$

As frequency is the derivate of phase angle, then we have 


$$
\varphi^{\prime}(m)=\frac{\varphi(m)-\varphi(m-1)}{\Delta t}=2 \pi f(m), m \geq 1
$$

where $m$ is the sequence index of phase angle, $f(m)$ is the estimated grid frequency, and $\Delta t$ is the time interval between two phase angles. According to (15), the frequency can be computed by $\varphi(m)$ and $\varphi(m-1)$. However, the power grid waveforms are always distorted by a variety of disturbances such as noise, harmonics, oscillation, etc. Therefore, in practice, usually a series of phase angles are used for the frequency estimation.

First, assuming the number of phase angles used for one frequency estimation is $(2 L+1)$. A quadratic polynomial is used to fit these phase angles, and the quadratic polynomial is expressed by

$$
p(i)=\alpha_{0}+\alpha_{1} t(i)+\alpha_{2} t(i)^{2}, i=0, \ldots, 2 L
$$

where $\alpha_{0}, \alpha_{1}, \alpha_{2}$ are the coefficients of the quadratic polynomial, $t(i)=(i-L) \Delta t$.

The derivate of $p(i)$ can be expressed by

Further, at sample index $i=L$,

$$
p^{\prime}(i)=\alpha_{1}+2 \alpha_{2} t(i)
$$

$$
p^{\prime}(L)=\alpha_{1}
$$

Therefore, the frequency at sample index $i=L$ can be calculated by

$$
f=\frac{\alpha_{1}}{2 \pi}
$$

According to (19), we only need estimate $\alpha_{1}$ to estimate frequency. The curve fitting errors are minimized to estimate $\alpha_{1}$. Using the estimated phase angle $\varphi(i)$ and the fitting curve $p(i)$, the sum of the square of the error can be expressed by

$$
e=\epsilon\left(\alpha_{0}, \alpha_{1}, \alpha_{2}\right)=\sum_{0}^{2 L}(p(i)-\varphi(i))^{2} .
$$

To minimize the error $e$, the partial derivatives of $e$ with respect to the coefficient $\alpha_{0}, \alpha_{1}, \alpha_{2}$ should be equal to zero, respectively.

The equation resulting from evaluating the partial derivative of $e$ with respect to $\alpha_{0}, \alpha_{1}$, and $\alpha_{2}$ are presented as follows.

$$
\begin{gathered}
\frac{\partial e}{\partial \alpha_{0}}=2 \alpha_{0}(2 L+1)+2 \alpha_{1} \sum_{i=0}^{2 L} t(i)+2 \alpha_{2} \sum_{i=0}^{2 L} t(i)^{2}-2 \sum_{i=0}^{2 L} \varphi(i) \\
\frac{\partial e}{\partial \alpha_{1}}=2 \alpha_{0} \sum_{i=0}^{2 L} t(i)+2 \alpha_{1} \sum_{i=0}^{2 L} t(i)^{2}+2 \alpha_{2} \sum_{i=0}^{2 L} t(i)^{3}-2 \sum_{i=0}^{2 L} \varphi(i) t(i) \\
\frac{\partial e}{\partial \alpha_{2}}=2 \alpha_{0} \sum_{i=0}^{2 L} t(i)^{2}+2 \alpha_{1} \sum_{i=0}^{2 L} t(i)^{3}+2 \alpha_{2} \sum_{i=0}^{2 L} t(i)^{4}-2 \sum_{i=0}^{2 L} \varphi(i) t(i)^{2}
\end{gathered}
$$

To error $e$ is minimized when the derivatives are equal to zero (i.e., $\frac{\partial e}{\partial \alpha_{0}}=\frac{\partial e}{\partial \alpha_{1}}=\frac{\partial e}{\partial \alpha_{2}}=0$ ), thus, 


$$
\left[\begin{array}{lll}
(2 L+1) & \sum_{i=0}^{2 L} t(i) & \sum_{i=0}^{2 L} t(i)^{2} \\
\sum_{i=0}^{2 L} t(i) & \sum_{i=0}^{2 L} t(i)^{2} & \sum_{i=0}^{2 L} t(i)^{3} \\
\sum_{i=0}^{2 L} t(i)^{2} & \sum_{i=0}^{2 L} t(i)^{3} & \sum_{i=0}^{2 L} t(i)^{4}
\end{array}\right]\left[\begin{array}{l}
\alpha_{0} \\
\alpha_{1} \\
\alpha_{2}
\end{array}\right]=\left[\begin{array}{c}
\sum_{i=0}^{2 L} \varphi(i) \\
\sum_{i=0}^{2 L} \varphi(i) t(i) \\
\sum_{i=0}^{2 L} \varphi(i) t(i)^{2}
\end{array}\right] .
$$

Because $t(i)=(i-L) \Delta t$,

Therefore, (24) can be rewritten as

$$
\sum_{i=0}^{2 L} t(i)=\sum_{i=0}^{2 L} t(i)^{3}=0 .
$$

$$
\left[\begin{array}{ccc}
(2 L+1) & 0 & \sum_{i=0}^{2 L} t(i)^{2} \\
0 & \sum_{i=0}^{2 L} t(i)^{2} & 0 \\
\sum_{i=0}^{2 L} t(i)^{2} & 0 & \sum_{i=0}^{2 L} t(i)^{4}
\end{array}\right]\left[\begin{array}{l}
\alpha_{0} \\
\alpha_{1} \\
\alpha_{2}
\end{array}\right]=\left[\begin{array}{c}
\sum_{i=0}^{2 L} \varphi(i) \\
\sum_{i=0}^{2 L} \varphi(i) t(i) \\
\sum_{i=0}^{2 L} \varphi(i) t(i)^{2}
\end{array}\right]
$$

Simply the above equation as

$$
T \alpha=\varphi
$$

Then, applying $\boldsymbol{T}^{\mathbf{- 1}}$ on both sides yields:

where $\boldsymbol{T}^{-\mathbf{1}}$ is the inverse of matrix $\boldsymbol{T}$.

$$
\alpha=T^{-1} \varphi
$$

(28) can be expanded as

$$
\left[\begin{array}{l}
\alpha_{0} \\
\alpha_{1} \\
\alpha_{2}
\end{array}\right]=\left[\begin{array}{lll}
T_{11} & T_{12} & T_{13} \\
T_{21} & T_{22} & T_{23} \\
T_{31} & T_{32} & T_{33}
\end{array}\right]\left[\begin{array}{l}
\sum_{i=0}^{2 L} \varphi(i) \\
\sum_{i=0}^{2 L} \varphi(i) t(i) \\
\sum_{i=0}^{2 L} \varphi(i) t(i)^{2}
\end{array}\right]
$$

where $T_{i j}$ is the entry of matrix $\boldsymbol{T}^{-\mathbf{1}}$.

Because only $\alpha_{1}$ is needed for frequency estimation, only $T_{21}, T_{22}$, and $T_{23}$ in $\boldsymbol{T}^{-1}$ need to be calculated, which are as follows:

$$
T_{21}=-\frac{1}{\operatorname{det}\left(T^{-1}\right)}\left|\begin{array}{cc}
0 & \sum_{i=0}^{2 L} t(i)^{2} \\
0 & \sum_{i=0}^{2 L} t(i)^{4}
\end{array}\right|=0
$$




$$
\begin{aligned}
T_{22} & =\frac{1}{\operatorname{det}\left(T^{-1}\right)}\left|\begin{array}{cc}
2 L+1 & \sum_{i=0}^{2 L} t(i)^{2} \\
\sum_{i=0}^{2 L} t(i)^{2} & \sum_{i=0}^{2 L} t(i)^{4}
\end{array}\right| \\
& =\frac{(2 L+1) \sum_{i=0}^{2 L} t(i)^{4}-\left(\sum_{i=0}^{2 L} t(i)^{2}\right)^{2}}{\operatorname{det}\left(\boldsymbol{T}^{-1}\right)} \\
T_{23} & =-\frac{1}{\operatorname{det}\left(\boldsymbol{T}^{-1}\right)}\left|\begin{array}{cc}
2 L+1 & 0 \\
\sum_{i=0}^{2 L} t(i)^{2} & 0
\end{array}\right|=0
\end{aligned}
$$

where $\operatorname{det}\left(\boldsymbol{T}^{-\mathbf{1}}\right)$ is the determinant of $\boldsymbol{T}^{-\mathbf{1}}$.

Since $T_{21}=T_{23}=0, \alpha_{1}$ can be expressed by

$$
\begin{aligned}
& \alpha_{1}=T_{21} \sum_{i=0}^{2 L} \varphi(i)+T_{22} \sum_{i=0}^{2 L} \varphi(i) t(i)+T_{23} \sum_{i=0}^{2 L} \varphi(i) t(i)^{2} \\
& =T_{22} \sum_{i=0}^{2 L} \varphi(i) t(i) .
\end{aligned}
$$

Note that $T_{22}$ only depends on the parameters of $L$ and $\Delta t$, which are constant parameters, $T_{22} * t(i)$ can be calculated offline and saved in the memory of microprocessor.

Typically, using (19) and (33), a new frequency can be estimated using a total number of $(2 L+1)$ phase angles. We can conclude that the computation time for a new frequency estimation is proportional to $L$ since it requires $(2 L+1)$ multiplications and $2 L$ additions according to (33).

In practice, because the phase angle obtained by (14) is between $-\pi$ to $\pi$. Thus, the angles may need to be unwrapped for least squares fitting. The example code of unwrapping is provided below.

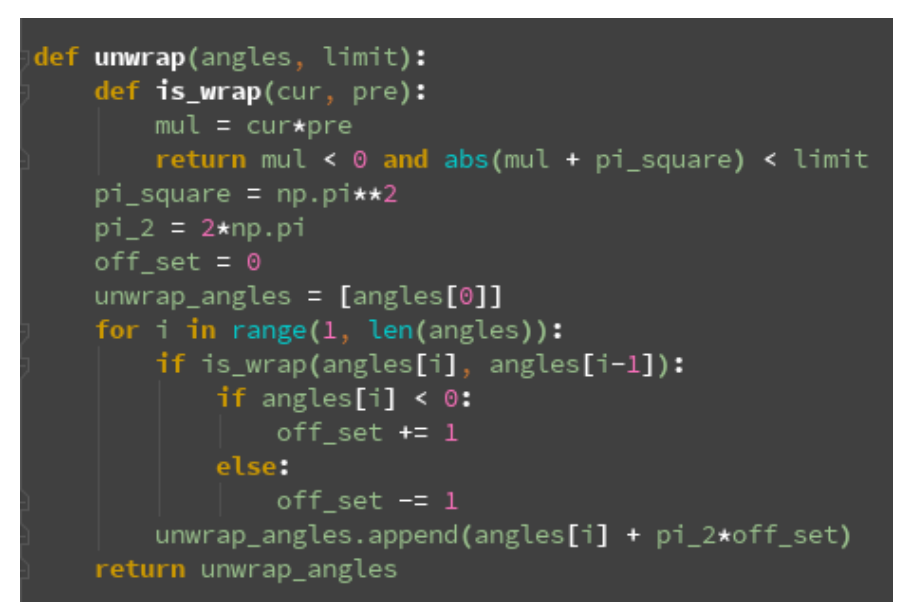

Table $\mathrm{X}$ summarizes the count of numerical operations for each part. The total numerical operation is equal to $(4 \mathrm{~N}+22 \mathrm{~L}+1)$. Assuming the sampling rate is $5760 \mathrm{~Hz}$ (i.e., $\mathrm{N}=96$ ), and the $\mathrm{L}$ is equal to 9 , the total number of numerical operations is equal to 295 . 
Table X Count of numerical operations of frequency estimator

\begin{tabular}{|c|c|c|c|c|c|c|}
\hline & $\begin{array}{l}\text { count of “+”" } \\
\text { and "_-" }\end{array}$ & count of “*” & count of “"” & $\begin{array}{l}\text { count of } \\
\text { 'abs' }\end{array}$ & $\begin{array}{l}\text { count of } \\
\text { '>' or ' }<\text { ' }\end{array}$ & $\begin{array}{l}\text { count of } \\
\text { "arctan" }\end{array}$ \\
\hline $\begin{array}{c}\text { Angle computation } \\
\text { by DFT }\end{array}$ & $2(\mathrm{~N}-1)$ & $2 \mathrm{~N}$ & 0 & 0 & 0 & 1 \\
\hline $\begin{array}{l}\text { Phase Angle } \\
\text { Unwrapping }\end{array}$ & $6 \mathrm{~L}$ & $4 \mathrm{~L}$ & 0 & $2 \mathrm{~L}$ & $6 \mathrm{~L}$ & 0 \\
\hline $\begin{array}{c}\text { Frequency } \\
\text { computation by } \\
\text { Least Squares } \\
\text { Fitting - Polynomial }\end{array}$ & $2 \mathrm{~L}$ & $2 \mathrm{~L}+1$ & 1 & 0 & 0 & 0 \\
\hline Total & $2(\mathrm{~N}-1)+8 \mathrm{~L}$ & $2 \mathrm{~N}+6 \mathrm{~L}+1$ & 1 & $2 \mathrm{~L}$ & $6 \mathrm{~L}$ & 1 \\
\hline
\end{tabular}

\subsubsection{Computation Time Analysis of the Frequency Estimator on Digital Signal Processor}

Similar as the analysis of the transient detector, Table XI shows the computation utilization rate of the frequency estimation on the DSP. The rate is only $0.05 \%$, which is very low. It means that the computational cos of the frequency estimator is very low for a modern DSP.

Table XI Computation utilization rate of the frequency estimator

\begin{tabular}{|l|c|c|c|c|c|}
\hline & $\begin{array}{c}\text { \# of numerical } \\
\text { operations per } \\
\text { measurement }\end{array}$ & $\begin{array}{c}\text { \# of measurements } \\
\text { per second }\end{array}$ & $\begin{array}{c}\text { Total \# of } \\
\text { numerical } \\
\text { operations } \\
\text { per second }\end{array}$ & $\begin{array}{c}\text { MFLOPS of the } \\
\text { DSP }\end{array}$ & $\begin{array}{c}\text { Computation } \\
\text { Utilization Rate }\end{array}$ \\
\hline $\begin{array}{c}\text { Frequency } \\
\text { Estimation }\end{array}$ & 295 & 240 & 70,800 & $150,000,000$ & $0.05 \%$ \\
\hline
\end{tabular}

\subsubsection{Computation Time Simulation Test of The Frequency Estimator}

The computation time of the frequency estimator has also been evaluated on a $3.6 \mathrm{GHz} 4$-core microprocessor.

Different combinations of sampling rate, window length and time of test signal have been evaluated to perform the computation time testing. The testing results are shown in Table XII, Table XIII, and Table XIV. For a specific sampling rate, the frequency computation time is mainly determined by the window length. The computation time for per frequency calculation increases by about 10 microseconds from a shorter window ( 2.5 cycle) to a typical window size $(5.5$ cycle). The computation time increase is trivial. However, a longer window can contribute to better noise rejection performance. Table XV, Table XVI, and Table XVII show the frequency measurement errors under different sampling rate and window size. It could be seen that compared to the sampling rate, a longer window size is preferred to mitigate the measurement errors. At the sampling rate of $1440 \mathrm{~Hz}$ and window size of 5.5-cycle, the maximum frequency measurement error under $60 \mathrm{~dB}$ noise is below 2 Millihertz $(0.002 \mathrm{~Hz})$, which is accurate enough for practical applications. In this case, the computation time is about 40 microseconds $(0.00004$ seconds) for a frequency computation. Note that the frequency measure rate is a quarter cycle, that is $1 / 240$ second for a $60 \mathrm{~Hz}$ power grid. It means we estimate a frequency every $1 / 240$ second. 
Table XII Computation time test of the frequency estimator $($ sampling rate $=1440 \mathrm{~Hz}$ )

\begin{tabular}{|l|l|l|l|l|}
\hline \multirow{2}{*}{$\begin{array}{l}\text { Sampling } \\
\text { Rate }\end{array}$} & \multirow{2}{*}{$\begin{array}{l}\text { Window } \\
\text { length(=L/2+1) }\end{array}$} & $\begin{array}{l}\text { Time of test } \\
\text { signal }\end{array}$ & Computation Time \\
\cline { 3 - 5 } & \multirow{2}{*}{2.5} & 1 & Total (Second) & $\begin{array}{l}\text { Per calculation } \\
\text { (Microsecond) }\end{array}$ \\
\hline & & 10 & 0.007 & 31 \\
\hline \multirow{2}{*}{$1440 \mathrm{~Hz}$} & \multirow{2}{*}{4} & 100 & 0.072 & 30 \\
\hline & & 1 & 0.76 & 32 \\
\hline & \multirow{2}{*}{5.5} & 10 & 0.008 & 36 \\
\hline & & 100 & 0.083 & 35 \\
\hline & & 10 & 0.868 & 36 \\
\hline & & 100 & 0.009 & 42 \\
\hline
\end{tabular}

Table XIII Computation time test of the frequency estimator $($ sampling rate $=2880 \mathrm{~Hz}$ )

\begin{tabular}{|l|l|l|l|l|}
\hline \multirow{2}{*}{$\begin{array}{l}\text { Sampling } \\
\text { Rate }\end{array}$} & $\begin{array}{l}\text { Window } \\
\text { length(=L/2+1) }\end{array}$ & $\begin{array}{l}\text { Time of test } \\
\text { signal }\end{array}$ & \multicolumn{2}{|l|}{ Computation Time } \\
\cline { 3 - 5 } & \multirow{2}{*}{2.5} & 1 & Total (Seconds) & $\begin{array}{l}\text { Per calculation } \\
\text { (Microsecond) }\end{array}$ \\
\hline & & 10 & 0.011 & 48 \\
\hline \multirow{2}{*}{$2880 \mathrm{~Hz}$} & \multirow{2}{*}{4} & 100 & 0.112 & 47 \\
\hline & & 1 & 1.12 & 47 \\
\hline & \multirow{2}{*}{5.5} & 10 & 0.012 & 54 \\
\hline & & 100 & 0.122 & 51 \\
\hline & & 10 & 1.23 & 51 \\
\hline & & 100 & 0.012 & 55 \\
\hline
\end{tabular}

Table XIV Computation time test of the frequency estimator (sampling rate $=5760 \mathrm{~Hz}$ )

\begin{tabular}{|c|c|c|c|c|}
\hline \multirow{2}{*}{$\begin{array}{l}\text { Sampling } \\
\text { Rate }\end{array}$} & \multirow{2}{*}{$\begin{array}{l}\text { Window } \\
\text { length }(=\mathrm{L} / 2+1)\end{array}$} & \multirow{2}{*}{$\begin{array}{l}\text { Time of test } \\
\text { signal }\end{array}$} & \multicolumn{2}{|c|}{ Computation Time } \\
\hline & & & Total (Second) & $\begin{array}{l}\text { Per calculation } \\
\text { (Microsecond) }\end{array}$ \\
\hline \multirow{9}{*}{$5760 \mathrm{~Hz}$} & \multirow{3}{*}{2.5} & 1 & 0.019 & 83 \\
\hline & & 10 & 0.191 & 80 \\
\hline & & 100 & 1.922 & 80 \\
\hline & \multirow{3}{*}{4} & 1 & 0.019 & 85 \\
\hline & & 10 & 0.201 & 84 \\
\hline & & 100 & 2.081 & 87 \\
\hline & \multirow{3}{*}{5.5} & 1 & 0.020 & 92 \\
\hline & & 10 & 0.213 & 89 \\
\hline & & 100 & 2.115 & 88 \\
\hline
\end{tabular}


Table XV Frequency measurement errors vs window size (sampling rate $=1440 \mathrm{~Hz}$ )

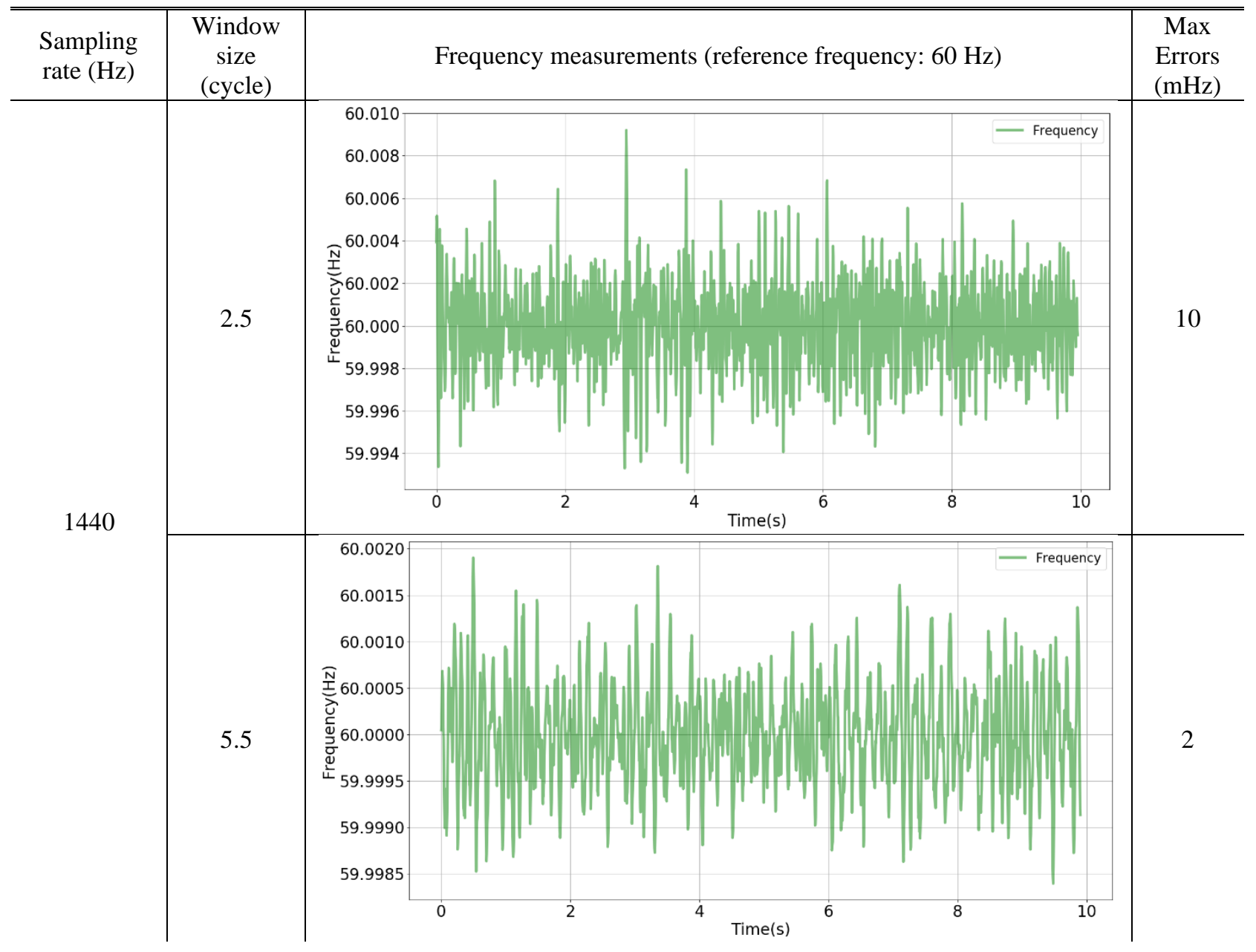


Table XVI Frequency measurement errors vs window size (sampling rate $=2880 \mathrm{~Hz}$ )

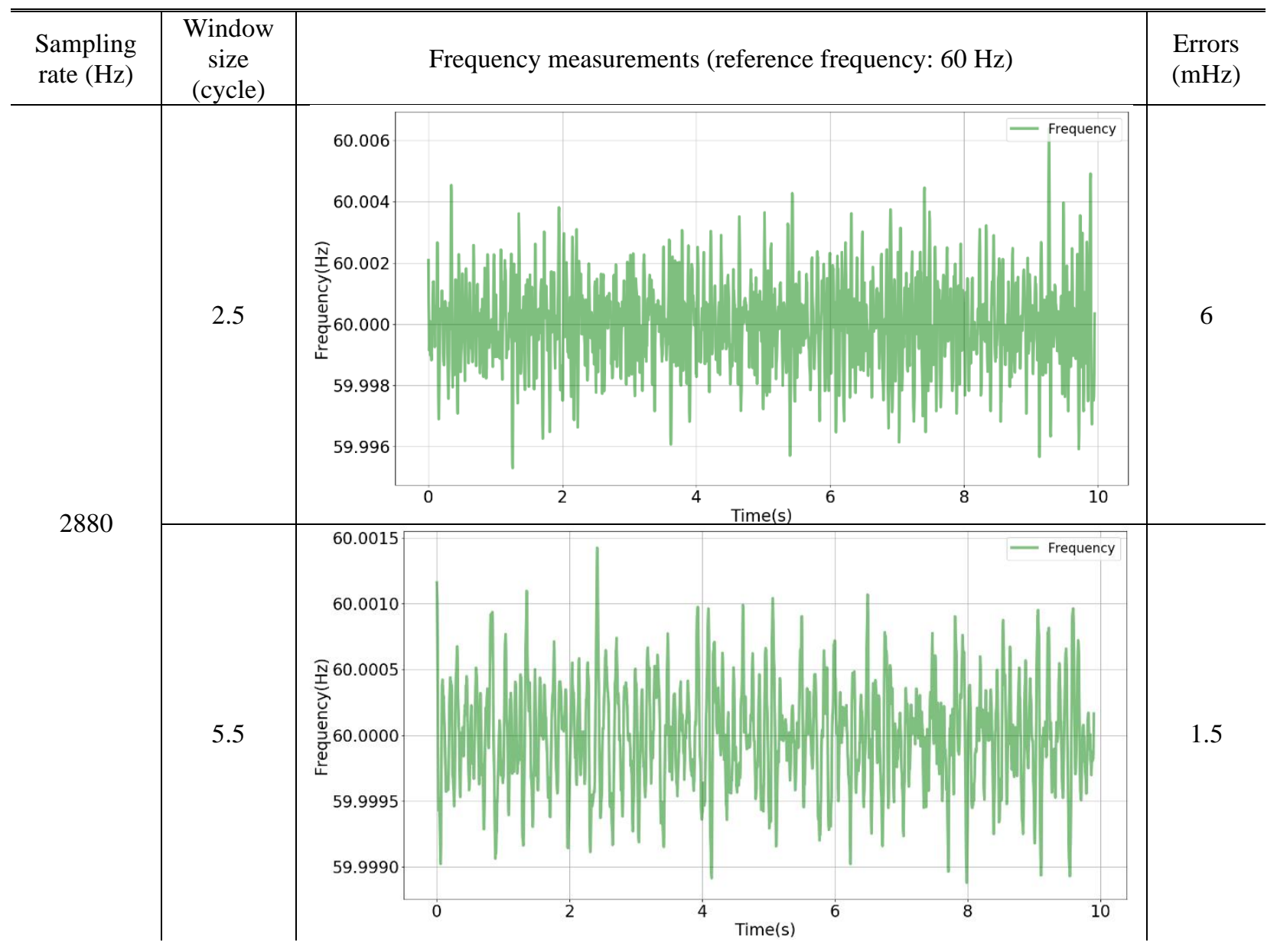


Table XVII Frequency measurement errors vs window size (sampling rate $=5760 \mathrm{~Hz}$ )

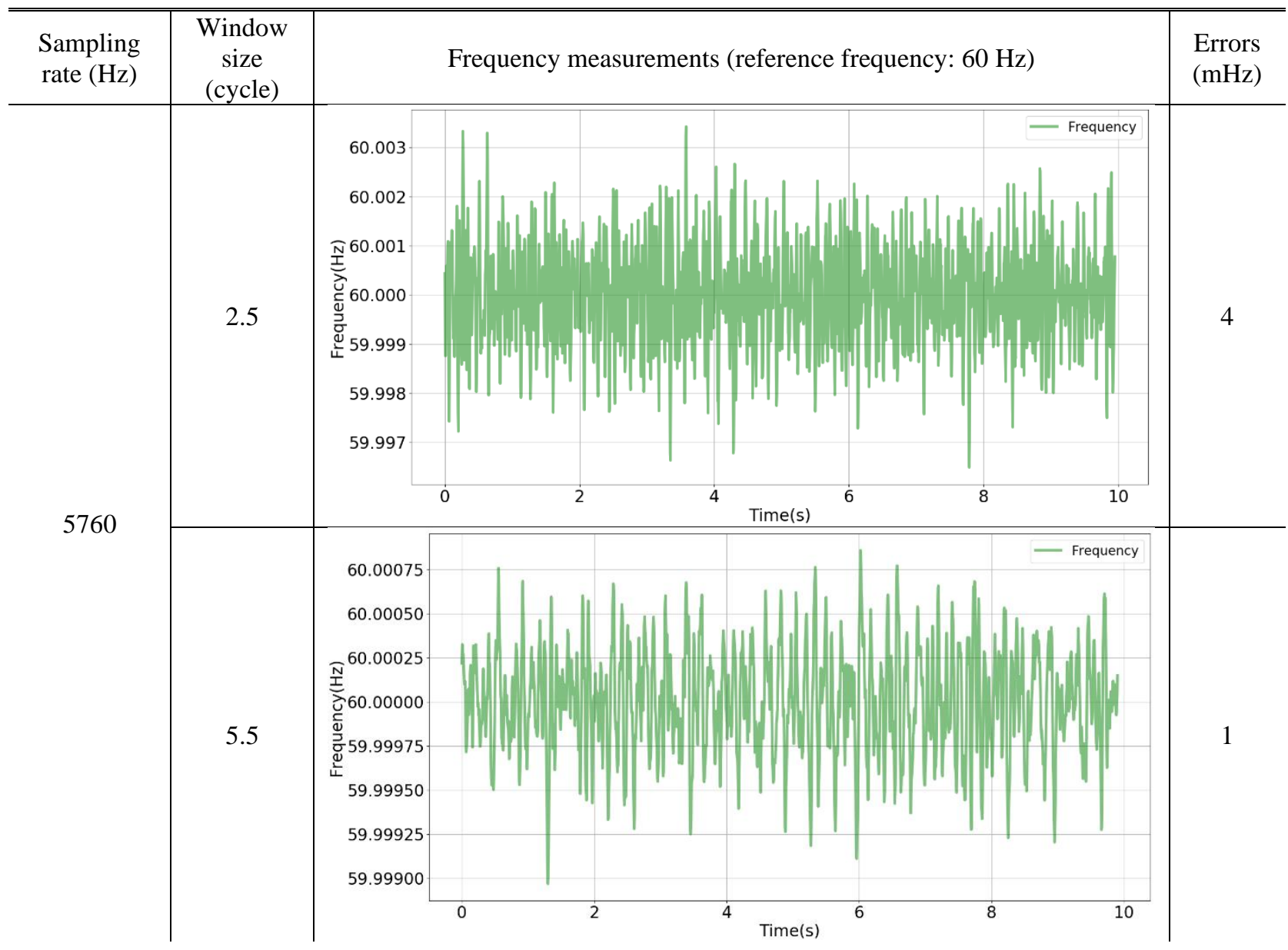

\subsection{HARDWARE VALIDATION}

In this section, the proposed fault-tolerant frequency measurement algorithm was successfully realized on a low-cost hardware, and it can work in real-time measurement. To verify the effectiveness and low computation cost of the algorithm, the algorithm has been implemented on hardware to demonstrate its feasibility. For the transient detector, the core of the algorithm, low-cost hardware and microprocessor are used to verify its low computational cost, and the microprocessor is an ARM $80 \mathrm{MHz}$ MCU. A series of testing waveforms were applied to test the performance. The results showed that during a fault period, the frequency measurement output had much small deviation from the steady state output. In this section, the results obtained from the test experiments were provided.

\subsubsection{Experiment setup for fault-tolerant frequency measurement}

The developed measuring system in the project included the fault detector and a tablet with an app to perform frequency computation and visualization. The fault detector sampled the voltage signal with high-precision GPS timing synchronization and performed transient event detection. At the same time, the fault detector transferred the sample data and fault detection result to the tablet. The frequency computation app running on the tablet processed the received data to determine whether it was necessary to trigger frequency lock and the adaptive frequency computation. In addition to the displaying on the tablet, an oscilloscope was used as a comparison for displaying the output waveform of the calibrator. 
Figure 86 presented the experiment setup for testing the developed frequency measurement and visualization system. The testing power signal was generated from the Fluke 6135A/PMUCAL PMU Calibration system, which is the only fully automated and traceable PMU calibration system available today.

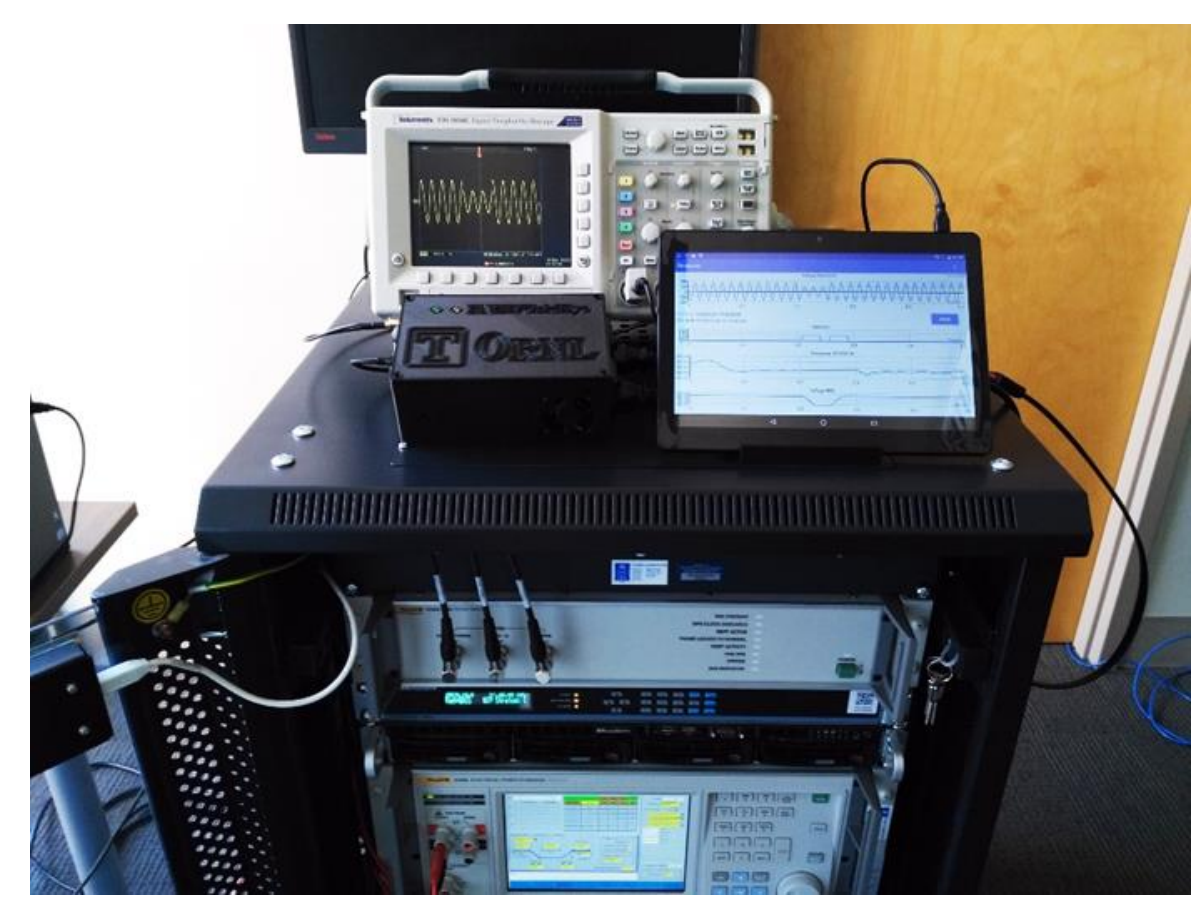

Figure 86 Experiment setup for fault-tolerant frequency measurement

\subsubsection{Hardware testing}

\subsubsection{Frequency measurement tests under normal grid operation}

\section{Connected to power grid}

In this experiment, the measuring system was connected to the power grid via $120-\mathrm{V}$ power outlet in laboratory. In this way, the real power grid data was feed into the measuring system. The measurement results are shown in Figure 87. As there is no system transient occurred, the computed frequency and RMS value of the PMU calibrator is very stable and fluctuate in a small range. The transient detection is 0 over the testing, and it demonstrated that the transient detector will not be mistakenly triggered by the normal grid waveform distortions such as noise, harmonics, etc. The small measurement variation could verify the frequency measurements are very stable in real-grid measurement environment and have good grid disturbance rejection performance. 


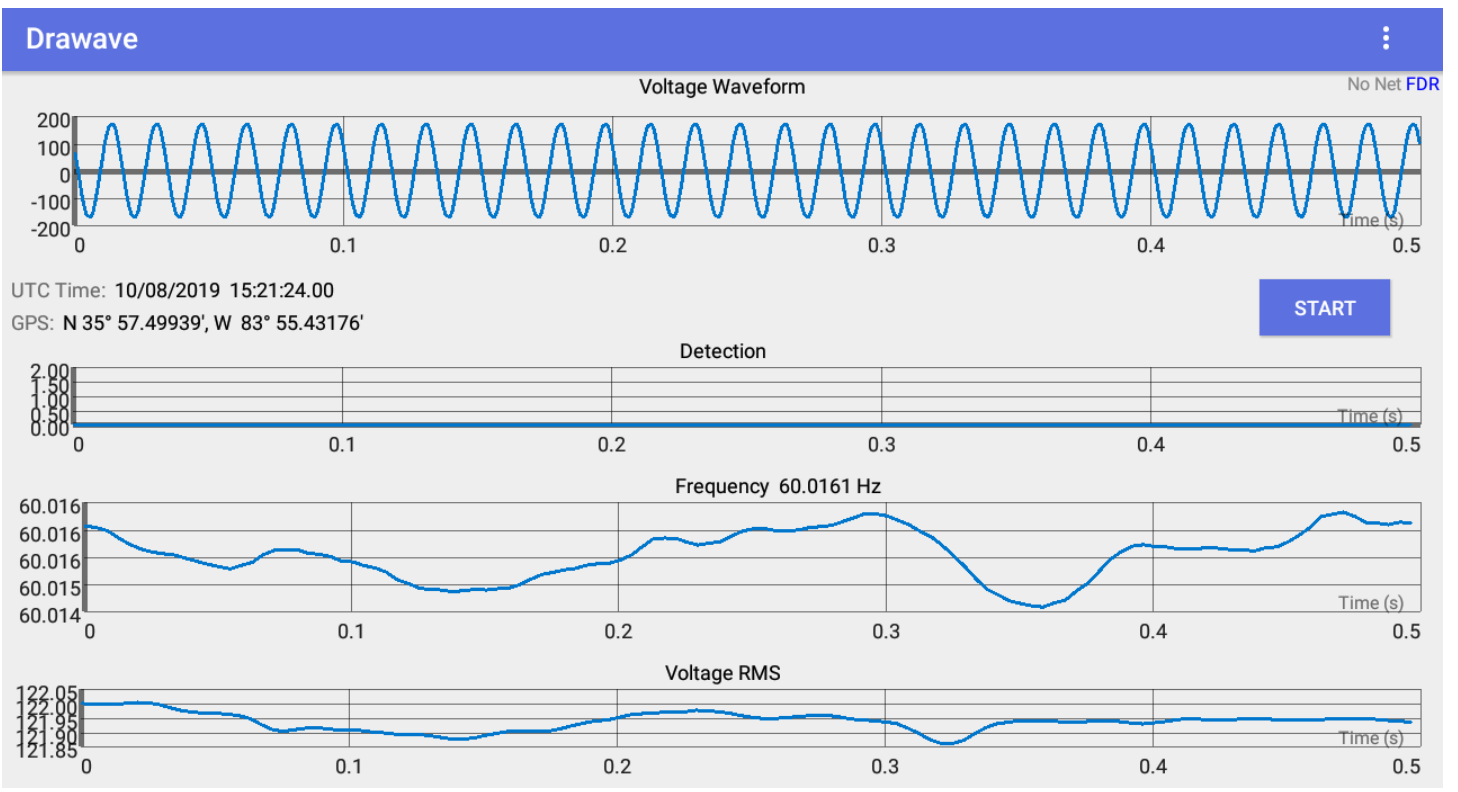

Figure 87 Real-time measurements of power grid (via 120-V power outlet)

\section{Connected to PMU calibrator}

In this test, the measuring system was connected to the PMU calibrator, and $60 \mathrm{~Hz}$ clean signal was generated from the PMU calibrator and used for the measuring system. The testing results are shown in Figure 88 . The frequency measurement is very stable and accurate. This test demonstrated the highprecision measurement accuracy of the developed algorithm, even using low-cost hardware.

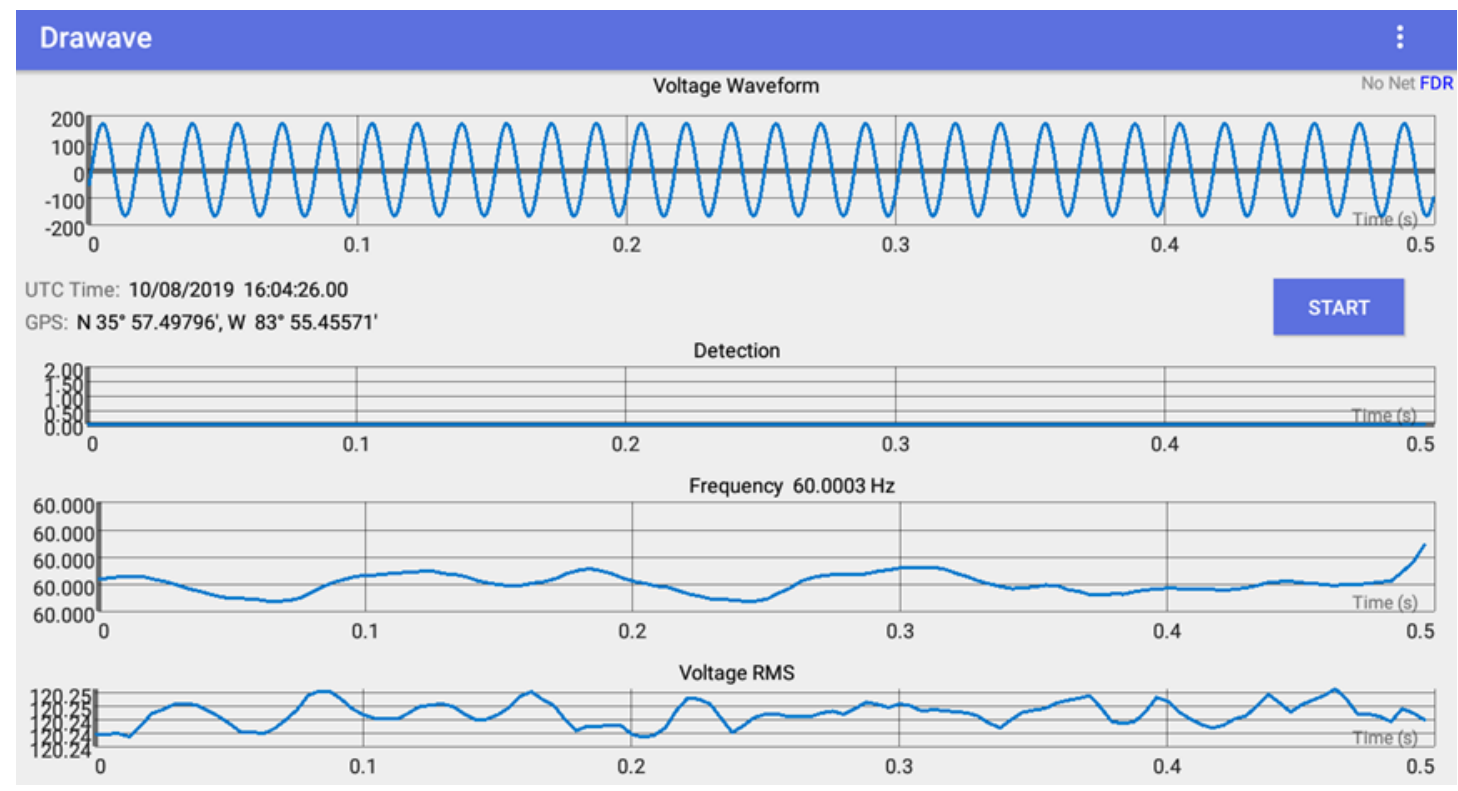

Figure 88 Real-time measurements of PMU Calibrator $(60 \mathrm{~Hz}$ clean test signal)

\subsubsection{Frequency measurement tests under voltage step change}

The experiments utilized the Dip function of the calibrator which created repeated dips in the output voltage waveform according to the settings. In the experiments, the waveform repeat period was set to 0.5 
$\mathrm{s}$ and in the middle of this period a voltage dip was applied for $0.05 \mathrm{~s}$. In the app, the displaying time range was $0.5 \mathrm{~s}$ and all curves were refreshed every $0.5 \mathrm{~s}$.

Experiments were performed on voltage sags with amplitude reduced to $10 \%$ to $90 \%$ of the normal voltage amplitude. Figure 89, Figure 90, and Figure 91 show the sag to 70\%, 50\%, and $20 \%$ magnitude, respectively. It could be seen that the transient detector detects the voltage step change event, and the frequency estimator also responses to the transient event. Although we can still see the frequency deviations during the transient, the deviation is very small, far away from the frequency triggering threshold (i.e., $57 \mathrm{~Hz}$ for low frequency tripping, and $61 \mathrm{~Hz}$ for high frequency tripping).

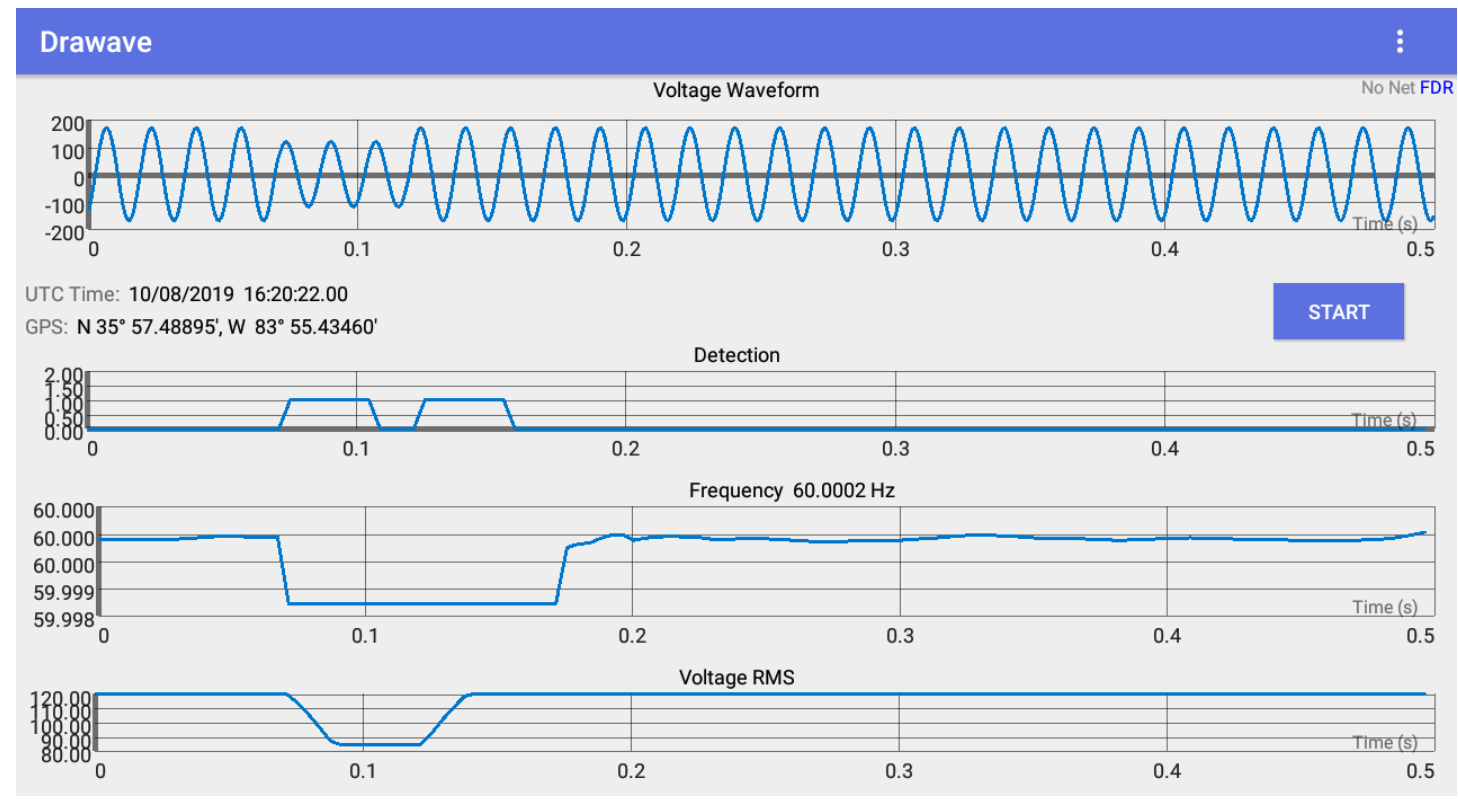

Figure 89 Real-time measurement result under sag to $70 \%$ amplitude

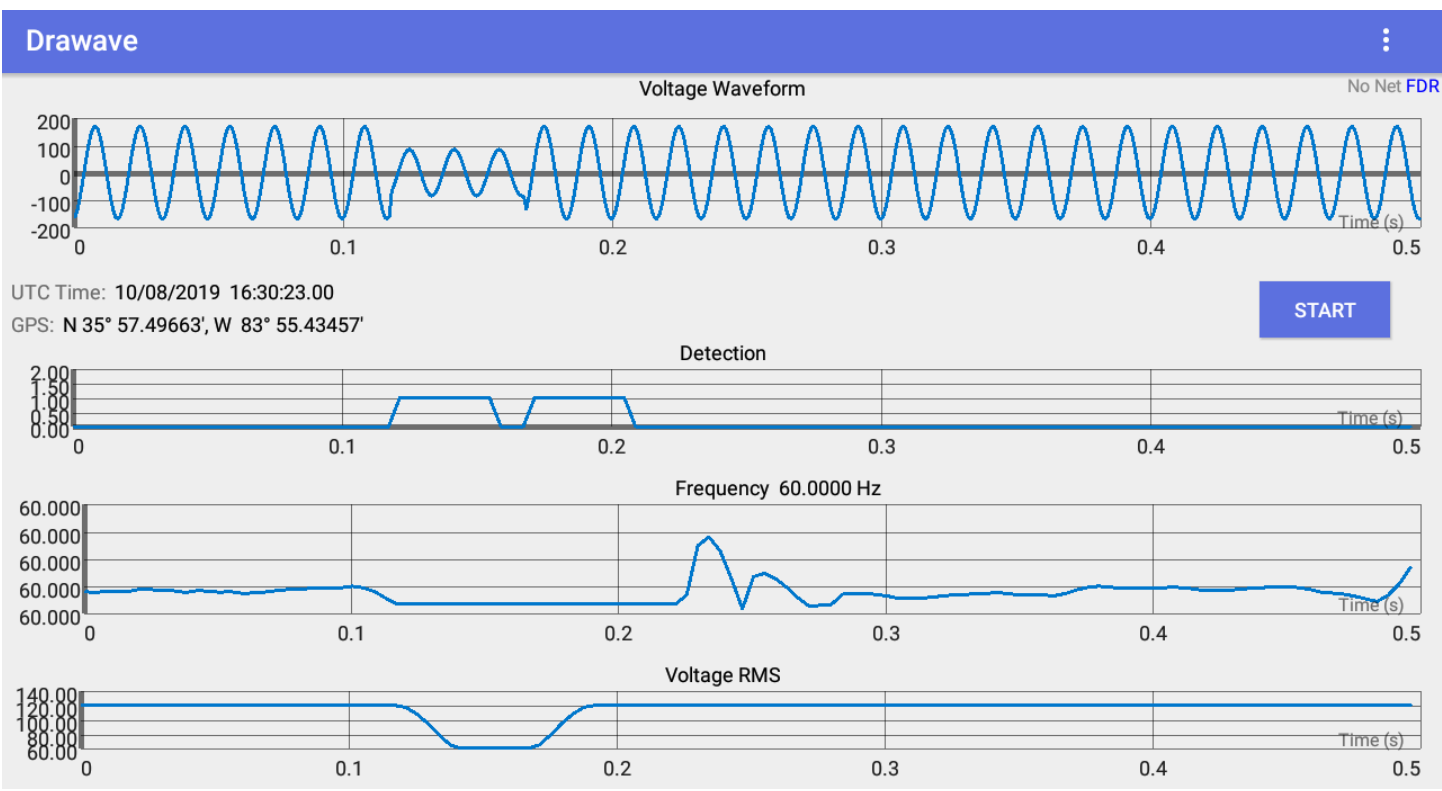

Figure 90 Real-time measurement result under sag to 50\% amplitude 


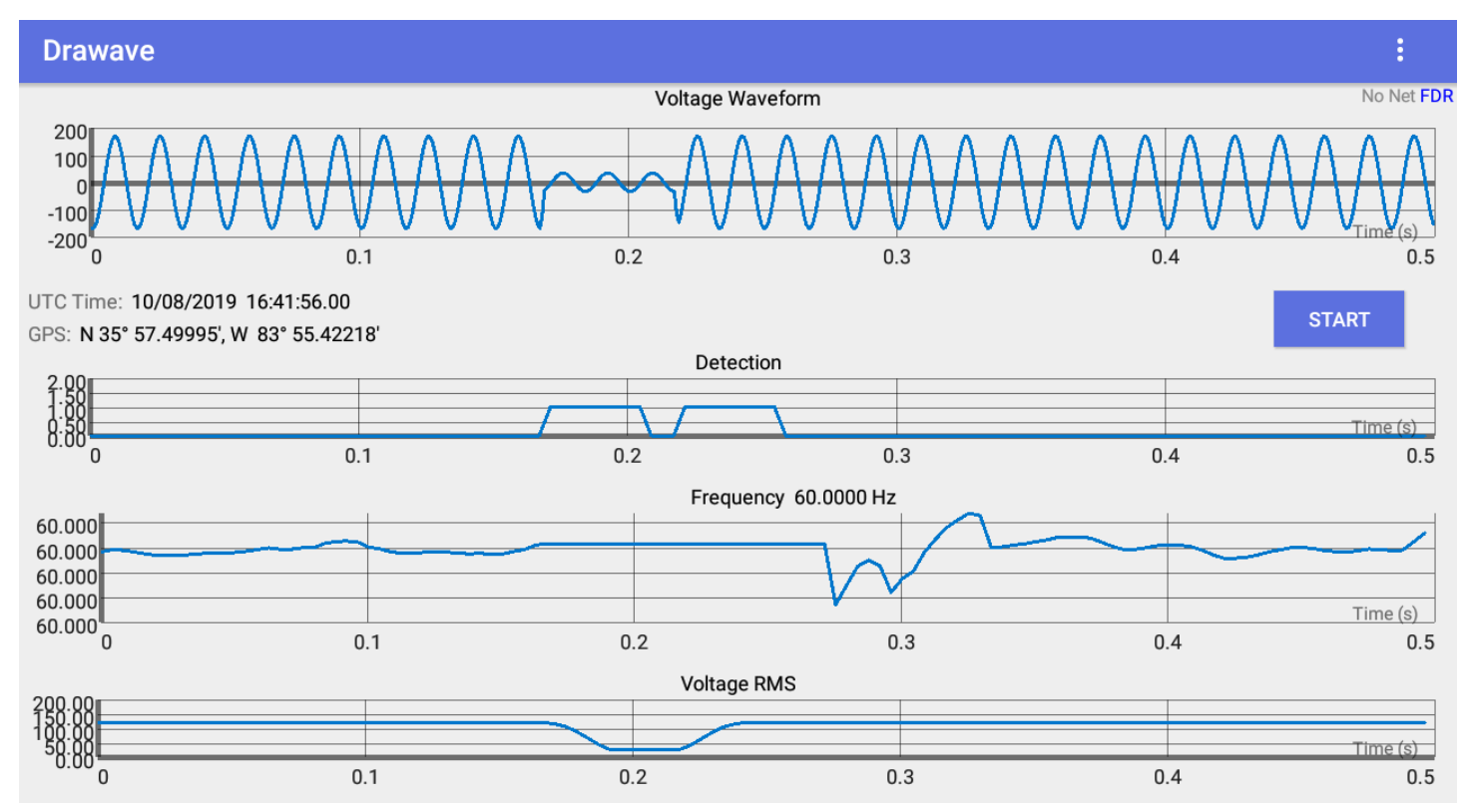

Figure 91 Real-time measurement result under sag to $20 \%$ amplitude

\subsubsection{Frequency measurement tests under phase angle jump}

To verify whether the developed fault-tolerant algorithm could have good performance during phase jump, experiments were conducted by using the phase jump waveforms with leading $60^{\circ}$ and lagging $60^{\circ}$, which were produced with IEEE 118-Bus transmission system. For each phase angle jump, the fault was first triggered at a position near the valley of the sinusoidal waveform, and then following faults were triggered at an interval of quarter cycle period. Totally eight phase angle jump waveforms were used in the experiments. And the results are presented from Figure 92 to Figure 99. Like previous results, the fault could be detected timely to perform frequency lock. After the frequency lock was released, the peaks appeared on the frequency curve indicated the adaptive process which increased window size when the waveform became stable gradually. The first frequency value was calculated in 2.5 cycles after the fault alarm was cleared. Then the estimation window will gradually increase to normal window size, and the frequency measurement error will decrease during this period. It should be noted that frequency spikes after the frequency lock release are also much smaller than the frequency triggering threshold (i.e. $57 \mathrm{~Hz}$ for low frequency tripping, and $61 \mathrm{~Hz}$ for high frequency tripping). These frequency spikes can be reduced by delaying the frequency measurements using a longer estimation window, but the disadvantage is the longer measurement latency. 


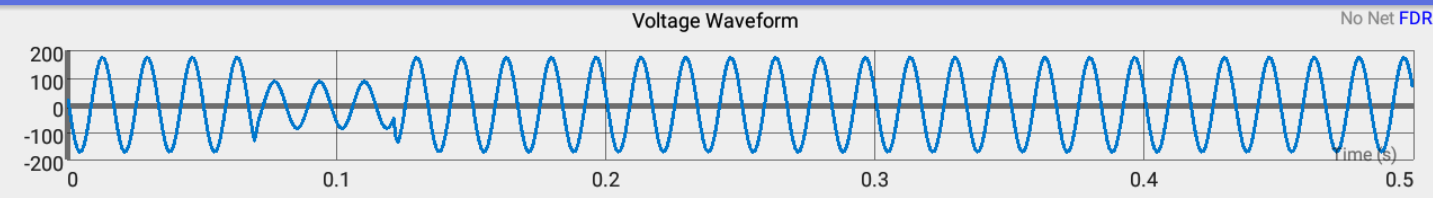

UTC Time: 10/08/2019 22:33:05.00

GPS: N $35^{\circ} 57.50603^{\prime}, W 83^{\circ} 55.45843^{\prime}$

START
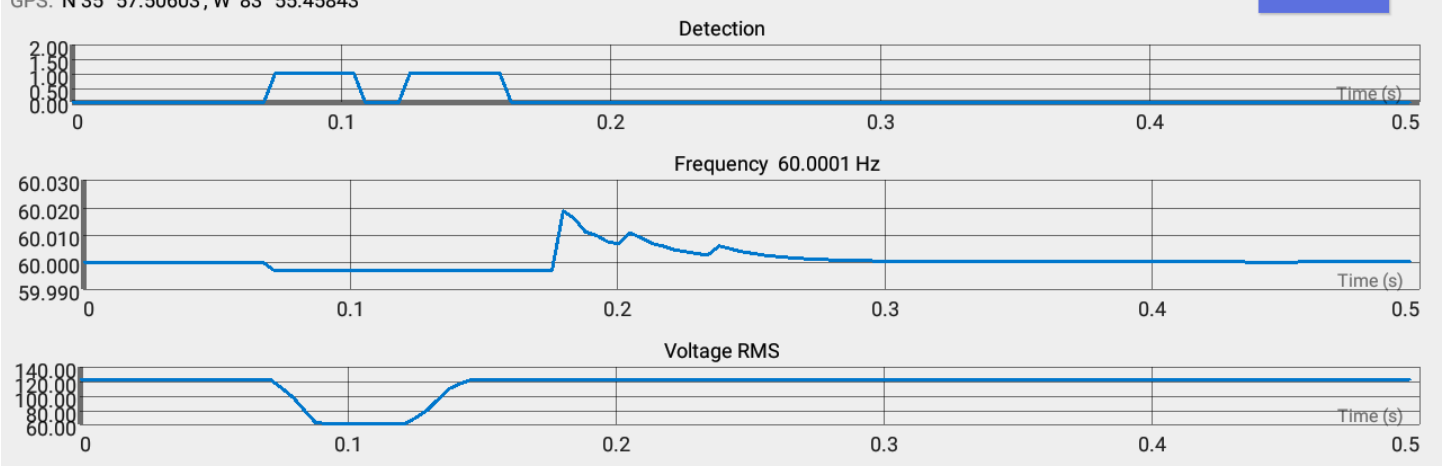

Figure 92 Real-time measurement result under 60-degree phase jump at 0/4 cycle

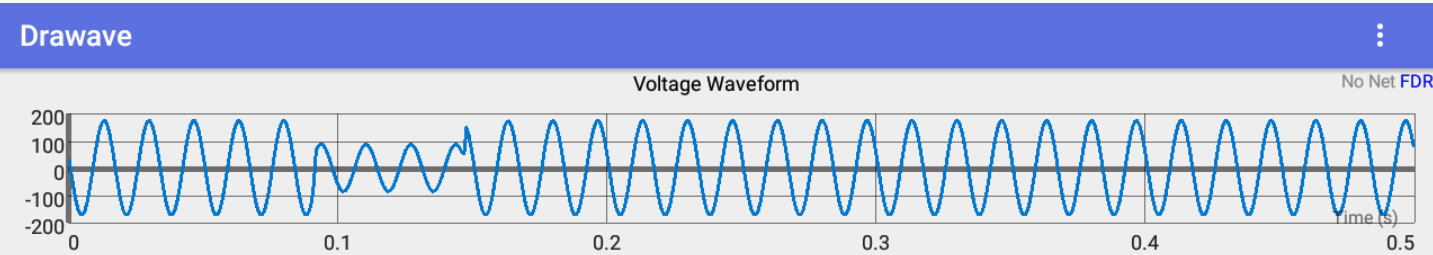

UTC Time: 10/08/2019 22:33:58.00

GPS: N $35^{\circ} 57.50819^{\prime}, \mathrm{W} 83^{\circ} 55.45476^{\prime}$
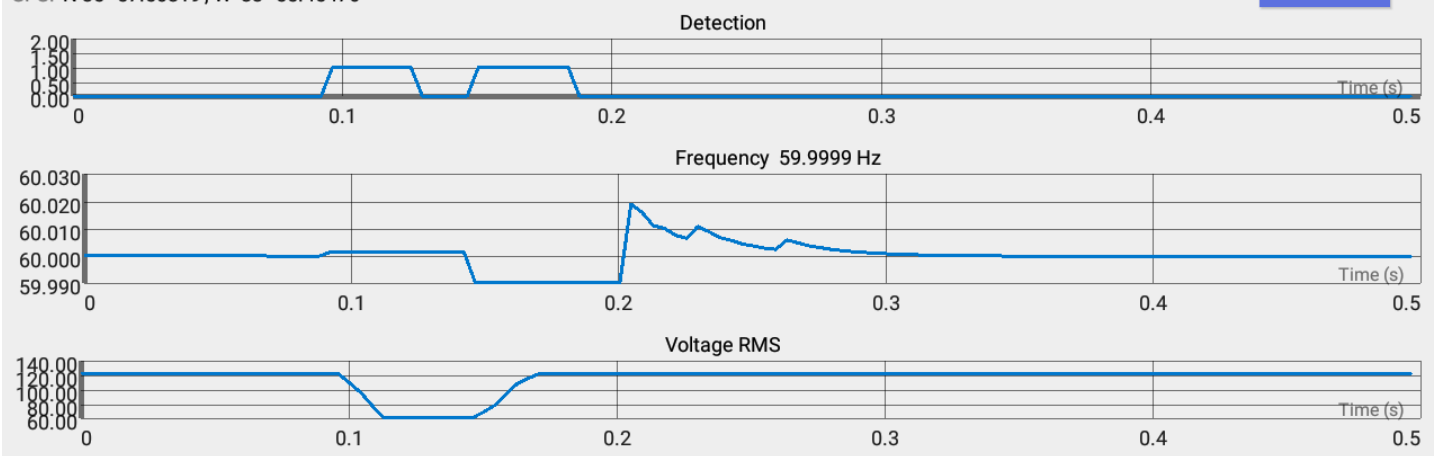

Figure 93 Real-time measurement result under 60-degree phase jump at 1/4 cycle 


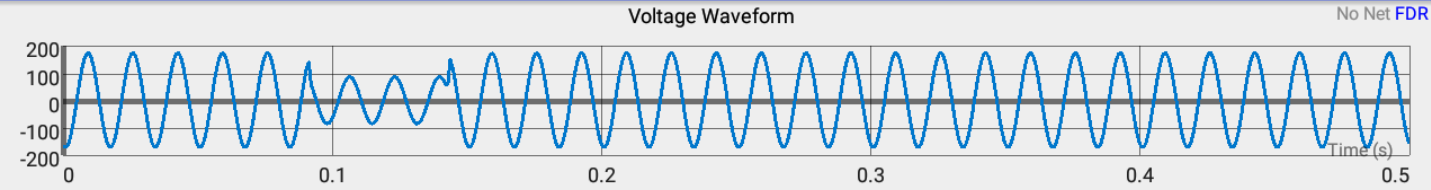

UTC Time: 10/08/2019 22:36:18.00

GPS: N $35^{\circ} 57.50960^{\prime}$, W $83^{\circ} 55.45515^{\prime}$

START
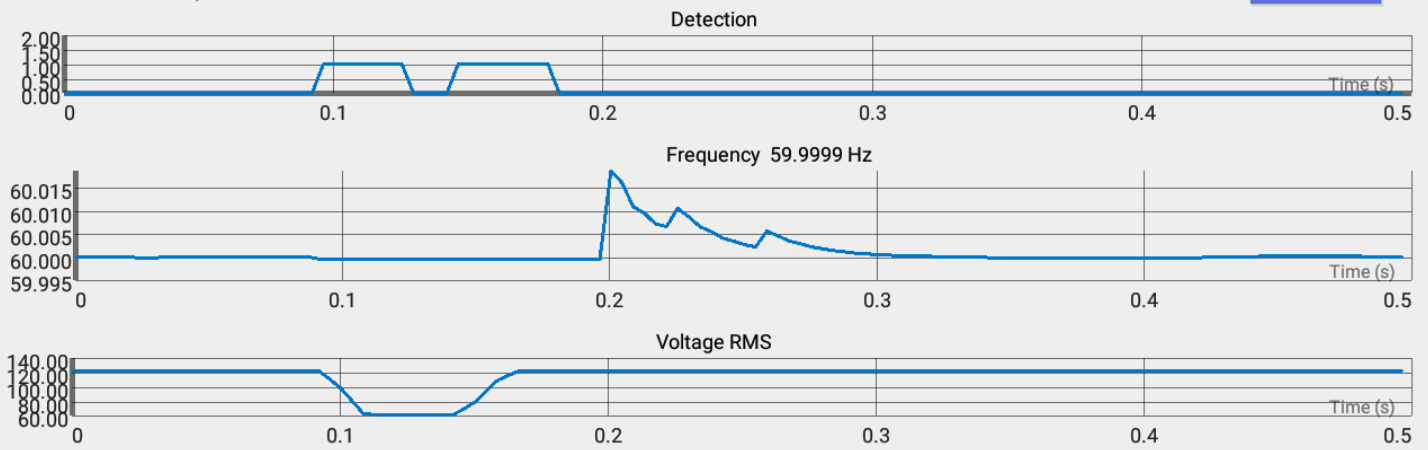

Figure 94 Real-time measurement result under 60-degree phase jump at 2/4 cycle

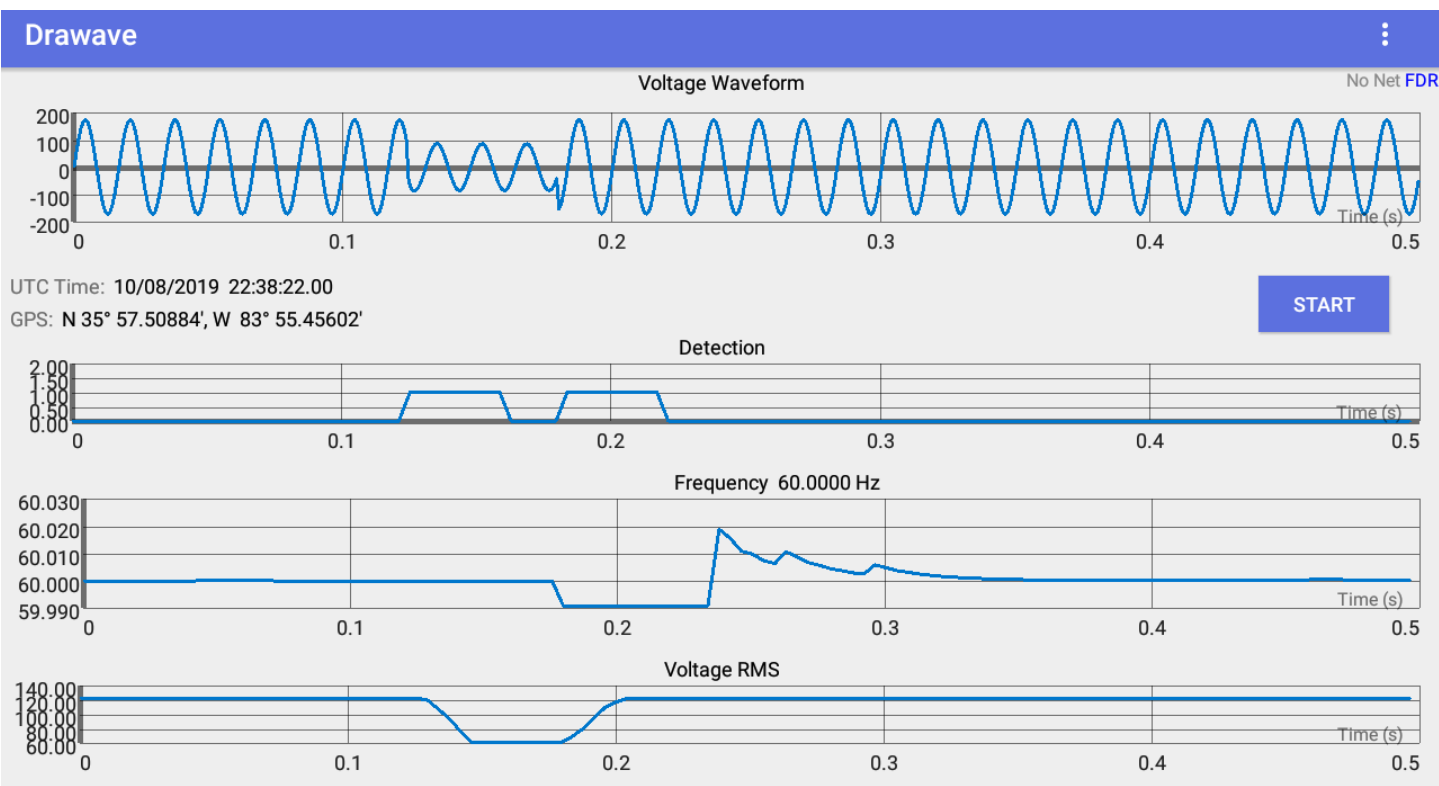

Figure 95 Real-time measurement result under 60-degree phase jump at 3/4 cycle 


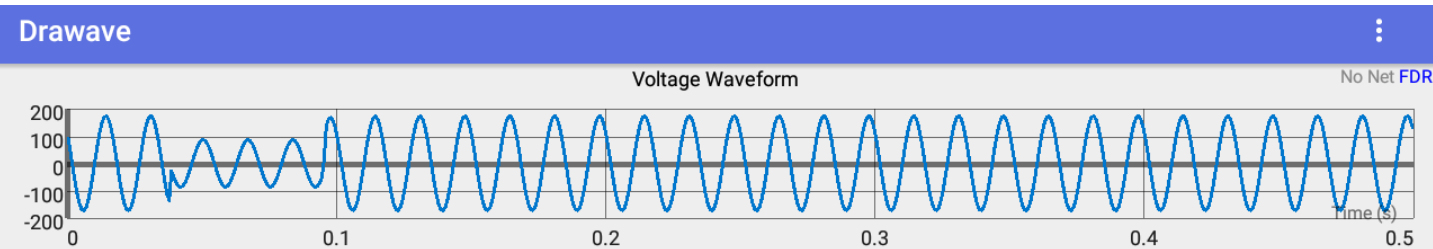

UTC Time: 10/08/2019 22:26:16.00

GPS: N $35^{\circ} 57.50874^{\prime}, \mathrm{W} 83^{\circ} 55.45873^{\prime}$

START
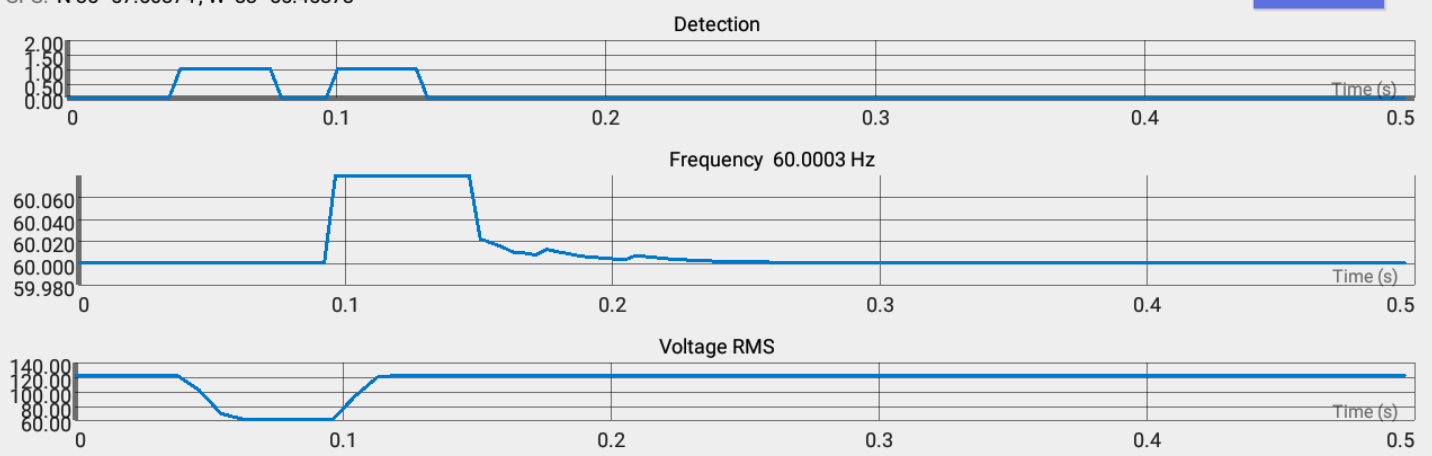

Figure 96 Real-time measurement result under -60-degree phase jump at 0/4 cycle

\section{Drawave}

Voltage Waveform

No Net FDR

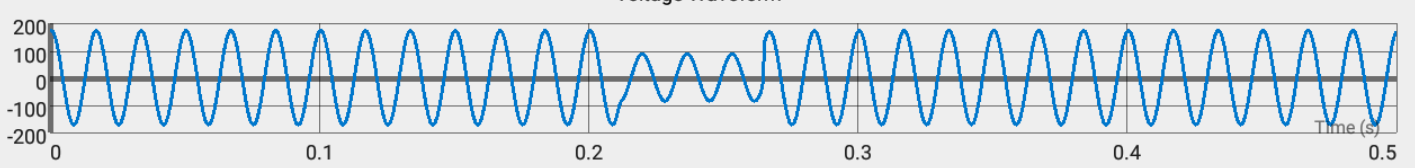

UTC Time: $10 / 08 / 2019$ 22:28:27.00

GPS: N $35^{\circ} 57.50964^{\prime}, \mathrm{W} 83^{\circ} 55.45919^{\prime}$

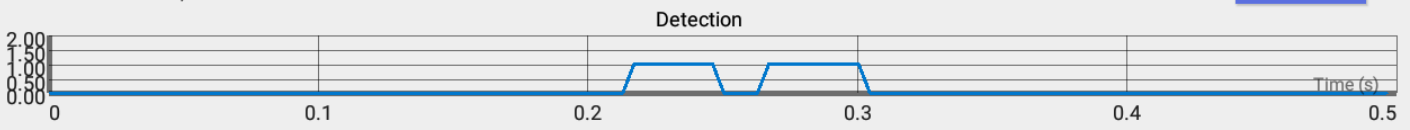

Frequency $59.9996 \mathrm{~Hz}$

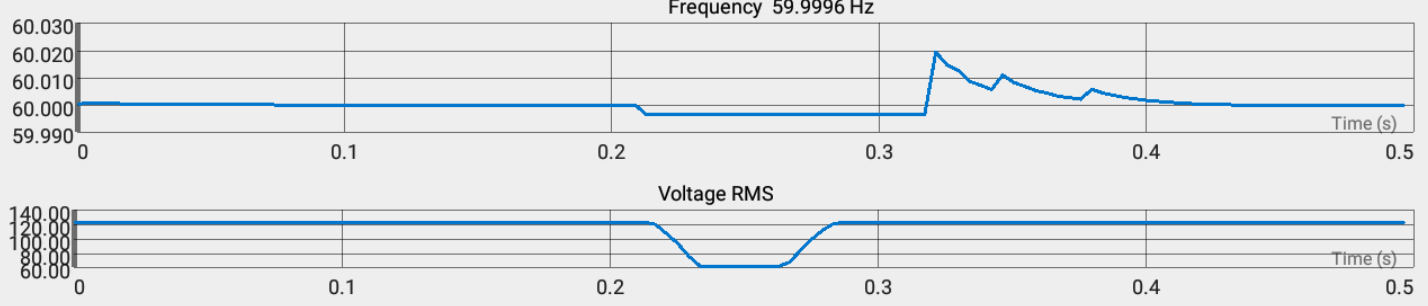

Figure 97 Real-time measurement result under -60-degree phase jump at 1/4 cycle 


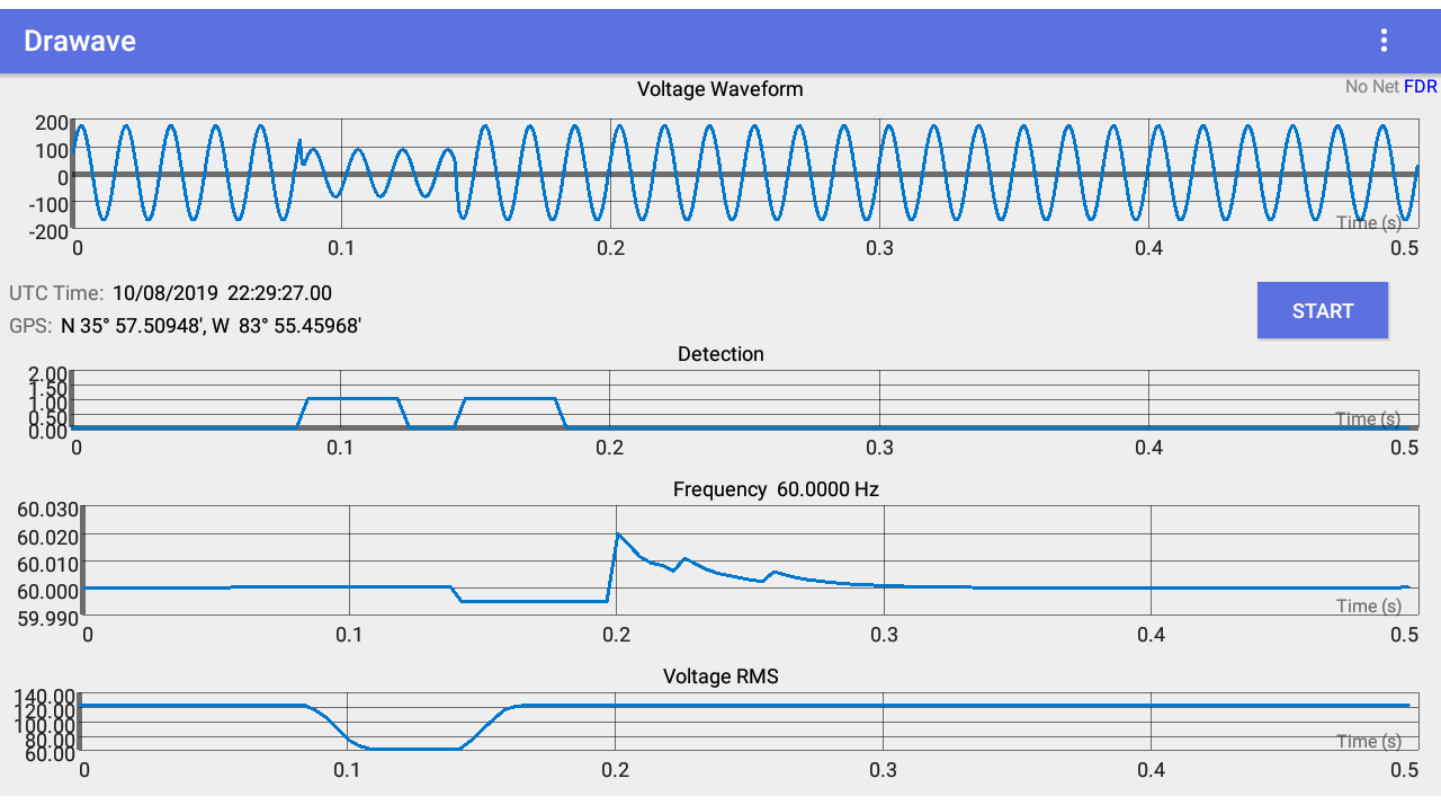

Figure 98 Real-time measurement result under -60-degree phase jump at 2/4 cycle

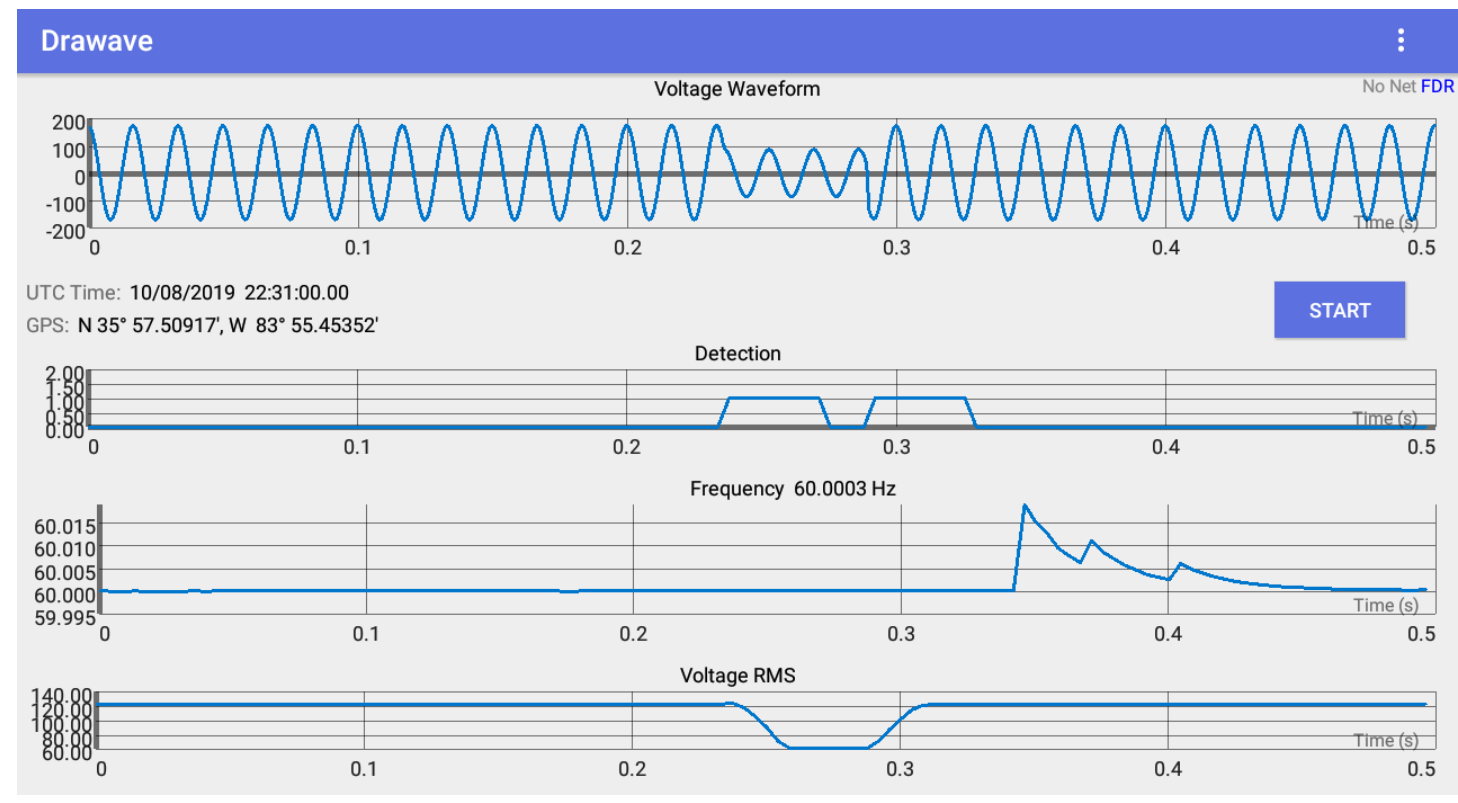

Figure 99 Real-time measurement result under -60-degree phase jump at 3/4 cycle

To summarize, the proposed fault-tolerant frequency measurement algorithm was implemented on lowcost hardware to realize real-time fault detection and frequency measurement. Experiments were conducted to test the real time performance. First, voltage sag with different magnitude from 10\% to $90 \%$ were used to test the frequency measurement algorithm. Then, voltage waveforms with phase angle jump were used for further tests. The experiment results verified that the proposed fault-tolerant frequency measurement algorithm has good performance in all test cases. Large frequency measurement errors incurred by conventional methods without fault-tolerant mechanism could be significantly reduced. And hence the reliability of frequency measurement was improved, and it could contribute to higher reliability of grid protection for solar power inverters. 


\section{Milestone \# 4}

The work in section 5.2 and section 5.3 completed milestone \# 4.

\begin{tabular}{|l|l|l|}
\hline Milestone & Metric Definition & $\begin{array}{l}\text { Finished } \\
\text { Date }\end{array}$ \\
\hline 4 & $\begin{array}{l}\text { Practicality of the algorithm demonstrated by analyzing the } \\
\text { computational complexity of the developed algorithm in terms of } \\
\text { multiplications and additions, and space requirement, comparing } \\
\text { with modern low-cost processor, and demonstration on low-cost } \\
\text { FNET/GridEye sensors }\end{array}$ & $09 / 30 / 2019$ \\
\hline
\end{tabular}

\subsection{GE INVERTER TESTING IN RTDS}

\subsubsection{Test Case Playback in RTDS}

The test case \# 1 to 12 performed in section 5.1.4 dependability test has been performed in the RTDS with GE Brilliance $1 \mathrm{MW}$ inverter hardware in loop. The system setup is shown in Figure 100 and the RTDS data playback model is shown in Figure 101. The inverter's response is shown in Figure 102 - Figure 113. In each test case, the terminal's RMS voltage, inverter's current, frequency measured by PLL, and inverter output power are shown. It should be noted that the PLL frequency is measured in the fastest task in the inverter control. It is used for the inverter control including the transient control during the fault ride through and is not used for protection. In GE inverter, a comprehensive and robust protection algorithm is designed for the frequency related protection.

The transient fault with phase angle step change was applied around 0.25 second and lasts for about 3 fundamental frequency cycles. We can see the voltage distortion in first plot and corresponding frequency measurement distortions in the third plot. In these tests, the GE inverter successful rode through without erroneous trips. The reason for this is as mentioned earlier: GE inverter does not do the protection based on the fast PLL frequency measurements but instead uses dedicated logic to provide those protections. Although we can see the large frequency measurement swings during the system transient events, they don't trip the inverter during the transient. On the contrary, this measurement is necessary for the quick identification of the grid transient events and therefore provides corrective control measures to ride through the transients.

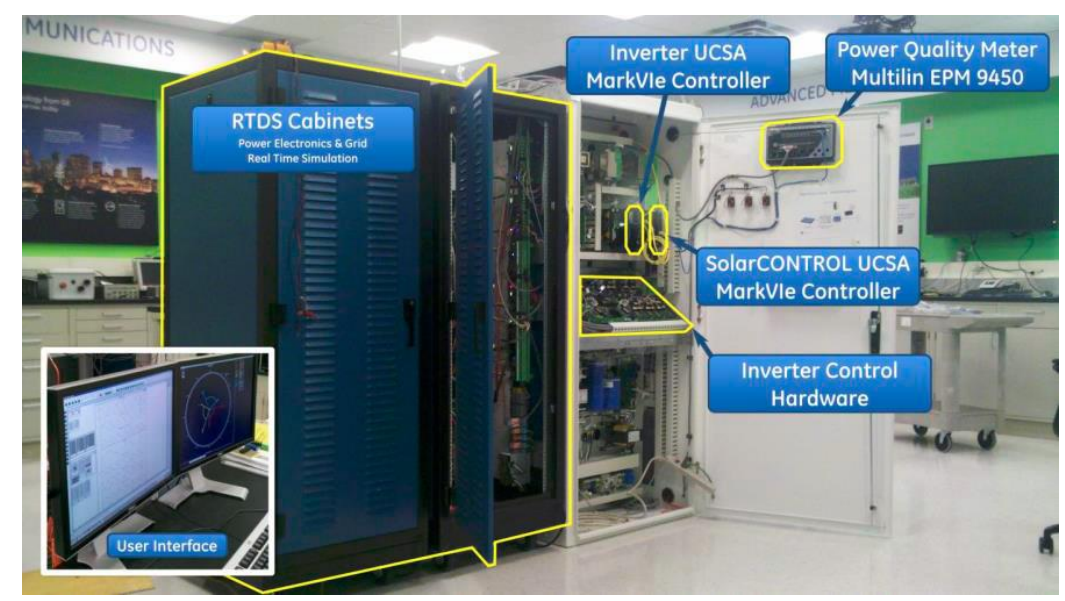

Figure 100 GE Brilliance inverter HIL system 


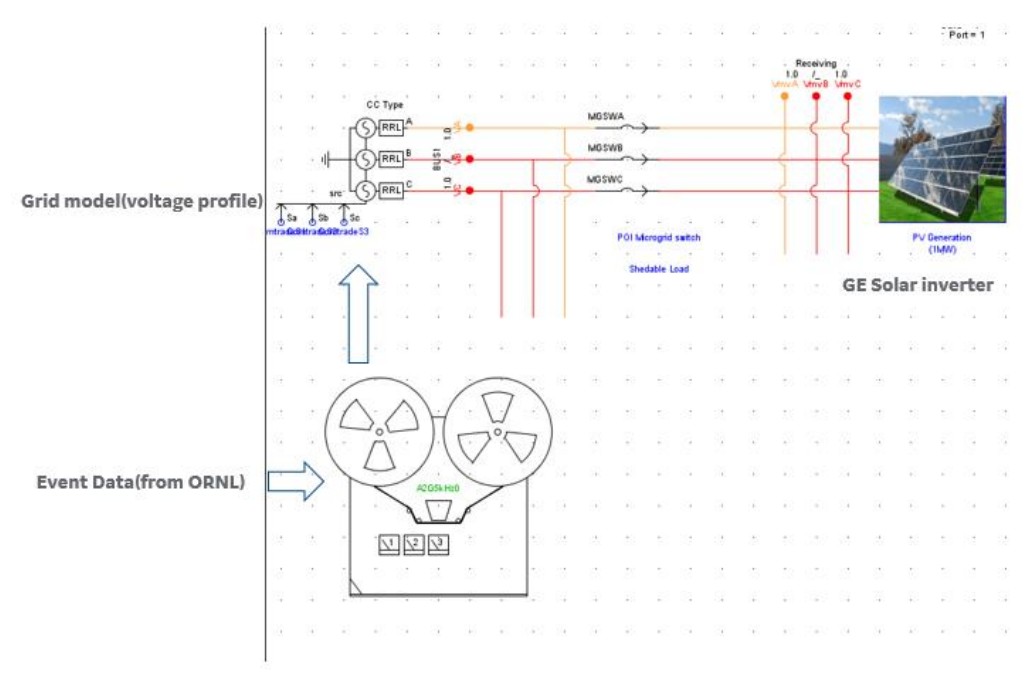

Figure 101 The RTDS model used for the test
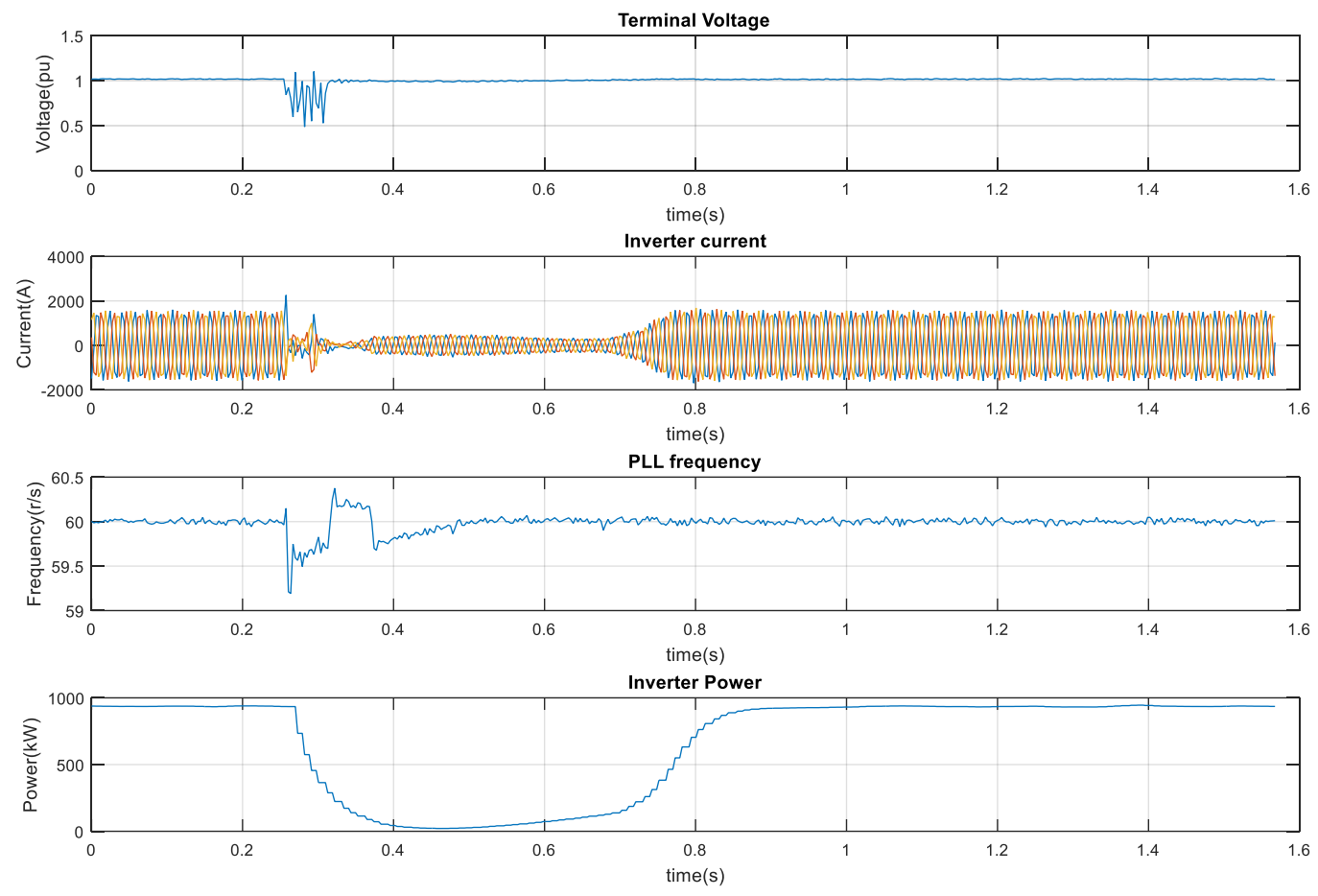

Figure 102 GE Brilliance inverter response (test case 1) 

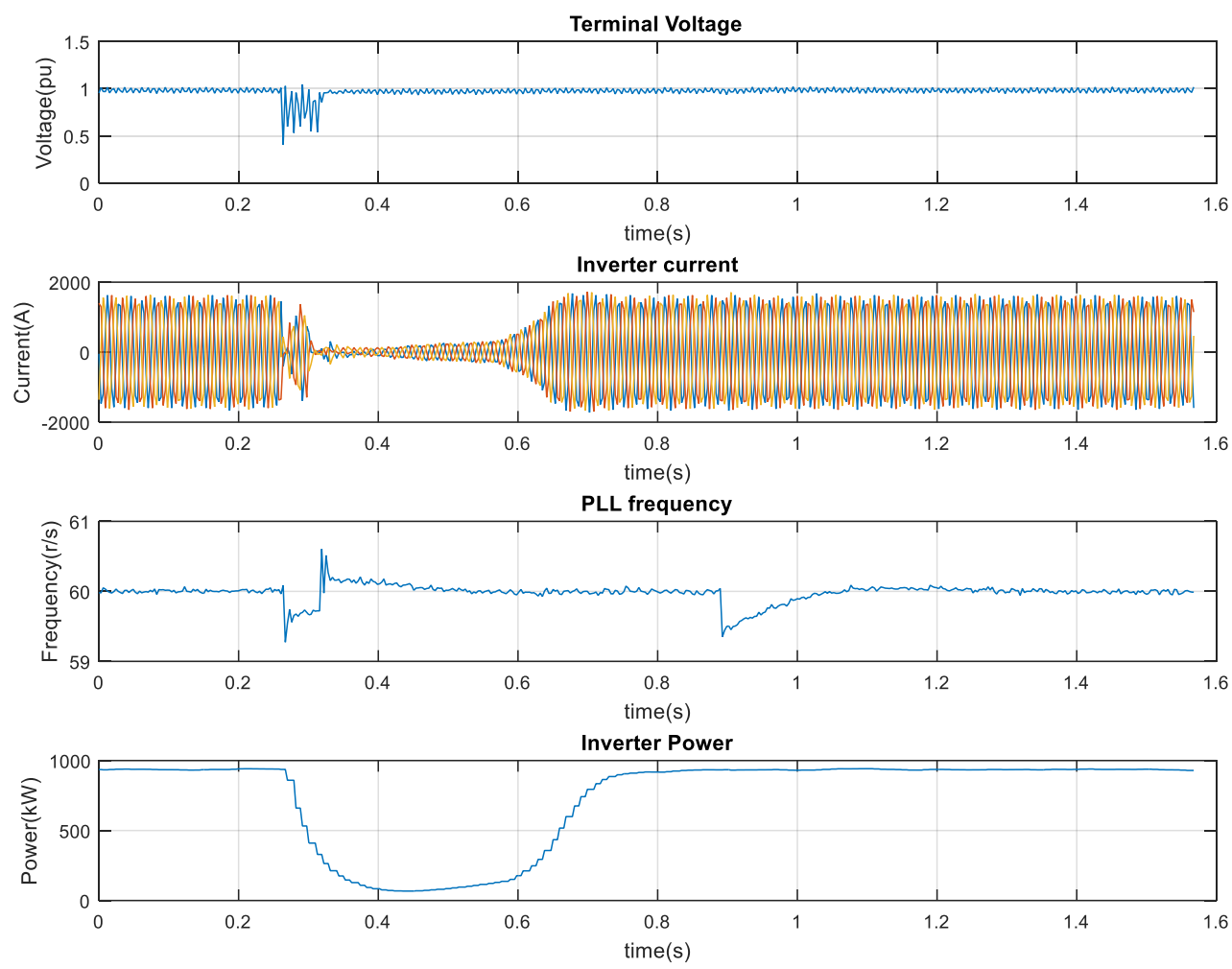

Figure 103 GE Brilliance inverter response (test case 2)
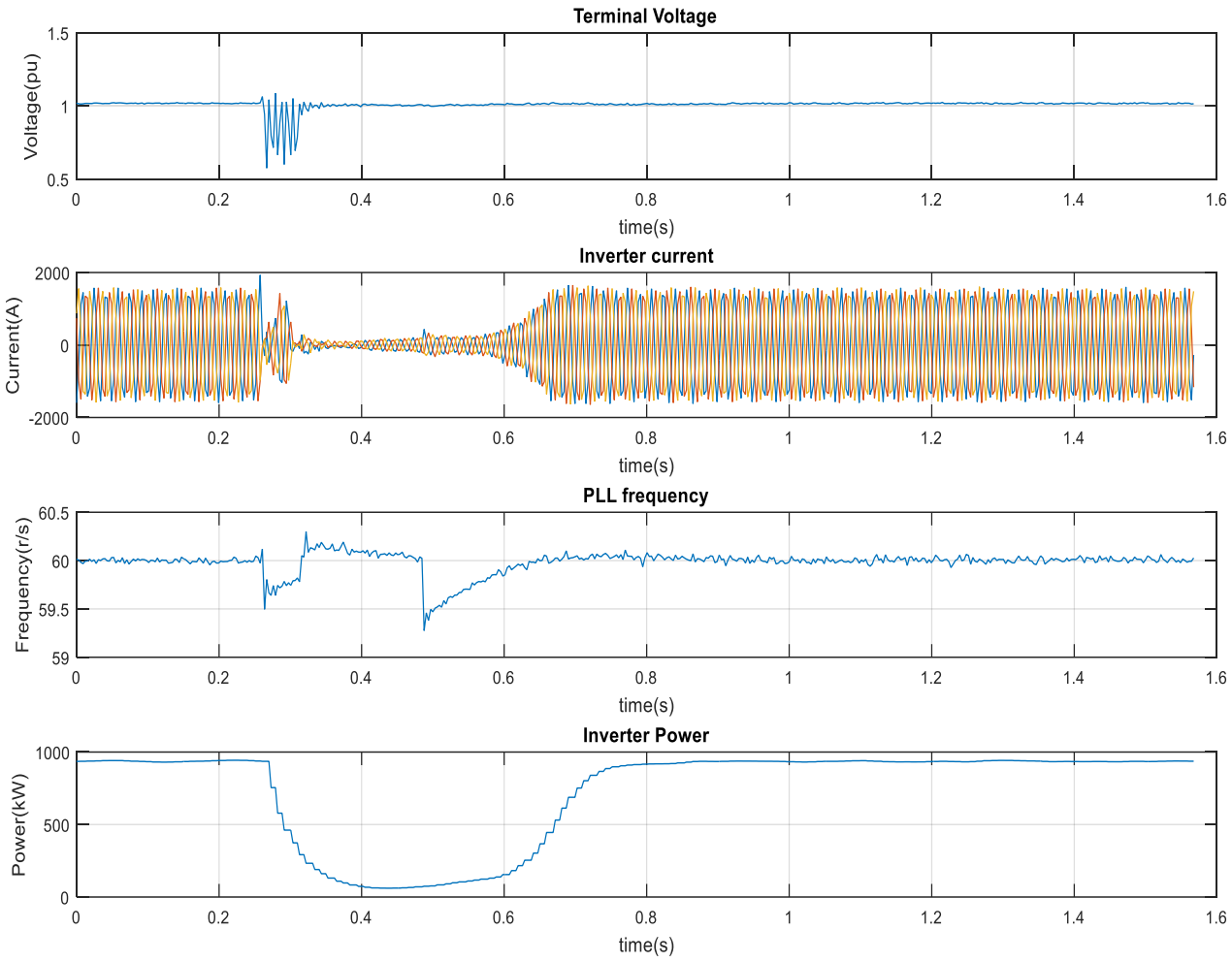

Figure 104 GE Brilliance inverter response (test case 3) 


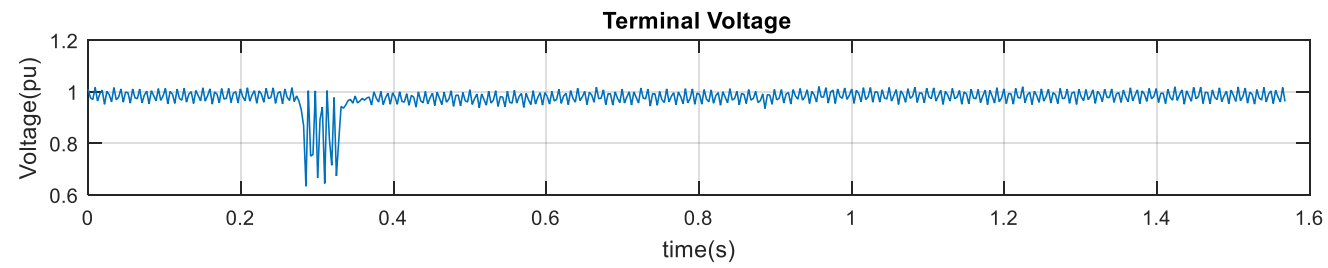

Inverter current

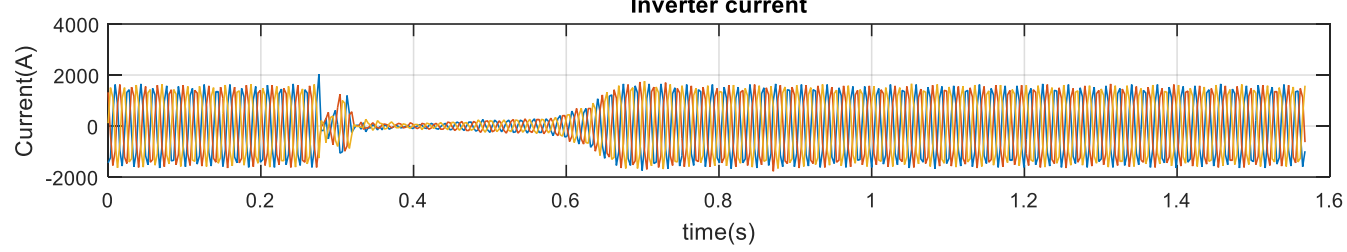

PLL frequency

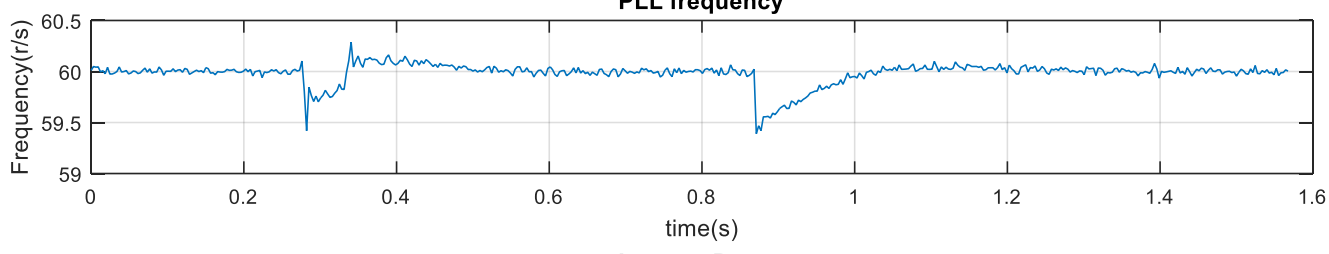

Inverter Power

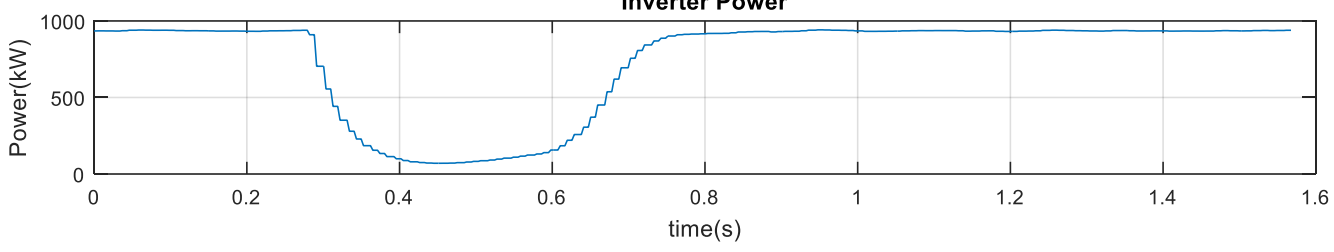

Figure 105 GE Brilliance inverter response (test case 4)
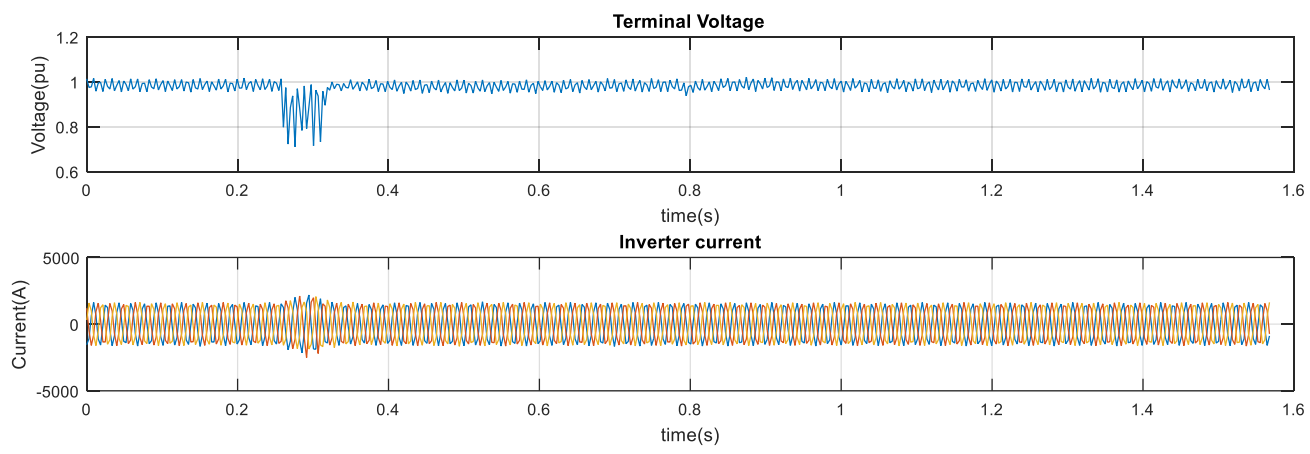

PLL frequency
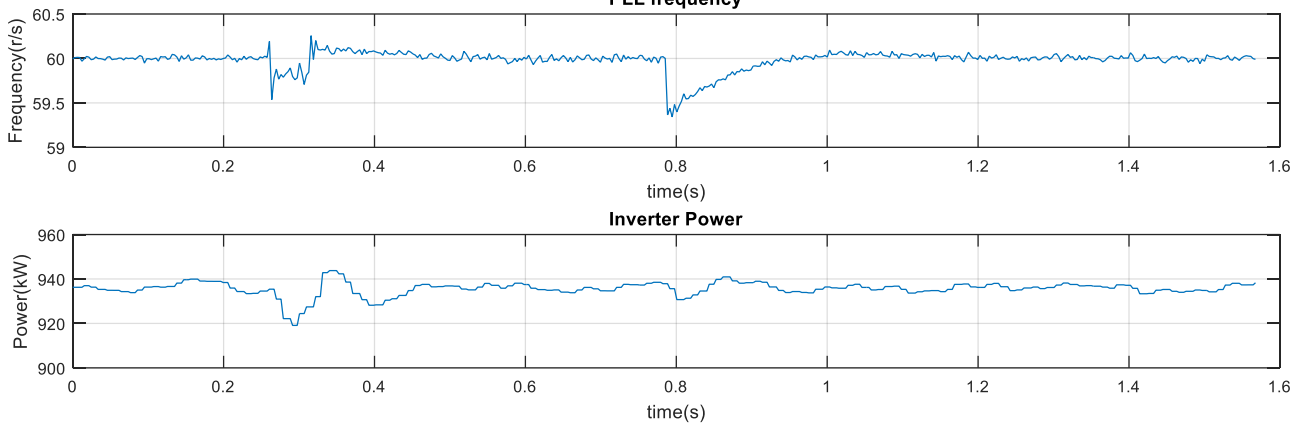

Figure 106 GE Brilliance inverter response (test case 5) 

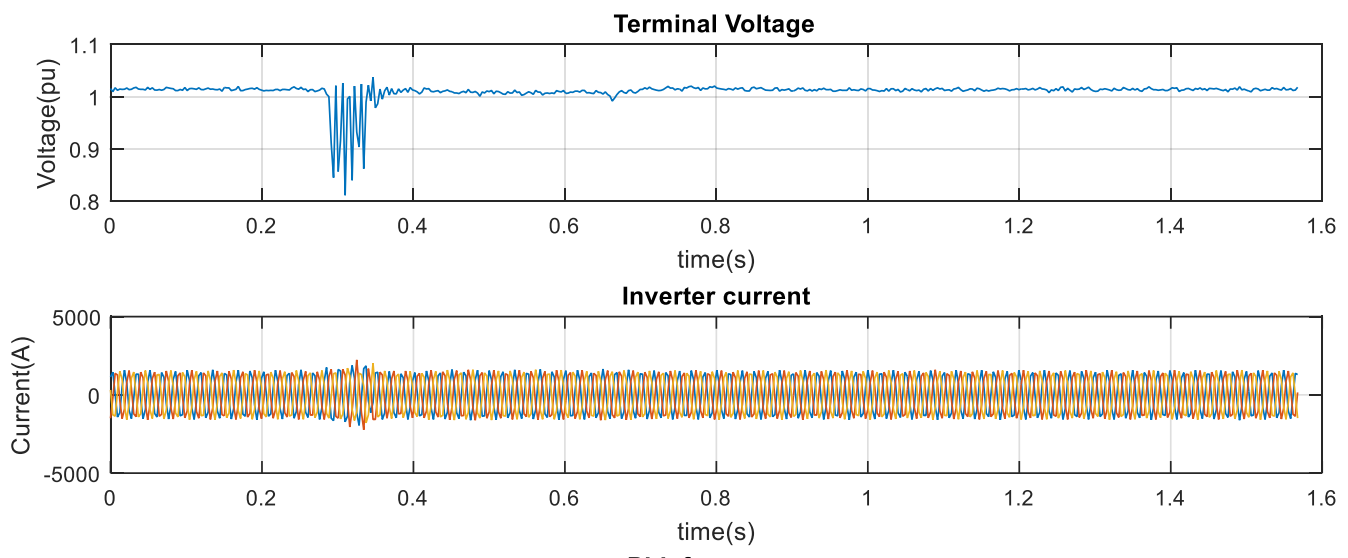

PLL frequency
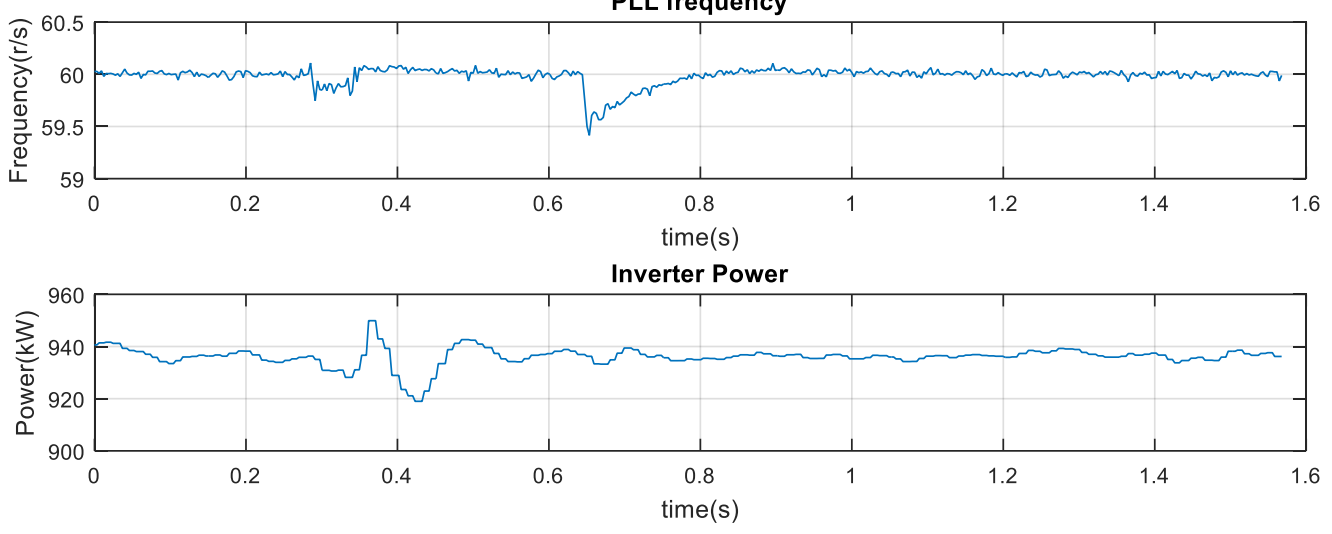

Figure 107 GE Brilliance inverter response (test case 6)
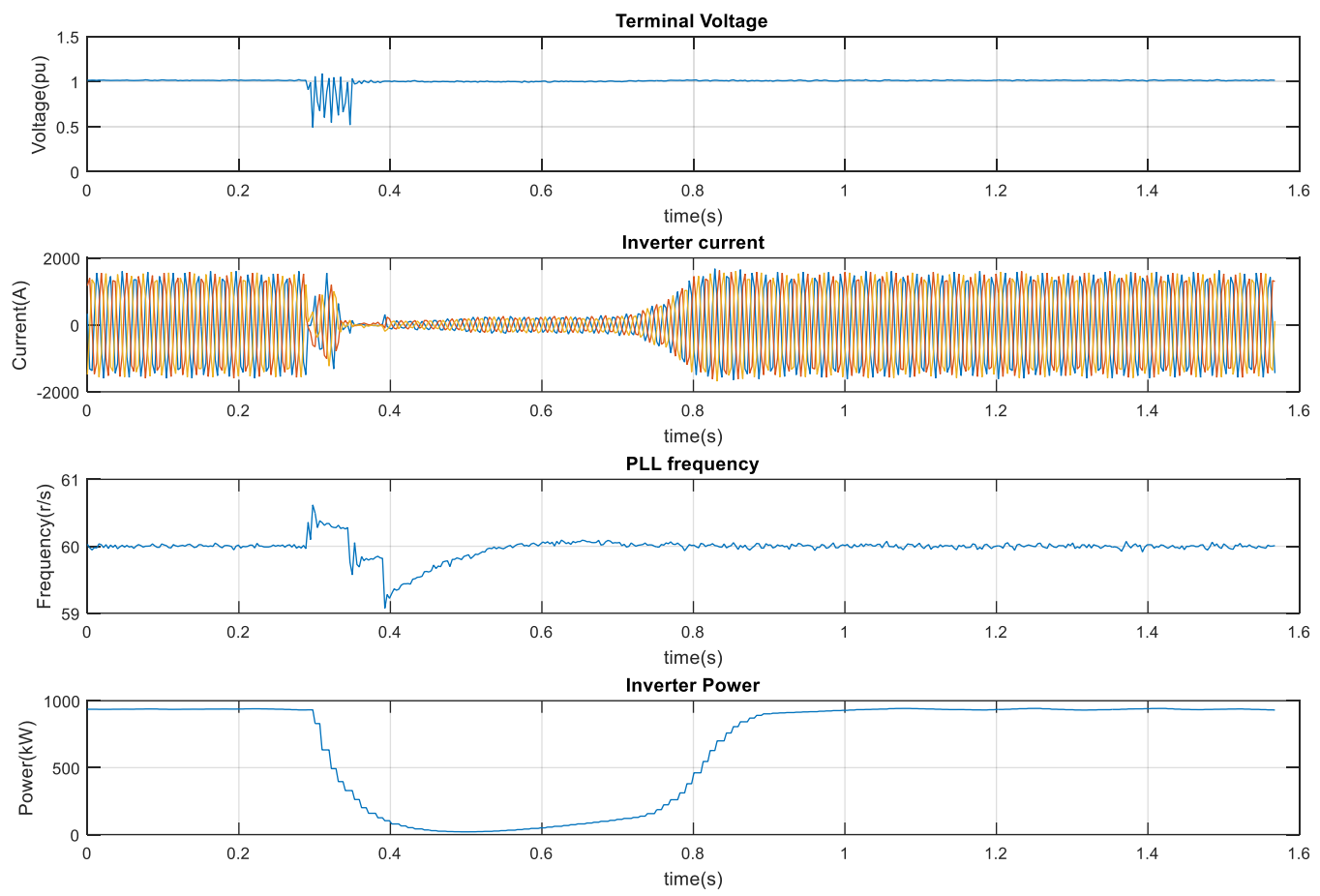

Figure 108 GE Brilliance inverter response (test case 7) 

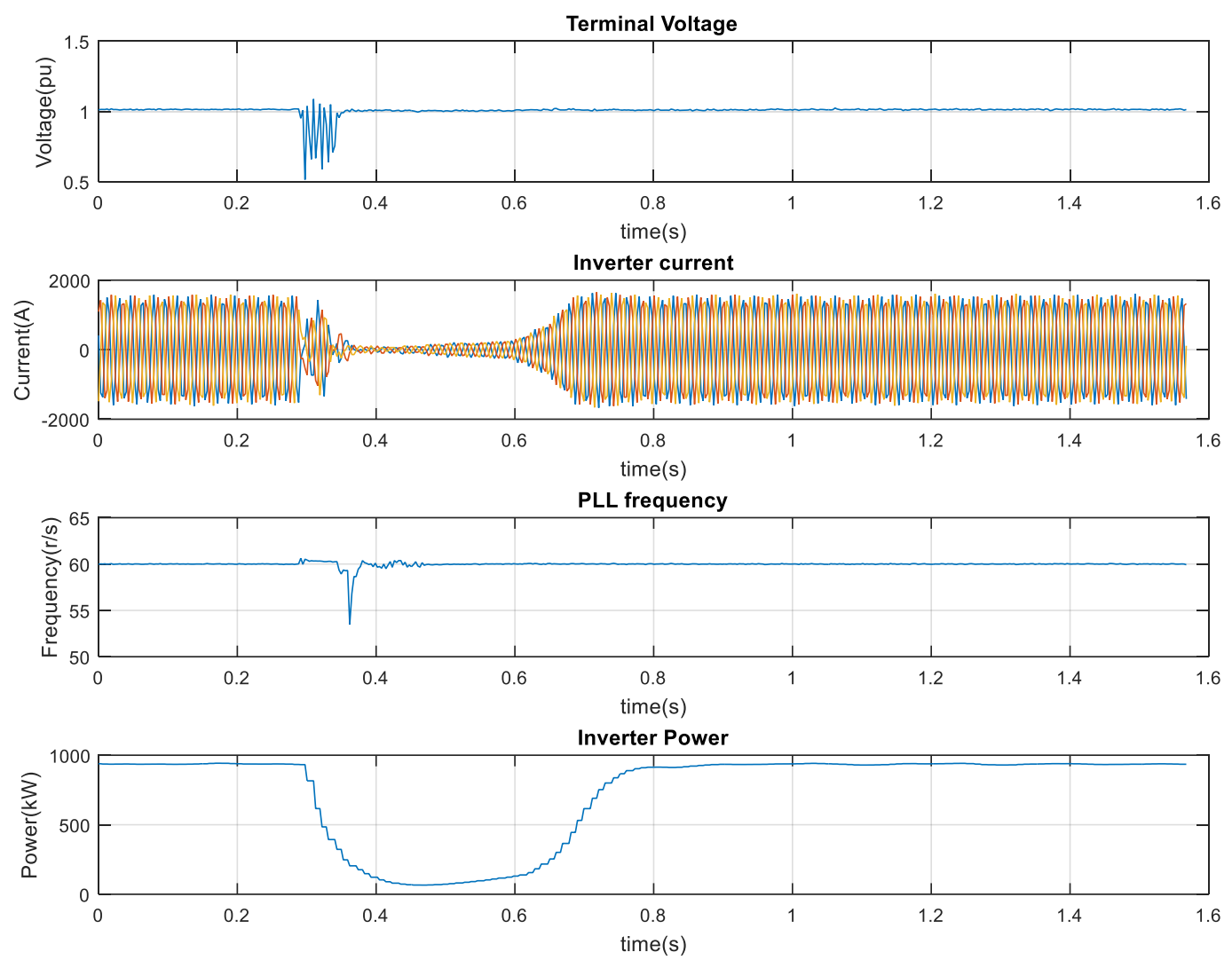

Figure 109 GE Brilliance inverter response (test case 8)
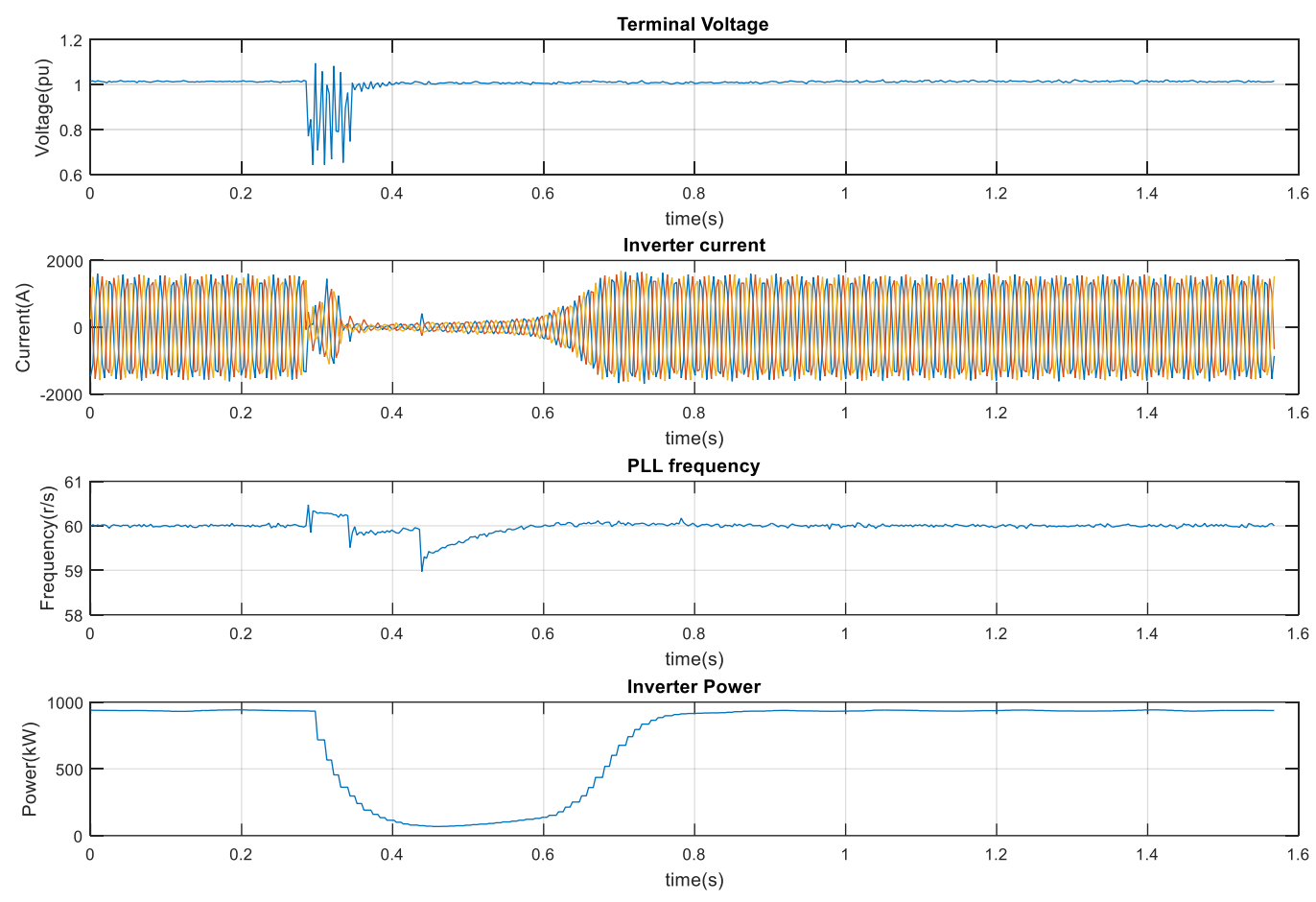

Figure 110 GE Brilliance inverter response (test case 9) 

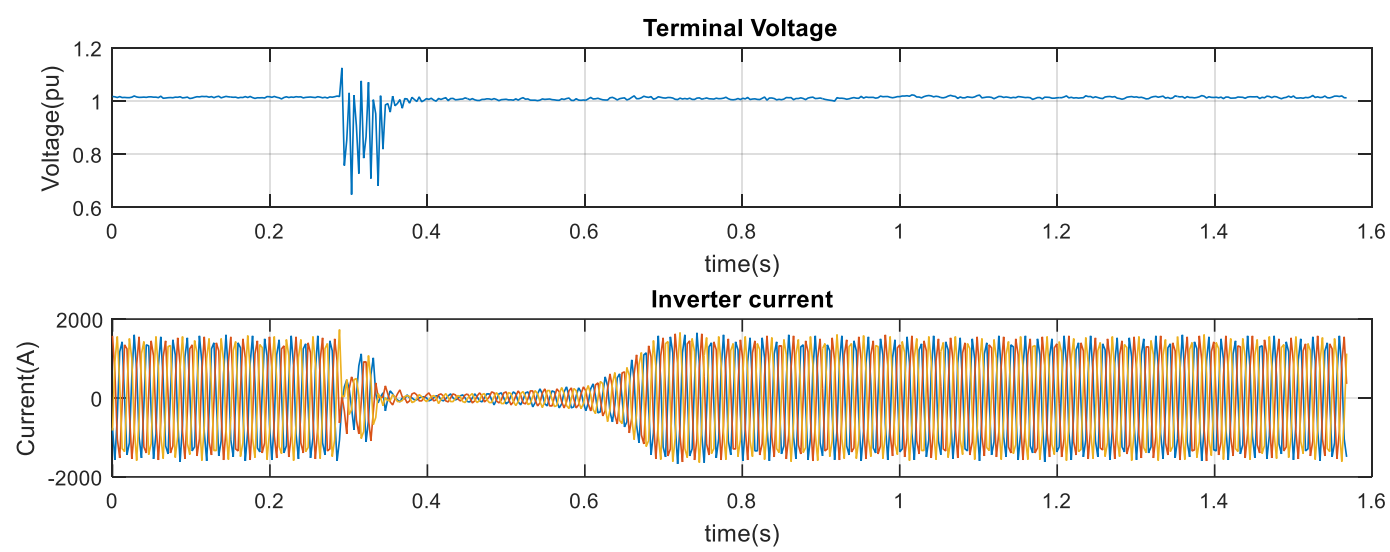

PLL frequency

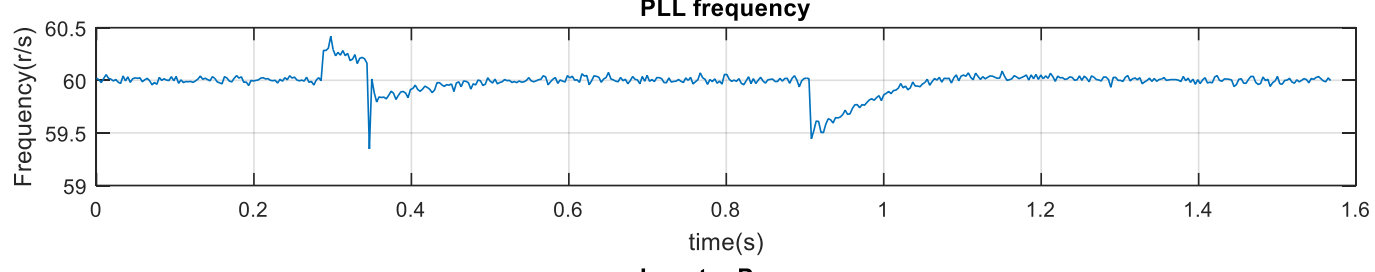

Inverter Power

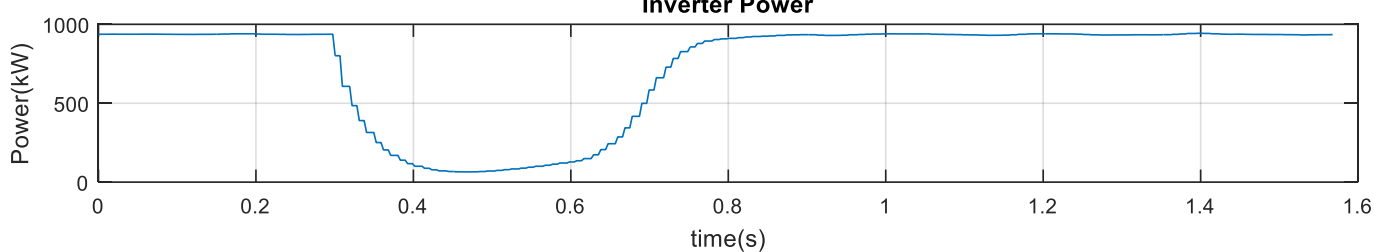

Figure 111 GE Brilliance inverter response (test case 10)
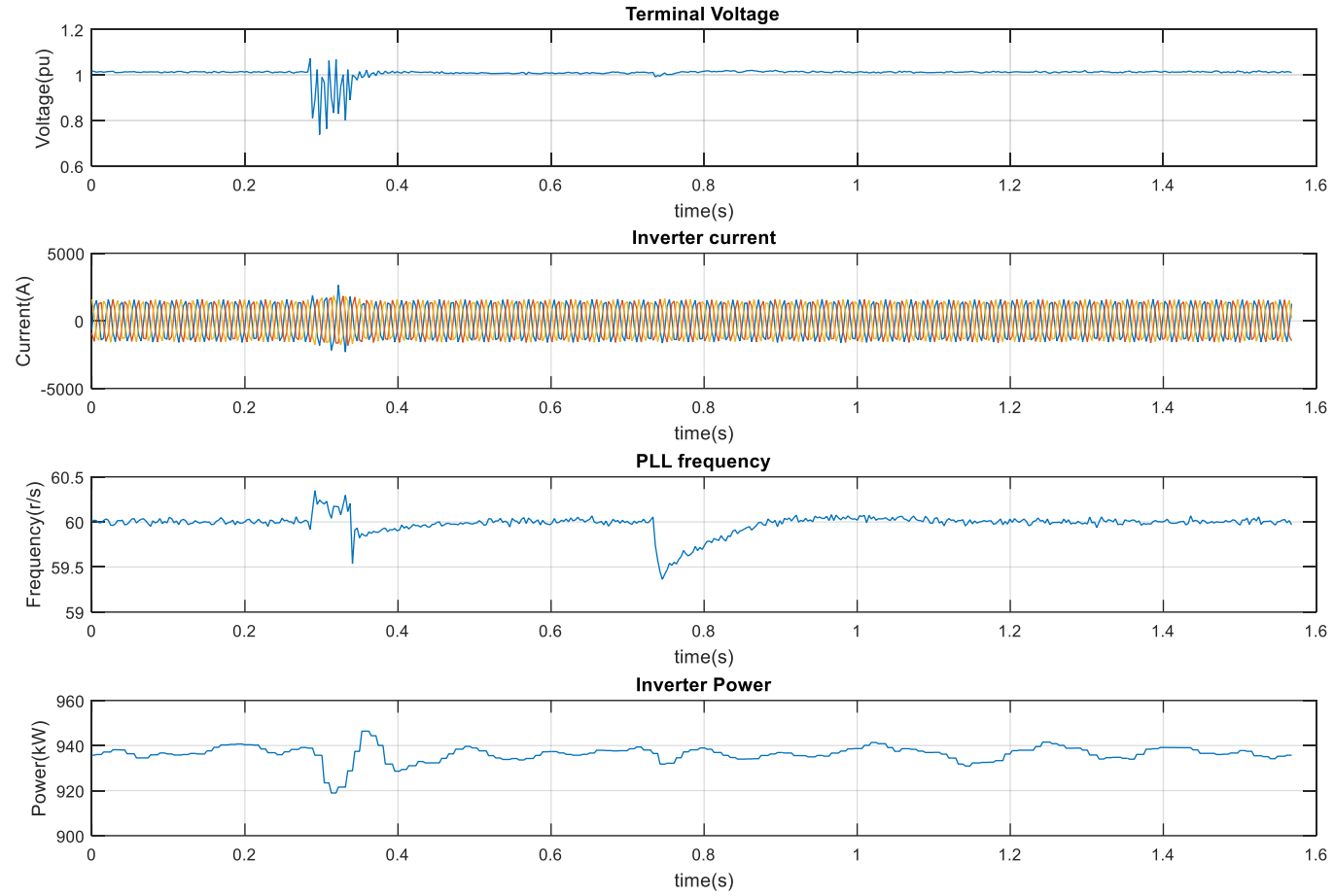

Figure 112 GE Brilliance inverter response (test case 11) 

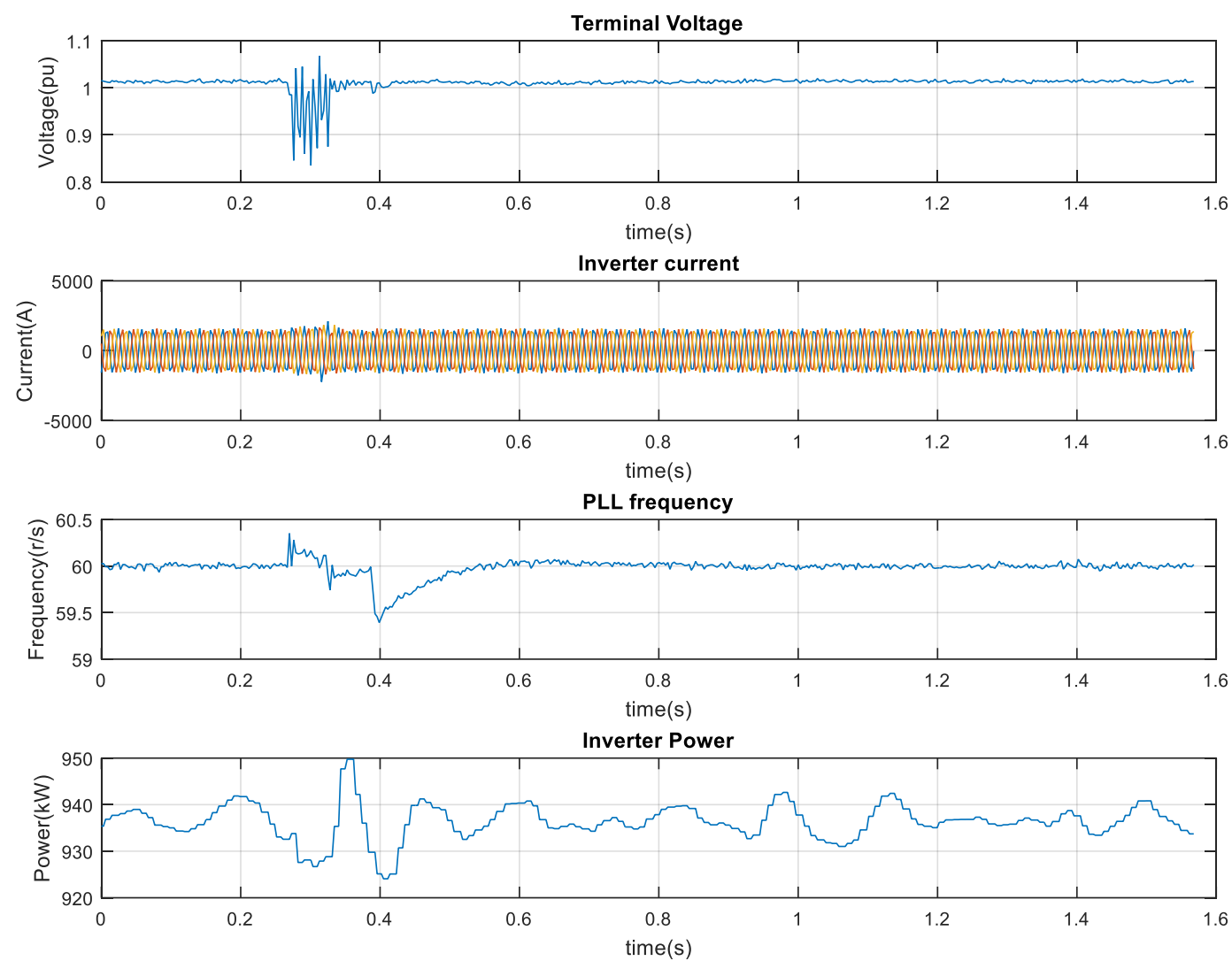

Figure 113 GE Brilliance inverter response (test case 12)

\subsubsection{IEEE 13 node system test}

To further test the response of GE 1 MW commercial solar inverter to grid events, the IEEE 13 Node test feeder is integrated into the RTDS with $1 \mathrm{MW}$ brilliance solar inverter. The IEEE 13 node system is operated at $4.16 \mathrm{kV}$ and is widely used to test common features of distribution analysis software. It is characterized by being short, relatively highly loaded, a single voltage regulator at the substation, overhead and underground lines, shunt capacitors, an in-line transformer, and unbalanced loading. An illustration of the system is shown in Figure 114 and Table XVIII highlights the overall characteristics of this system. Also, for the purpose of this study, an additional three-phase capacitor bank is added at Node 680. More details of the system are listed in Appendix B. 


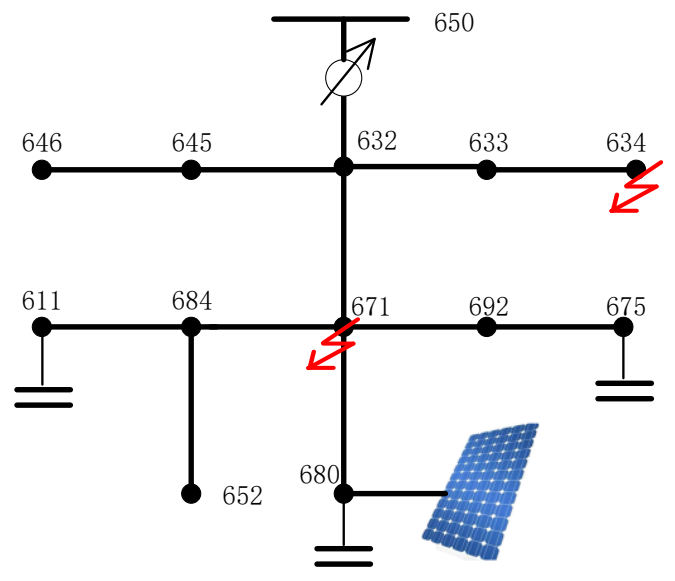

Figure 114 IEEE 13 system model used for the test and the PV connection

Table XVIII IEEE 13 Node System overview

\begin{tabular}{|l|l|}
\hline & IEEE 13 \\
\hline Volt Level & $4.16 \mathrm{kV}$ \\
\hline No. of Nodes & 13 \\
\hline No. of Line Sections & 12 \\
\hline Total Conductor Length & $8200 \mathrm{ft}(2.5 \mathrm{~km})$ \\
\hline Total Load & $3.47 \mathrm{MW}$ \\
\hline No. of Regulators & 1 \\
\hline No. of Capacitor Banks & $1(3-\mathrm{Ph})+1(1-\mathrm{Ph})$ \\
\hline
\end{tabular}

As shown in Figure 114, the GE 1 MW solar inverter was integrated into the grid through Node 680. A model overview in RTDS is shown in Figure 115.

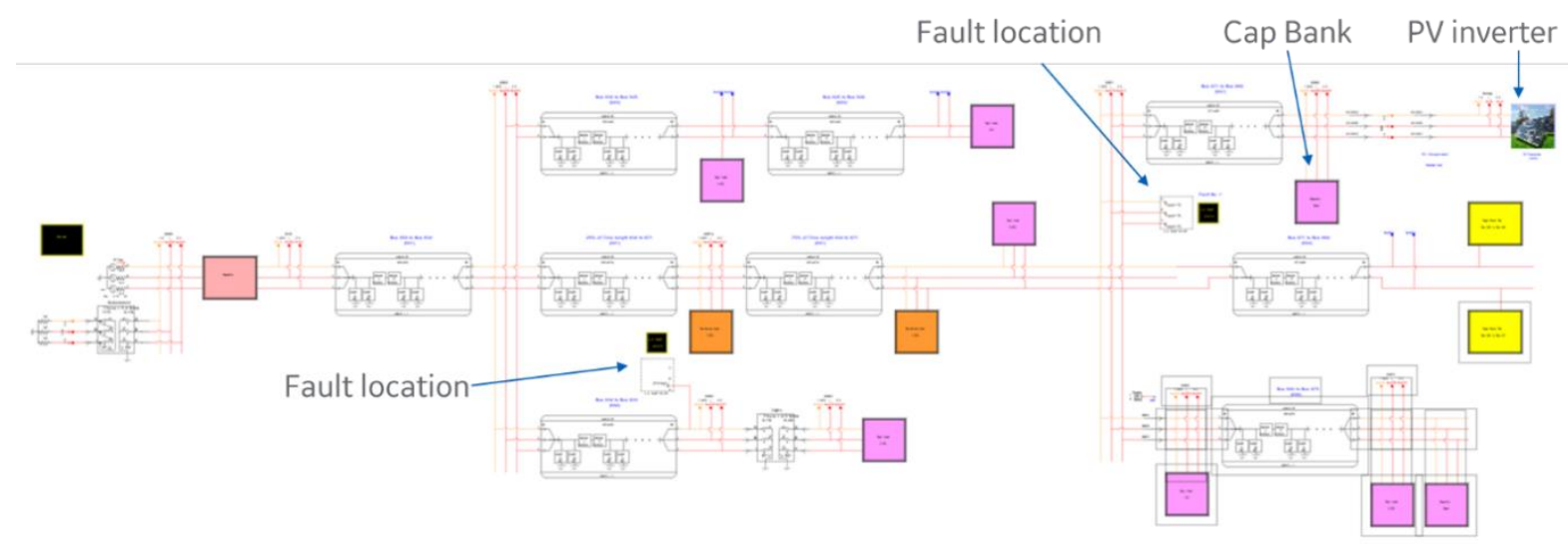

Figure 115 Test system in RTDS

\subsubsection{Test scenarios}

To test the performance of GE inverter, especially the frequency related protection, the following test scenarios are defined: 
- 2 fault locations: Node 671 and Node 634

- 3 fault types: single line to ground (SLG), line to line to ground (LLG), and three-phase fault (3ph)

- 2 fault durations: 5 cycles and 60 cycles

- 2 capacitor switching events: single phase capacitor and three-phase capacitor

The focus of the tests is the frequency measurements inside the inverter and how the inverter is responding to the events. For comparison, both the PLL frequency and grid monitor frequency were captured during the tests. PLL frequency is used by the control and grid monitor frequency is used for the protection purpose or other grid compliance related algorithms.

For better reference of the test results in the following section, the following cases are defined.

- Case 1: A LLG fault event at Node 671 with fault duration of 5 cycles

- Case 2: A LLG fault event at Node 671 with fault duration of 60 cycles

- Case 3: A 3-Ph fault event at Node 671 with fault duration of 5 cycles

- Case 4: A 3-Ph fault event at Node 671 with fault duration of 60 cycles

- Case 5: A SLG fault event at Node 634 with fault duration of 5 cycles

- Case 6: A SLG fault event at Node 634 with fault duration of 60 cycles

- $\quad$ Case 7: 1-Ph capacitor switch on at Node 680

- $\quad$ Case 8: 3-Ph capacitor switch on at Node 680

\subsubsection{Test results}

Both the inverter internal recordings and the RTDS runtime recordings were captured in the tests. The inverter side waveforms were captured using high speed capture buffer (depending on the total duration of data capture, different sampling rate might be used) to provide a better view of inverter internal variables during the transient. The RTDS runtime recordings are only for the accessible measurement points. The following signals are captured at the inverter side: inverter terminal voltage magnitude, PLL frequency, grid monitor frequency and inverter power; at the RTDS side, the grid voltages and currents are captured. 

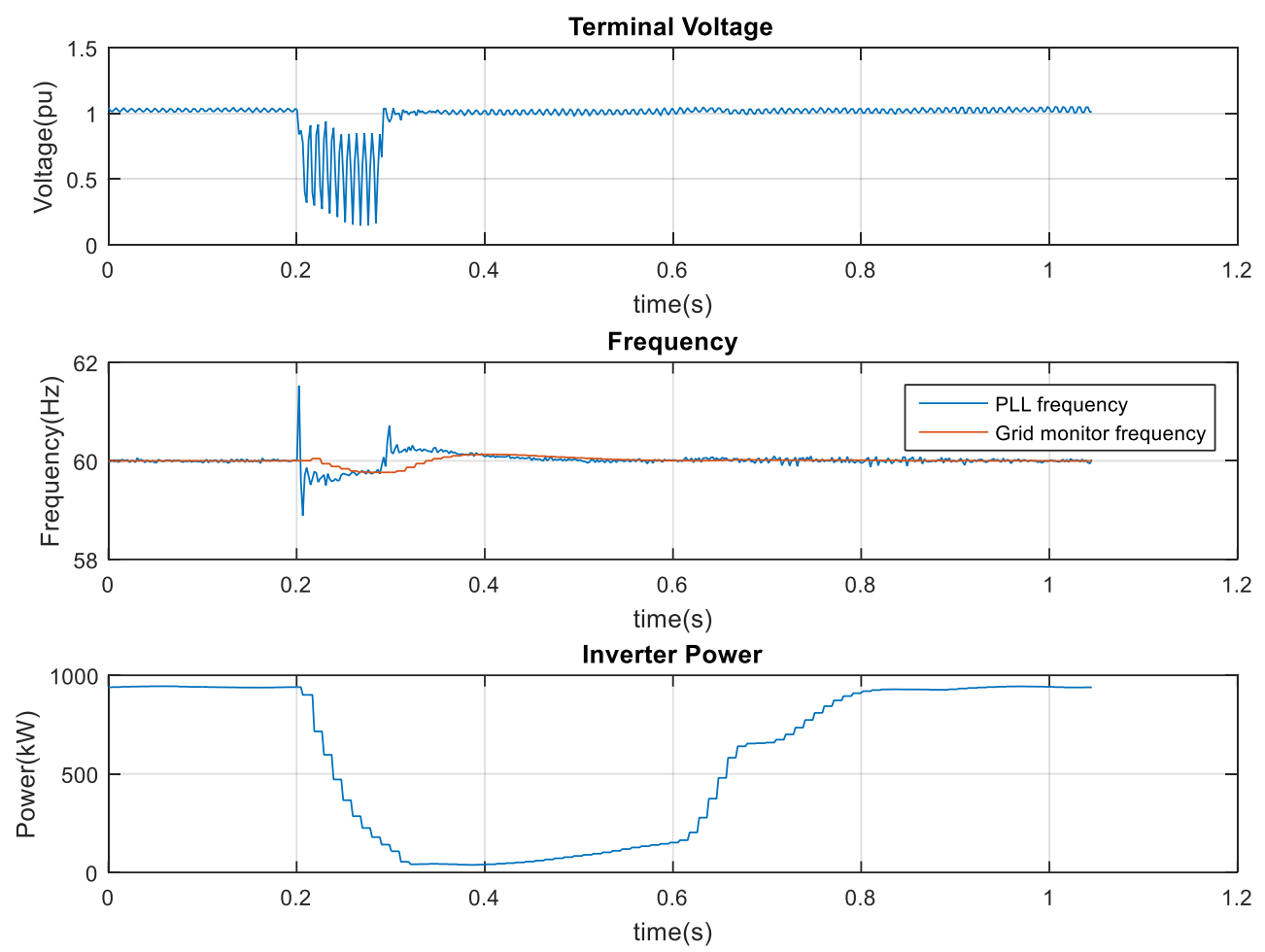

Figure 116 Inverter measurements for case1
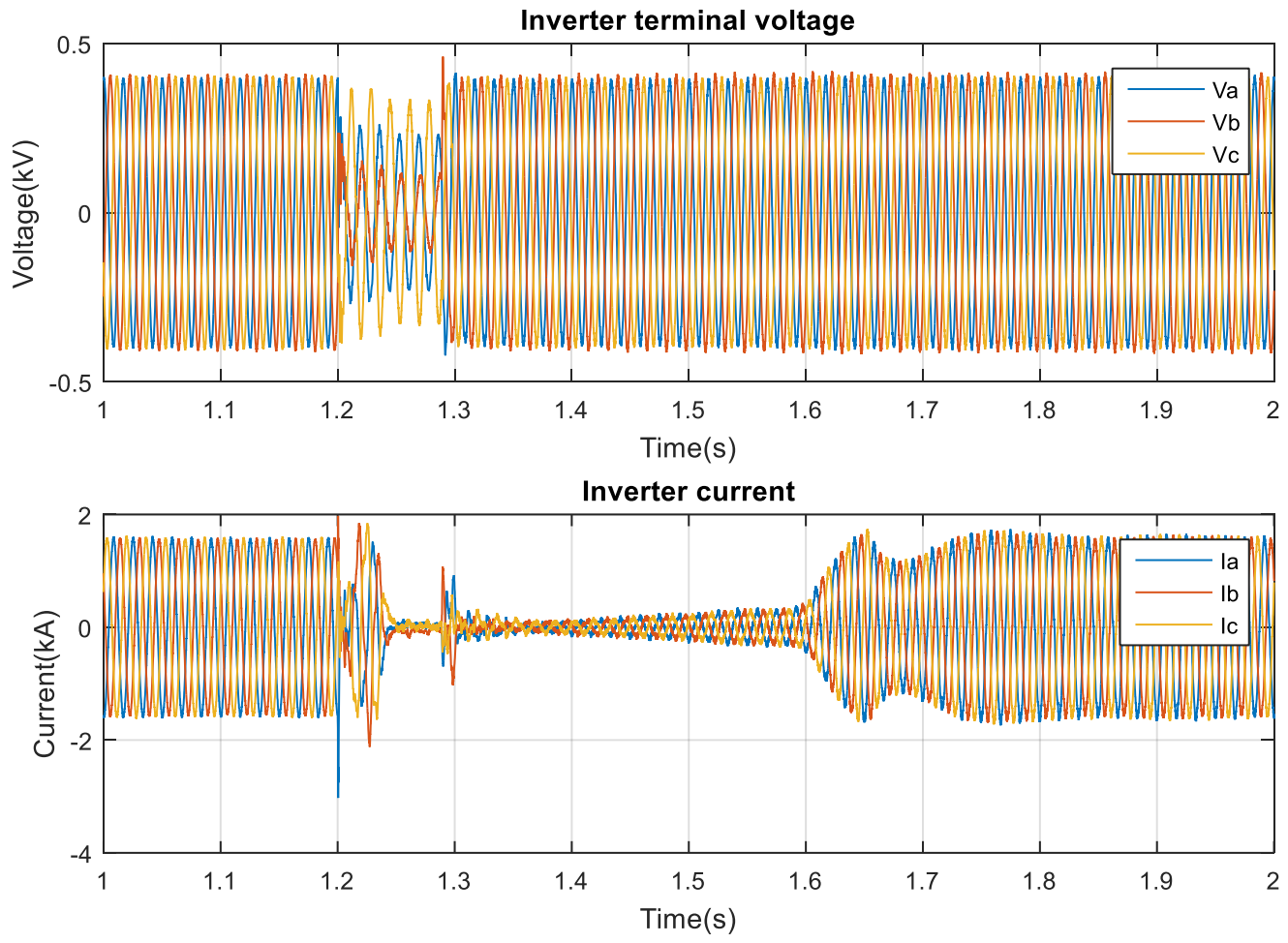

Figure 117 Voltage and currents for case1 


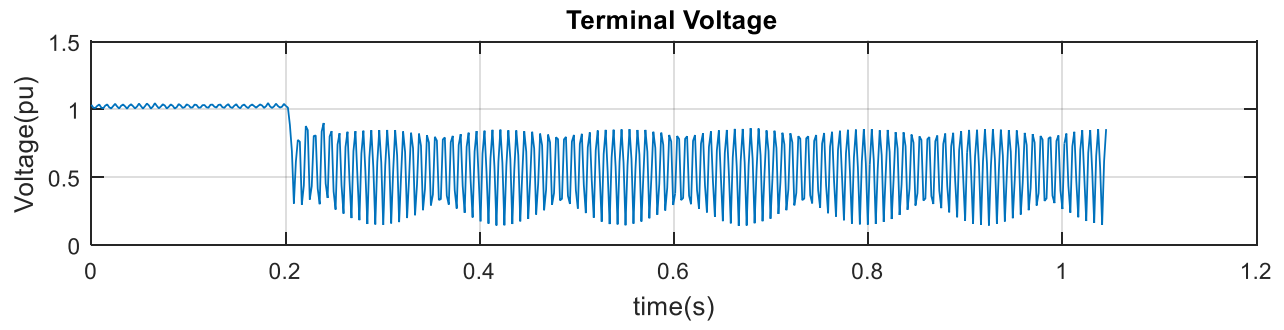

Frequency

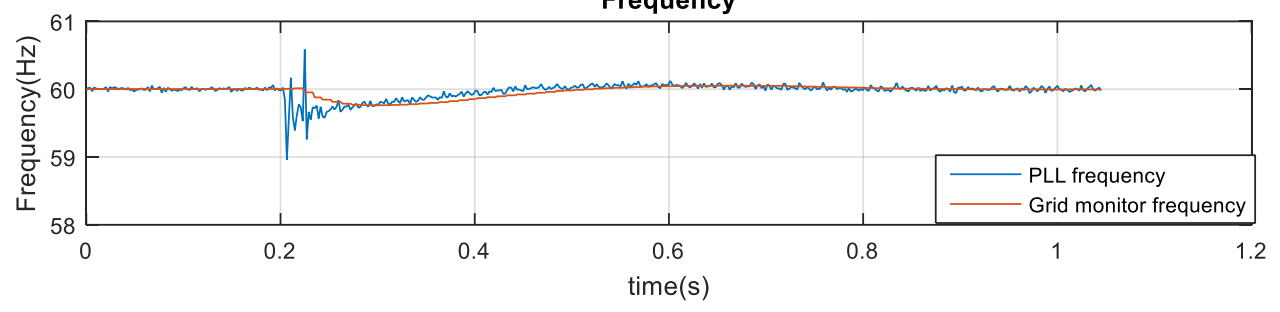

Inverter Power

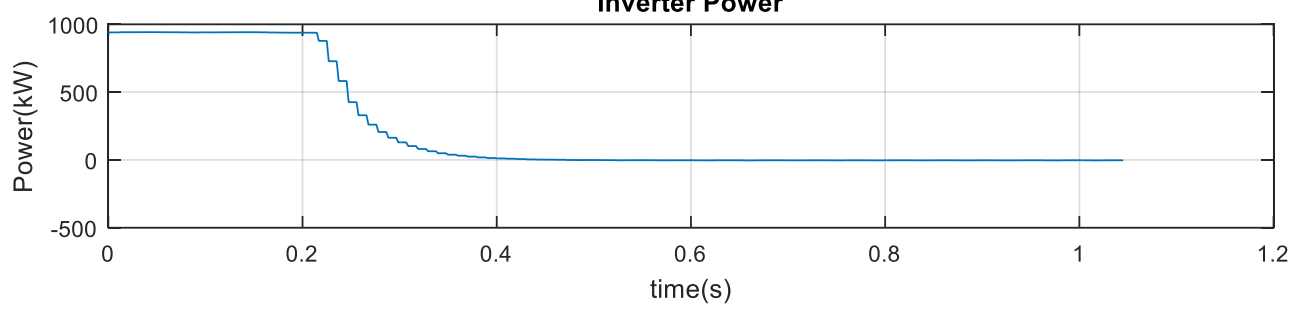

Figure 118 Inverter measurements for case2
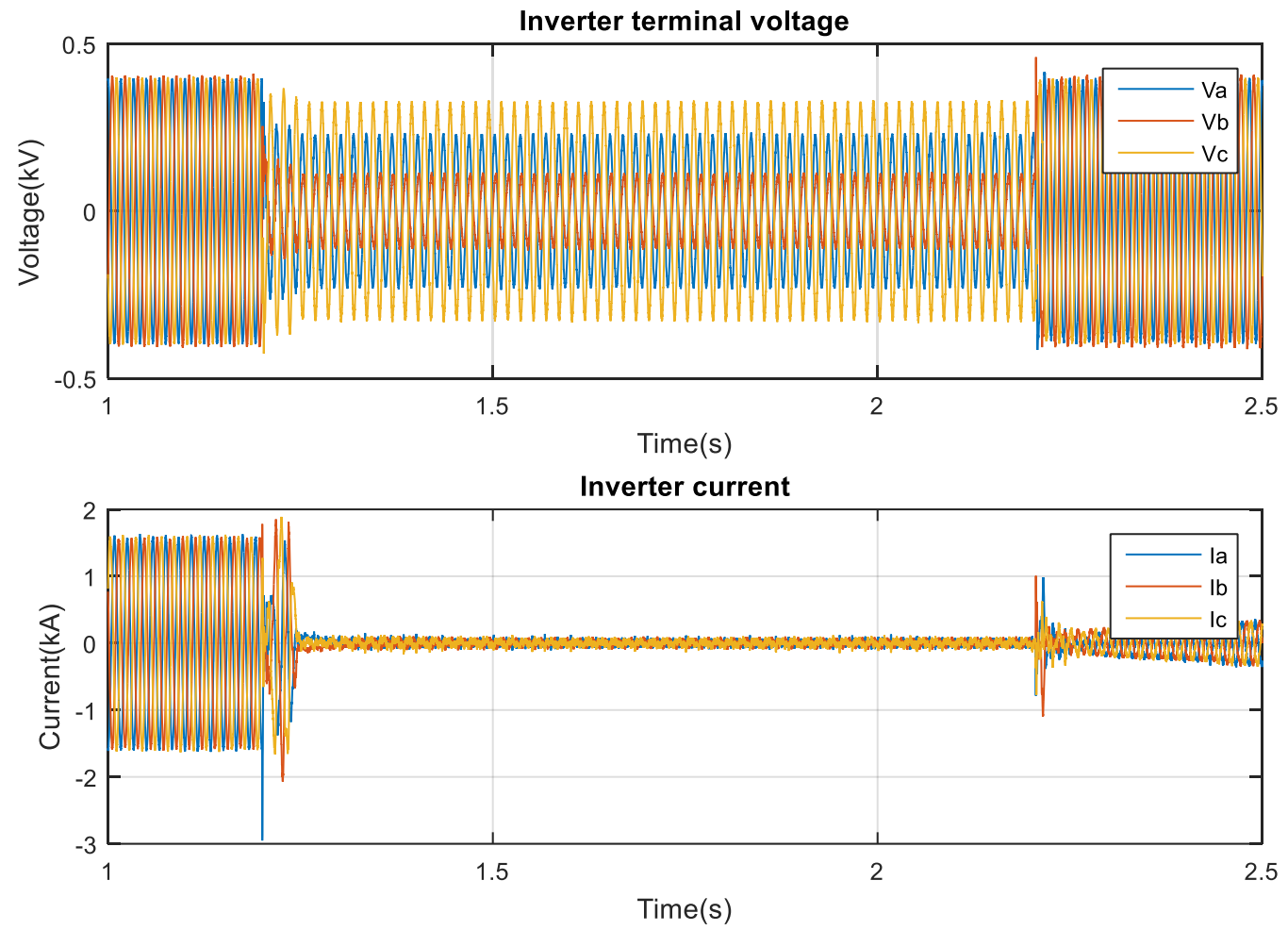

Figure 119 Voltage and currents for case2 

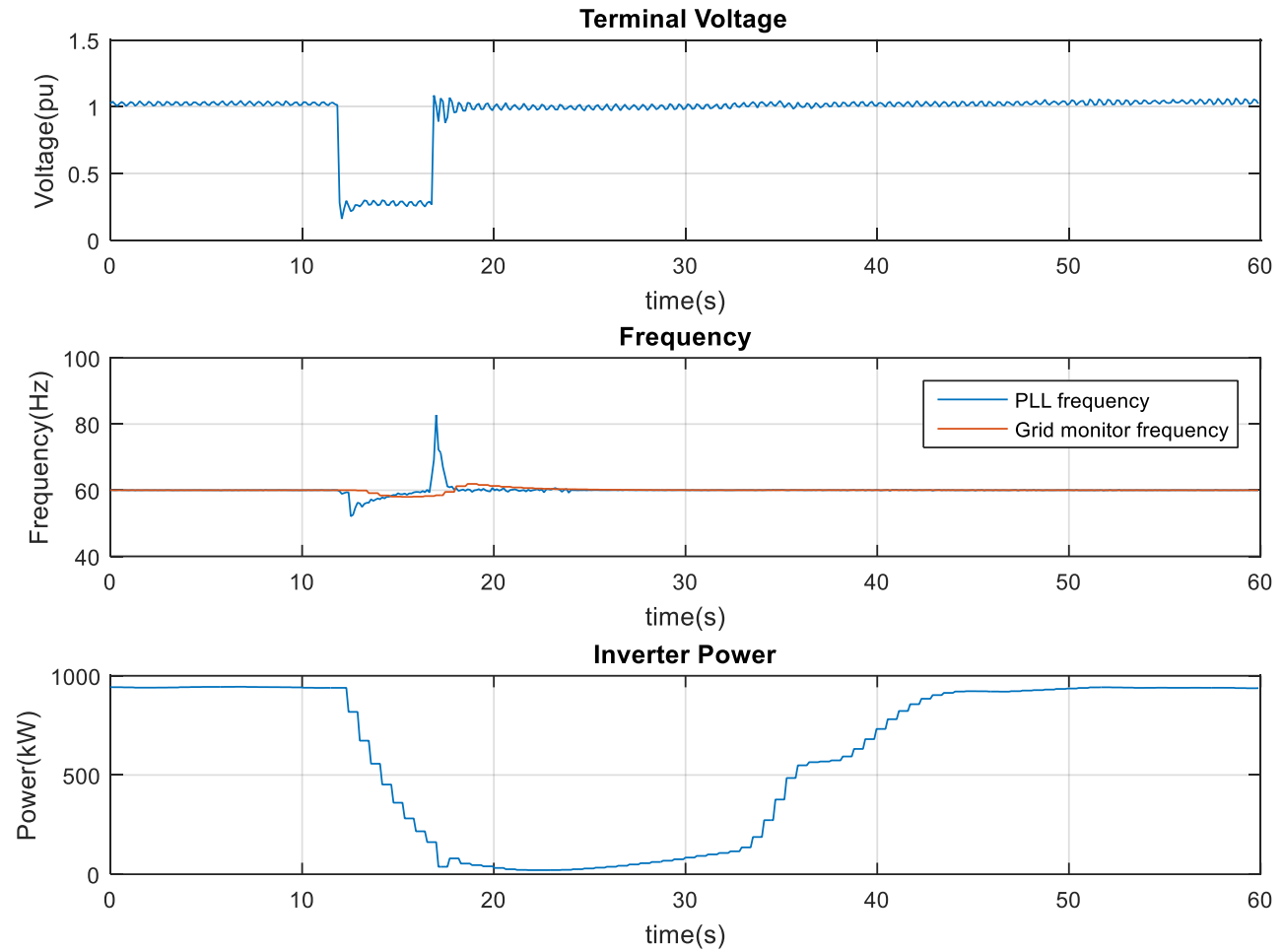

Figure 120 Inverter measurements for case 3
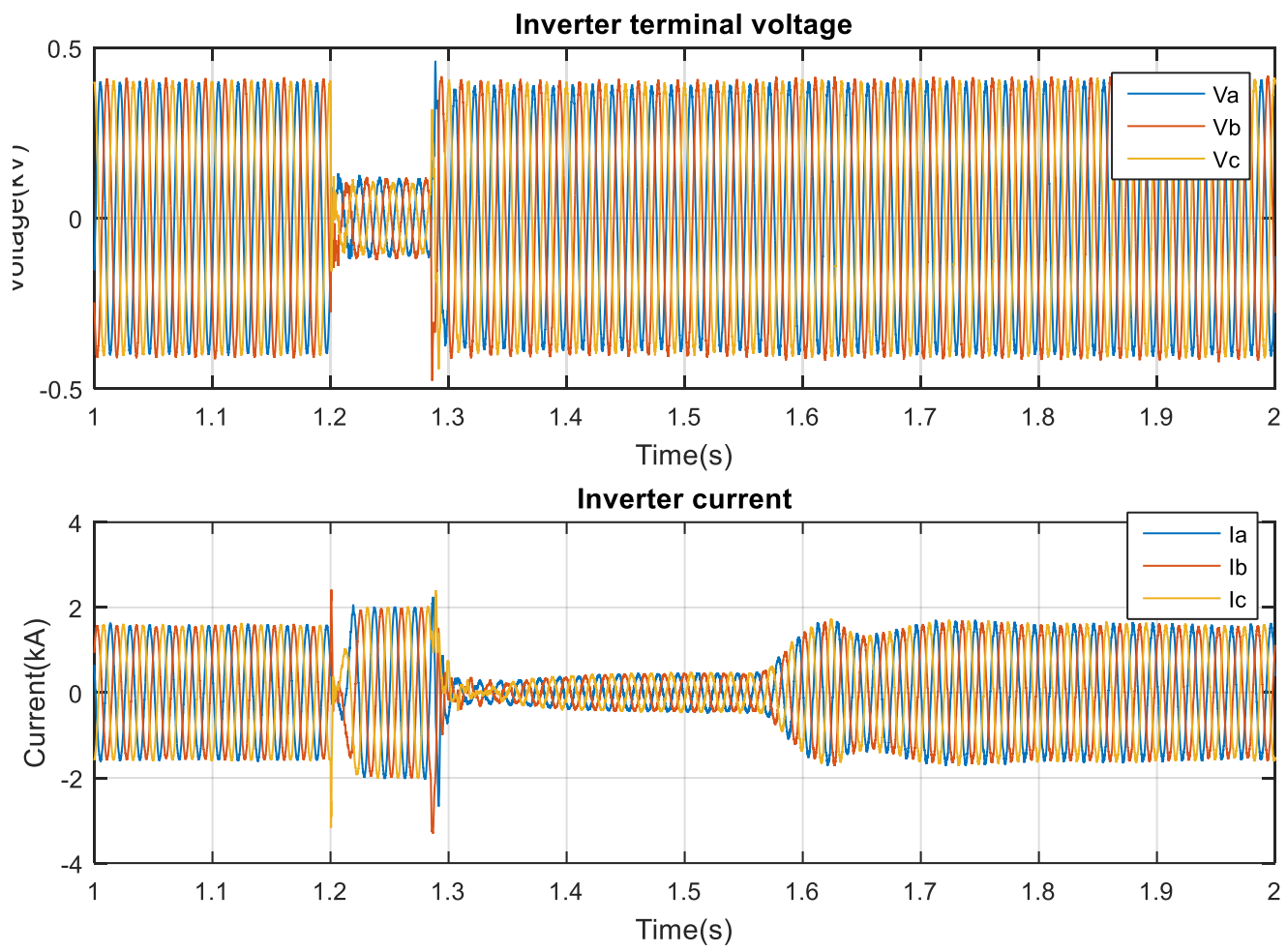

Figure 121 Voltage and currents for case 3 

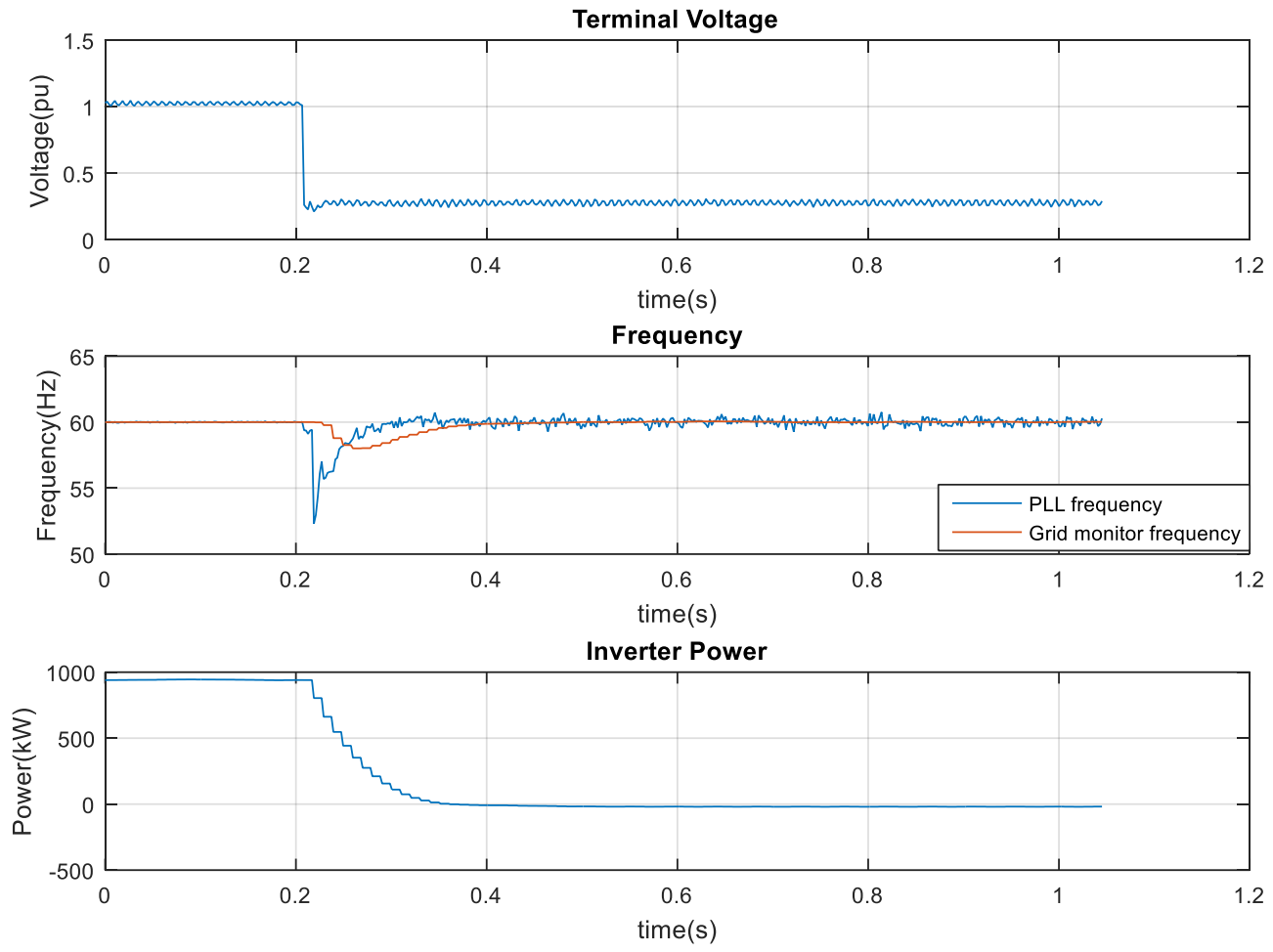

Figure 122 Inverter measurements for case4
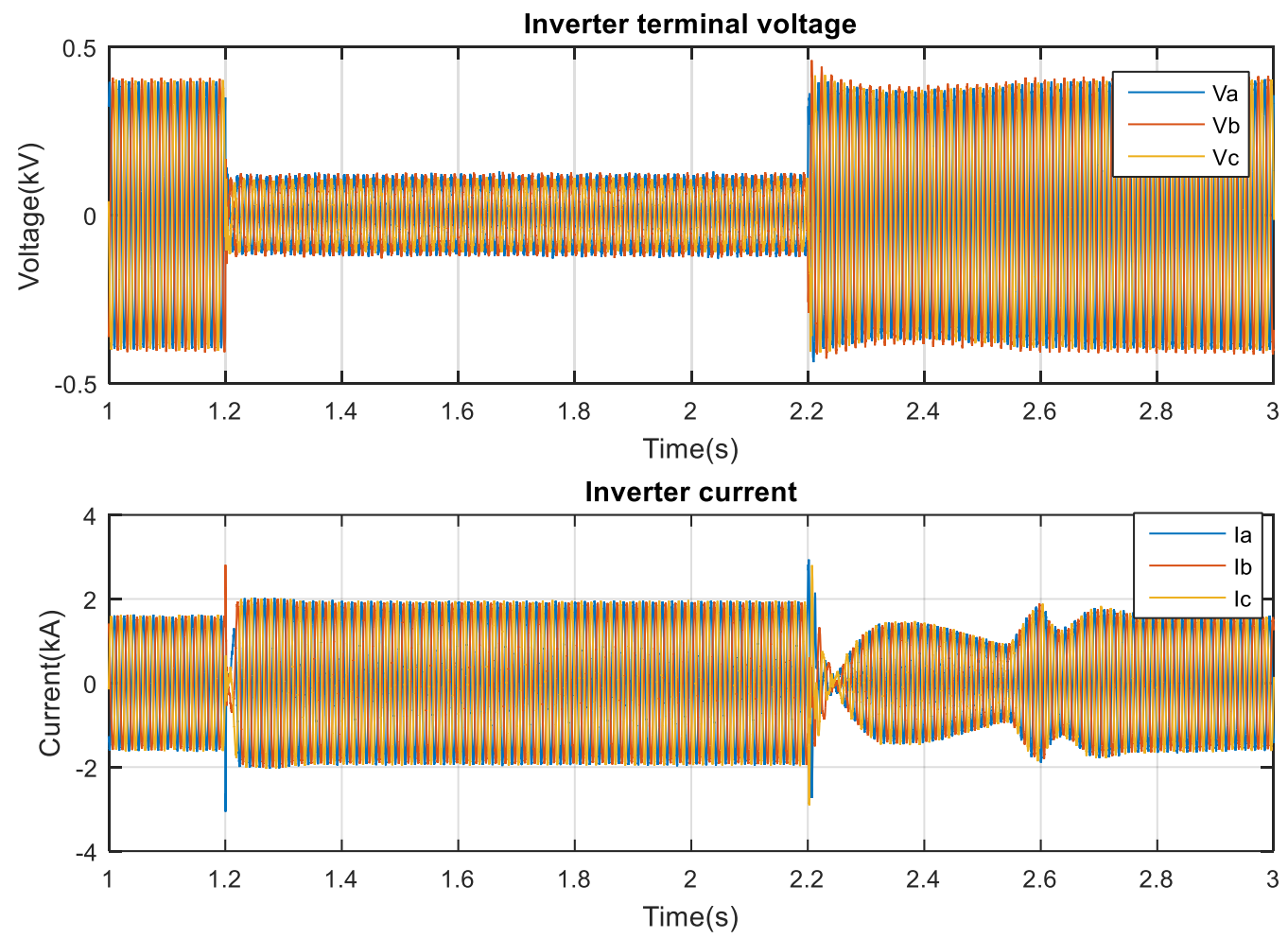

Figure 123 Voltage and currents for case4 

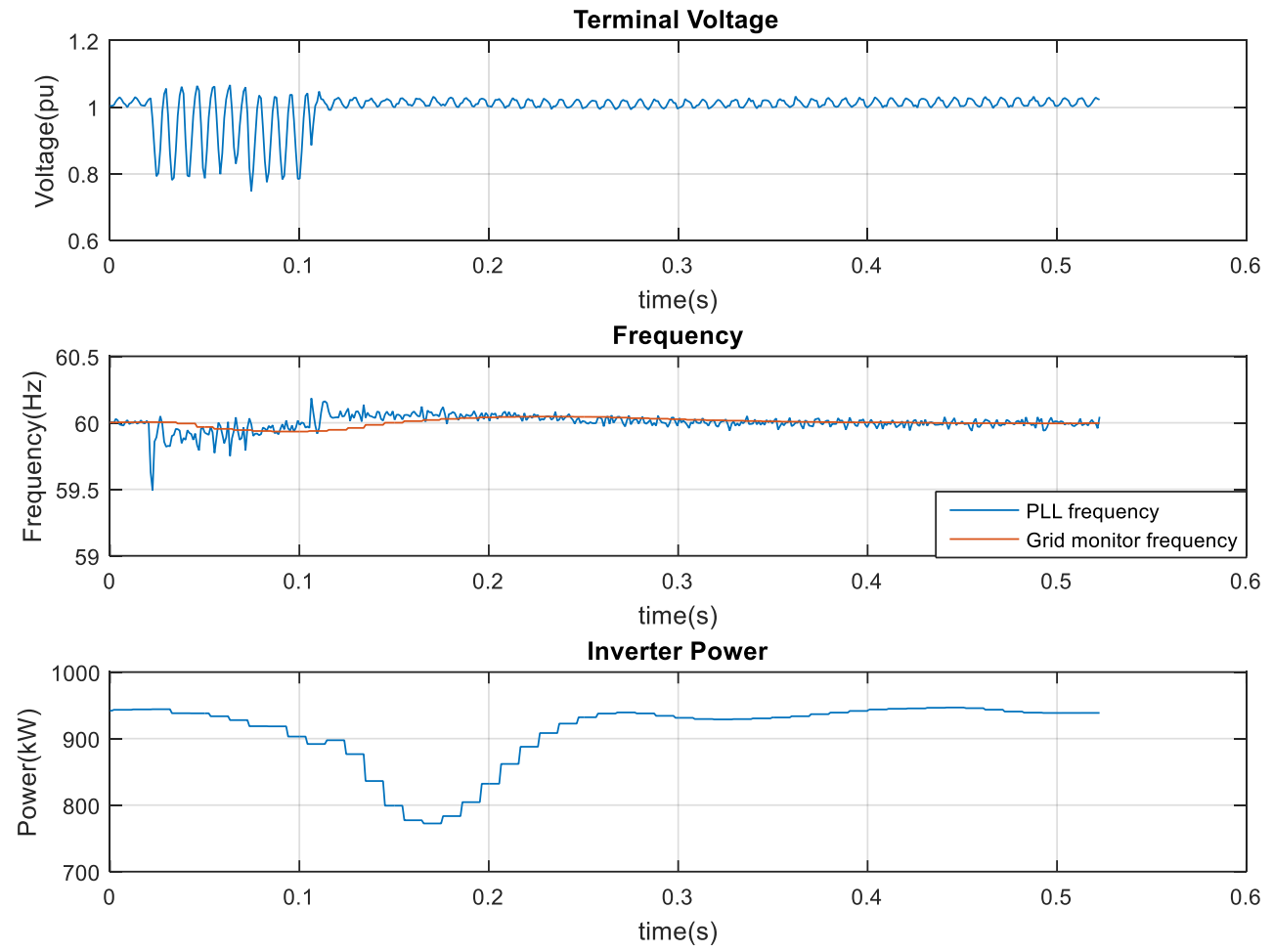

Figure 124 Inverter measurements for case5
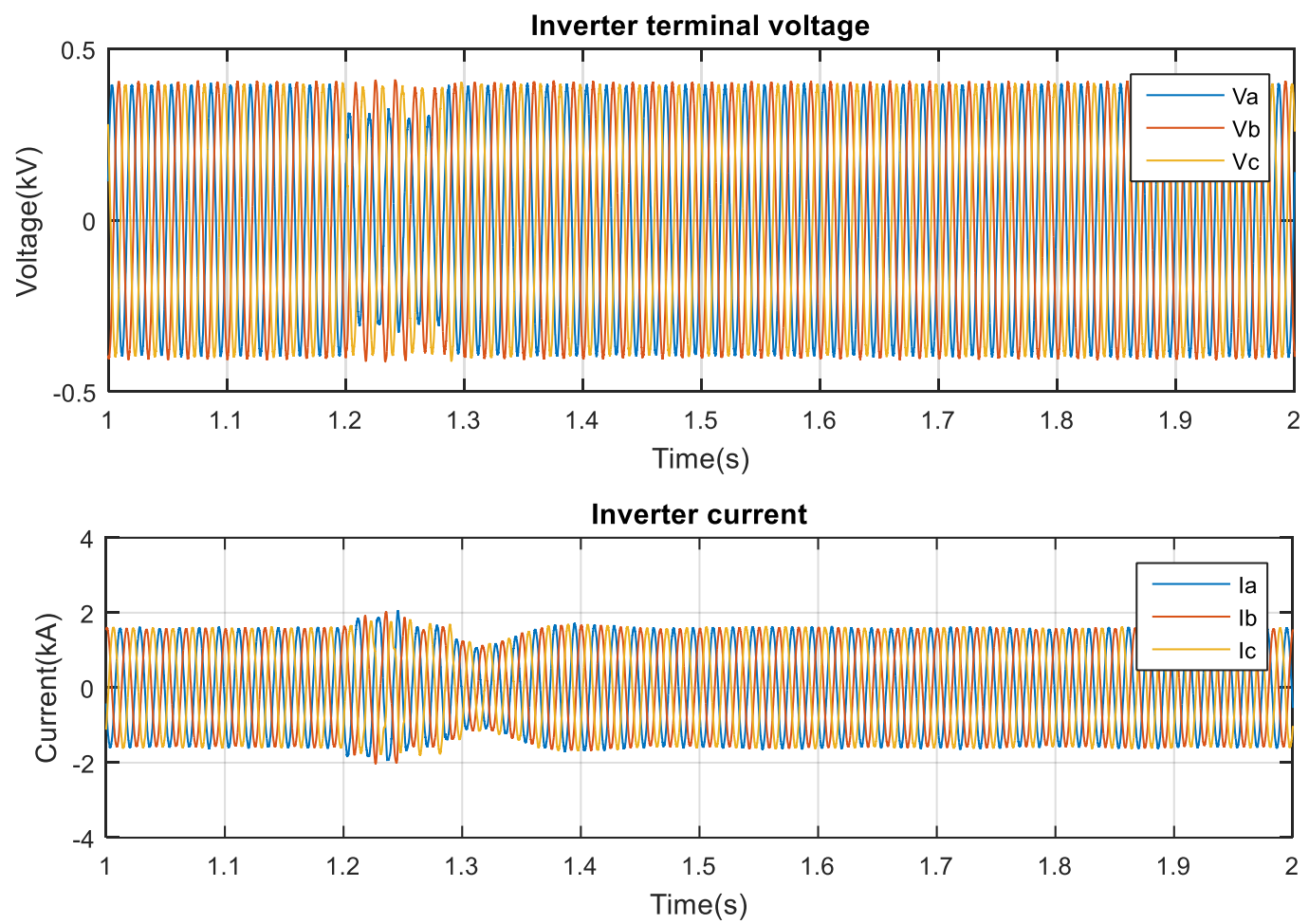

Figure 125 Voltage and currents for case5 


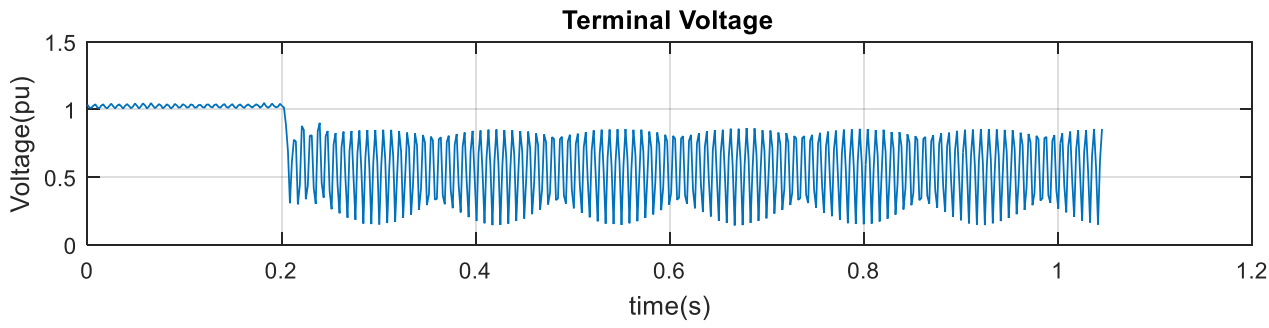

Frequency

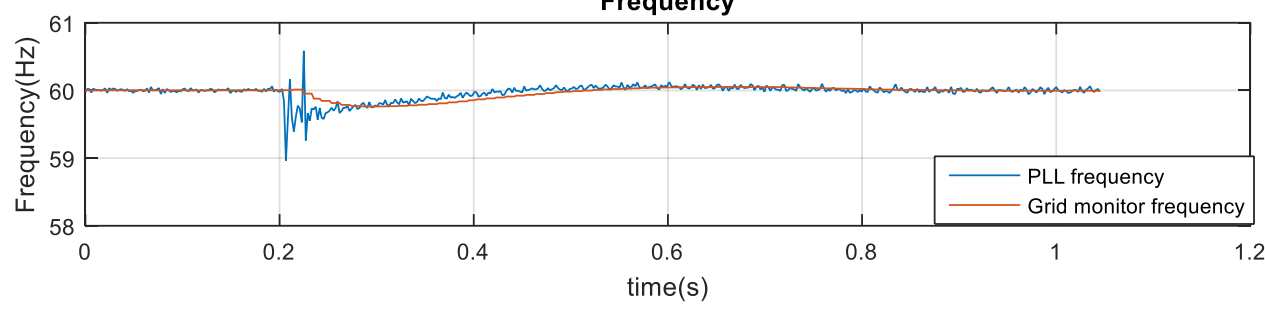

Inverter Power

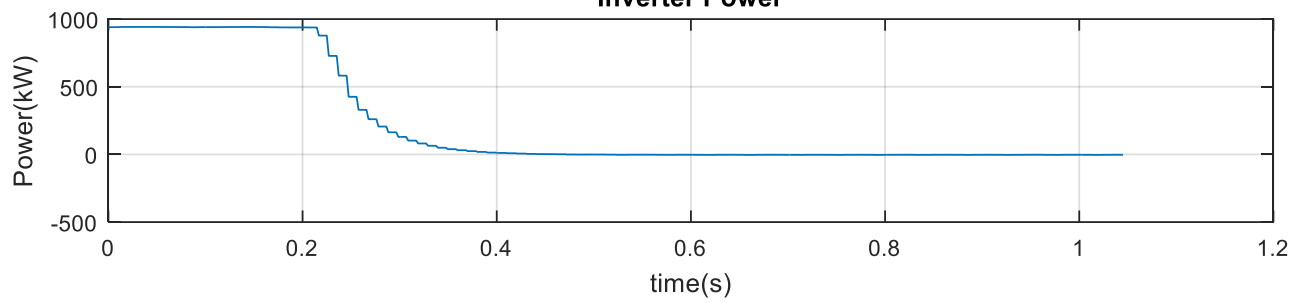

Figure 126 Inverter measurements for case6
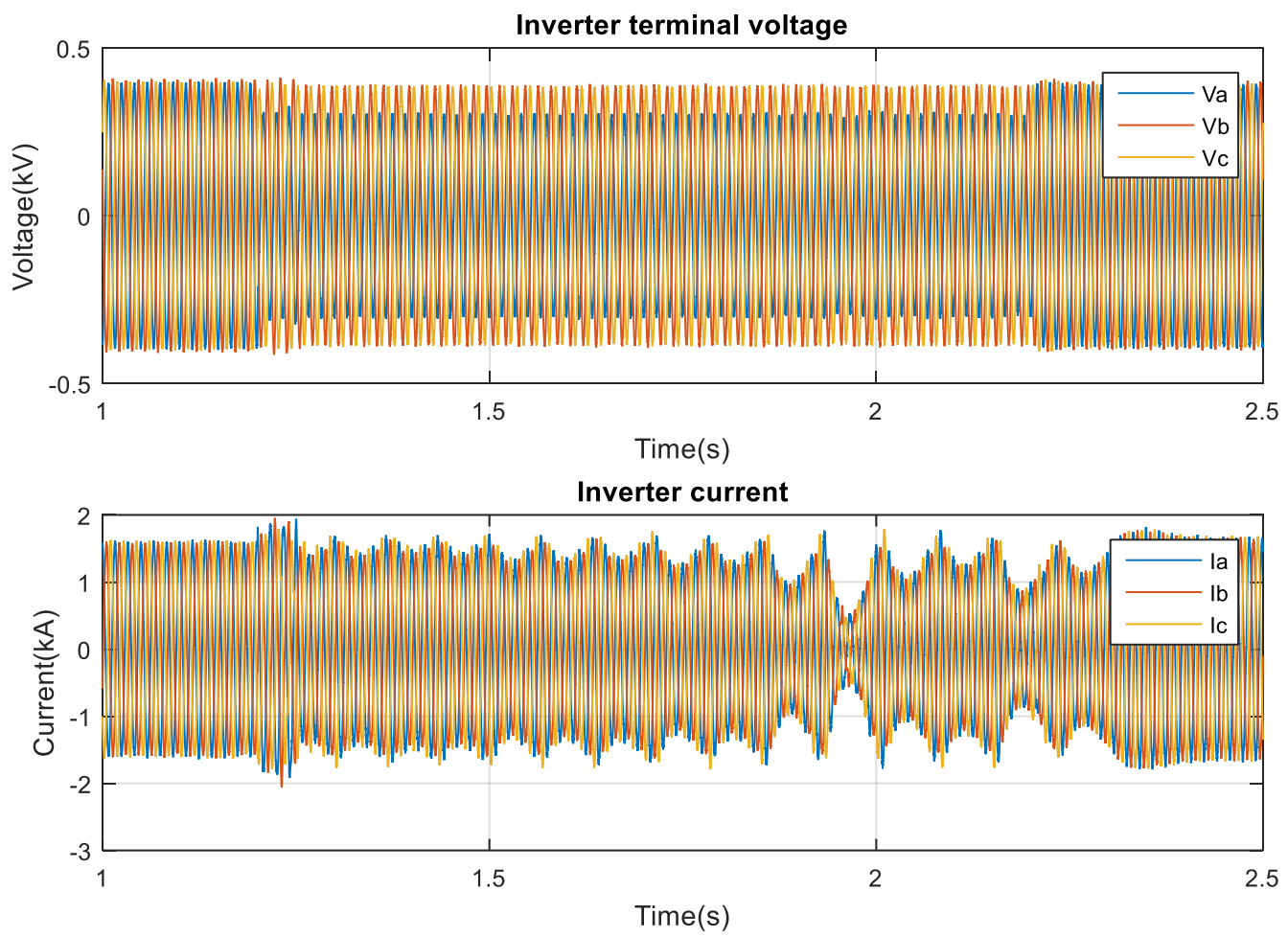

Figure 127 Voltage and currents for case6 

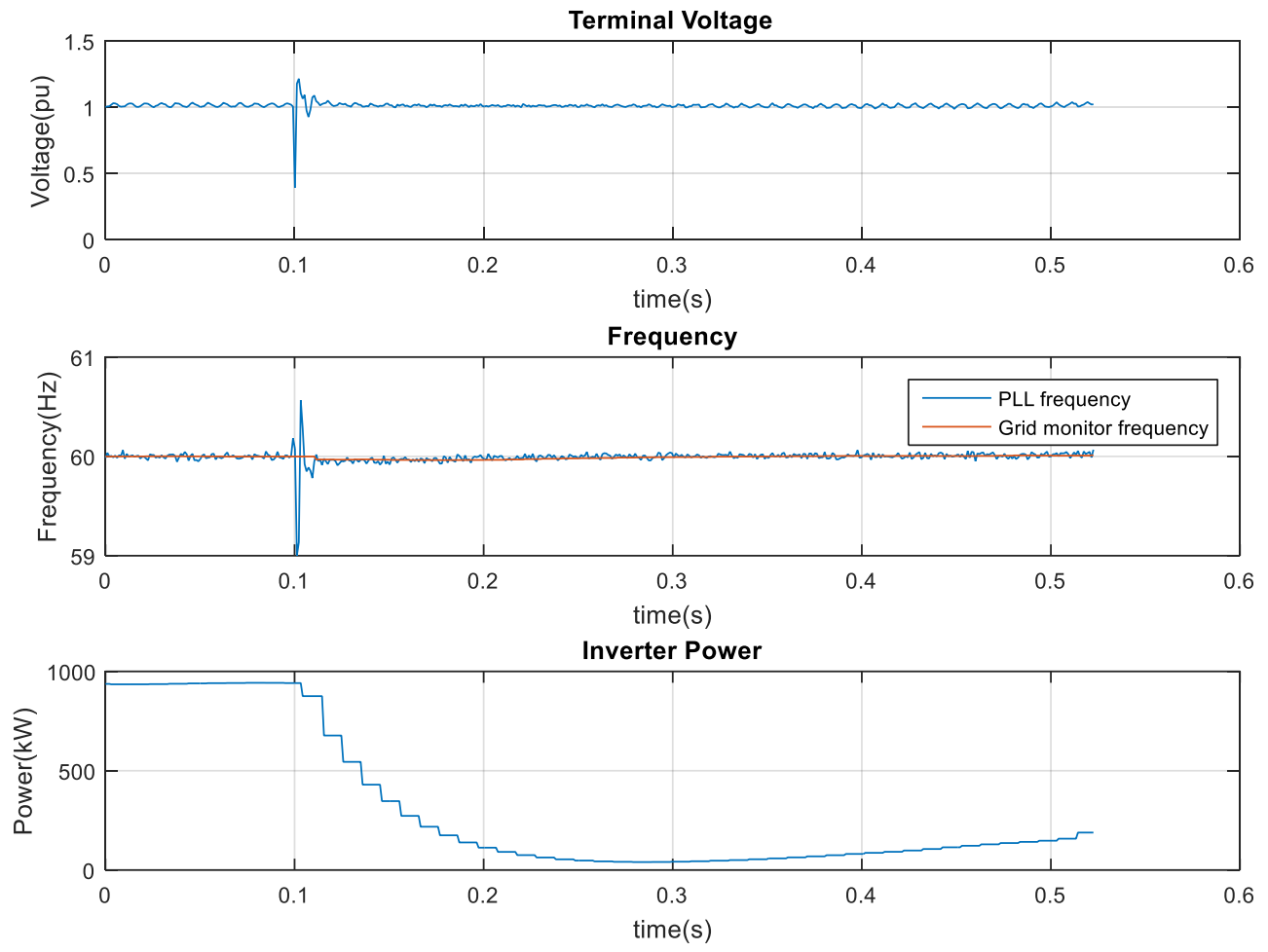

Figure 128 Inverter measurements for case7
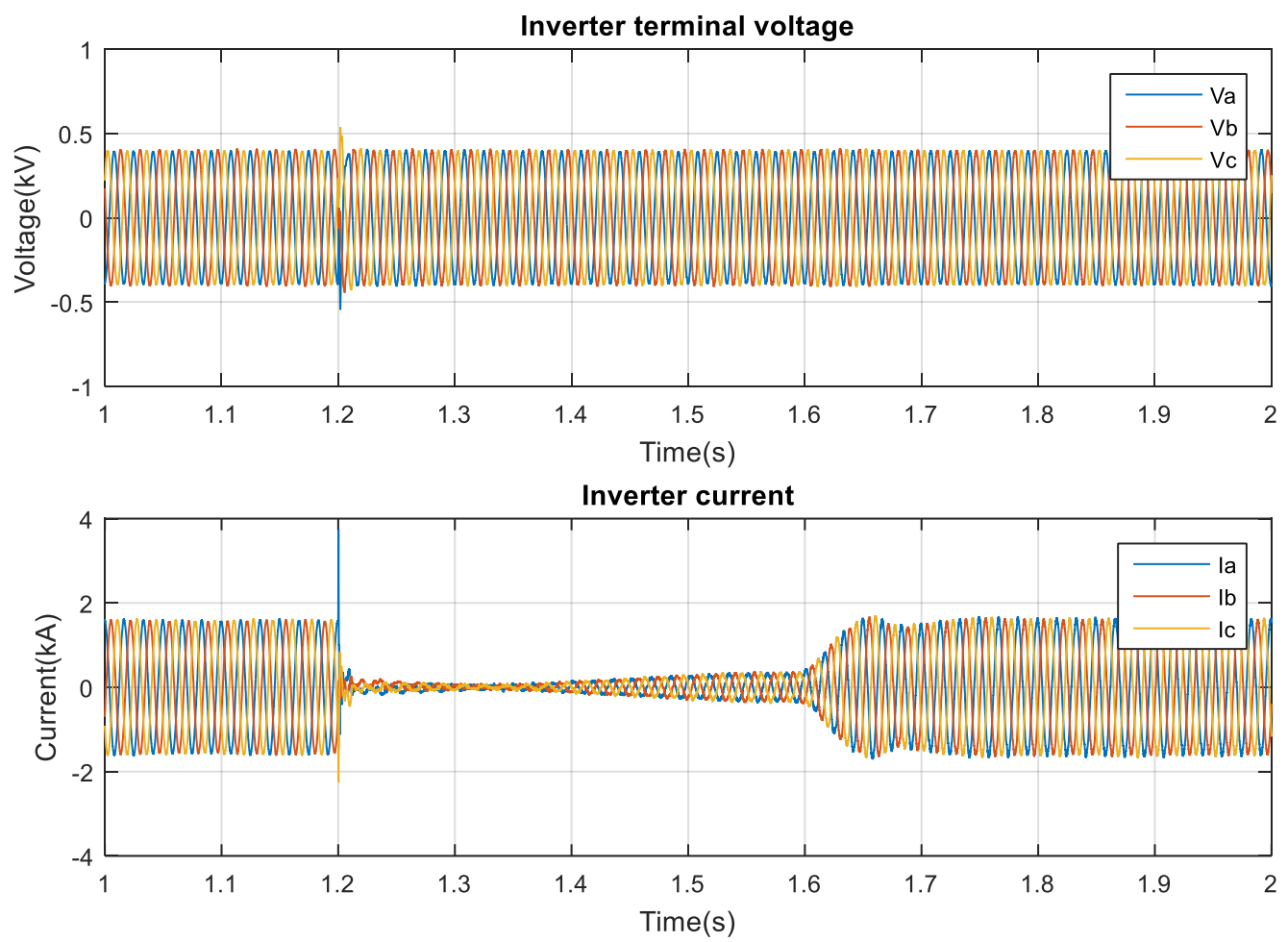

Figure 129 Voltage and currents for case7 

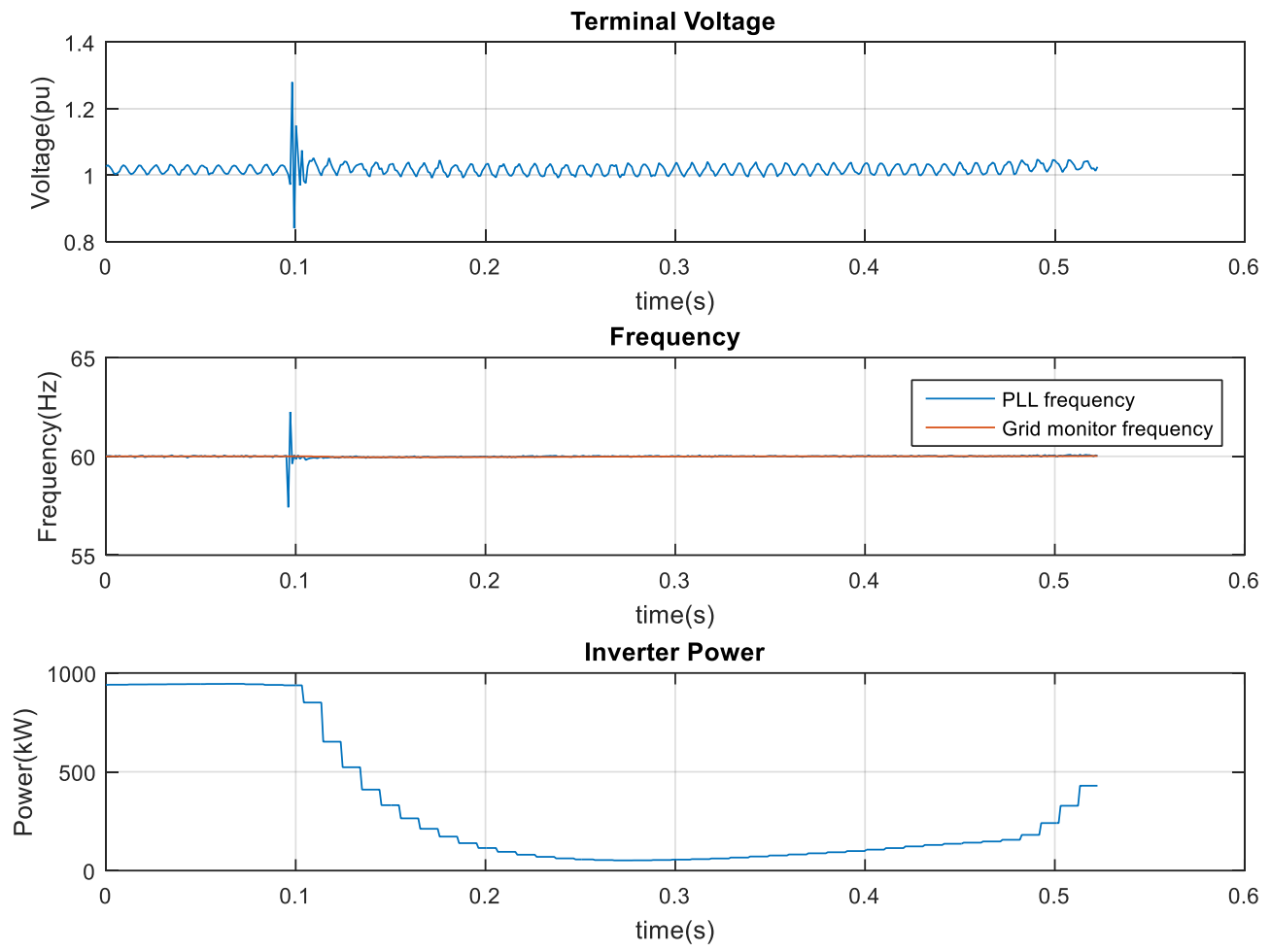

Figure 130 Inverter measurements for case8
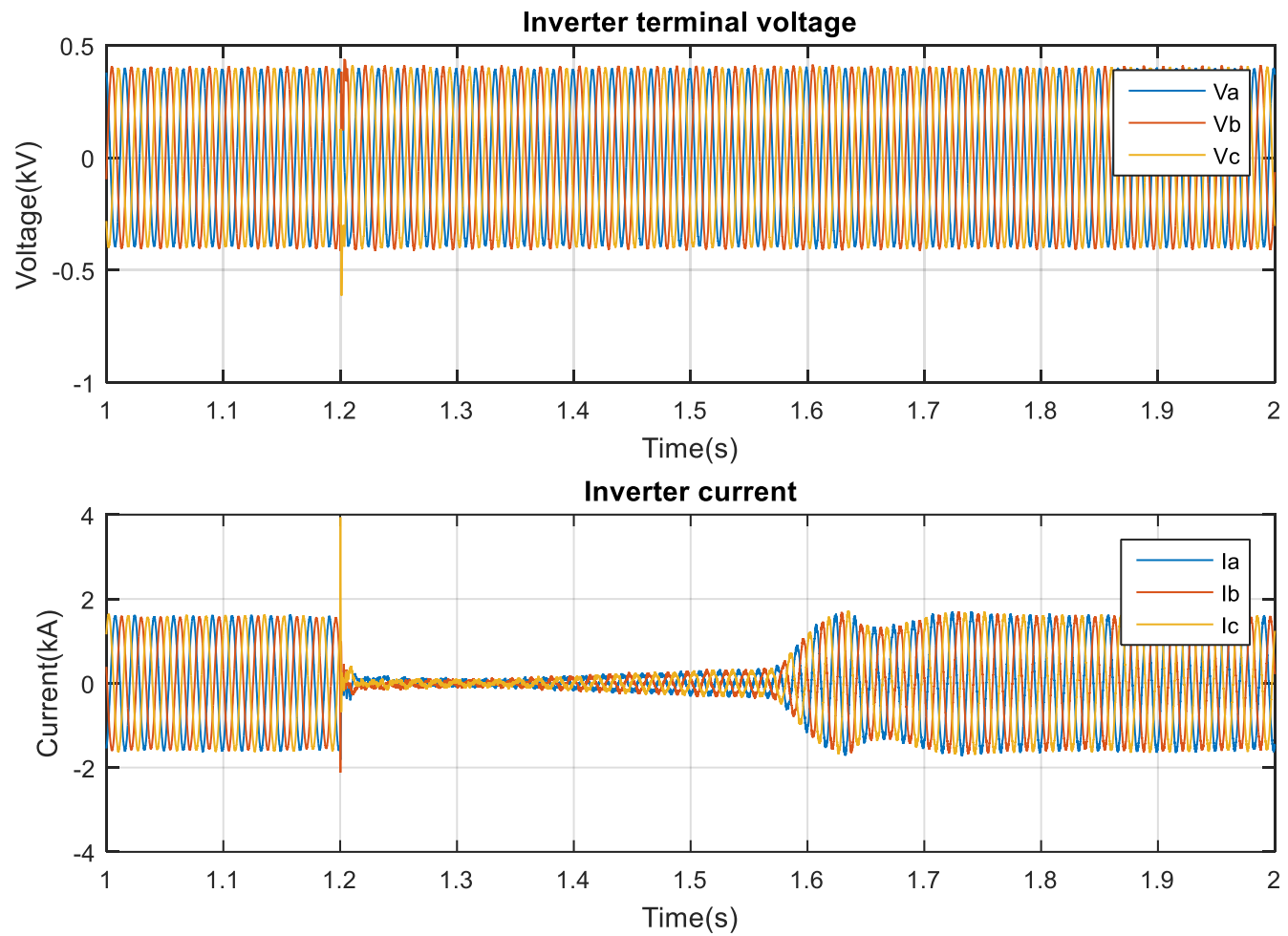

Figure 131 Voltage and currents for case8

For all the cases, we can see from the results above that the faults cause sudden changes in the voltage phase angle and voltage magnitude which can be picked up correctly by the inverter PLL and the inverter 
can ride through the events successfully. Also we noticed that the frequency measurements inside the inverter don't trigger the inverter protection.

\subsubsection{Summary}

The inverter was tested in the IEEE 13 bus system. Different fault scenarios were created to test the inverter performance. The observation is basically the same with the previous tests using playback of generated grid events data.

Under all events, GE inverter does not trip on erroneous frequency measurements. Although the inverter PLL does have transient frequency measurement, it is necessary for the inverter to synchronize with the grid. The inverter does not directly use the PLL outputs to do the protection. Although not tripping, the GE inverter does have a voltage-dependent power reduction and a ramp feature after the grid recovery which was required by the grid code when the control was developed. 


\section{SIGNIFICANT ACCOMPLISHMENTS AND CONCLUSIONS}

Overall, this project, for the first time, proposes a two-step approach to achieve reliable grid measurements. Smooth integration of utility-scale PV is critical to SETO's 2030 target. The 700 MW utility-scale PV trip event revealed the vulnerability of PV inverters to the inaccurate frequency measurement, and the existing corrective action cannot fully solve the problem. By developing a faulttolerant frequency algorithm, this project provides a fundamental solution to mitigate the reliability risk caused by incorrect frequency measurement.

To be specific, the state-of-the-art and commercially wide used measurement technologies have been reviewed first, and the frequency measurement problem of these technologies during the system transient faults has been investigated and tested. The tests verified that they fail to provide reliable measurements during the system transients, like what happened during the Blue Cut Fire event. The tests discovered that the existing technologies failed because they assume the power grid waveforms are always continuous in the sinusoidal format. These assumptions are true when the power system operates normally without a transient fault. However, during a system transient fault, the grid waveforms are usually highly distorted and could have discontinuity due to phase angle jump (or magnitude step change), and unfortunately the transient period cannot be modeled or monitored by these measurement technologies, which results in large measurement errors. Based on the analysis, this project has developed a fault-tolerant frequency measurement technology that can overcome this problem.

First, the team has developed a grid transient detector for the fault-tolerant frequency measurement algorithm, which state-of-the-art measurement technologies does not have. The detector can detect the sudden change of grid signals like phase-jump by using the point-on-wave based approach. Different levels of phase-jump have been used to test the detector. In the meantime, the detector can ride through the signal distortions that will not cause significant measurement errors such as noise and harmonic distortions. High level of noise and harmonics have been tested to verify the performance of the detector. In brief, by detecting grid signals' transients and providing the detection information to the following frequency estimator, the detector is a key component to address the measurement uncertainties under system transients.

Second, based on the developed grid transient detector, the team has developed an intelligent faulttolerant frequency estimator, which is adaptive to the grid transient events. The factors that impact the fault threshold have been studied, and the method to determine the threshold has been presented. It has been found that the threshold value is nearly proportional to the sampling frequency. Signals of different frequency with different levels of noise, harmonics, voltage sag, and phase-jump have been used to test the proposed fault-tolerant frequency estimator. The frequency measurement errors are within $0.5 \mathrm{~Hz}$ in two cycles and $0.05 \mathrm{~Hz}$ in 6 cycles following a phase-jump event, and fully meet milestone 2. Overall, during the system transient events, the whole estimator goes through four stages from frequency lock, extreme fast frequency estimation, fast frequency estimation, and classical frequency estimation. This multiple stage approach has two advantages over classical approach: 1) mitigate frequency estimation errors during system transients; 2) provide frequency measurements in a timely manner after faults by using the extreme fast and fast frequency estimator. Additionally, this approach also maintains the highlevel measurement accuracies as this approach will recover to a classical algorithm when the system transients end.

Further, the team has performed the algorithm evaluation from two aspects: dependability and security. The two tests demonstrated the algorithm can detect system transient faults and mitigate measurement errors, and do not mistakenly response to the system distortions that will not cause large measurement errors. In addition, the tests have been also performed in RTDS by GE Global Research. The two tests 
will give user confidence that the algorithm can provide reliable measurements during system transient faults, which cannot be provided by state-of-the-art algorithms.

Then, the team has analyzed the computational complexity of the new algorithm and performed computation time test on the new algorithm. The analysis and test verified the low computation cost of the algorithm, which is critical for widely use. Moreover, a low-cost measurement instrument was developed, and the new algorithm was implemented on the instrument. The instrument can run in real-time and provide fault-tolerant measurements. The successful implementation of the new algorithm on low-cost hardware further demonstrated the low computation cost of the new algorithm.

The developed fault-tolerant measurement technology has been tested thoroughly under different steadystate, dynamic, and transient conditions to verify its measurement accuracy, security, and dependability, thus demonstrating the measurements provided by the technology are trustworthy. Further, a real-time fault-tolerant monitor has been developed, built and tested using the highly accurate 6135A/PMUCAL Phasor Measurement Unit Calibration System. The hardware test justified the measurement performance. More importantly, the hardware is a low-cost platform, and it verified the developed method has low computational complexity, which is critical for commercial product adoption. 


\section{INVENTIONS, PATENTS, PUBLICATIONS, AND OTHER RESULTS}

\subsection{INVENTIONS}

Lingwei Zhan, et al., "A fault-tolerant grid frequency estimation method during transients," Invention disclosure.

\subsection{PATENTS}

Lingwei Zhan, et al. "Fault-Tolerant Grid Frequency Measurement Algorithm During Transients", USSN, 17/204,494.

\subsection{PUBLICATIONS}

Zhan, Lingwei; Xiao, Bailu; Li, Fuhua; Yin, He; Yao, Wenxuan; Li, Zhi; Liu, Yilu: 'Faulttolerant grid frequency measurement algorithm during transients', IET Energy Systems Integration, 2020, 2, (3), p. 173-178, DOI: 10.1049/iet-esi.2019.0126.

Fuhua Li, et al., Frequency Computation During Events and Point-on-wave Measurement, 2019 IEEE Power \& Energy Society General Meeting, Atlanta, GA, 2019, pp. 1-5.

J. Zhao, L. Zhan, et al., "Recent Development of Frequency Estimation Methods for Future Smart Grid," in IEEE Open Access Journal of Power and Energy, vol. 7, pp. 354-365, 2020, doi: 10.1109/OAJPE.2020.3029984.

\subsection{PRESENTATIONS}

Lingwei Zhan, "Development of low-cost time synchronized point-on-wave data recorder and fault-tolerant grid frequency measurements technologies," NASPI, April 15-17, 2019, San Diego, CA.

Lingwei Zhan, “Towards High Speed and Fault-tolerant Grid Measurements”, WECC JSIS Meeting, November 14-15, Salt Lake City, Utah.

Fuhua Li, "Frequency Computation During Events and Point-on-wave Measurement", 2019 IEEE Power \& Energy Society General Meeting, Atlanta, GA, 2019, pp. 1-5. 


\section{PATH FORWARD}

Field Data Validation and Demonstration: Field validation and demonstration is one step forward towards commercialization. First, we can collaborate with utilities by testing the new measurement technology with the Digital Fault Recorder (DFR) data, which can provide different types of real system transient faults data. By utilizing large amount of DFR data, the measurement performance of the developed technology can be thoroughly evaluated, and its limitations could also be revealed, which is very useful for further improvement. In the meantime, the validation test may also help discover any other unknown measurement challenge. Further, the developed fault-tolerant monitor can be deployed in field to continuously collect grid data and monitor the power grid transients.

DER Hardware Demonstration: A critical work towards widely industry adoption is to demonstrate the developed technology on DER system like the solar inverters and perform Hardware in Loop (HIL) testing. As this project is a one-year project and the problem being solved is new, existing hardware test platform, instead of commercial GE's solar inverters, was used in the project period to evaluate the new algorithm. The existing hardware platform has a PMU calibrator that can generate various and accurate test signals, which are critical for performance evaluation. Next step of this project is to develop the algorithm in solar inverters, some companies including GE have shown interests in developing the new algorithm in their products. It should be noted that this work need careful evaluation since measurements are fundamental low-level component for high-level grid control and protection applications, and the new measurement method shall not negatively impact the applications. A good strategy is to implement the new method in parallel with the existing measurement method and two methods perform the measurement in parallel.

Real-time Grid Anomaly Alarming: In fact, the real-time "transient detector" developed in this project can operate separately from the frequency estimator. In other words, the transient detector can operate independently to monitor the occurrence of "transient fault" in real-time. Therefore, it can be applied to monitor grid anomalies, and any other measurements, not limited to frequency measurement, and data analysis applications can use the transient detector data for secure and reliable grid operations. 


\section{REFERENCE}

[1] U.S. Department of Energy, "Maintaining Reliability in the Modern Power System”, December, 2016.

[2] NERC, “1,200 MW Fault Induced Solar Photovoltaic Resource Interruption Disturbance Report”, June, 2017.

[3] A. G. Phadke and J. S. Thorp, Synchronized Phasor Measurements and Their Applications. New York, NY, USA: Springer, 2008.

[4] T. Xia and Y. Liu, 'Single-phase phase angle measurements in electric power systems,' IEEE Trans. Power Syst., vol. 25, no. 2, pp. 844-852, May 2010.

[5] R. Mai, Z. He, L. Fu, B. Kirby, and Z. Bo, 'A dynamic synchrophasor estimation algorithm for online application,' IEEE Trans. Power Del., vol. 25, no. 2, pp. 570-578, Apr. 2010.

[6] I. Kamwa, A. K. Pradhan and G. Joos, 'Adaptive Phasor and Frequency-Tracking Schemes for WideArea Protection and Control,' in IEEE Transactions on Power Delivery, vol. 26, no. 2, pp. 744-753, April 2011.

[7] I. Kamwa, S. R. Samantaray and G. Joos, 'Wide Frequency Range Adaptive Phasor and Frequency PMU Algorithms,' in IEEE Transactions on Smart Grid, vol. 5, no.2, pp. 569-579, March 2014.

[8] D. Macii, D. Petri and A. Zorat, 'Accuracy Analysis and Enhancement of DFTBased Synchrophasor Estimators in Off-Nominal Conditions,' in IEEE Transactions on Instrumentation and Measurement, vol. 61, no. 10, pp. 2653-2664, Oct. 2012.

[9] W. Premerlani, B. Kasztenny, and M. Adamiak, 'Development and implementation of a synchrophasor estimator capable of measurements under dynamic conditions,' IEEE Trans. Power Del., vol. 23, no. 1, pp. 109-123, Jan. 2008.

[10] P. Romano and M. Paolone, 'Enhanced Interpolated-DFT for Synchrophasor Estimation in FPGAs: Theory, Implementation, and Validation of a PMU Prototype,' in IEEE Transactions on Instrumentation and Measurement, vol. 63, no. 12, pp. 2824-2836, Dec. 2014.

[11] A. Derviskadic, P. Romano and M. Paolone, 'Iterative-Interpolated DFT for Synchrophasor Estimation: A Single Algorithm for P- and M-Class Compliant PMUs,'in IEEE Transactions on Instrumentation and Measurement, vol. 67, no. 3, pp. 547-558, March 2018.

[12] D. Belega and D. Petri, 'Accuracy Analysis of the Multicycle Synchrophasor Estimator Provided by the Interpolated DFT Algorithm,' in IEEE Transactions on Instrumentation and Measurement, vol. 62, no. 5, pp. 942-953, May 2013.

[13] I. Kamwa, S. R. Samantaray, and G. Joos, 'Compliance analysis of PMU algorithms and devices for wide-area stabilizing control of large power systems,' IEEE Trans. Power Syst., vol. 28, no. 2, pp. 1766-1778, May 2013. 
[14] A. J. Roscoe, I. F. Abdulhadi and G. M. Burt, 'P andM Class Phasor Measurement Unit Algorithms Using Adaptive Cascaded Filters,' in IEEE Transactions on Power Delivery, vol. 28, no. 3, pp. 14471459, July 2013.

[15] A. J. Roscoe, B. Dickerson and K. E. Martin, 'Filter Design Masks for C37.118.1a Compliant Frequency-Tracking and Fixed-Filter M-Class Phasor Measurement Units,' in IEEE Transactions on Instrumentation and Measurement, vol. 64, no.8, pp. 2096-2107, Aug. 2015.

[16] L. Zhan, Y. Liu and Y. Liu, 'A Clarke Transformation-Based DFT Phasor and Frequency Algorithm for Wide Frequency Range,' in IEEE Transactions on Smart Grid, vol. 9, no. 1, pp. 67-77, Jan. 2018.

[17] L. Zhan, Y. Liu, J. Culliss, J. Zhao and Y. Liu, 'Dynamic Single-Phase Synchronized Phase and Frequency Estimation at the Distribution Level,' in IEEE Transactions on Smart Grid, vol. 6, no. 4, pp. 2013-2022, July 2015.

[18] J. A. de la O Serna, 'Dynamic phasor estimates for power system oscillations,' IEEE Trans. Instrum. Meas., vol. 56, no. 5, pp. 1648-1657, Oct. 2007.

[19] J. A. de la O Serna, 'Dynamic phasor estimates for power system oscillations and transient detection,' in Proc. IEEE Power Eng. Soc. Gen. Meeting, Montreal, QC, Canada, 2006, p. 7.

[20] M. A. Platas-Garza and J. A. de la O Serna, 'Dynamic phasor and frequency estimates through maximally flat differentiators,' IEEE Trans. Instrum. Meas., vol. 59, no. 7, pp. 1803-1811, Jul. 2010.

[21] L. Zhan and Y. Liu, 'Improved WLS-TF algorithm for dynamic synchronized angle and frequency estimation,' in Proc. IEEE Power Energy Soc. Gen. Meeting, National Harbor, MD, USA, 2014, pp. 15.

[22] IEEE Standard for Synchrophasor Measurements for Power Systems," in IEEE Std C37.118.12011 (Revision of IEEE Std C37.118-2005), vol., no., pp.1-61, Dec. 282011.

[23] L. Zhan, Jianyang Zhao, J. Culliss, Y. Liu, Y. Liu and Shengyou Gao, "Universal Grid Analyzer design and development," 2015 IEEE Power \& Energy Society General Meeting, Denver, CO, 2015, pp. 1-5.

[24] Á. Espín-Delgado, J. R. Camarillo-Peñaranda and G. Ramos, "Characterization of Phase-Angle Jump in Radial Systems Using Incremental Voltage Phasors," IEEE Transactions on Industry Applications, vol. 55, no. 2, pp. 1117-1125, Mar. 2019.

[25] IEEE Standard for Synchrophasor Measurements for Power Systems -- Amendment 1: Modification of Selected Performance Requirements," in IEEE Std C37.118.1a-2014 (Amendment to IEEE Std C37.118.1-2011), vol., no., pp.1-25, April 302014. 


\section{APPENDIX A. DEPENDABILITY TEST}
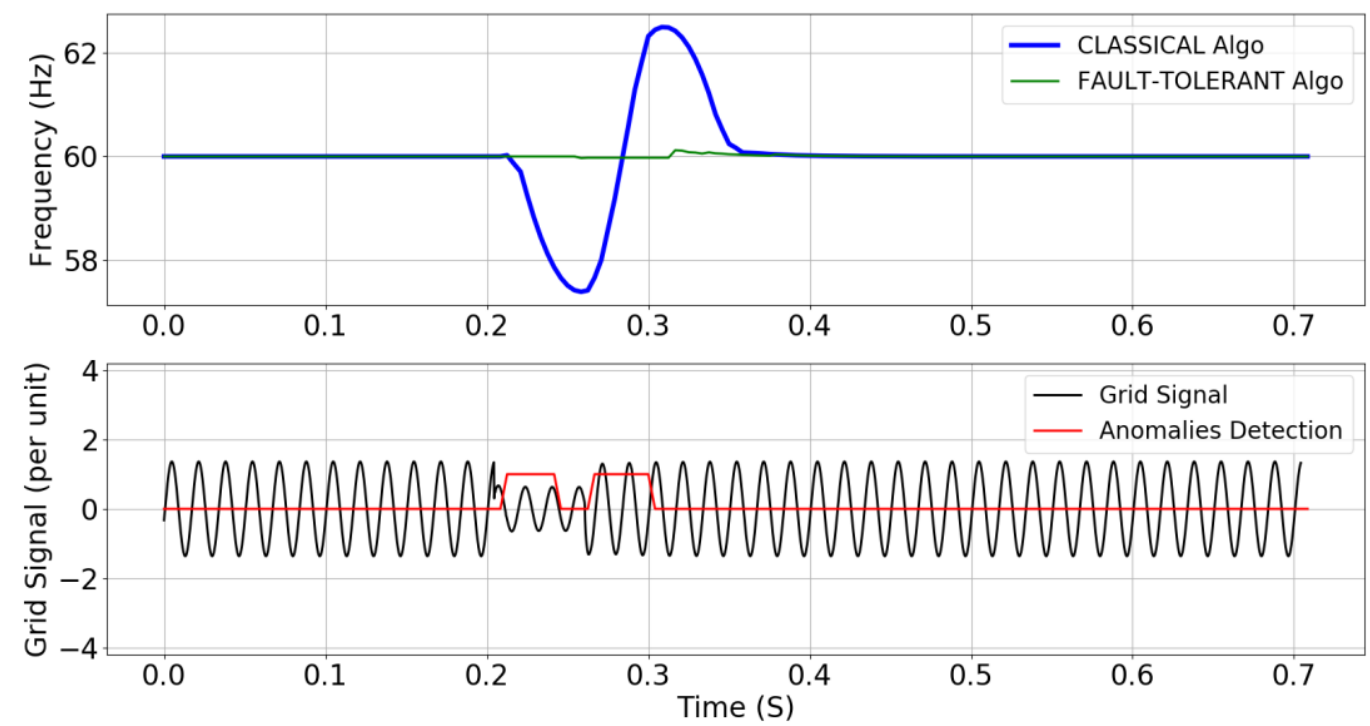

Figure 132 time domain result - case 2
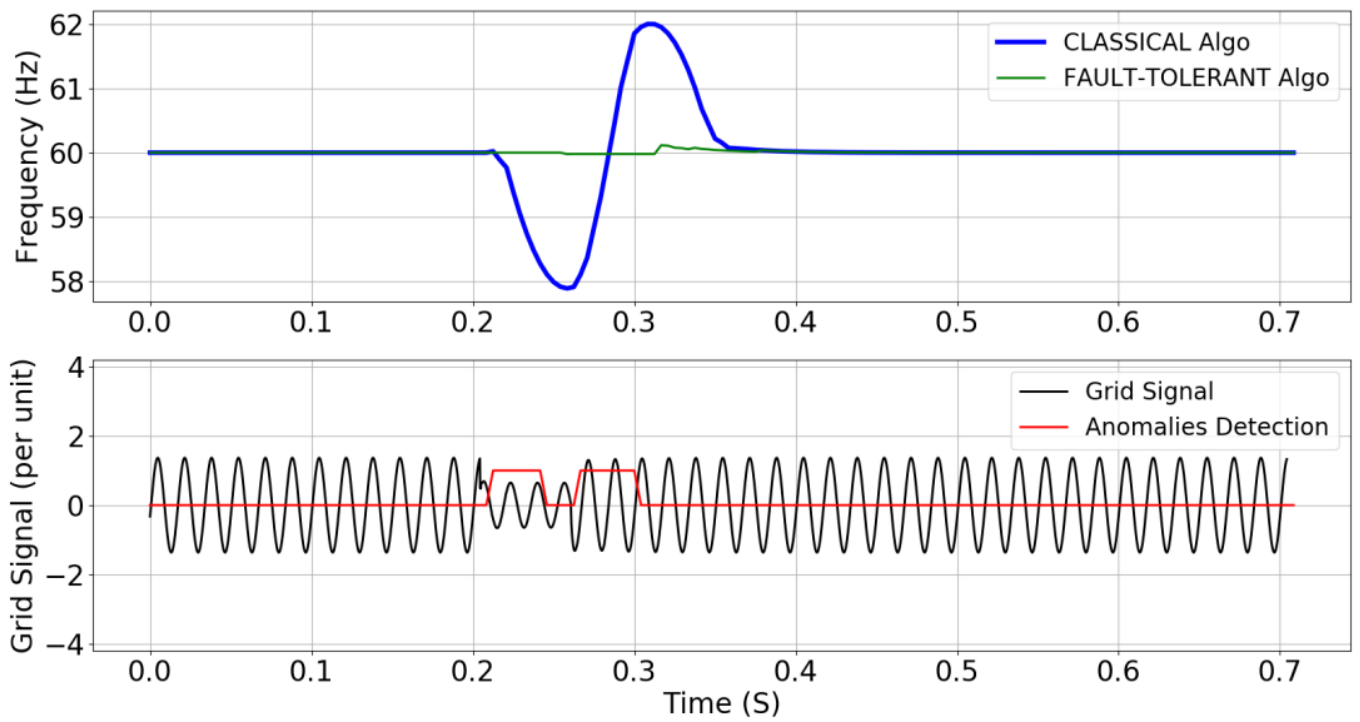

Figure 133 time domain result - case 3 

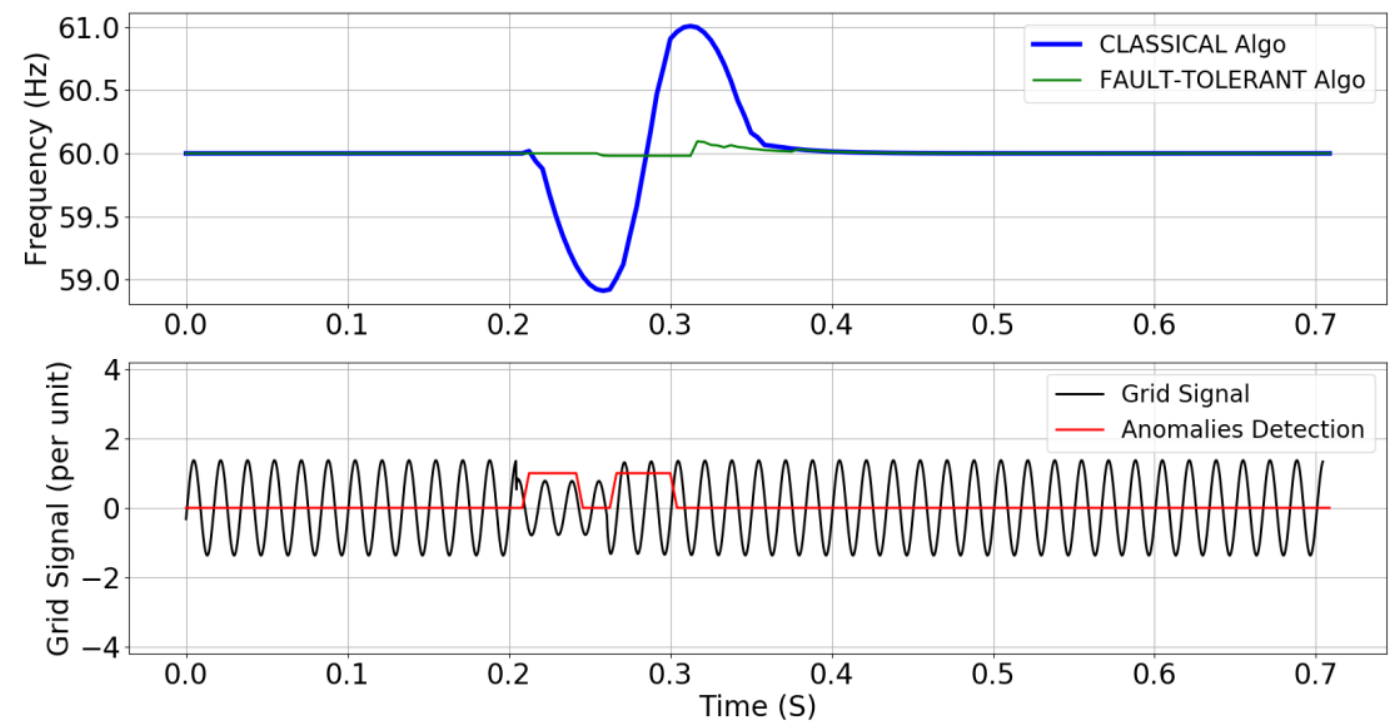

Figure 134 time domain result - case 4
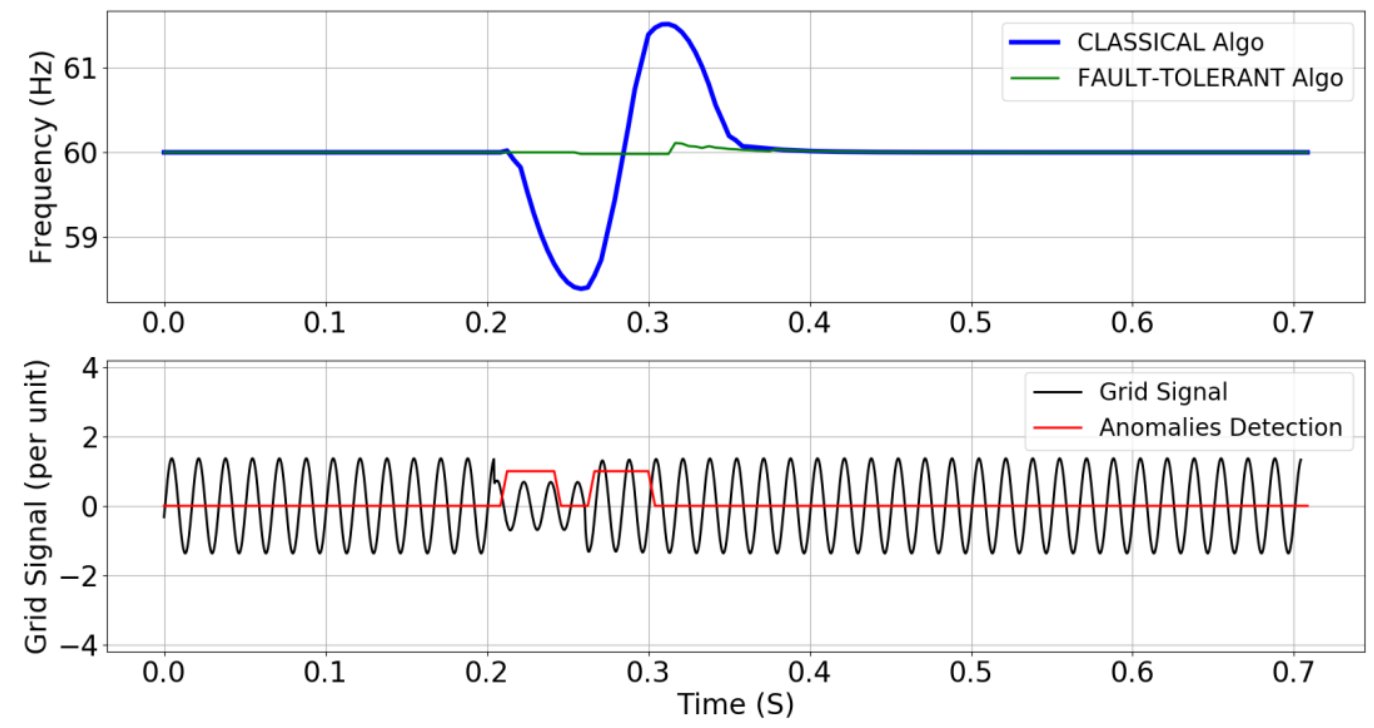

Figure 135 time domain result - case 5 

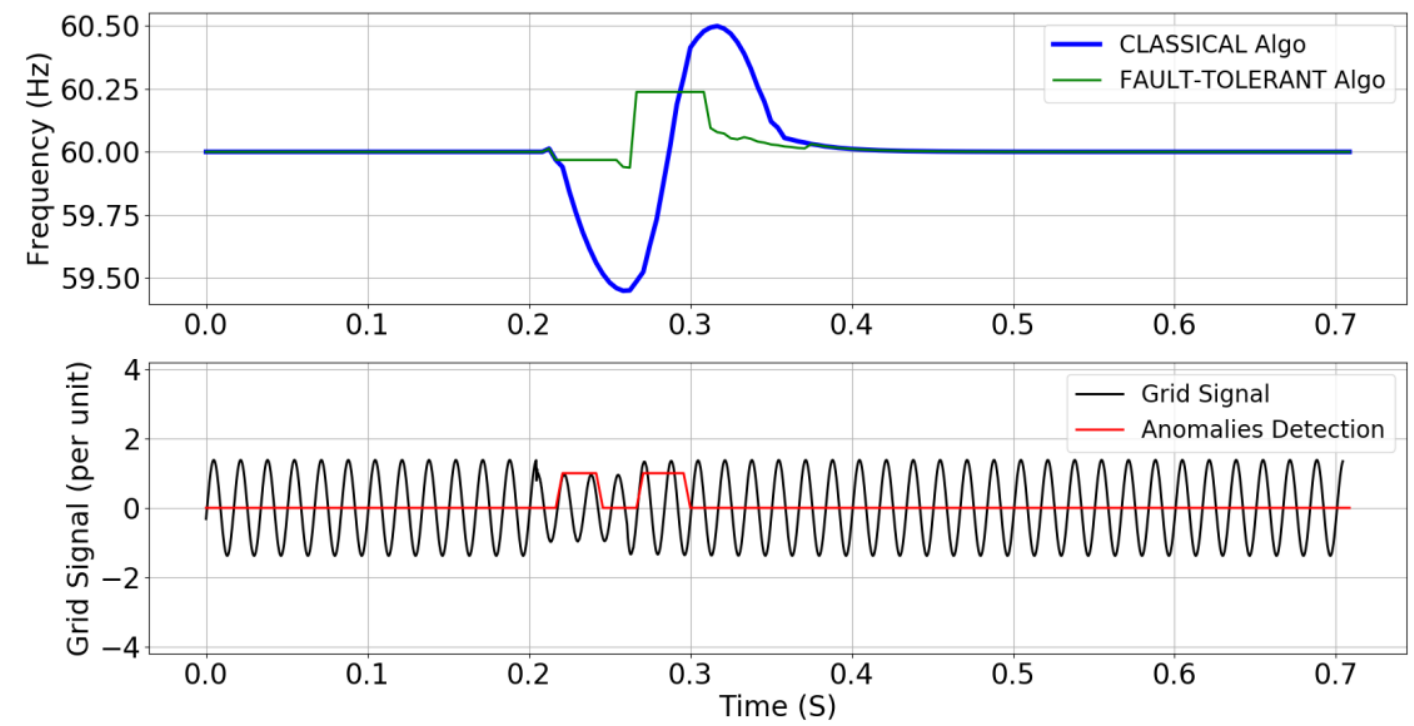

Figure 136 time domain result - case 6
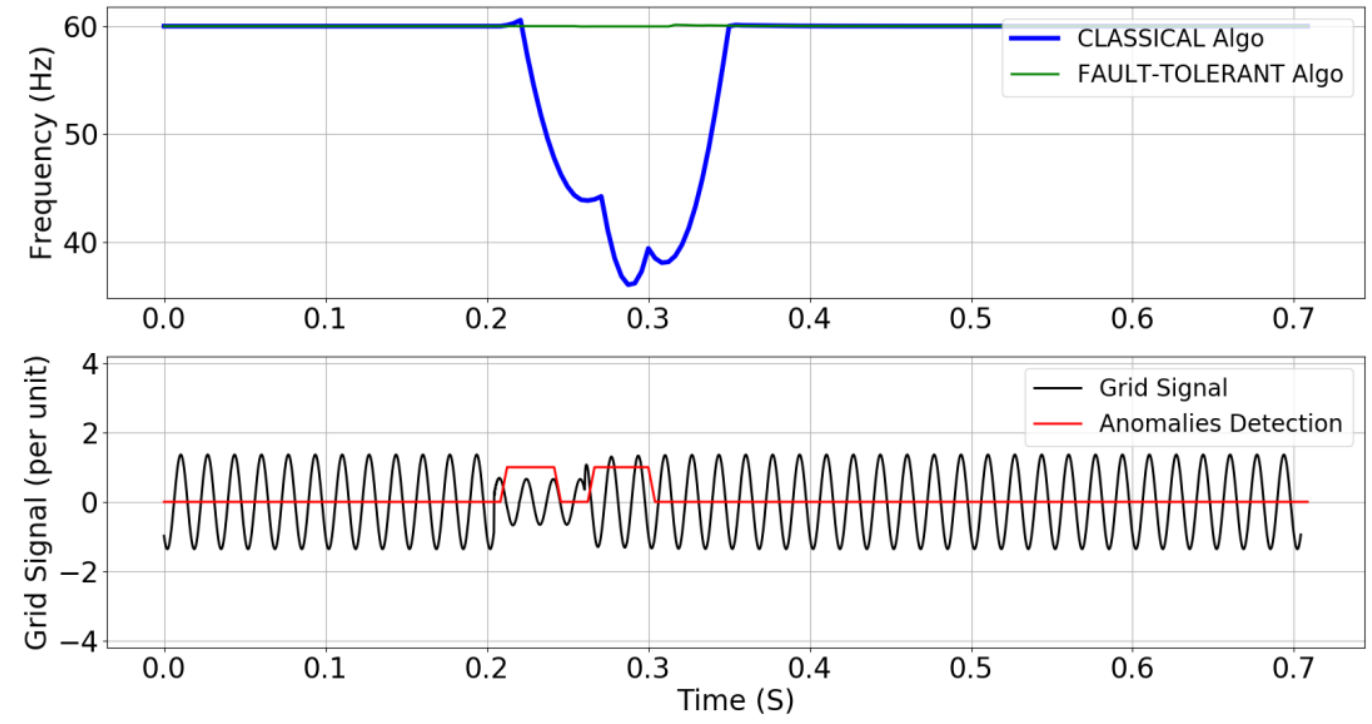

Figure 137 time domain result - case 7 

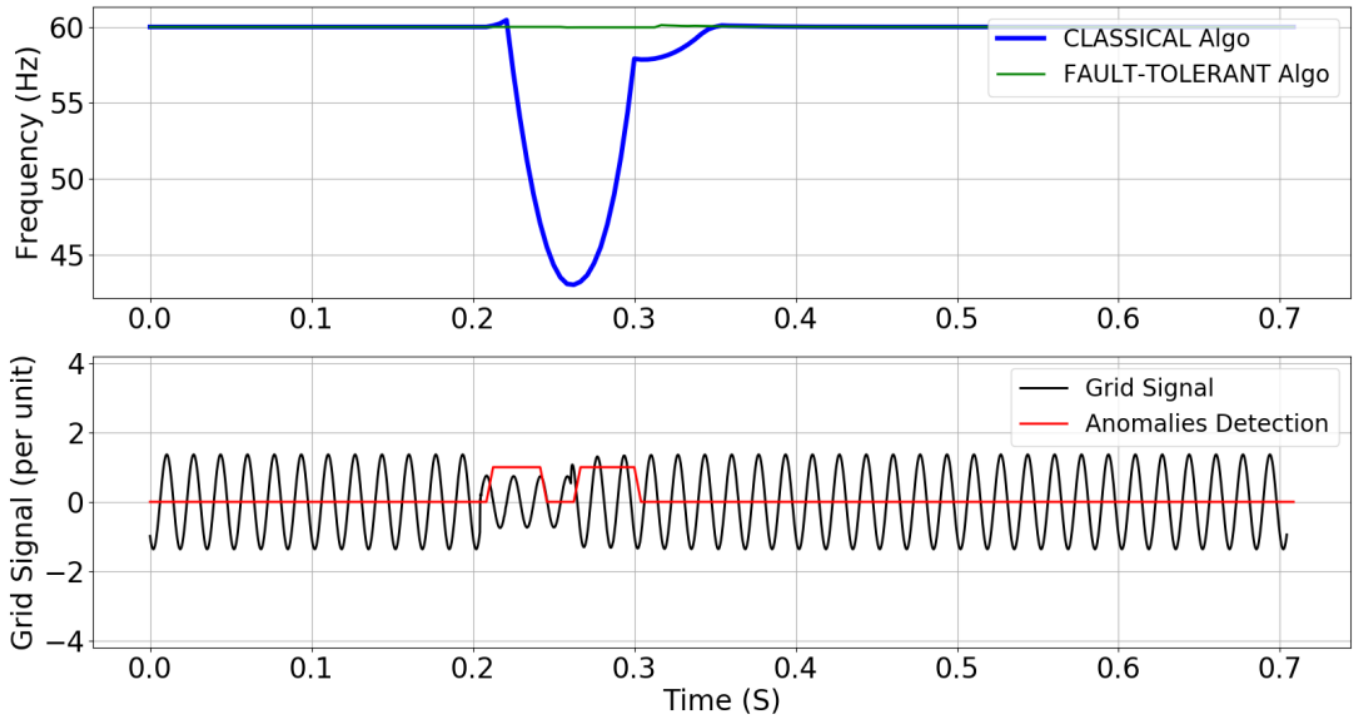

Figure 138 time domain result - case 8
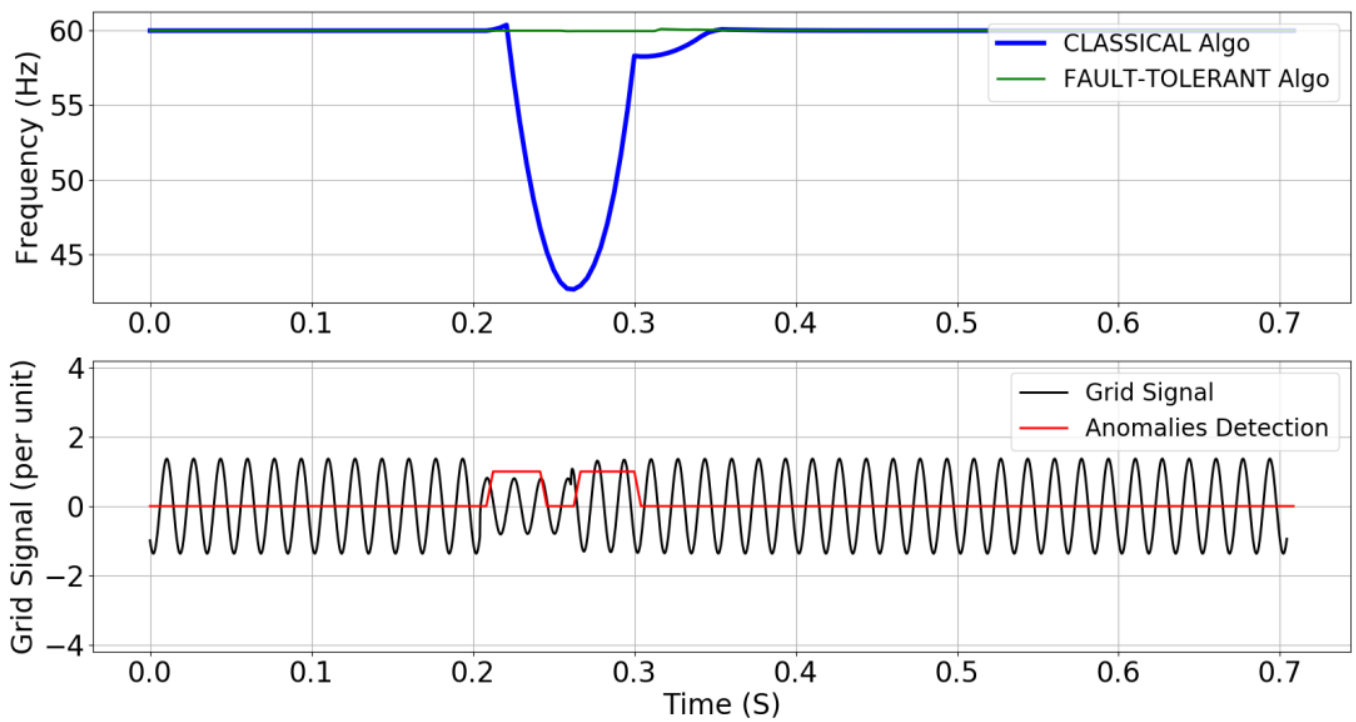

Figure 139 time domain result - case 9 

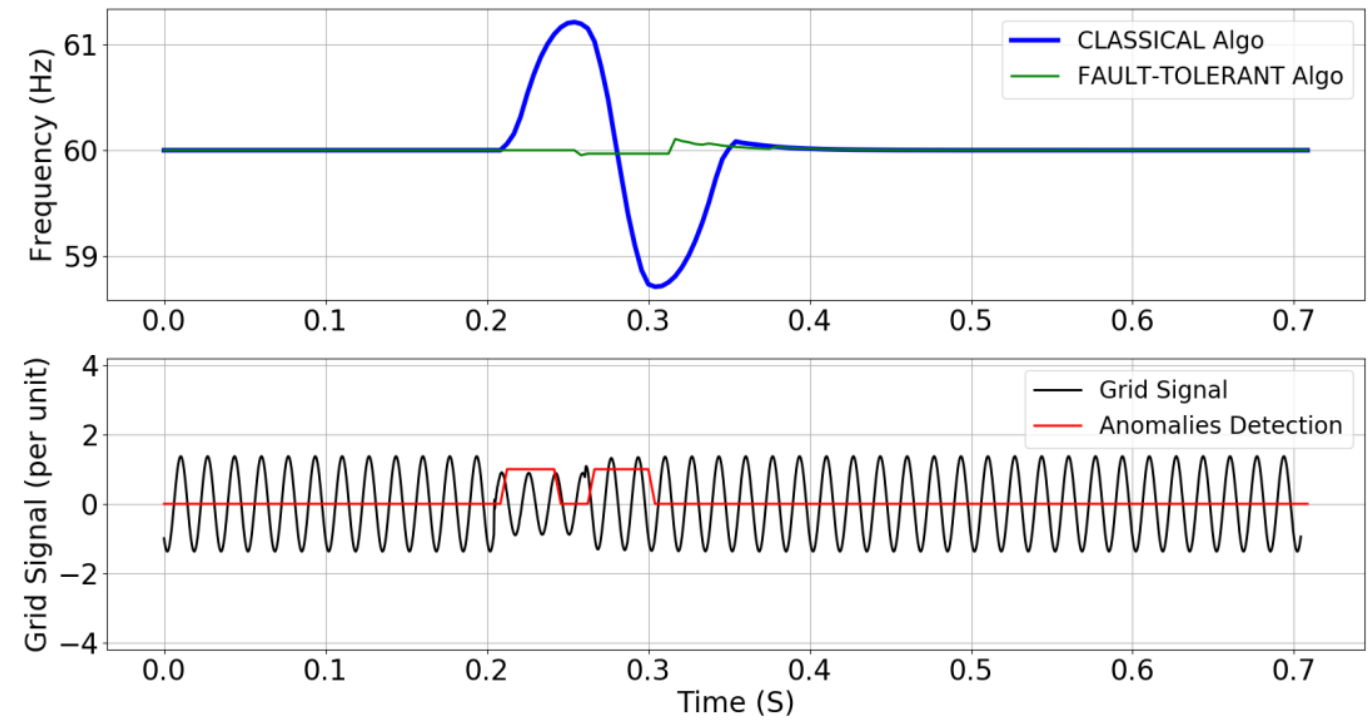

Figure 140 time domain result - case 10
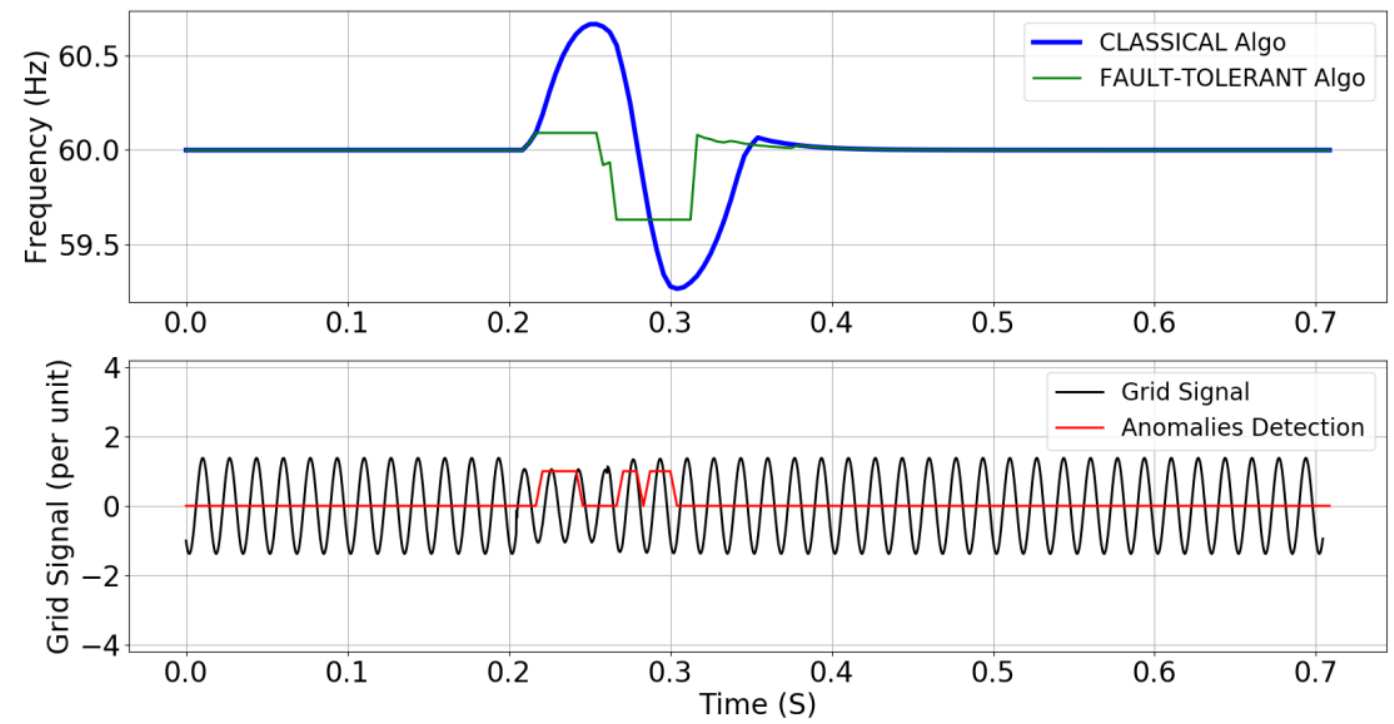

Figure 141 time domain result - case 11 

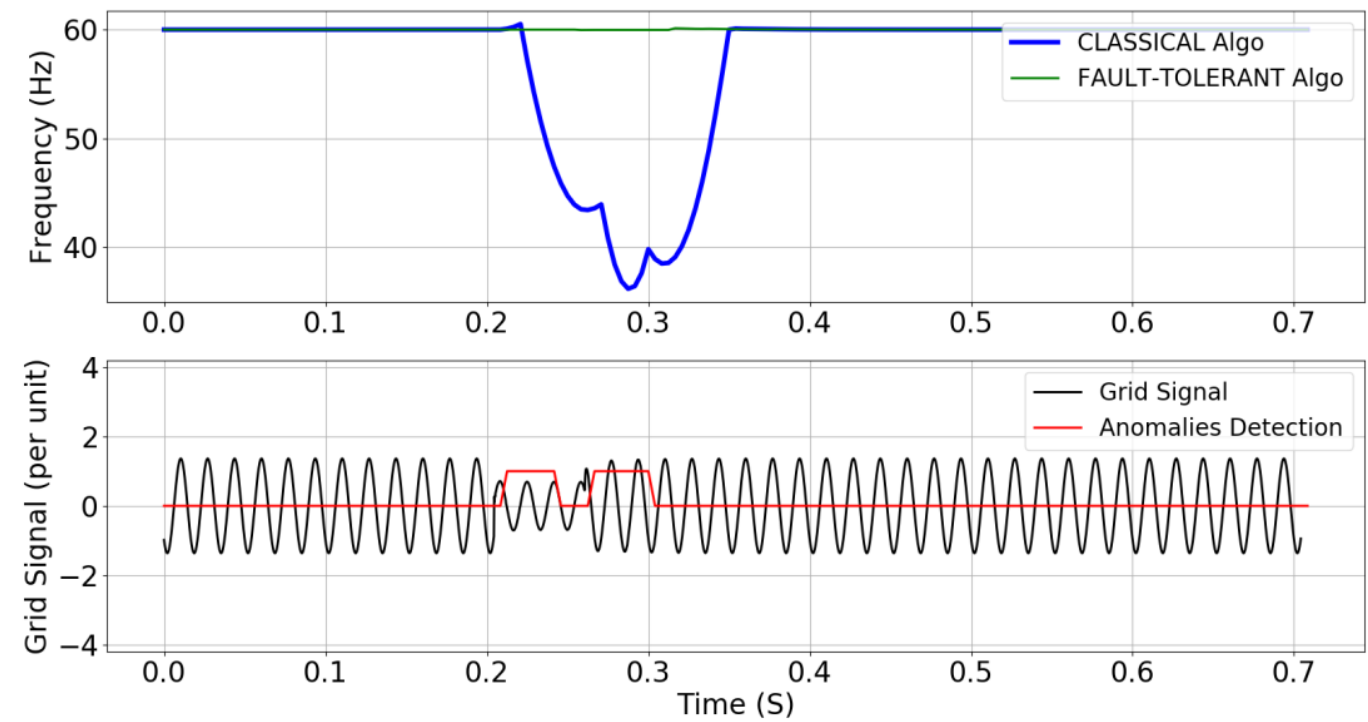

Figure 142 time domain result - case 12
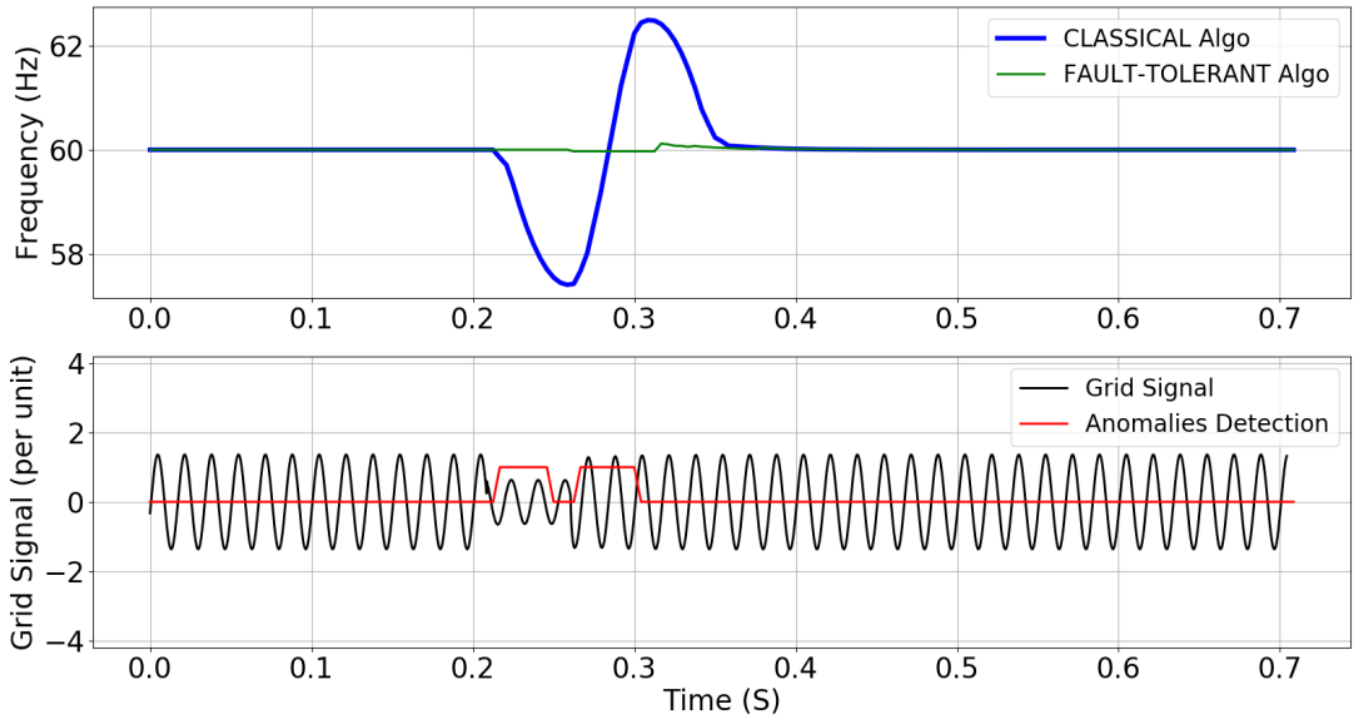

Figure 143 time domain result - case 14 

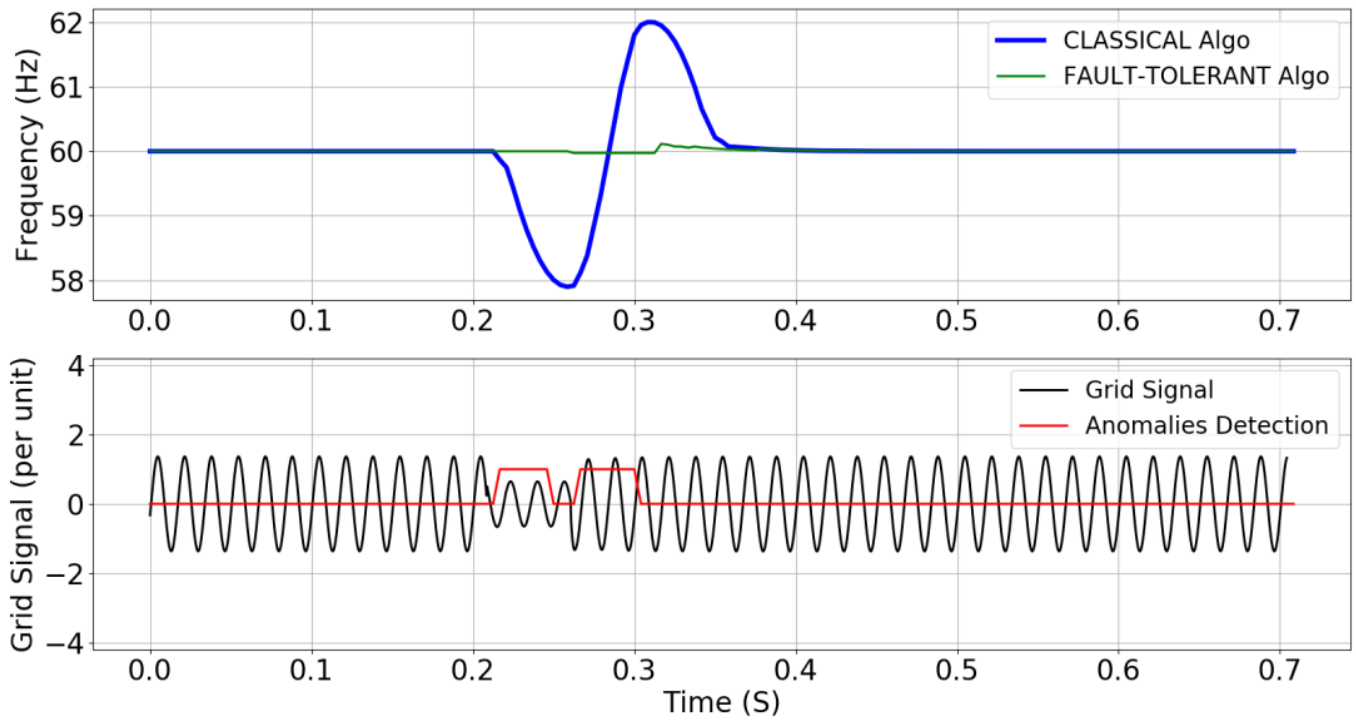

Figure 144 time domain result - case 15
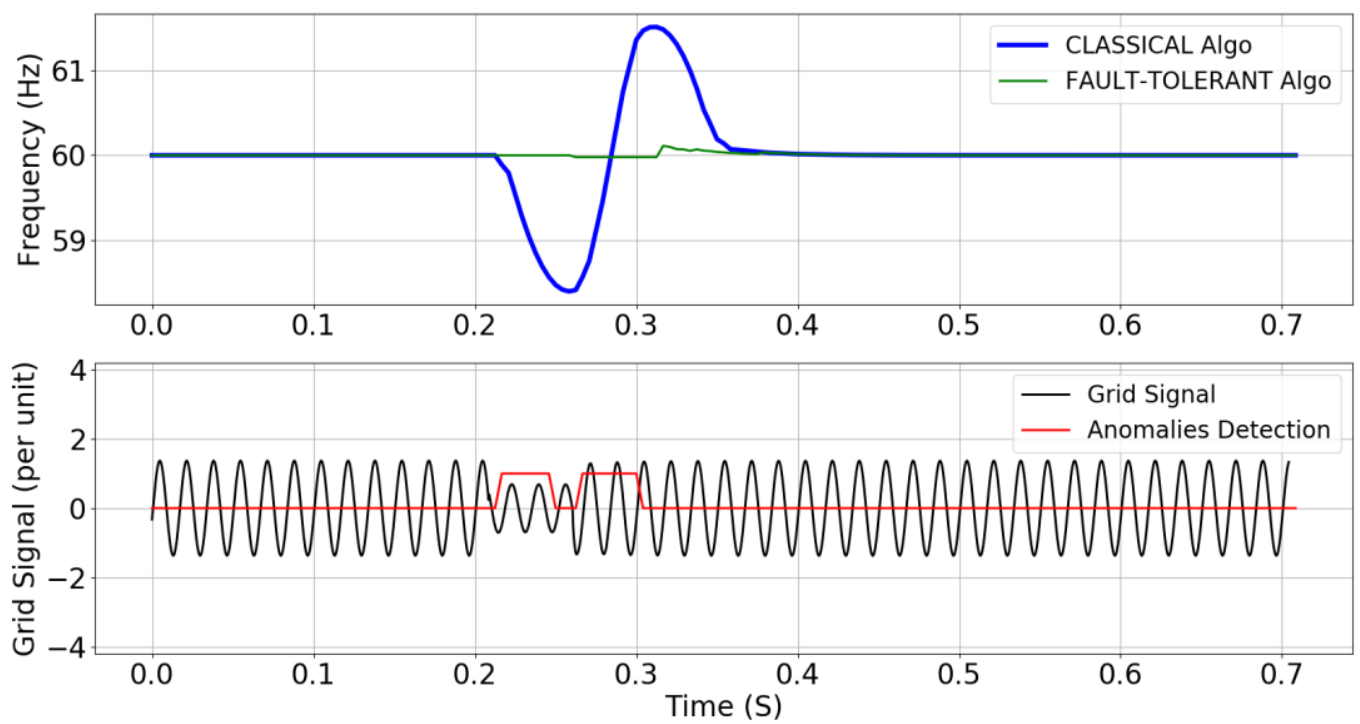

Figure 145 time domain result - case 16 

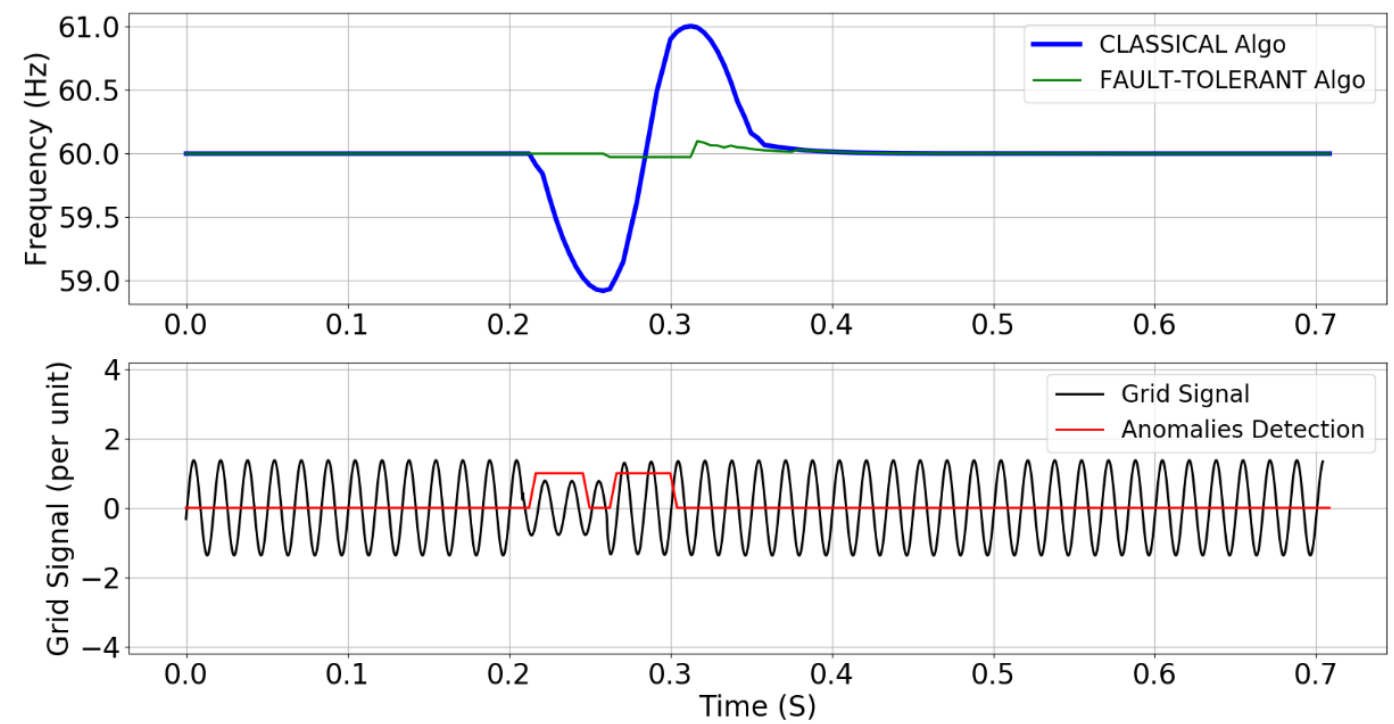

Figure 146 time domain result - case 17
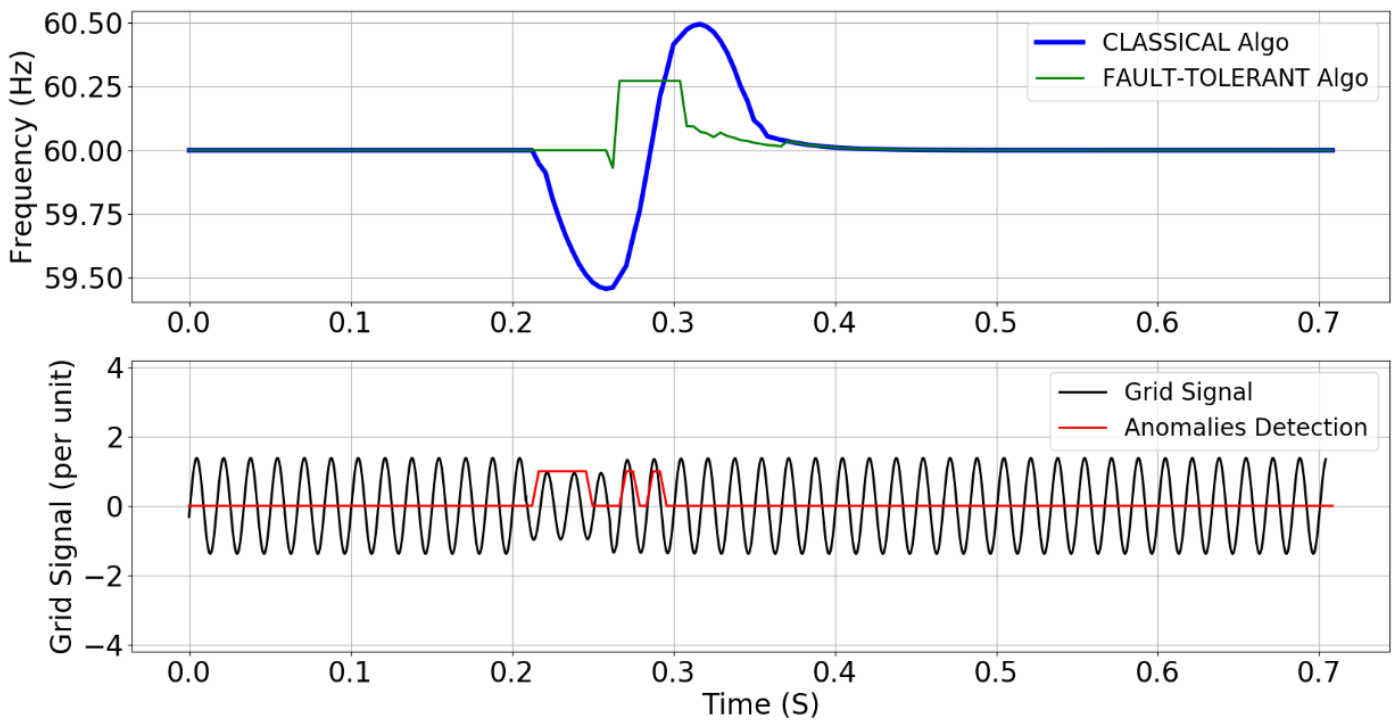

Figure 147 time domain result - case 18 

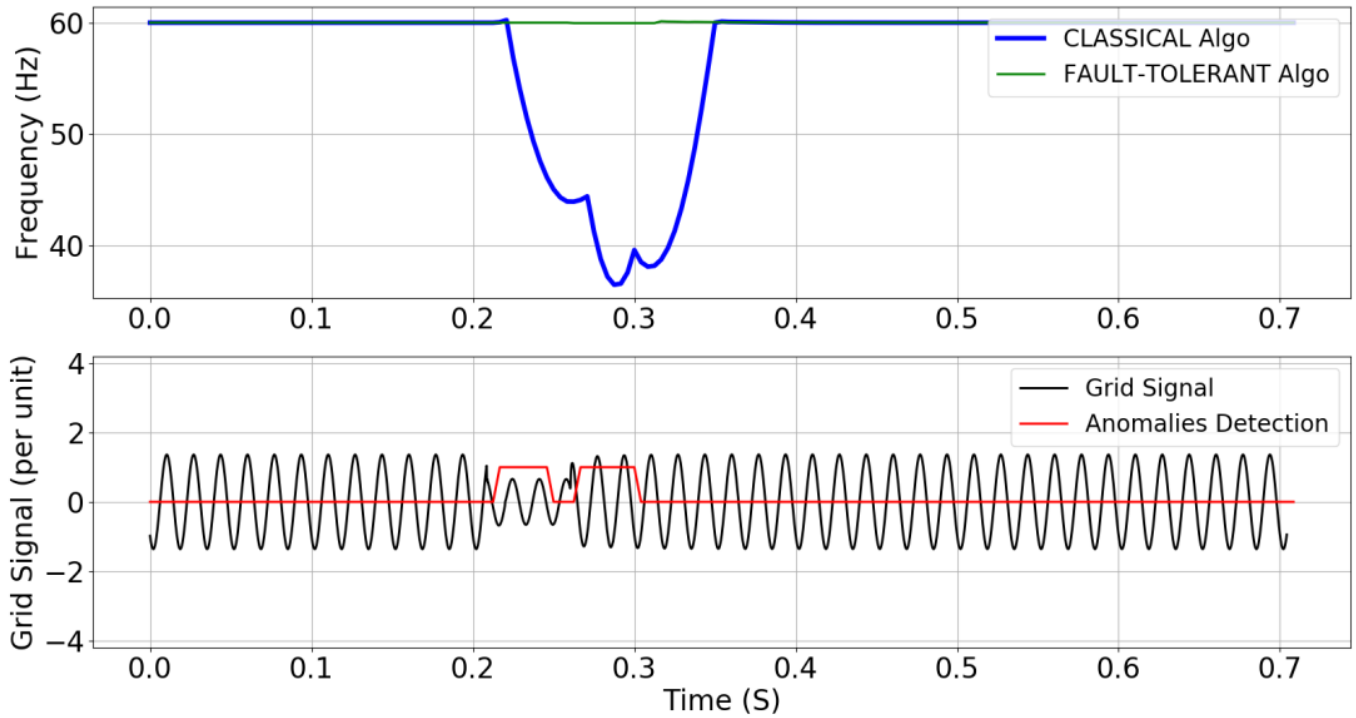

Figure 148 time domain result - case 19
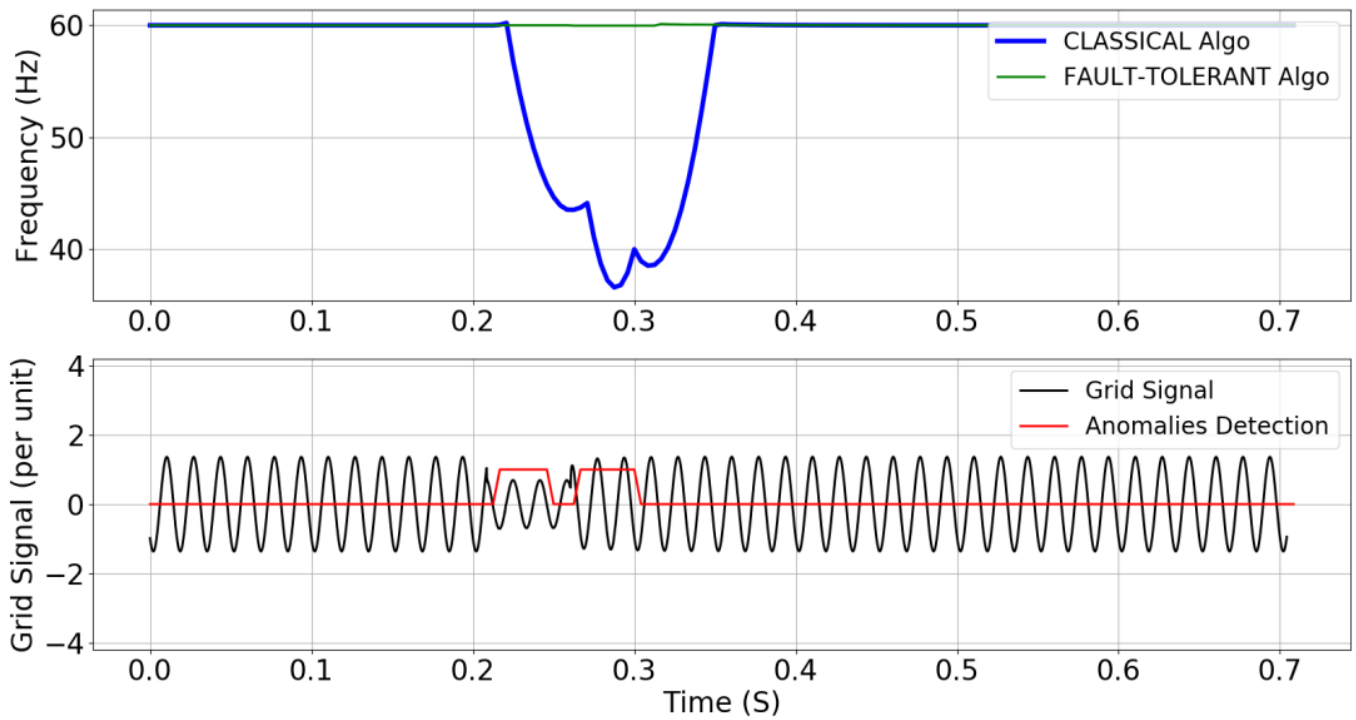

Figure 149 time domain result - case 20 

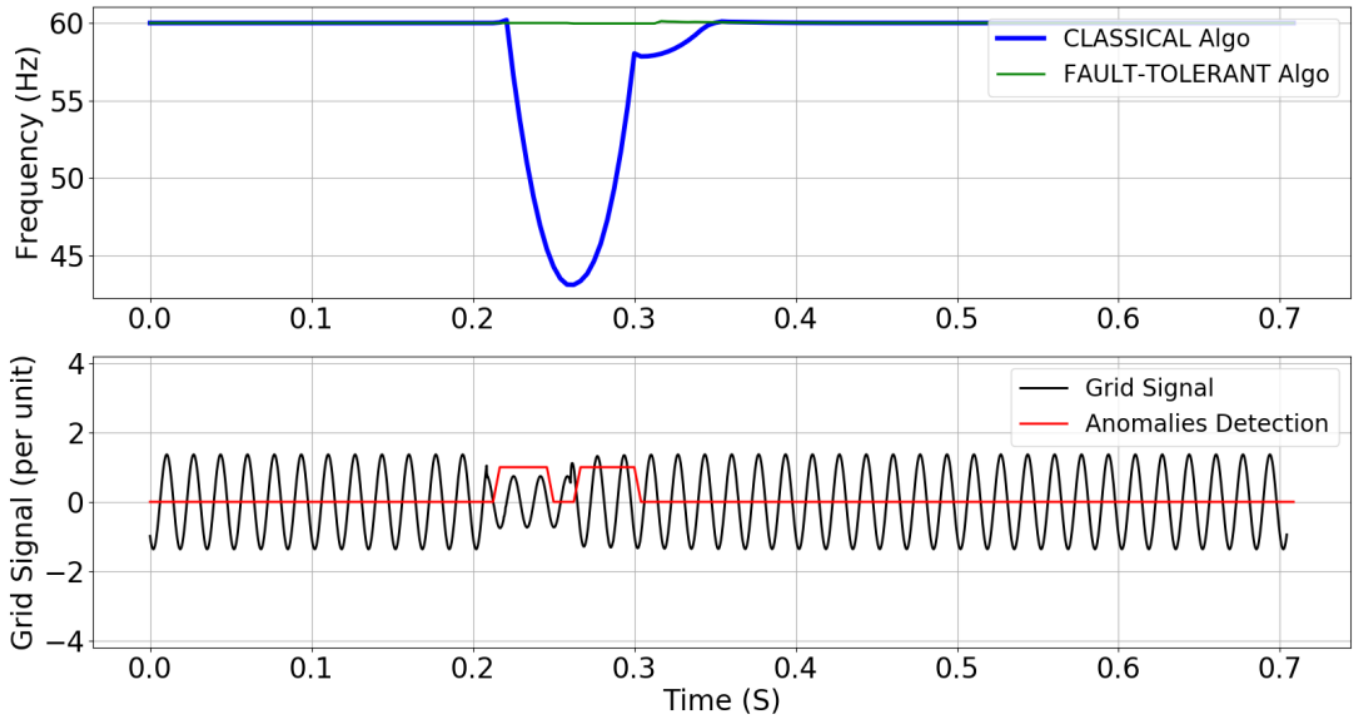

Figure 150 time domain result - case 21
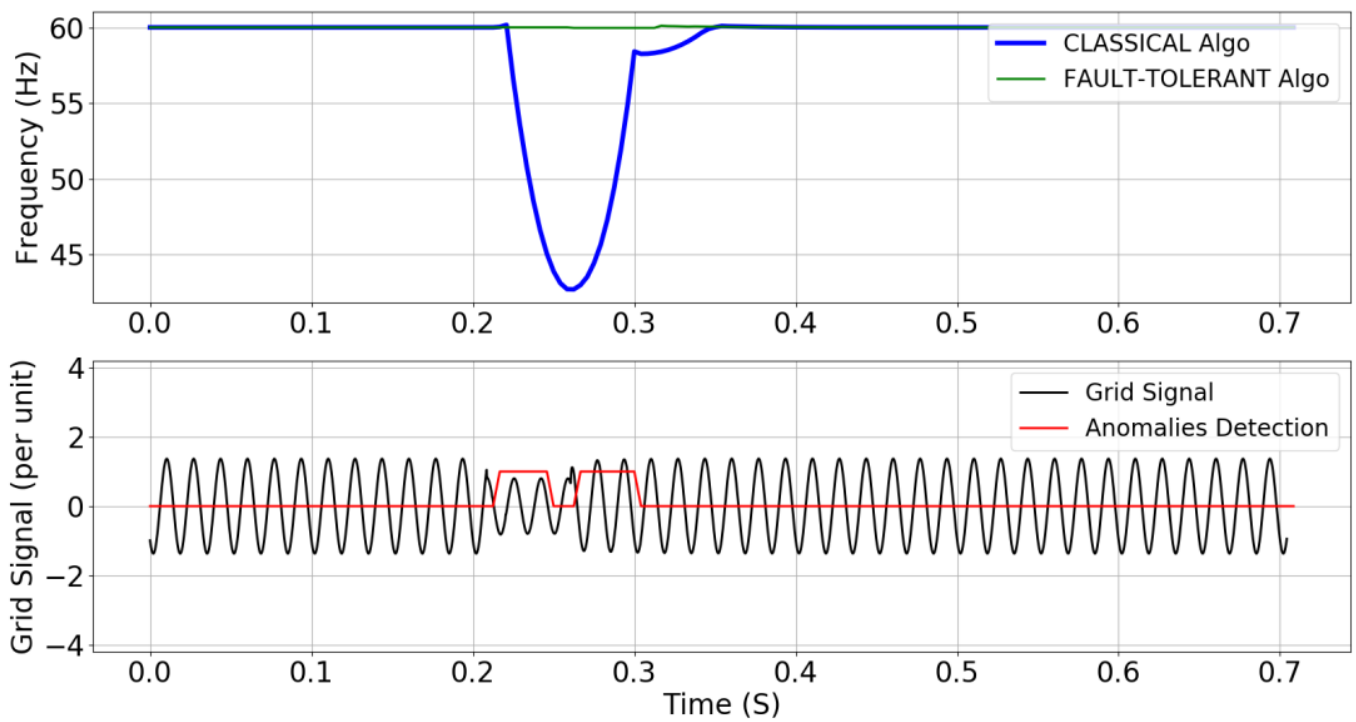

Figure 151 time domain result - case 22 

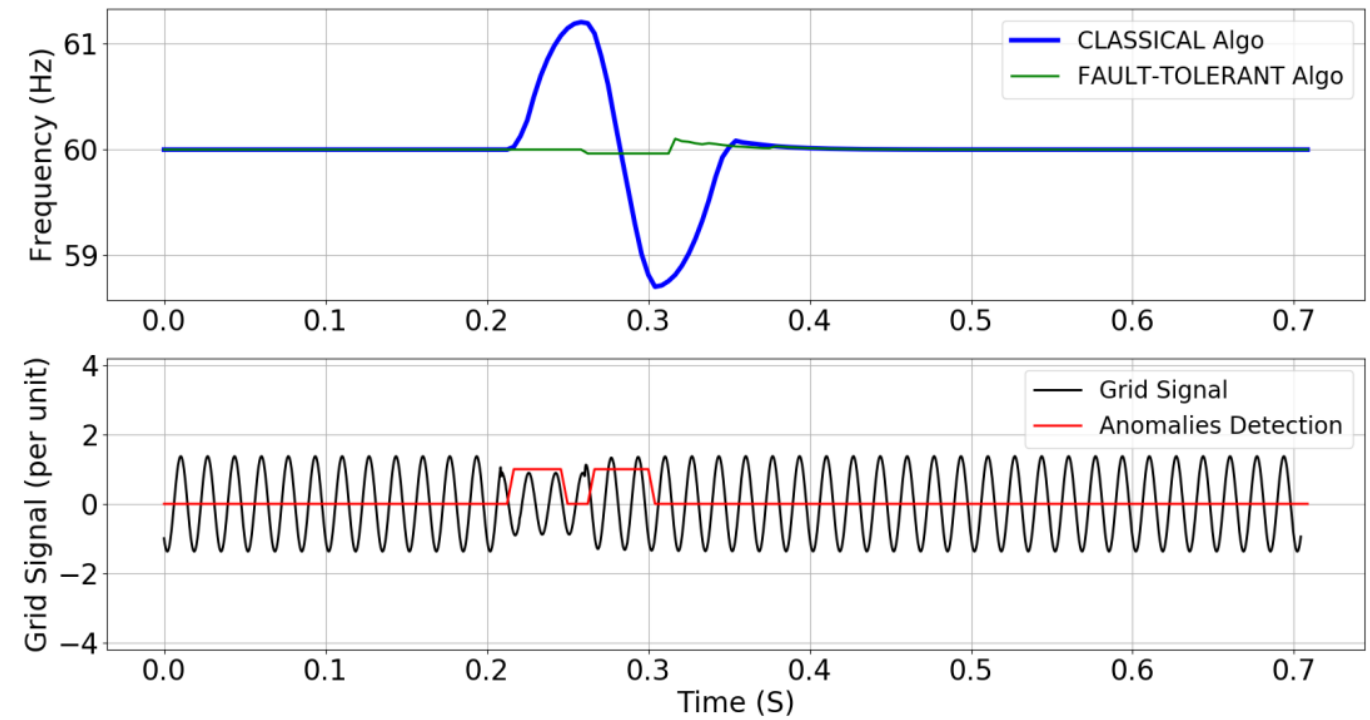

Figure 152 time domain result - case 23
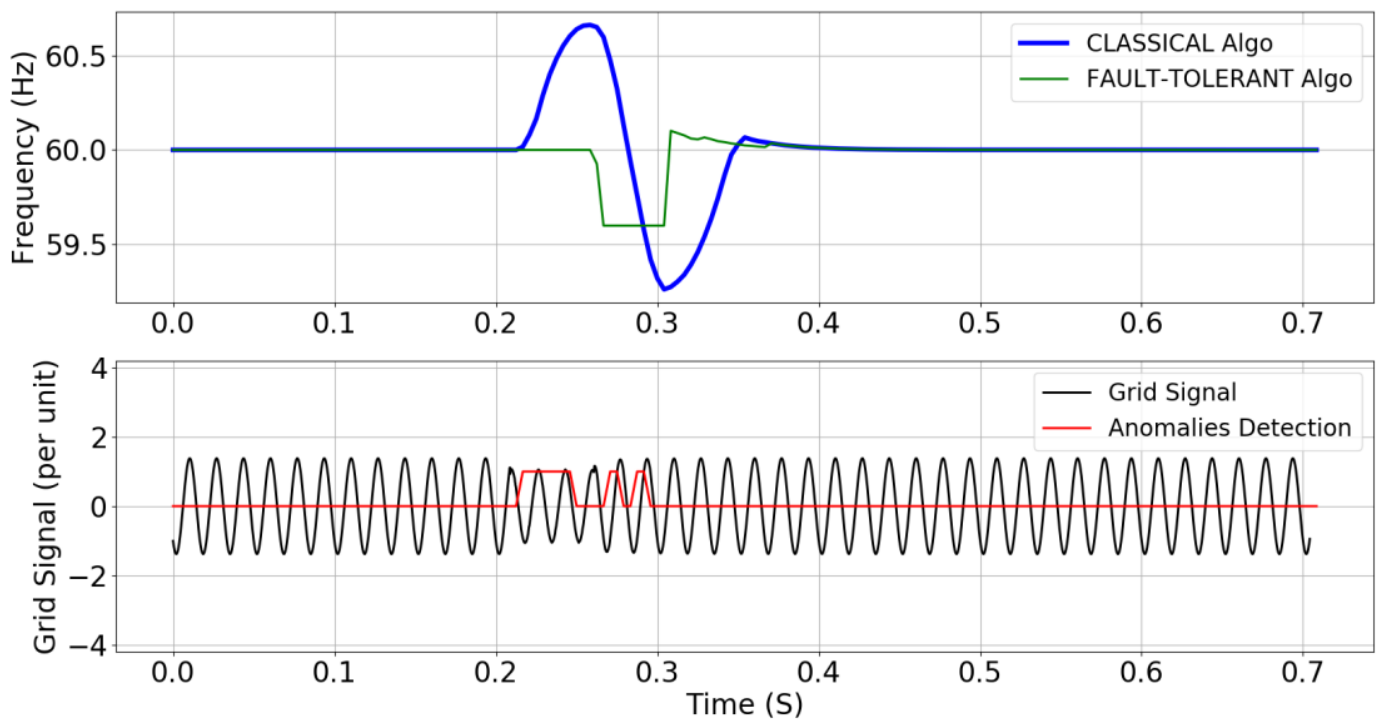

Figure 153 time domain result - case 24 

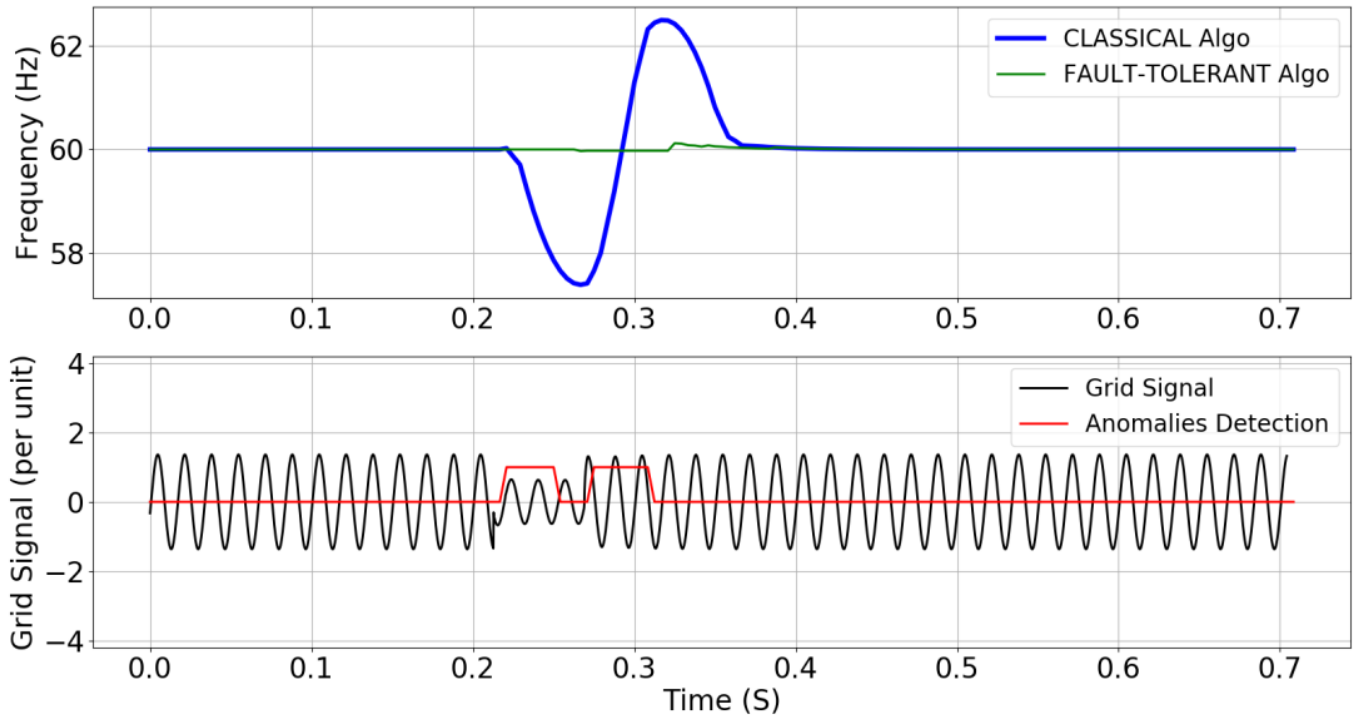

Figure 154 time domain result - case 26
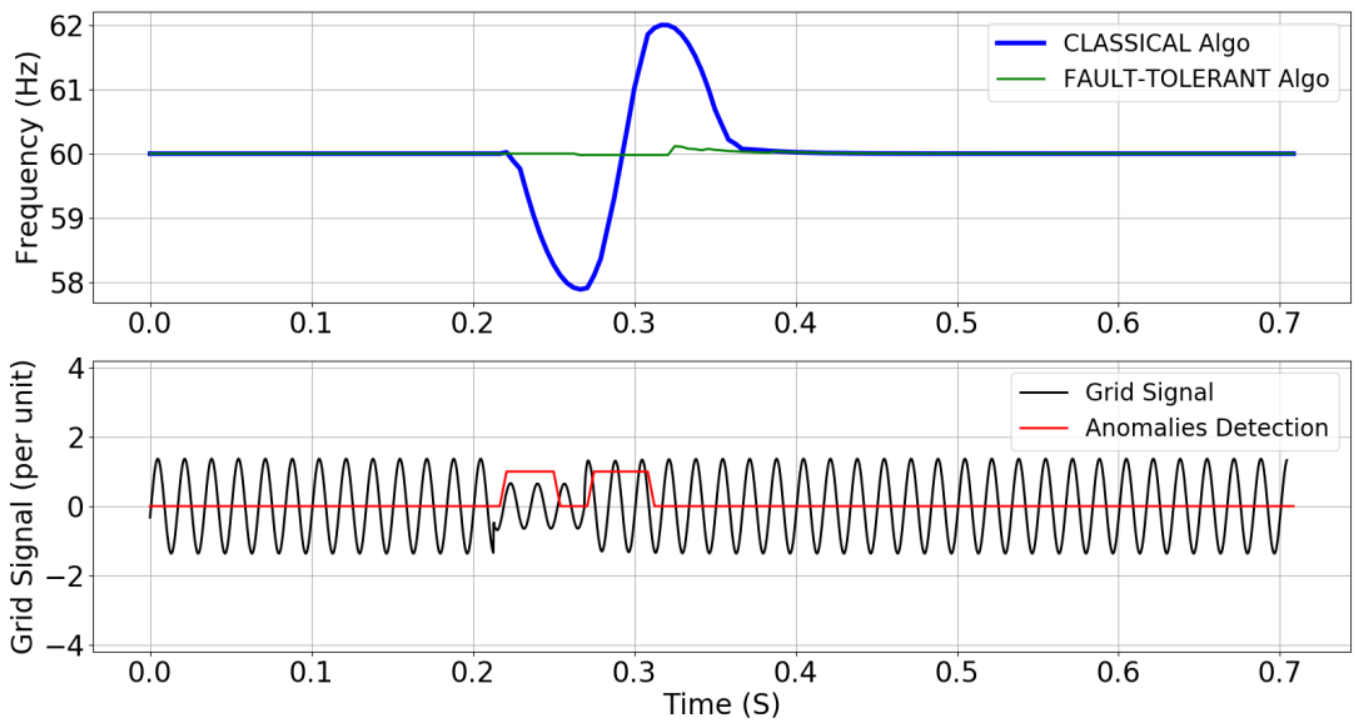

Figure 155 time domain result - case 27 

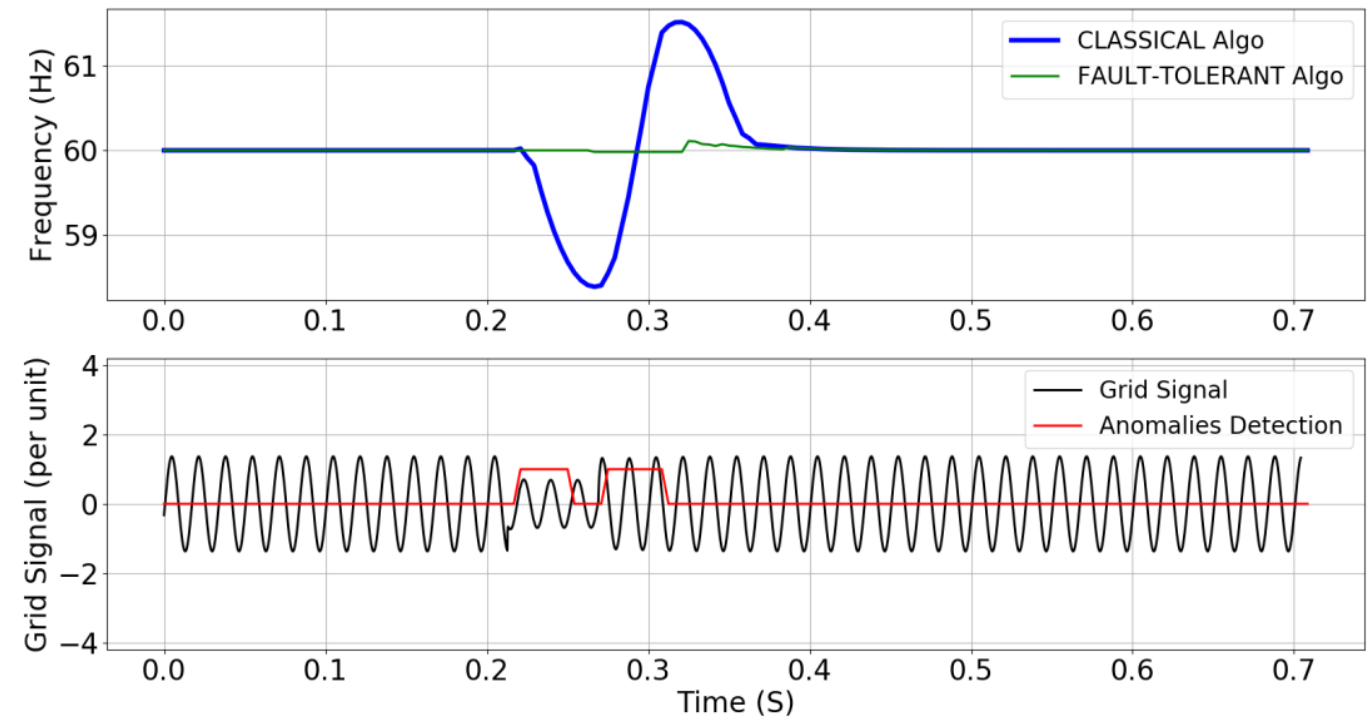

Figure 156 time domain result - case 28
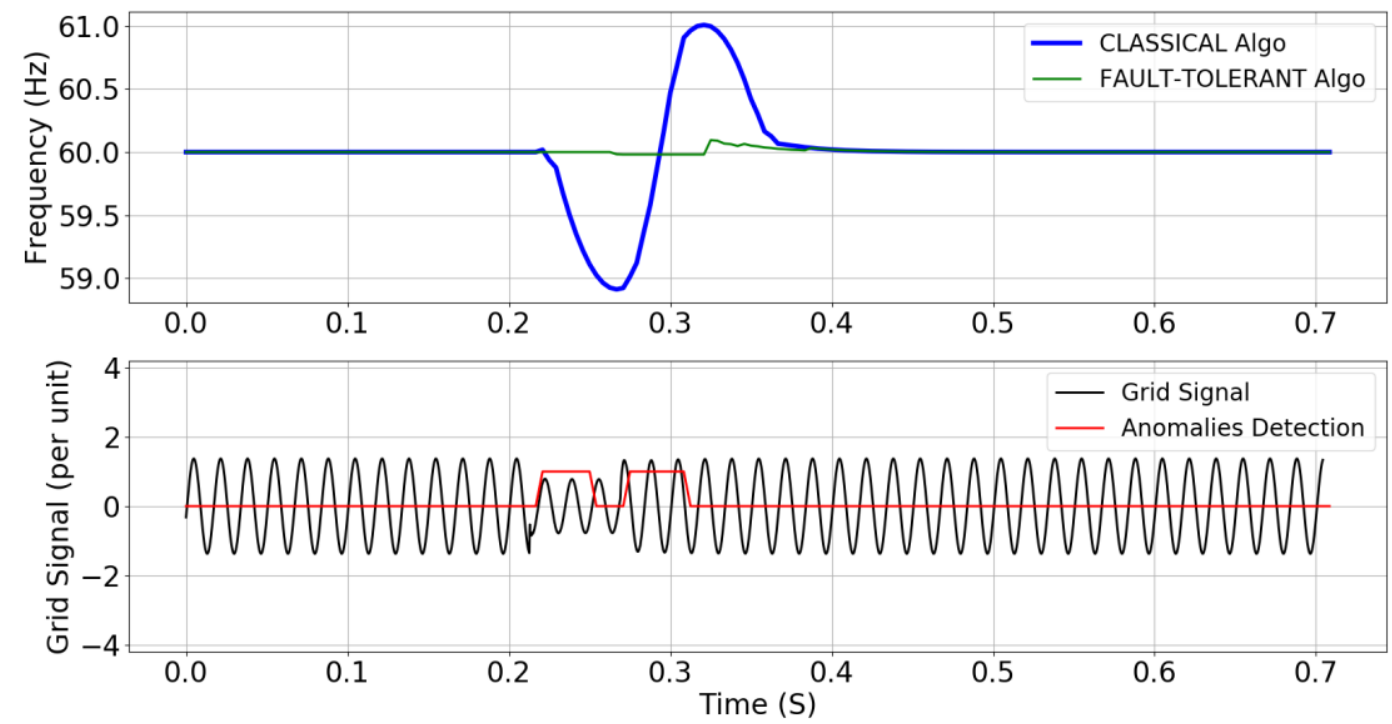

Figure 157 time domain result - case 29 

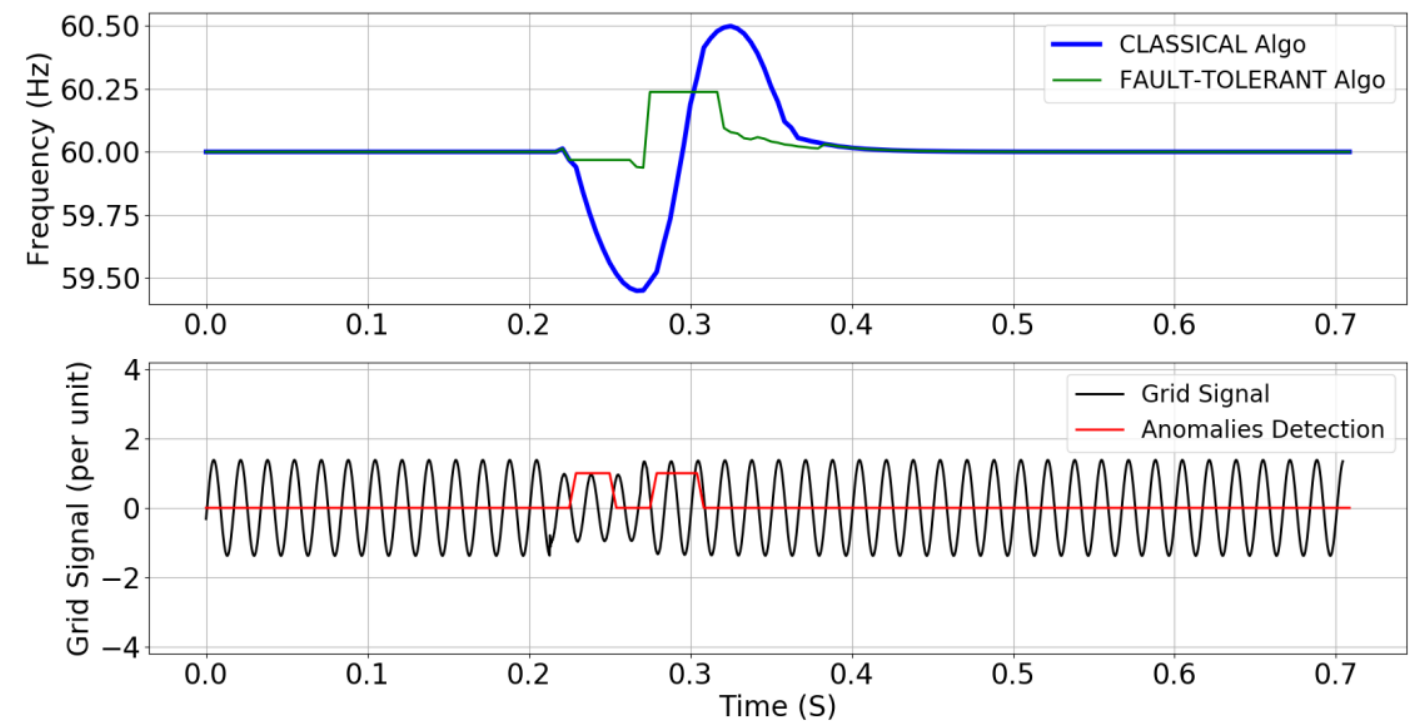

Figure 158 time domain result - case 30
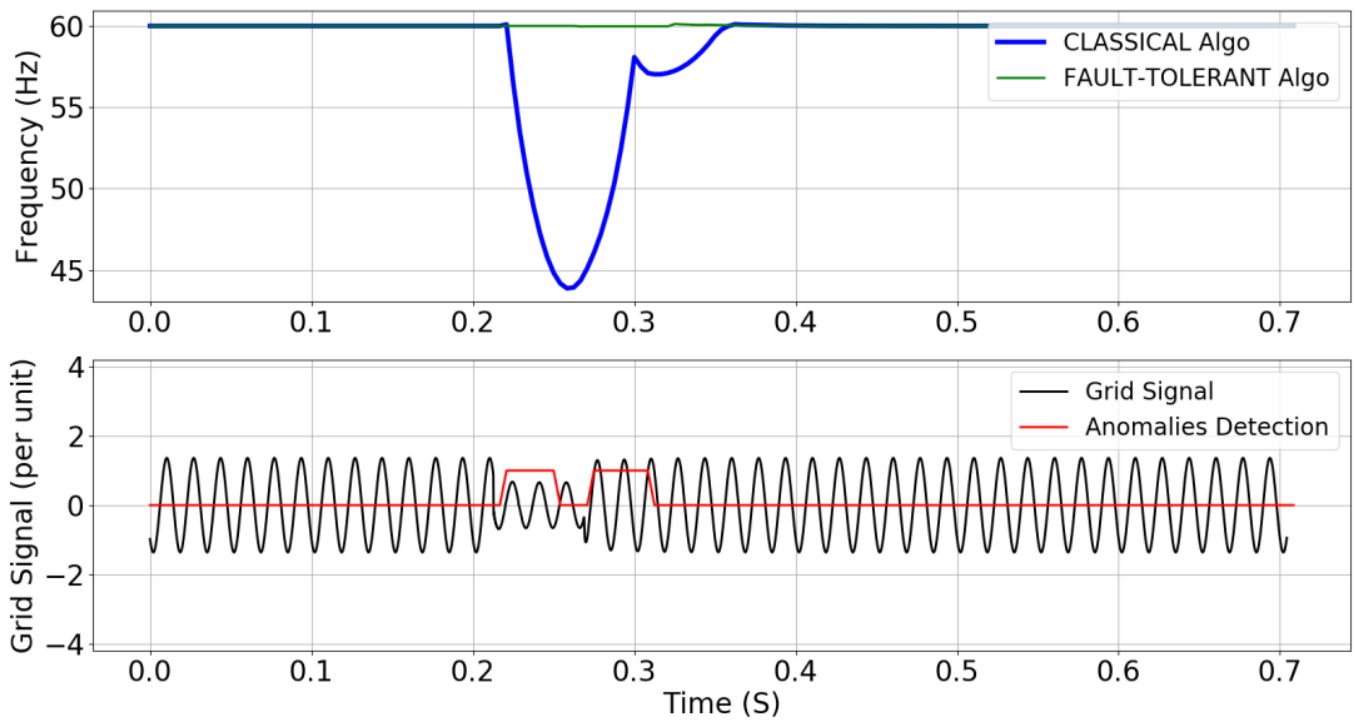

Figure 159 time domain result - case 31 

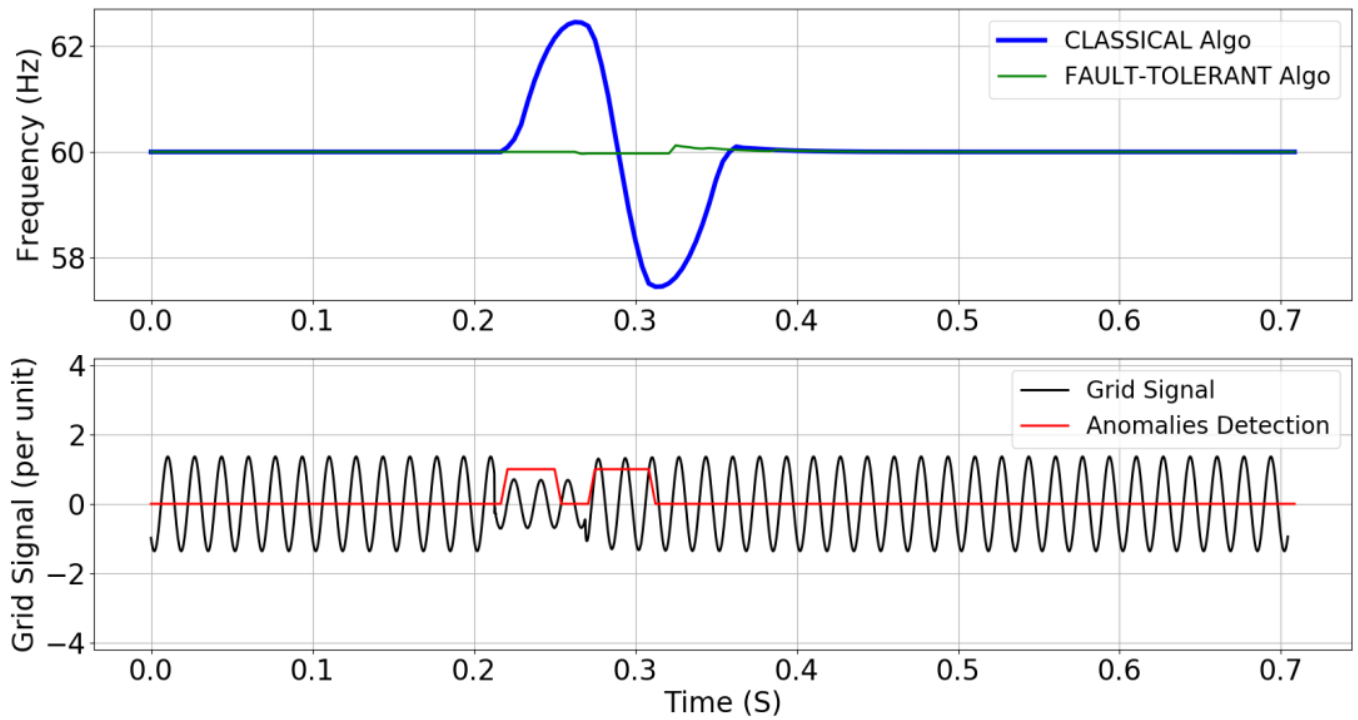

Figure 160 time domain result - case 32
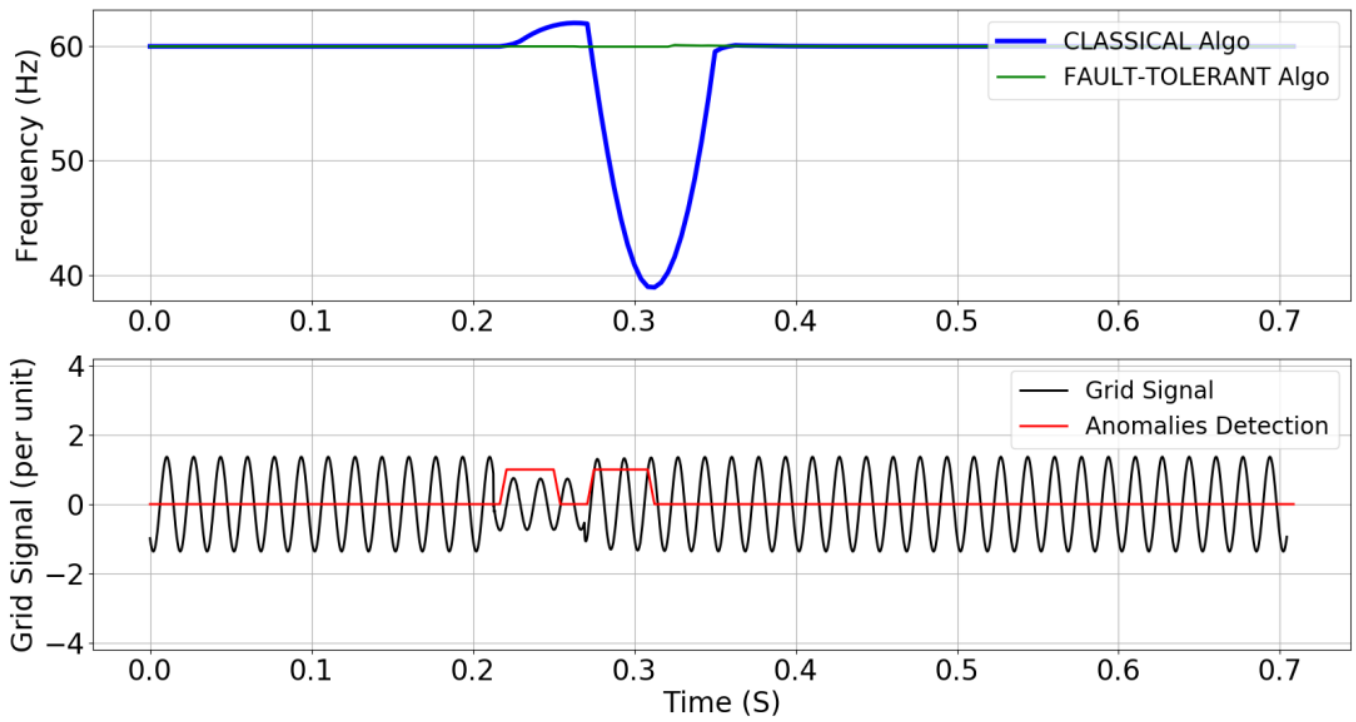

Figure 161 time domain result - case 33 

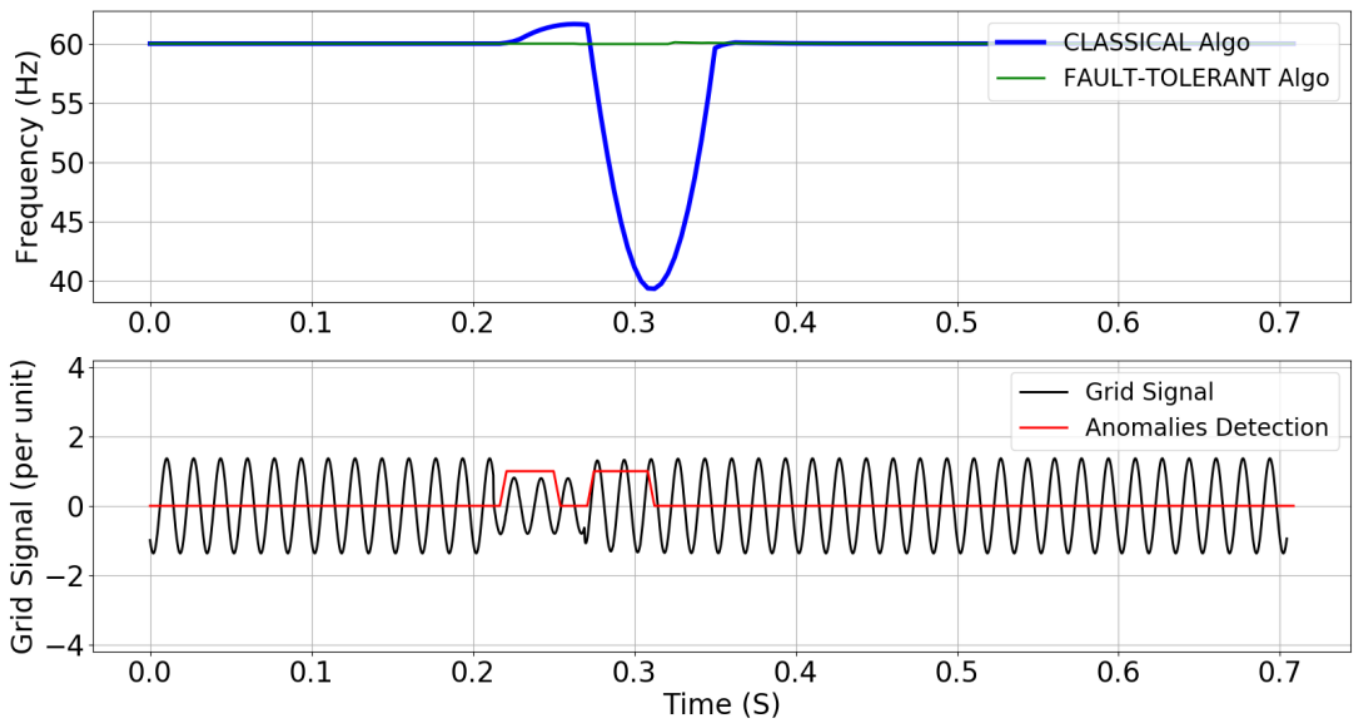

Figure 162 time domain result - case 34
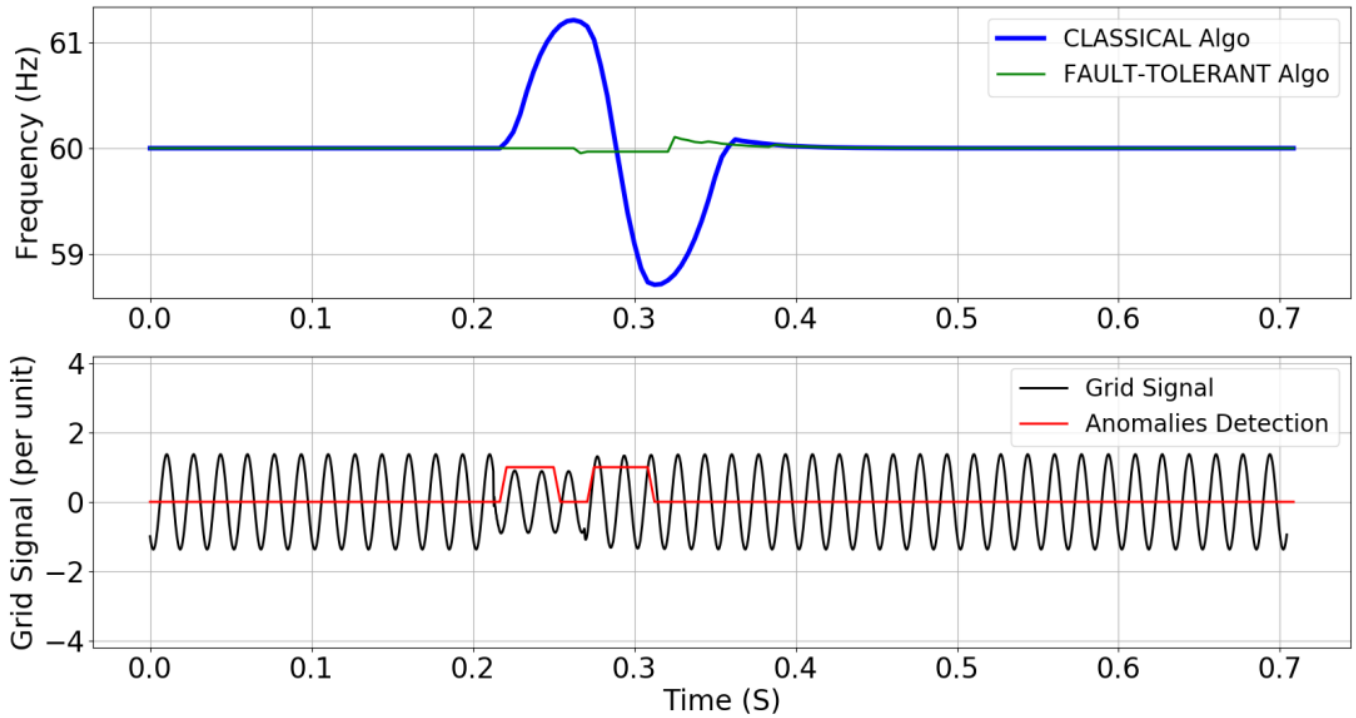

Figure 163 time domain result - case 35 

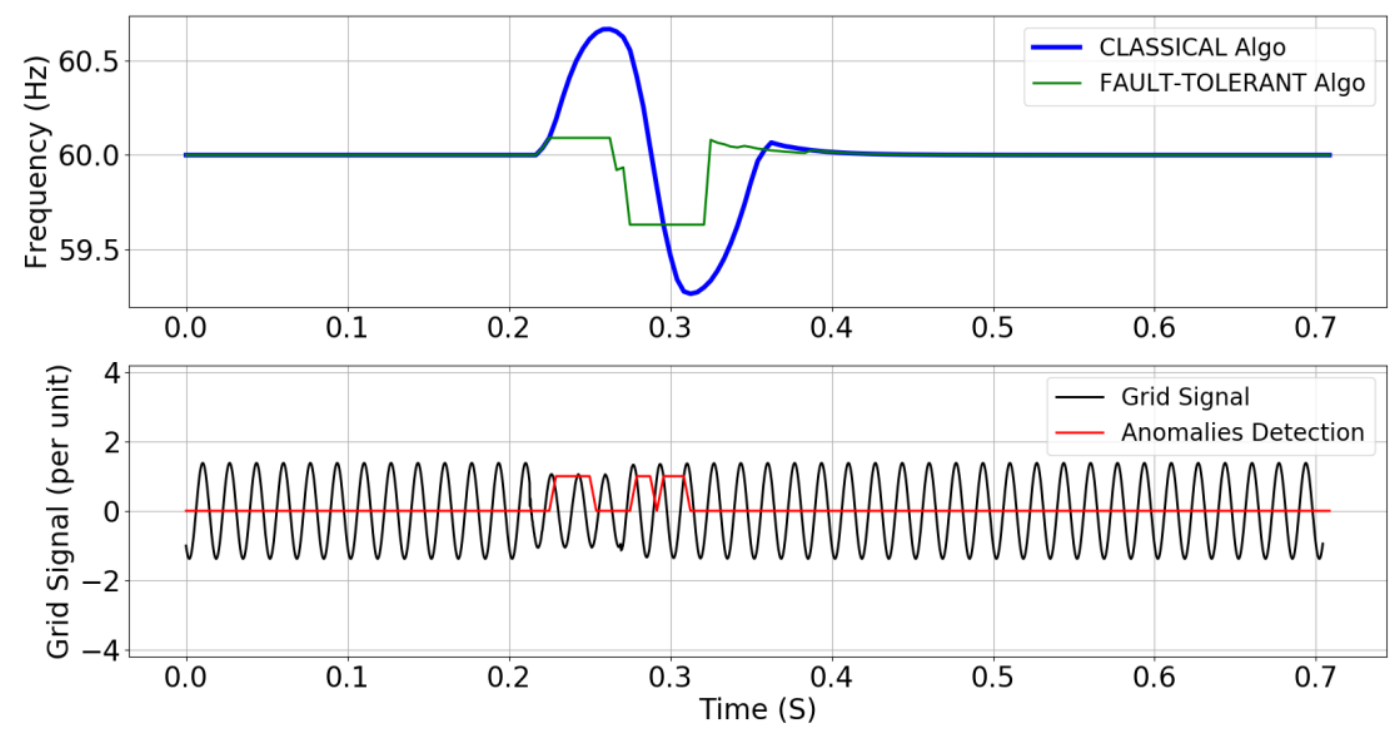

Figure 164 time domain result - case 36
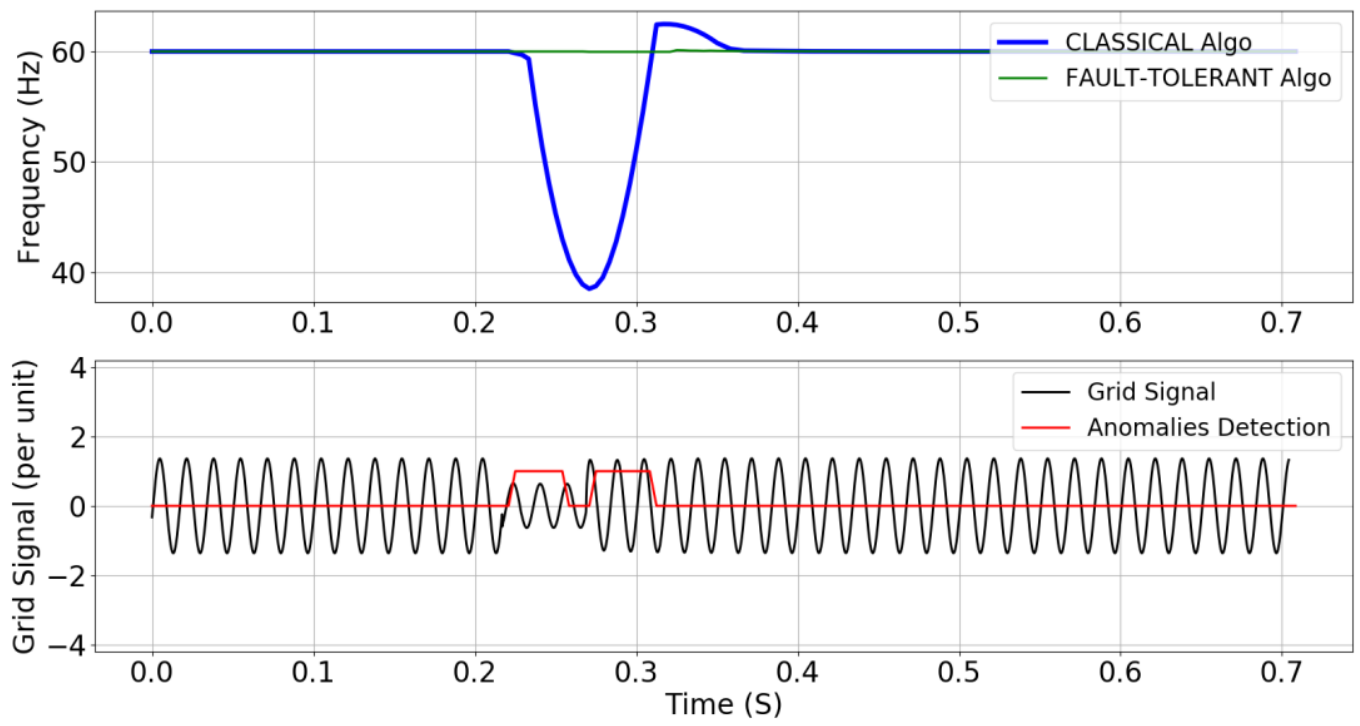

Figure 165 time domain result - case 38 

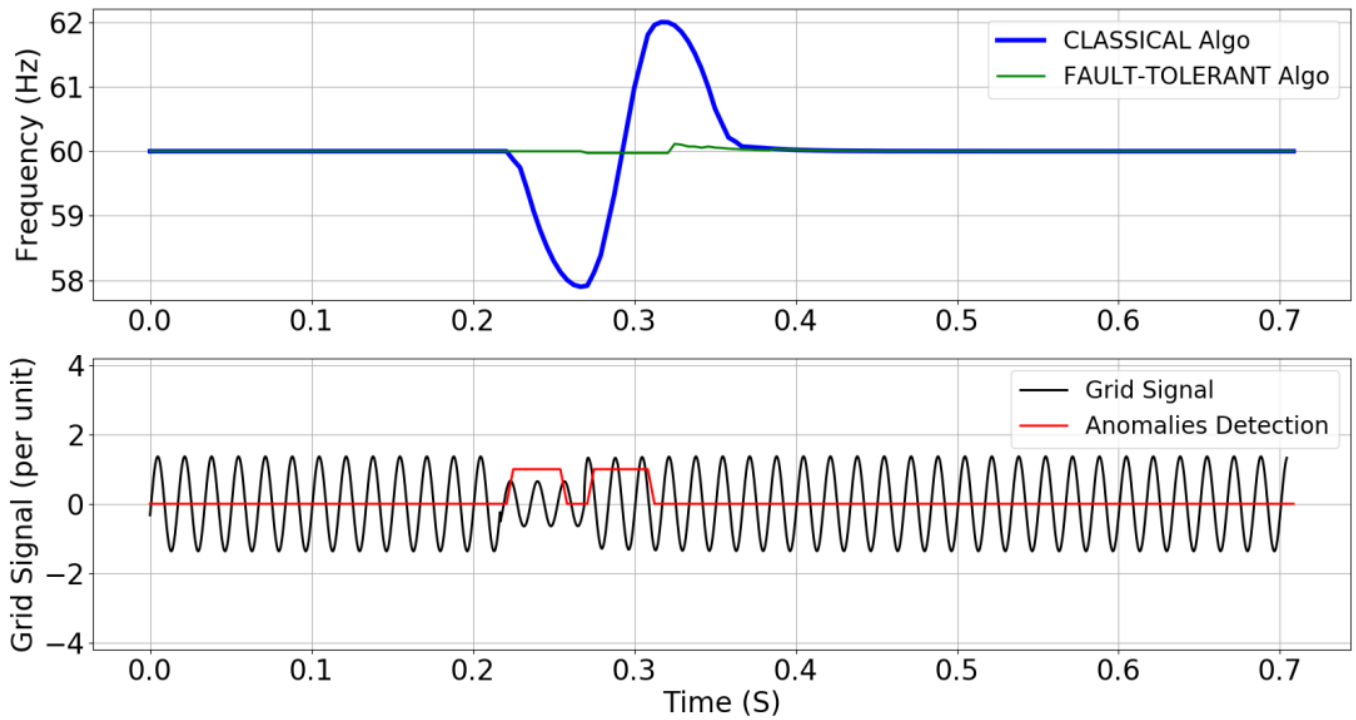

Figure 166 time domain result - case 39
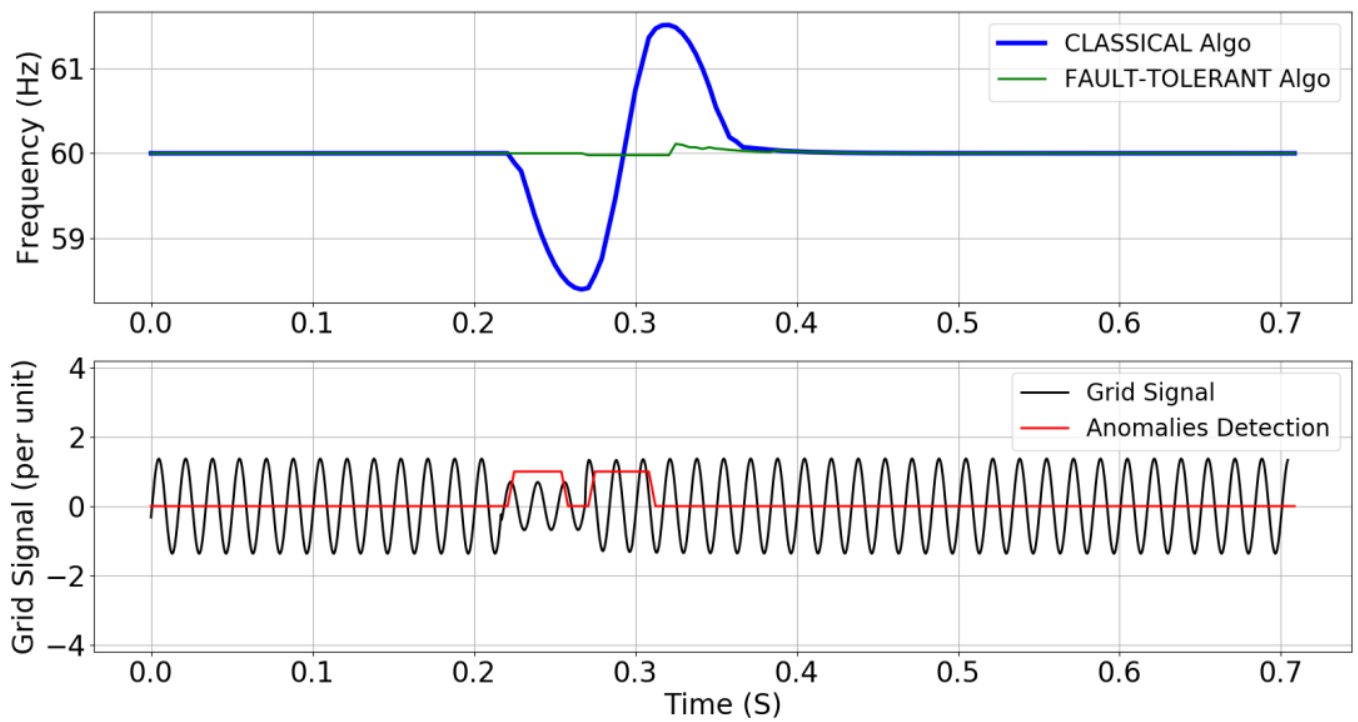

Figure 167 time domain result - case 40 

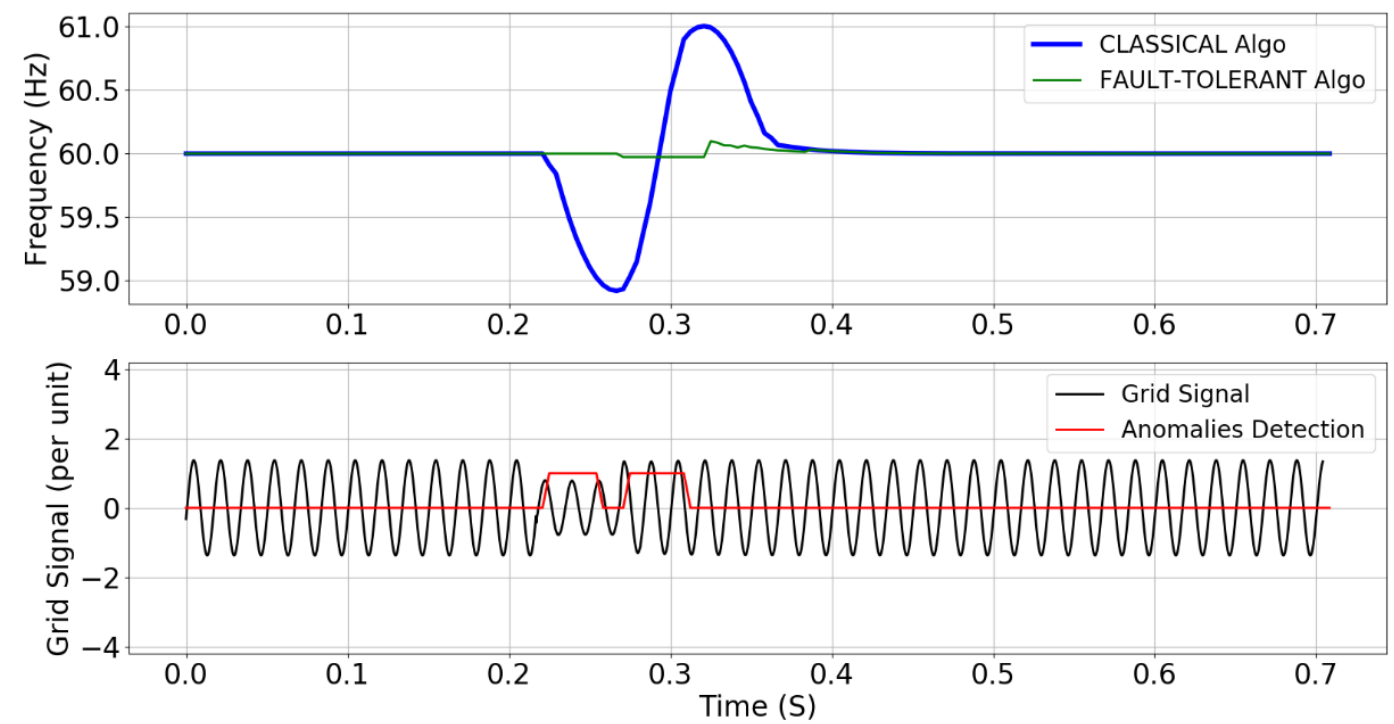

Figure 168 time domain result - case 41
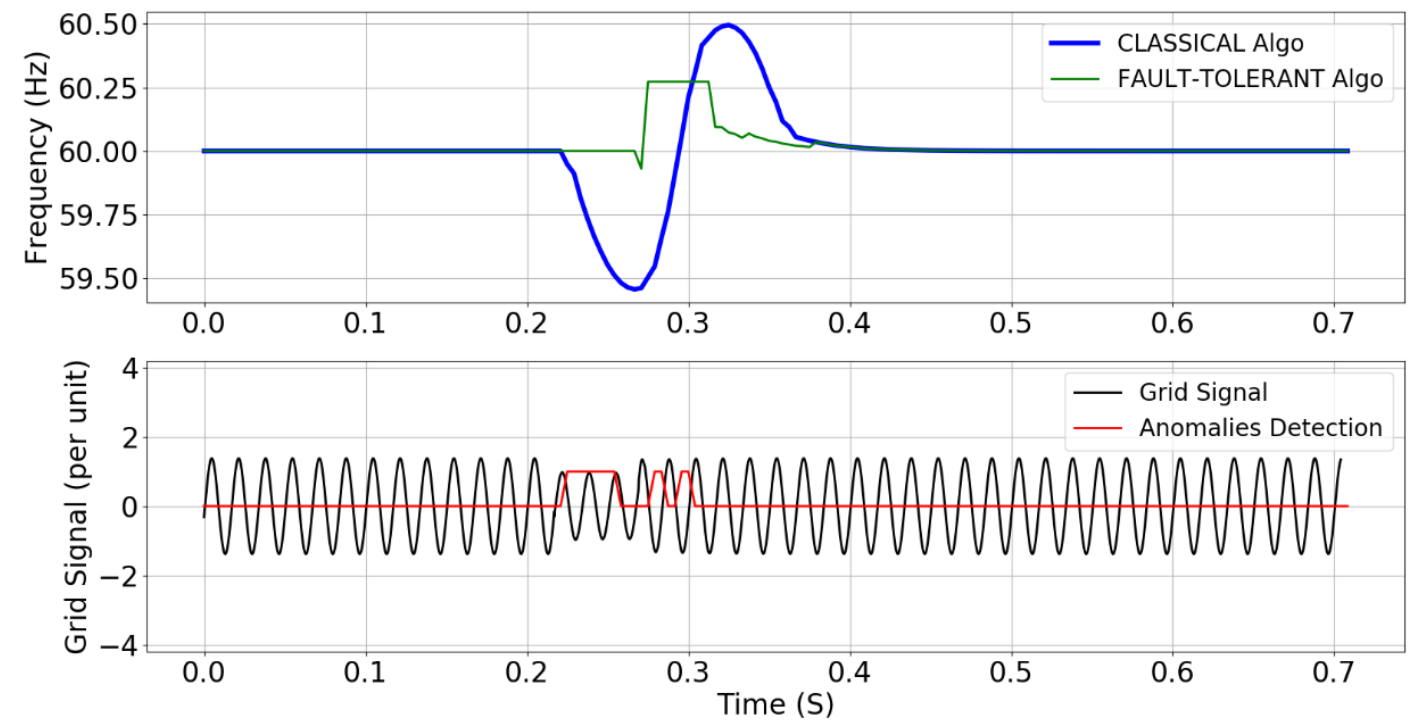

Figure 169 time domain result - case 42 

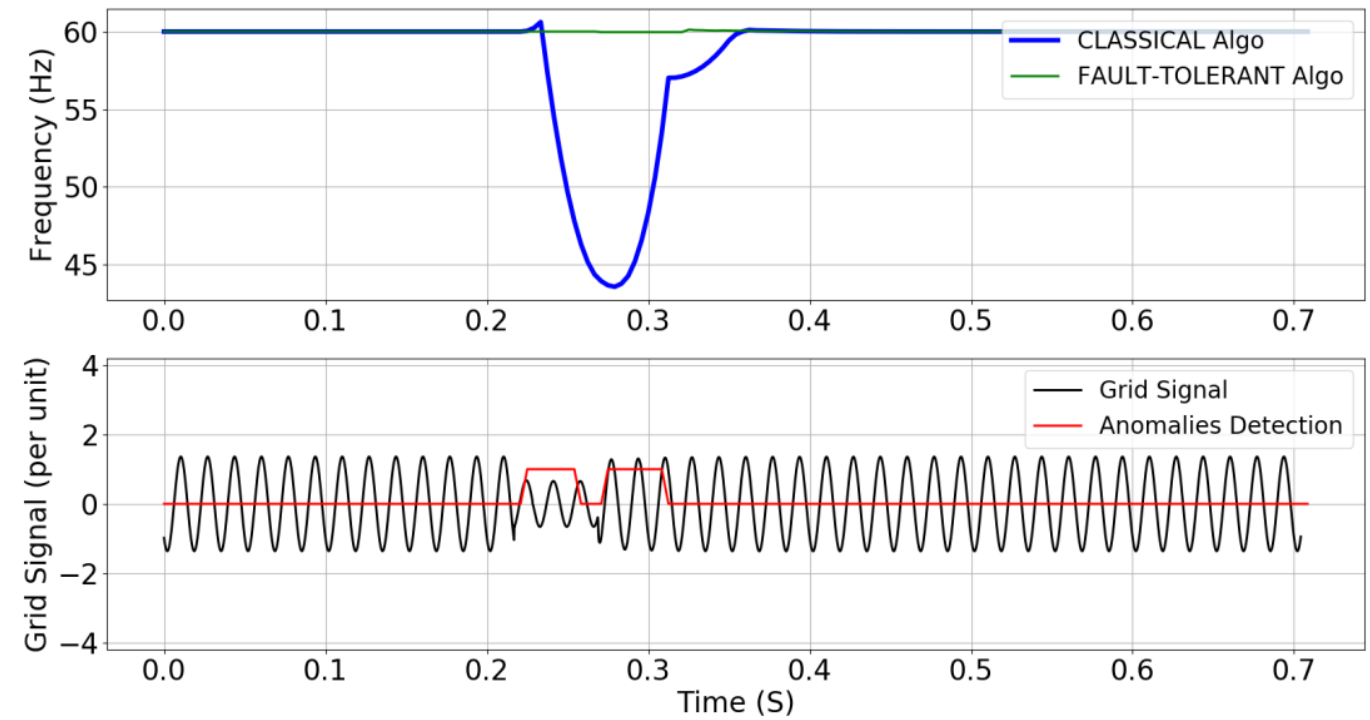

Figure 170 time domain result - case 43
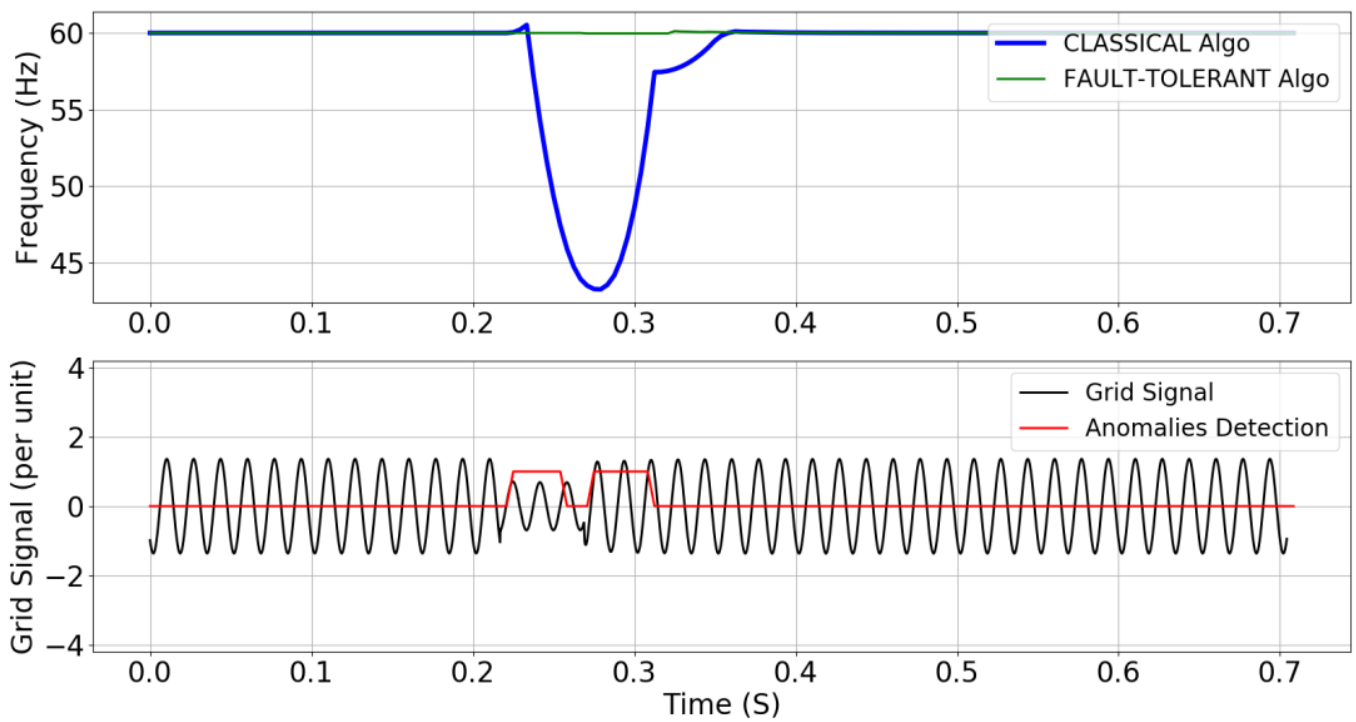

Figure 171 time domain result - case 44 

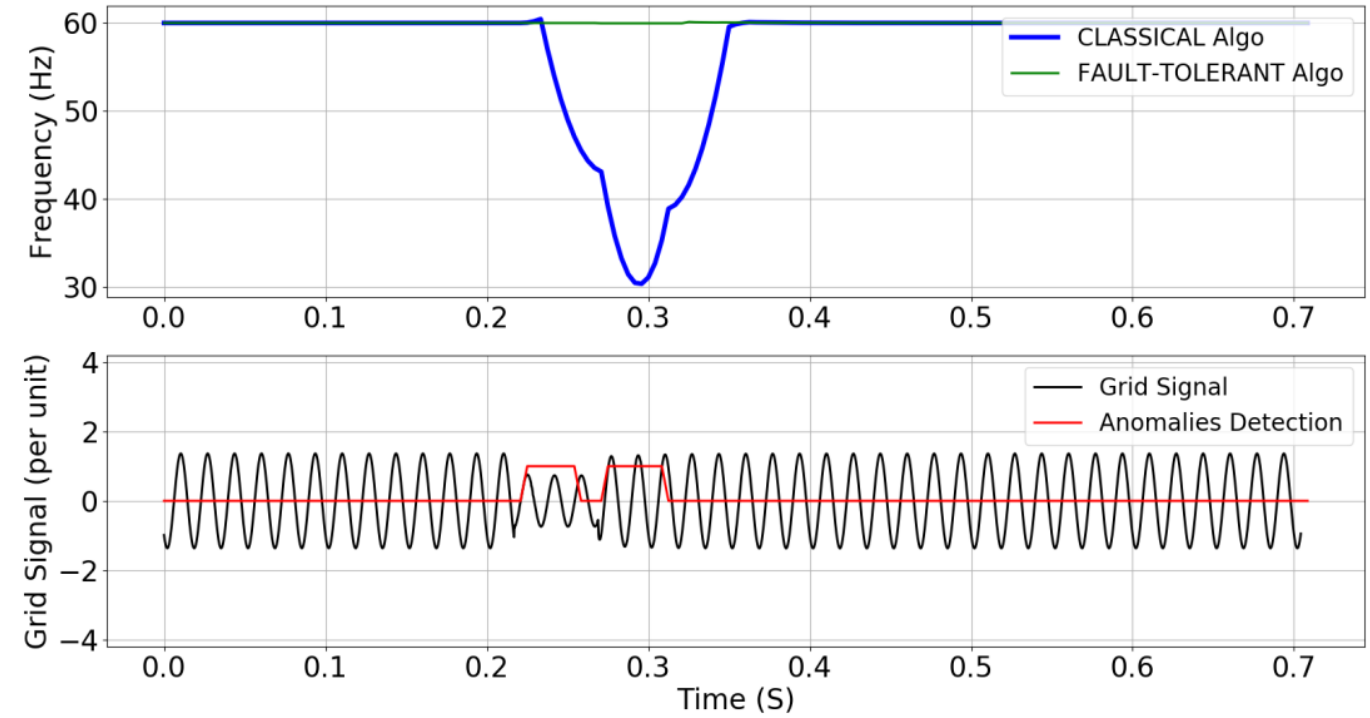

Figure 172 time domain result - case 45
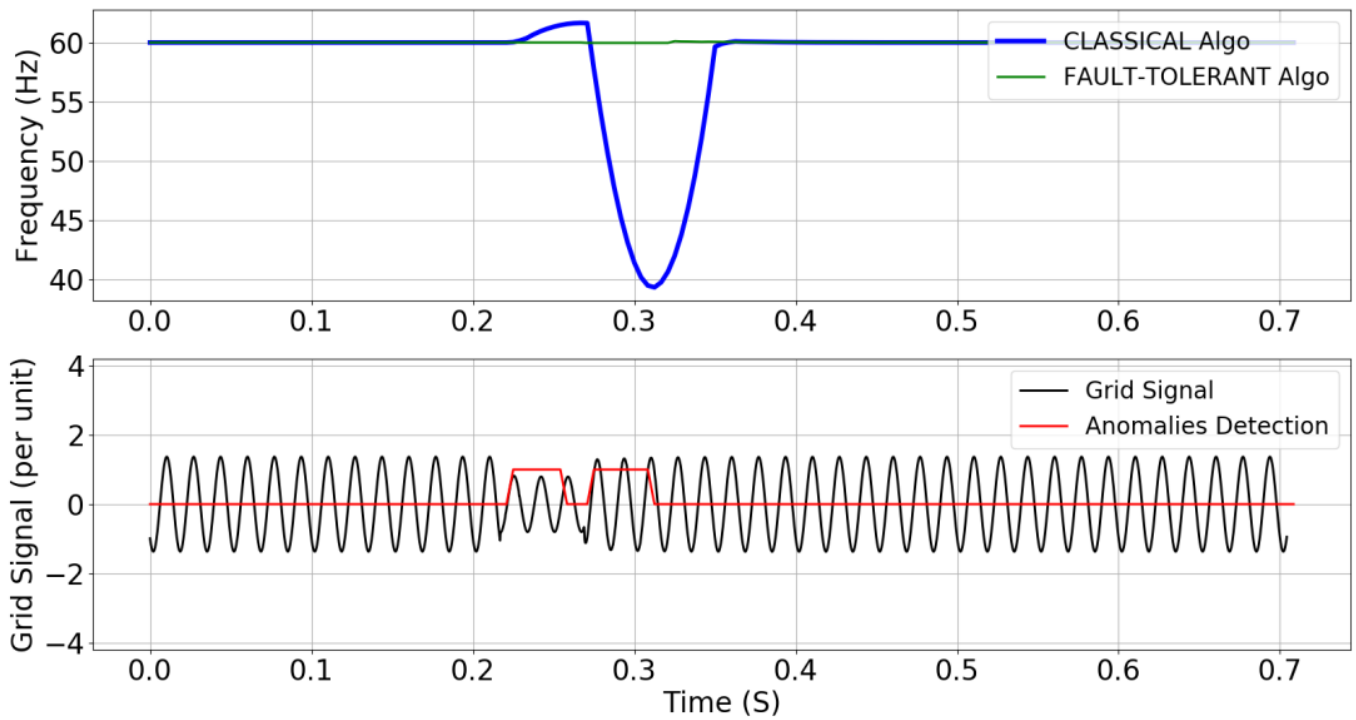

Figure 173 time domain result - case 46 

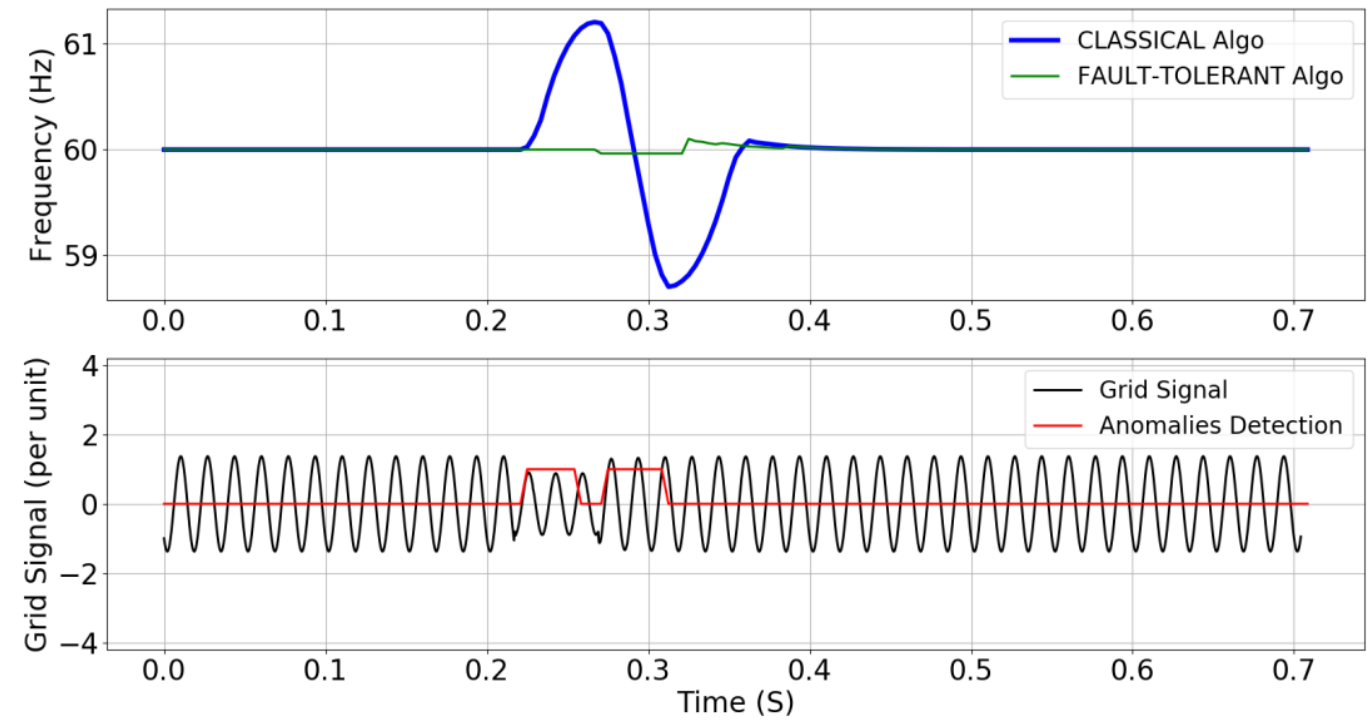

Figure 174 time domain result - case 47
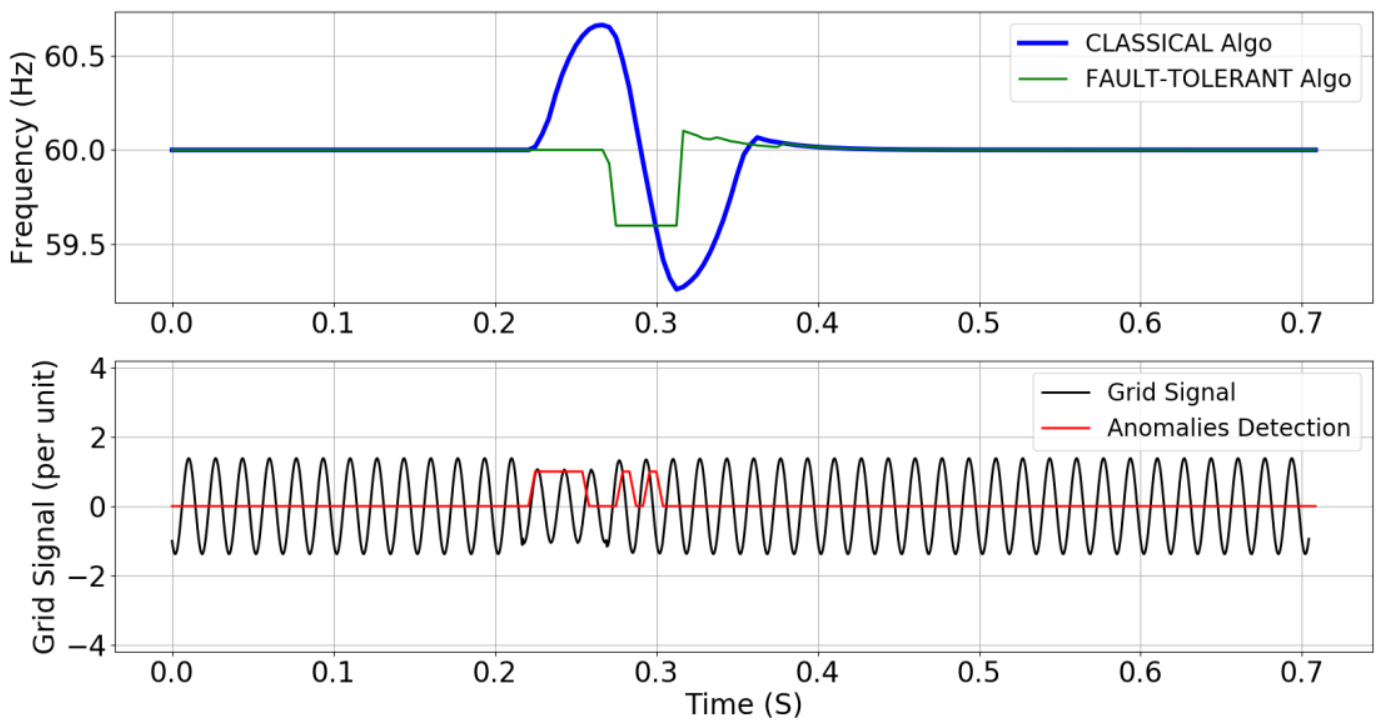

Figure 175 time domain result - case 48 


\section{APPENDIX B. IEEE 13 FEEDER NODE SYSTEM}

Overhead Line Configuration Data:

\begin{tabular}{|c|c|c|c|c|}
\hline Config. & Phasing & Phase & Neutral & Spacing \\
\hline & & ACSR & ACSR & ID \\
\hline 601 & B A C N & $556,50026 / 7$ & $4 / 06 / 1$ & 500 \\
\hline 602 & C A B N & $4 / 06 / 1$ & $4 / 06 / 1$ & 500 \\
\hline 603 & C B N & $1 / 0$ & $1 / 0$ & 505 \\
\hline 604 & A C N & $1 / 0$ & $1 / 0$ & 505 \\
\hline 605 & C N & $1 / 0$ & $1 / 0$ & 510 \\
\hline
\end{tabular}

Underground Line Configuration Data:

\begin{tabular}{|c|c|c|c|c|}
\hline Config. & Phasing & Cable & Neutral & Space ID \\
\hline 606 & A B C N & 250,000 AA, CN & None & 515 \\
\hline 607 & A N & $1 / 0$ AA, TS & $1 / 0 \mathrm{Cu}$ & 520 \\
\hline
\end{tabular}

Line Segment Data:

\begin{tabular}{|c|c|c|c|}
\hline Node A & Node B & Length(ft.) & Config. \\
\hline 632 & 645 & 500 & 603 \\
\hline 632 & 633 & 500 & 602 \\
\hline 633 & 634 & 0 & XFM-1 \\
\hline 645 & 646 & 300 & 603 \\
\hline 650 & 632 & 2000 & 601 \\
\hline 684 & 652 & 800 & 607 \\
\hline 632 & 671 & 2000 & 601 \\
\hline 671 & 684 & 300 & 604 \\
\hline 671 & 680 & 1000 & 601 \\
\hline 671 & 692 & 0 & Switch \\
\hline 684 & 611 & 300 & 605 \\
\hline 692 & 675 & 500 & 606 \\
\hline
\end{tabular}

Transformer Data:

\begin{tabular}{|c|c|c|c|c|c|}
\hline & kVA & kV-high & kV-low & $\begin{array}{c}\text { R } \\
-\%\end{array}$ & X - \% \\
\hline Substation: & 5,000 & 115 - D & 4.16 Gr. Y & 1 & 8 \\
\hline XFM -1 & 500 & 4.16 - Gr.W & 0.48 - Gr.W & 1.1 & 2 \\
\hline
\end{tabular}

Capacitor Data:

\begin{tabular}{|c|c|c|c|}
\hline Node & Ph-A & Ph-B & Ph-C \\
\hline & kVAr & kVAr & kVAr \\
\hline 675 & 200 & 200 & 200 \\
\hline 611 & & & 100 \\
\hline 680 & 200 & 200 & 200 \\
\hline
\end{tabular}

Regulator Data:

\begin{tabular}{|l|l|l|l|}
\hline Regulator ID: & 1 & & \\
\hline
\end{tabular}




\begin{tabular}{|l|c|c|c|}
\hline Line Segment: & $650-632$ & & \\
\hline Location: & 50 & & \\
\hline Phases: & A - B -C & & \\
\hline Connection: & 3-Ph,LG & & \\
\hline Monitoring Phase: & A-B-C & & \\
\hline Bandwidth: & 2.0 volts & & \\
\hline PT Ratio: & 20 & & \\
\hline Primary CT Rating: & 700 & & \\
\hline Compensator Settings: & Ph-A & Ph-B & Ph-C \\
\hline R - Setting: & 3 & 3 & 3 \\
\hline X - Setting: & 9 & 9 & 9 \\
\hline Voltage Level: & 122 & 122 & 122 \\
\hline
\end{tabular}

Spot Load Data:

\begin{tabular}{|c|c|c|c|c|c|c|c|}
\hline Node & Load & Ph-1 & Ph-1 & Ph-2 & Ph-2 & Ph-3 & Ph-3 \\
\hline & Model & $\mathrm{kW}$ & $\mathrm{kVAr}$ & $\mathrm{kW}$ & $\mathrm{kVAr}$ & $\mathrm{kW}$ & $\mathrm{kVAr}$ \\
\hline 634 & Y-PQ & 160 & 110 & 120 & 90 & 120 & 90 \\
\hline 645 & Y-PQ & 0 & 0 & 170 & 125 & 0 & 0 \\
\hline 646 & D-Z & 0 & 0 & 230 & 132 & 0 & 0 \\
\hline 652 & Y-Z & 128 & 86 & 0 & 0 & 0 & 0 \\
\hline 671 & D-PQ & 385 & 220 & 385 & 220 & 385 & 220 \\
\hline 675 & Y-PQ & 485 & 190 & 68 & 60 & 290 & 212 \\
\hline 692 & D-I & 0 & 0 & 0 & 0 & 170 & 151 \\
\hline 611 & Y-I & 0 & 0 & 0 & 0 & 170 & 80 \\
\hline & TOTAL & 1158 & 606 & 973 & 627 & 1135 & 753 \\
\hline
\end{tabular}

Distributed Load Data:

\begin{tabular}{|c|c|c|c|c|c|c|c|c|}
\hline Node A & Node B & Load & Ph-1 & Ph-1 & Ph-2 & Ph-2 & Ph-3 & Ph-3 \\
\hline & & Model & kW & kVAr & kW & kVAr & kW & kVAr \\
\hline 632 & 671 & Y-PQ & 17 & 10 & 66 & 38 & 117 & 68 \\
\hline
\end{tabular}

